Phenotype-based genetic association studies in schizophrenia:

Proof-of-principle for cognition-relevant genes

\author{
PhD THESIS \\ for the award of the degree \\ "Doctor rerum naturalium" (Dr.rer.nat) \\ Division of Mathematics and Natural Sciences \\ of the Georg-August-University Göttingen
}

submitted by

Sabrina Grube geb. Klaus

from Göttingen

Göttingen 2011 


\section{Prof. Dr. Dr. Hannelore Ehrenreich (Reviewer)}

Division of Clinical Neuroscience

Max Planck Institute of Experimental Medicine

Göttingen

\section{Prof. Dr. Nils Brose (Reviewer)}

Department of Molecular Neurobiology

Max Planck Institute of Experimental Medicine

Göttingen

\section{Prof. Dr. Peter Rehling}

Department of Biochemistry II

Georg-August-University

Göttingen 


\section{DECLARATION}

I hereby declare that this thesis has been written independently and with no other sources and aids than quoted.

Sabrina Grube

Göttingen, 09.03.2011 


\section{ACKNOWLEDGEMENTS}

No one can whistle a symphony. It takes a whole orchestra to play it. H.E. Luccock

In this spirit I would like to take the opportunity to thank all the people who contributed in different ways to the completion of my thesis.

First of all I would like to thank Hannelore Ehrenreich for giving me the chance to work under her supervision on this interesting topic in her lab and for the continuous support during the last years. I also thank Nils Brose and Peter Rehling, the additional members of my thesis committee, for their time and enthusiasm.

A special gratitude is dedicated to the members of the AGCT Lab: Fritz Benseler for his unfailing support and advices in every sense and Dayana Schwerdtfeger \& Ivonne Thanhäuser for excellent technical help and nice attempts in- and outside the institute.

I am grateful to Kerstin Reim for helping me a lot with the CPLX2 during the initial phase of my $\mathrm{PhD}$, and for always having time for discussions in between.

Special thanks go to Sergi Papiol for getting me started in the field of genetics, always having time to discuss and being a really helpful good-humored room neighbor. Thanks a lot for the great time we spent together and for your continuous help!

Thanks as well to our collaborators Dörthe Malzahn and Luis Pardo; Dörthe for the statistical support on my projects and fruitful discussions about general statistics and Luis for giving me insights into electrophysiology and conducting the patch clamp measurements. I definitely learned a lot.

Over and above I would like to thank the entire Division of Clinical Neuroscience for providing a supportive work environment and a lot of fun! Especially Heidi, Katja and Fungi for the nice database work and many discussions. Kostik, Ahmed and Bartosz for the behavioral studies. Swetlana and Kathrin for the outstanding technical help and many encouraging conversations. Nora, Lilli and our former colleague Derya for permanent discussions about experiments and other pivotal topics, nice breaks and the good atmosphere in the lab.

My deepest and warmest gratitude, however, go to my family and to my friends who always stood by my side. Thanks for your unfailing support, your understanding, and encouragement even in times I was hardly there. Special thanks go to my lovely husband, for taking care and listen in all circumstances and supporting me wherever he could.

Thank you all so much for your support! 


\section{TABLE OF CONTENTS}

1. GENERAL INTRODUCTION...................................................................................................... 1

1.1 Schizophrenia 1

1.2 Genetics of Schizophrenia 2

1.3 GRAS 3

1.4 Aim of this work 3

2. CPLX2 POLYMORPHISMS MODIFY COGNITIVE PERFORMANCE IN SCHIZOPHRENIA............. 4

2.1 Overview of Project I 4

2.2 Original Publication 6

3. A CAG REPEAT POLYMORPHISM OF KCNN3 PREDICTS SK3 CHANNEL FUNCTION AND

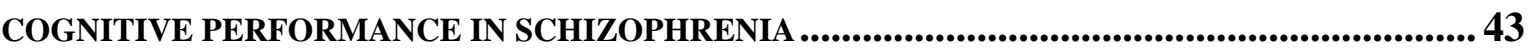

$3.1 \quad$ Overview of Project II 43

3.2 Original Publication 46

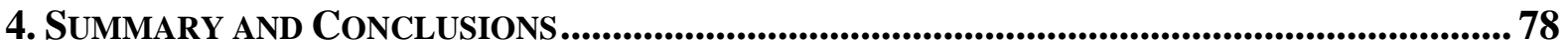

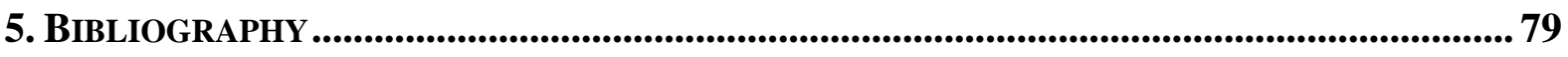

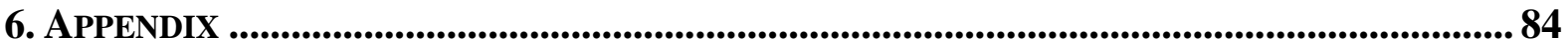

$\begin{array}{lll}6.1 & \text { Curriculum Vitae } & 84\end{array}$

6.2 Accepted co-author publications 85 


\section{GENERAL INTRODUCTION}

\subsection{SCHIZOPHRENIA}

Schizophrenia is the name for a multifaceted psychiatric disease that affects around $1 \%$ of the population worldwide (Jablensky et al, 1992). The diagnosis schizophrenia is made according to the leading classification systems DSM-IV (AmericanPsychiatricAssociation, 2000) and ICD-10 (WorldHealthOrganization, 1992) and is exclusively based on the clinical appearance of the patients. Two main types of symptoms reflect the psychotic disturbances: positive symptoms including hallucinations or delusions and negative symptoms as apathy, lack of emotion or social withdrawal. Additional cognitive deficits are accompanying the disease. These and other mild behavioral abnormalities may already be present in childhood but the general characteristics of the disease appear in young adulthood (Erlenmeyer-Kimling, 2001). Because of the early onset and the pervasiveness of associated deficits, the disease places a substantial burden on the patients, their families and society. It is among the top ten leading causes of disease-related disability in the world (Murray and Lopez, 1996); alone the costs for schizophrenia in the US in 2002 were estimated to be $\$ 62.7$ billion (McEvoy, 2007).

Though there is no cure for schizophrenia, since the mid-1950s a variety of treatment options are available. The main treatment is antipsychotic medication that helps to normalize the biochemical imbalance that is thought to contribute to schizophrenia (Stephan et al, 2006). These drugs primarily work by suppressing dopamine activity and are effective in treating the positive symptoms (Freedman, 2003). Unfortunately, this treatment has only limited efficacy on negative symptoms and cognitive deficits (Carpenter, 2004; Keefe et al, 2007), which appear to have the main impact on the quality of life of the patients (Hyman and Fenton, 2003).

The underlying biological mechanisms for this complex brain disease with neurodevelopmental origin (Rapoport et al, 2005) are far from clear. Evidence suggests a complex interplay between a genetic predisposition and environmental conditions during preand postnatal development. In a recent review by Jim van Os and colleagues (van Os et al, 2010) developmental trauma, a minority group position, growing up in an urban environment and cannabis use have been reported to have a predisposing impact on the developing brain. Most probably complex gene $\mathrm{x}$ environment interactions lead finally to a syndrome that we call schizophrenia in our days. 
Although cognition is not a component of the DSM-IV/ICD-10 criteria, it is undisputable that cognitive deficits are the core of the disease (Elvevag and Goldberg, 2000). They have been already considered in the earliest descriptions of schizophrenia by Kraepelin as 'dementia praecox' in the late $19^{\text {th }}$ century (Kraepelin, 1919). The majority (Palmer et al, 1997), if not all (Keefe et al, 2005) schizophrenic patients suffer from these neuropsychological abnormalities, mainly seen in the domains of attention, memory and executive functions (Kuperberg and Heckers, 2000). The biological brain correlate is most probably a widespread dysfunction within a network of brain areas involving the frontal and temporal cortex, hippocampus and subcortical regions (Kuperberg and Heckers, 2000). Interestingly these deficits are mainly independent of the disease and treatment state (Gold et al, 1999) and are visible even before the onset of the first psychotic episode (Reichenberg et al, 2002). Furthermore healthy twins and relatives from affected people show slight abnormalities when compared to healthy controls (Cannon et al, 2000; Snitz et al, 2006), leading to the question whether there is shared genetic liability (Toulopoulou et al, 2010). Moreover cognitive deficits are among the best predictors for the disease outcome and quality of life (Addington and Addington, 1999; Green, 1996). Taken together, impaired cognition is one of the most prominent phenotypes in schizophrenia research (e.g. Burdick et al, 2009; Gottesman and Gould, 2003).

\subsection{GENETICS OF SCHIZOPHRENIA}

There is no doubt a strong genetic component to schizophrenia, which has been proven by twin, family and adoption studies. Recent estimates for the heritability are $\sim 80 \%$ (Sullivan et $a l$, 2003) which leaves space for a significant proportion of environmental influences. The risk genes that are discussed are multivariate; in addition, the suggested candidate genes have not been consistently replicated across or even within populations (e.g. Sanders et al, 2008). The recent genome-wide association (GWAS) and copy number variation (CNV) studies have provided important evidence suggesting a role of both, common and rare variants in schizophrenia genesis (for review see Tiwari et al, 2010). The most consistent observations among these studies are the association of genetic markers in the major histocompatibility complex (MHC) on chromosome 6p22.1 and an excess of rare variants in patients with schizophrenia. Despite the increasing knowledge in genetics, we have not learned a lot about biological correlates so far. The understanding of the underlying biological mechanisms would be a major step forward to developing novel and maybe even individualized treatments for this heterogeneous group of patients. 


\subsection{GRAS}

To get a better understanding of the biological grounds of schizophrenia and to find out how common genetic variants contribute to the phenotype, the Göttingen Research Association of Schizophrenia was founded in 2005 (Ribbe et al, 2010). Until 2008, an invariant team of physicians and psychologists traveled throughout Germany and examined more than 1000 patients diagnosed with schizophrenia according to DSM-IV. The acquired dataset includes biographic and family information, disease history, treatments, environmental risk factors, comorbidities, and additionally results of neuropsychological, psychopathological, and neurological examinations. With more than 3000 data points / patient, we have a very comprehensive unique phenotypical database that is ideally suited for phenotype-based genetic association studies (PGAS).

\subsection{AIM OF THIS WORK}

Starting with my project in 2008, I had the opportunity to conduct the first genetic analyses within our GRAS database. The goal was to start where other groups ended. The idea is not only to do a case-control study comparing the genotypes in our schizophrenic population with healthy controls based on endpoint diagnosis but perform comprehensive, hypothesis driven phenotype-based genetic association studies to see how specific common genetic variants contribute to the phenotype. Moreover we tried to find out how these variants influence the biological system e.g. the expression of the respective RNA or protein or the function. The two already concluded studies shown here, focus on genetic modifiers of cognition and are the first of this kind in the field. 


\section{CPLX2 POLYMORPHISMS MODIFY COGNITIVE PERFORMANCE IN SCHIZOPHRENIA}

\subsection{Overview of Project I}

Dysfunction and disruption of functional synaptic connectivity have been proposed to play a key role in schizophrenia (Owen et al, 2005). Having in mind that neurotransmitter release is the fastest and most tightly regulated fusion event, a putative molecular mechanism might involve abnormalities of proteins participating in the presynaptic secretory machinery (Sawa and Snyder, 2002), among them are complexins (CPLXs).

CPLXs are a family of four small (134-160 aa) highly charged proteins that are highly conserved among mammals (McMahon et al, 1995). They are essential for the regulation of synaptic transmitter release by controlling assembly and stability of exocytotic SNARE complexes and thereby influence synaptic signaling, synaptic plasticity, and neuronal network function (for review see Brose, 2008a).

While CPLX2 is also detectable in non-neuronal tissue, CPLX1 is specifically expressed in the central nervous system (McMahon et al, 1995). In the brain, the two isoforms show an overlapping expression pattern; many neurons express both proteins, albeit at different levels (Reim et al, 2001; Reim et al, 2005). CPLX3/4 are predominantly expressed at ribbon synapses in the retina (Reim et al, 2005).

Genetic removal of complexins in mammalian cells leads to a strong reduction in evoked neurotransmitter release (Huntwork and Littleton, 2007; Reim et al, 2001). That is why altered expression of complexins is thought to be involved in several diseases. In post mortem studies of e.g. Huntington's (Morton et al, 2001), Parkinson's (Basso et al, 2004), Alzheimer's (Tannenberg et al, 2006) diseases and schizophrenia (e.g. Eastwood and Harrison, 2005), altered levels of complexins were detected in the brain (for review see Brose, 2008b).

Especially for schizophrenia there are a number of reports available describing mRNA and protein changes for CPLX1 and CPLX2 in brain regions highly affected in this disease (e.g. dorsolateral prefrontal cortex, superior temporal cortex, and certain regions of the hippocampus). Overall the results point to a decreased mRNA expression of complexins in schizophrenia (Brose, 2008b). Along this line, a systematic transcriptome analysis showed 
that many mRNAs coding for synaptic proteins are decreased in schizophrenic patients (Mirnics et al, 2001).

These results strengthen the hypothesis for schizophrenia as a disease of the synapse, but leaving the question whether complexins have a causal role in the etiology or they just contribute to the corresponding symptoms unanswered. CPLX1 and 2 expression changes were indeed found to be associated with cognitive deficits (Sawada et al, 2005). Behavioral consequences have been studied as well in different genetic mouse models. Cplx1 null mutant mice show severe ataxia, but do not have a clear cognitive phenotype (Drew et al, 2007). Cplx2 null mutants behave substantially normal, show only slight motor abnormalities and disputable results regarding cognition (Glynn et al, 2003; Glynn et al, 2007; Yamauchi et al, 2005). Genetic association studies in human population have been contradictory (Kishi et al, 2006; Lee et al, 2005) but showing rather no association with schizophrenia.

Based on these findings we analyzed genetic variability in the CPLX2 gene in the GRAS cohort. We hypothesized that the common variants found are not associated with schizophrenia itself but rather showing a modulatory influence on the cognitive phenotype. In addition we aimed to understand the underlying biological reasons, (i) modeling similar cognitive behavior in Cplx2 null mutant mice and (ii) conducting in vitro expression studies.

Taken the results together we can conclude that CPLX2 has a modifier role in cognition in situations of additional challenge, which holds true for both mouse and man. Interestingly, these findings are due to a lower expression of the protein, in humans modulated by a common genetic variant in the 3'UTR of the gene interacting with a miRNA. 


\subsection{Original Publication}

Begemann $\mathrm{M}^{*}$, Grube S*, Papiol S, Malzahn D, Krampe H, Ribbe K, Friedrichs H, Radyushkin KA, El-Kordi A, Benseler F, Hannke K, Sperling S, Schwerdtfeger D, Thanhauser I, Gerchen MF, Ghorbani M, Gutwinski S, Hilmes C, Leppert R, Ronnenberg A, Sowislo J, Stawicki S, Stodtke M, Szuszies C, Reim K, Riggert J, Eckstein F, Falkai P, Bickeboller H, Nave KA, Brose N, Ehrenreich H (2010). Modification of Cognitive Performance in Schizophrenia by Complexin 2 Gene Polymorphisms. Arch Gen Psychiatry 67(9), 879-888.

* Indicates equal contribution for the publication

Personal contribution:

I carried out all experimental work except for mouse behavior: sequencing, selecting SNPs and genotyping, computational sequence analysis, cloning and luciferase assays and the expression study in PBMCs. For these parts of the paper I was responsible for the design of the experiments and conducted the statistical analysis. Additionally, I was involved in the interpretation of all results, review of the literature, and in manuscript preparation, writing and revision. 


\title{
Modification of Cognitive Performance in Schizophrenia by Complexin 2 Gene Polymorphisms
}

\author{
Martin Begemann, MD*; Sabrina Grube, MSc*; Sergi Papiol, PhD; Dörthe Malzahn, PhD; Henning Krampe, PhD; \\ Katja Ribbe, MSc; Heidi Friedrichs, MSc; Konstantin A. Radyushkin, PhD; Ahmed El-Kordi, MSc; Fritz Benseler, BA; \\ Kathrin Hannke, BA; Swetlana Sperling, BA; Dayana Schwerdtfeger, BA; Ivonne Thanhäuser, BA; \\ Martin F. Gerchen, BSc; Mohammad Ghorbani, MD; Stefan Gutwinski, MD; Constanze Hilmes, MD; \\ Richard Leppert, MD; Anja Ronnenberg, BA; Julia Sowislo, BSc; Sabina Stawicki, PhD; Maren Stödtke, MD; \\ Christoph Szuszies, MD; Kerstin Reim, PhD; Joachim Riggert, MD; Fritz Eckstein, PhD; Peter Falkai, MD; \\ Heike Bickeböller, PhD; Klaus-Armin Nave, PhD; Nils Brose, PhD; Hannelore Ehrenreich, MD, DVM
}

\begin{abstract}
Context: Schizophrenia is the collective term for a heterogeneous group of mental disorders with a still obscure biological basis. In particular, the specific contribution of risk or candidate gene variants to the complex schizophrenic phenotype is largely unknown.
\end{abstract}

Objective: To prepare the ground for a novel "phenomics" approach, a unique schizophrenia patient database was established by GRAS (Göttingen Research Association for Schizophrenia), designed to allow association of genetic information with quantifiable phenotypes. Because synaptic dysfunction plays a key role in schizophrenia, the complexin 2 gene (CPLX2) was examined in the first phenotype-based genetic association study (PGAS) of GRAS.

Design: Subsequent to a classic case-control approach, we analyzed the contribution of CPLX2 polymorphisms to discrete cognitive domains within the schizophrenic population. To gain mechanistic insight into how certain CPLX2 variants influence gene expression and function, peripheral blood mononuclear cells of patients, $C p l x-$ null mutant mice, and transfected cells were investigated.

Setting: Coordinating research center (Max Planck Institute of Experimental Medicine) and 23 collaborating psychiatric centers all over Germany.

Participants: One thousand seventy-one patients with schizophrenia (DSM-IV) examined by an invariant in- vestigator team, resulting in the GRAS database with more than 3000 phenotypic data points per patient, and 1079 healthy control subjects of comparable ethnicity.

Main Oułcome Measure: Cognitive performance including executive functioning, reasoning, and verbal learning/memory.

Results: Six single-nucleotide polymorphisms, distributed over the whole CPLX2 gene, were found to be highly associated with current cognition of schizophrenic subjects but only marginally with premorbid intelligence. Correspondingly, in Cplx2-null mutant mice, prominent cognitive loss of function was obtained only in combination with a minor brain lesion applied during puberty, modeling a clinically relevant environmental risk ("second hit") for schizophrenia. In the human CPLX2 gene, 1 of the identified 6 cognition-relevant single-nucleotide polymorphisms, rs 3822674 in the $3^{\prime}$ untranslated region, was detected to influence microRNA-498 binding and gene expression. The same marker was associated with differential expression of CPLX2 in peripheral blood mononuclear cells.

Conclusions: The PGAS allows identification of markerassociated clinical/biological traits. Current cognitive performance in schizophrenic patients is modified by CPLX2 variants modulating posttranscriptional gene expression.

Arch Gen Psychiatry. 2010;67(9):879-888
Author Affiliations are listed at the end of this article.

*Indicates co-primary authorship.

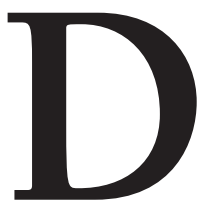

ETAILED KNOWLEDGE OF the complex gene-environment interactions underlying the etiology and pathogenesis of the schizophrenias $^{1}$ is required to understand the causes of the disease and to establish causal therapies. However, the search for genetic markers of vulnerability for schizophrenia has been hampered by extensive interfering effects of patient heterogeneity and environmental fac- tors. ${ }^{2}$ Indeed, many of the numerous genetic association studies have yielded poorly reproducible data, likely because corresponding patient cohorts were too small and/or too heterogeneous with regard to clinical characteristics. One way to circumvent the problems that are posed by the lack of exact phenotyping in the available schizophrenia samples, and thus confound genetic studies, is to analyze large patient cohorts. Three recent studies of this type $e^{3-5}$ showed that several com- 
mon genetic variations contribute to the risk of schizophrenia, but the functional consequences of these variations are still completely unknown.

In the present study, we opted for an alternative approach to study genetic causes of the schizophrenic phenotype: a phenotype-based genetic association study (PGAS). This approach is different from and complementary to the genome-wide association studies on schizophrenia as a disease. With the PGAS, we are not looking for major disease genes in schizophrenia because such genes may not exist. Rather than searching for schizophrenia genes, we seek to learn more about the contribution of genetic variants of certain candidate genes to the schizophrenic phenotype. Obviously, traits of interest in schizophrenia can never be explained only by a single modifier gene. However, a particular gene may codetermine (with other trait-relevant genes) the outcome of an individual with schizophrenia.

Based on the assumption that valuable information about relevant genetic disease mechanisms can be obtained by association studies on patient cohorts of at least $1000 \mathrm{pa}-$ tients, if performed on very detailed clinical data sets and quantifiable biological readouts of schizophrenia rather than the end point diagnosis in comparison with healthy controls, we generated a new schizophrenia patient database, the GRAS (Göttingen Research Association for Schizophrenia) data collection. For this purpose, 1071 patients with schizophrenia were recruited between July 21, 2005, and July 7, 2008, by one and the same team of traveling investigators in a cross-sectional field study, consisting of 23 German psychiatric hospitals (listed in the supplementary Appendix; http://www.archgenpsychiatry.com). The corresponding data set includes biographical and family information, disease history, environmental risk factors, comorbidities, treatments, and the results of cross-sectional psychopathological, neuropsychological, and neurological examinations. With more than 3000 data points per subject, this unique database of living patients, who are accessible for follow-up studies, provides a comprehensive and standardized phenotype characterization of as yet unprecedented detail.

Neurocognitive impairments, including deficits in executive functions, attention, and memory, are core symptoms of schizophrenia and the main cause of diseaserelated disability. ${ }^{6}$ With regard to these and other schizophrenia symptoms, synaptic dysfunctions and disruptions of functional synaptic connectivity have been proposed to play a key role, and some authors even call schizophrenia a disease of the synapse. ${ }^{7-9}$ Numerous reports have implicated genes encoding synaptic proteins in the etiology of schizophrenia. ${ }^{10,11}$ Postmortem studies on the brain tissue of schizophrenic patients have consistently observed alterations in the expression of several synaptic proteins. In this context, the complexin (CPLX) family of presynaptic regulatory proteins is particularly interesting. ${ }^{12-18}$ This family consists of 4 members (CPLX1-CPLX4), of which only Cplx1 and Cplx2 are strongly expressed in rodent forebrain. ${ }^{19}$ They play an essential role in the regulation of synaptic transmitter release by controlling assembly and stability of exocytotic soluble $\mathrm{N}$-ethylmaleimide-sensitive factor attachment protein receptor (SNARE) complexes and thereby influence synaptic signaling, ${ }^{20,21}$ synaptic plasticity, ${ }^{22,23}$ and neuronal network function. ${ }^{24}$

Whereas previous genetic association studies exploring CPLX1 (OMIM 605032) or CPLX2 (OMIM 605033) as classic genetic risk factors for schizophrenia have yielded inconclusive results, ${ }^{25,26}$ interesting findings were described regarding CPLX1 and CPLX2 messenger RNA (mRNA) and protein analysis in postmortem brain tissue. Although no systematic overall analysis of CPLX1 and CPLX2 expression patterns in normal human brain has been published, a number of reports are available on CPLX mRNA and protein changes in discrete brain areas of schizophrenic patients and control subjects, providing evidence of a decreased CPLX2 mRNA expression in regions of high relevance for schizophrenia (eg, dorsolateral prefrontal cortex, superior temporal cortex, and certain regions of the hippocampus). A correspondence to decreased CPLX2 protein levels in these regions is difficult to establish because the literature is scarce..$^{27}$

In addition to schizophrenia, altered CPLX/CPLX levels were measured in different brain areas of patients with diverse other neuropsychiatric disorders and implicated in the disease process, perhaps as determinants of a final common pathological pathway. Among these neuropsychiatric disorders are Huntington disease, ${ }^{28,29}$ Alzheimer disease, ${ }^{30}$ and bipolar disorder, ${ }^{14,17}$ all of them characterized, among others, by cognitive impairments. Interestingly, CPLX1 and CPLX2 expression changes in the hippocampus were found to be associated with cognitive deficits in schizophrenia, ${ }^{31}$ still leaving unanswered whether the altered tissue levels reflect the cause or the consequence of disease-related cognitive dysfunction.

In this regard, behavioral consequences observed in null mutant mice have to be considered. Although Cplx1null mutant mice show severe ataxia, they have no clear cognitive phenotype and only subtle alterations in social behavior. ${ }^{32}$ The Cplx2-null mutant mice have an even milder phenotype with slight motor abnormalities and equivocal results regarding cognition under unchallenged conditions. ${ }^{33-35}$ However, when maternal deprivation stress presents a second hit, cognitive dysfunction and decreased induction of hippocampal long-term potentiation in Cplx2-null mutant mice become evident. ${ }^{34} \mathrm{~A}$ similar second hit effect may be involved in the disease-related function of another presynaptic protein, 25-kDa synaptosome-associated protein (SNAP-25). ${ }^{36}$

Taking all these observations together, we hypothesized that common genetic variants of CPLX2 may play an important role as modifiers of cognition in situations of additional challenge. Such a second hit to the brain, which ultimately leads to the disease, may cause a schizophrenic phenotype of variable severity depending on the genetic variant present. Searching for mechanistic insight, we hypothesized that the respective variants, if located in the noncoding region of the gene, may influence quantitative gene expression, for example, by modulating binding of microRNAs (miRs), which are increasingly recognized as significant contributors to the adaptive fine-tuning of synaptic functions in the brain. ${ }^{37,38}$ To lend further support to our second hit concept, we investigated the effect of a mild peripubertal neurotrauma on cognition in Cplx2-null mutant mice.

\footnotetext{
$\overline{\text { ARCH GEN PSYCHIATRY/VOL } 67 \text { (NO. 9), SEP } 2010 \text { WWW.ARCHGENPSYCHIATRY.COM }}$ 
METHODS

A more comprehensive description of the patient population and all methods and materials applied is provided in the supplementary Methods section (see also eTable 1).

\section{SCHIZOPHRENIC SUBJECTS}

The GRAS data collection was approved by the Ethics Committee of the Georg-August-University (master committee) and the local internal review boards of the collaborating centers. The project complied with the Helsinki declaration. ${ }^{39}$ Patients fulfilling DSM-IV criteria for schizophrenia ${ }^{40,41}$ (all types, eg, paranoid, disorganized, catatonic, and undifferentiated ${ }^{42}$ proven or suspected) or schizoaffective disorder were included $(\mathrm{N}=1071)$, regardless of the stage of the disorder (acute, chronic, residual, or remitted). A total of 792 patients (73.9\%) were diagnosed as having schizophrenia; 159 (14.8\%), schizoaffective disorder; and 120 (11.2\%), suspected schizophrenic psychosis (other psychotic disorder or yet to be confirmed). The mean (SD) age was 39.6 (12.8) years, with a range from 18 to 83 years. Seven hundred fourteen $(66.7 \%)$ were men (age, 37.6 [12.0] years) and 357 (33.3\%) were women (age, 43.7 [13.3] years). Subjects (all older than 18 years) and, if applicable, their legal representatives, gave written informed consent.

Patients were recruited in the 23 German psychiatric hospitals listed in the supplementary Appendix, and almost all of them were of European Caucasian descent (Caucasian, 95.3\%; other, $1.6 \%$; unknown, 3.1\%). European Caucasians are a genetically homogeneous group with low average levels of genetic differentiation when compared with other human populations (no strong influence on association results to be expected). ${ }^{43-45}$ Specifically, the German population is very homogeneous, with a low genetic differentiation along a northsouth gradient within Germany. In fact, population substructure within Germany is too low to be detectable without prior information on subpopulation membership. ${ }^{46}$

\section{CONTROL SUBJECTS}

The control subjects were voluntary blood donors recruited by the Department of Transfusion Medicine at the Georg-AugustUniversity according to national guidelines for blood donation. As such, they widely fulfill health criteria, ensured by a broad predonation screening process containing standardized questionnaires, interviews, hemoglobin levels, and blood pressure, pulse, and body temperature determinations. Of the 1079 controls, 635 (58.9\%) were men and 444 (41.1\%) were women. The mean (SD) age was 34.7 (12.3) years, with a range from 18 to 69 years. Comparable to the patient population, almost all controls were of European Caucasian descent (Caucasian, $97.8 \%$; other, 2.0\%; unknown, $0.2 \%$ ). All donors gave written informed consent.

\section{PHENOTYPING}

Comprehensive interviews and testing were performed by the same traveling team of trained examiners (psychiatrists and psychologists) using the GRAS Manual described in the supplementary Methods. Briefly, structured interviews were conducted to explore biographical and family information, level of education, quality-of-life indicators, disease history, and exposure to prenatal, perinatal, and/or postnatal environmental risk factors. Likewise, the psychopathological profile, psychiatric comorbidities, and current/former treatments were assessed. Psychometric rating, neuropsychological tests, and neurological examinations were also performed.

\section{GENOTYPING}

Standard methods were used for DNA extraction from peripheral blood cells (Genomed GmbH, Löhne, Germany). Sequencing was performed using the dideoxy chain termination method (BigDye Terminator version 3.1 cycle sequencing kit on a 3730XL DNA analyzer; Applied Biosystems, Foster City, California). Genotyping was performed with simple probes (TIB Molbiol, Berlin, Germany) on a real-time polymerase chain reaction instrument (LightCycler 480; Roche Diagnostics GmbH, Mannheim, Germany).

\section{EXPRESSION ANALYSIS}

Expression analysis was conducted in Neuro-2a (N2a) cells (LGC Standards GmbH, Wesel, Germany) with the dualluciferase reporter system (Promega, Mannheim, Germany). Briefly, the first 274 base pairs (bp) of the 3' untranslated region (3'UTR) of CPLX2, containing single-nucleotide polymorphism (SNP) rs3822674 with a C or a T allele, were amplified from respective human samples and cloned into Renilla luciferase vector phRL-SV40 (Promega). The N2a cells were plated in 96-well plates, cultured for 16 to 18 hours, and transfected using a commercially available reagent (Lipofectamine 2000; Invitrogen, Karlsruhe, Germany). A total of 1 ng of phRLSV40 ( $\mathrm{T}$ or $\mathrm{C}$ construct or the vector without the insert) and 1 pg of pCMV-FFluc control vector (Promega) were cotransfected with/without 1 pmol of hsa-miR-498 (Ambion, Foster City, California). The dual-luciferase reporter assay was performed 24 hours after transfection according to the manufacturer's protocol (Promega). Measurements were conducted with a commercially available microplate reader (Mitras LB 940; Berthold Technologies GmbH, Regensdorf, Switzerland). Renilla values were divided by the corresponding firefly readings producing values expressed as relative luciferase units.

\section{ISOLATION OF PERIPHERAL BLOOD MONONUCLEAR CELLS AND QUANTITATIVE REAL-TIME REVERSE TRANSCRIPTASE-POLYMERASE CHAIN REACTION}

Blood was collected in citrate phosphate dextrose adenine tubes from schizophrenic patients with different genotypes (CC, CT, and TT) at SNP rs3822674 in the 3'UTR. Six patients had the CC genotype (mean [SD] age, 48.5 [5.3] years); of these, 3 were men (age, 52.3 [4.7] years) and 3 were women; (age, 44.6 [10.1] years). Four had the CT genotype (age, 41.8 [3.4] years), and all 4 were men. Six had the TT genotype (age, 47.5 [5.8] years); of these, 5 were men (age, 45.6 [6.7] years) and 1 was a woman (age, 57 years). Peripheral blood mononuclear cells (PBMCs) were isolated applying a standard isolation procedure (FicollPaque Plus; GE Healthcare, München, Germany). The RNA was prepared using a commercially available kit (miRNeasy Mini Kit; Qiagen GmbH, Hilden, Germany). The RNA samples were used to synthesize complementary DNAs (SuperScriptIII; Invitrogen). The quantitative real-time polymerase chain reaction analysis was performed using the fluorescent dye SYBR Green (LightCycler 480; Roche Diagnostics GmbH). The cycle threshold values were standardized to the cycle threshold values of glyceraldehyde-3-phosphate dehydrogenase. Primers are listed in the supplementary "Methods" section.

\section{ANIMAL BEHAVIOR}

Male Cplx2-null mutant mice vs wild-type littermates with or without juvenile parietal cortical cryolesion ${ }^{47}$ underwent behavioral testing, including the Morris water maze, at 10 months of age.

\footnotetext{
$\overline{\text { ARCH GEN PSYCHIATRY/VOL } 67 \text { (NO. 9), SEP } 2010 \text { WWW.ARCHGENPSYCHIATRY.COM }}$ 
Table. Neurocognitive Performance Associated With CPLX2 SNPs a

\begin{tabular}{|c|c|c|c|c|c|c|c|c|c|c|c|c|c|}
\hline \multirow[b]{2}{*}{ Phenotype } & \multicolumn{11}{|c|}{ SNP } & \multirow[b]{2}{*}{$K^{d}$} & \multirow{2}{*}{$\begin{array}{c}\text { Permutation } \\
\text { Test } \\
P \text { Value } \\
(95 \% \text { CI) }\end{array}$} \\
\hline & rs6868608 & rs $2443541^{b}$ & rs2243404 & rs4242187 & rs $10072860^{b}$ & rs4868539 & rs $1366116^{b}$ & rs3892909b & rs3822674 ${ }^{\mathrm{b}, \mathrm{c}}$ & rs56934064 & $\begin{array}{c}+251 \\
\text { Ex6 }\end{array}$ & & \\
\hline \multicolumn{14}{|c|}{ Target (Modifier) Variables } \\
\hline \multicolumn{14}{|l|}{ Combined $^{f}$} \\
\hline$F(P)$ values & $\begin{array}{l}0.310 \\
(.733)\end{array}$ & $\begin{array}{l}2.827 \\
(.059)\end{array}$ & $\begin{array}{l}3.705 \\
(.025)\end{array}$ & $\begin{array}{l}0.679 \\
(.507)\end{array}$ & $\begin{array}{l}2.254 \\
(.105)\end{array}$ & $\begin{array}{l}0.868 \\
(.420)\end{array}$ & $\begin{array}{l}4.288 \\
(.014)\end{array}$ & $\begin{array}{l}5.073 \\
(.006)\end{array}$ & $\begin{array}{l}3.288 \\
(.037)\end{array}$ & $\begin{array}{l}0.423 \\
(.655)\end{array}$ & $\begin{array}{l}0.517 \\
(.596)\end{array}$ & 4 & $\begin{array}{c}.020 \\
(.017-.023)\end{array}$ \\
\hline \multirow{2}{*}{\multicolumn{14}{|c|}{$\begin{array}{l}\text { Individualg } \\
\text { Exec }\end{array}$}} \\
\hline \multicolumn{4}{|l|}{ Exec } & & & & & & & & & & \\
\hline$F(P)$ values & $\begin{array}{l}1.554 \\
(.212)\end{array}$ & $\begin{array}{l}4.238 \\
(.015)\end{array}$ & $\begin{array}{l}3.621 \\
(.027)\end{array}$ & $\begin{array}{l}2.000 \\
(.136)\end{array}$ & $\begin{array}{l}3.690 \\
(.025)\end{array}$ & $\begin{array}{l}0.673 \\
(.510)\end{array}$ & $\begin{array}{l}1.753 \\
(.174)\end{array}$ & $\begin{array}{l}1.808 \\
(.165)\end{array}$ & $\begin{array}{l}1.043 \\
(.353)\end{array}$ & $\begin{array}{l}0.459 \\
(.632)\end{array}$ & $\begin{array}{l}0.279 \\
(.759)\end{array}$ & & \\
\hline $\begin{array}{l}\text { Reasoning } \\
\qquad F(P) \text { values }\end{array}$ & $\begin{array}{l}0.670 \\
(.512)\end{array}$ & $\begin{array}{l}1.562 \\
(.210)\end{array}$ & $\begin{array}{l}1.722 \\
(.179)\end{array}$ & $\begin{array}{l}1.361 \\
(.257)\end{array}$ & $\begin{array}{l}2.567 \\
(.077)\end{array}$ & $\begin{array}{l}1.357 \\
(.258)\end{array}$ & $\begin{array}{l}5.327 \\
(.005)\end{array}$ & $\begin{array}{l}5.434 \\
(.004)\end{array}$ & $\begin{array}{l}4.409 \\
(.012)\end{array}$ & $\begin{array}{l}0.374 \\
(.688)\end{array}$ & $\begin{array}{l}0.366 \\
(.694)\end{array}$ & 9 & $\begin{array}{c}\text { Block } \\
.012 \\
(.010-.014)\end{array}$ \\
\hline$F(P)$ values & $\begin{array}{l}1.186 \\
(.306)\end{array}$ & $\begin{array}{l}3.321 \\
(.037)\end{array}$ & $\begin{array}{l}4.437 \\
(.012)\end{array}$ & $\begin{array}{l}0.520 \\
(.594)\end{array}$ & $\begin{array}{l}0.202 \\
(.817)\end{array}$ & $\begin{array}{l}0.310 \\
(.733)\end{array}$ & $\begin{array}{l}2.218 \\
(.109)\end{array}$ & $\begin{array}{l}3.239 \\
(.040)\end{array}$ & $\begin{array}{l}1.294 \\
(.275)\end{array}$ & $\begin{array}{l}1.012 \\
(.364)\end{array}$ & $\begin{array}{l}0.603 \\
(.548)\end{array}$ & & \\
\hline \multicolumn{14}{|c|}{ Constitutive Variable } \\
\hline \multicolumn{14}{|l|}{$\begin{array}{l}\text { Premorbid } \\
\text { intelligence }\end{array}$} \\
\hline$F(P)$ values & $\begin{array}{l}1.500 \\
(.224)\end{array}$ & $\begin{array}{l}1.159 \\
(.314)\end{array}$ & $\begin{array}{l}2.185 \\
(.113)\end{array}$ & $\begin{array}{l}0.494 \\
(.610)\end{array}$ & $\begin{array}{l}2.107 \\
(.122)\end{array}$ & $\begin{array}{l}0.076 \\
(.927)\end{array}$ & $\begin{array}{l}2.309 \\
(.100)\end{array}$ & $\begin{array}{l}4.511 \\
(.011)\end{array}$ & $\begin{array}{l}0.754 \\
(.471)\end{array}$ & $\begin{array}{l}0.867 \\
(.420)\end{array}$ & $\begin{array}{l}0.481 \\
(.618)\end{array}$ & 1 & $\begin{array}{c}.357 \\
(.348-.366)\end{array}$ \\
\hline \multicolumn{14}{|c|}{ Control Variable } \\
\hline GAF & & & & & & & & & & & & & \\
\hline$F(P)$ values & $\begin{array}{l}0.442 \\
(.643)\end{array}$ & $\begin{array}{l}1.875 \\
(.154)\end{array}$ & $\begin{array}{l}1.579 \\
(.207)\end{array}$ & $\begin{array}{l}0.404 \\
(.668)\end{array}$ & $\begin{array}{l}0.131 \\
(.877)\end{array}$ & $\begin{array}{l}1.059 \\
(.347)\end{array}$ & $\begin{array}{l}1.413 \\
(.244)\end{array}$ & $\begin{array}{l}0.143 \\
(.867)\end{array}$ & $\begin{array}{l}1.068 \\
(.344)\end{array}$ & $\begin{array}{l}0.633 \\
(.531)\end{array}$ & $\begin{array}{l}0.803 \\
(.448)\end{array}$ & 0 & $\begin{array}{c}1.000 \\
(1.000-1.000)\end{array}$ \\
\hline
\end{tabular}

Abbreviations: $\mathrm{Cl}$, confidence interval; Exec, executive functioning; GAF, Global Assessment of Functioning Scale; L/M, learning/memory; SNP, single-nucleotide polymorphism.

${ }^{\text {a }}$ Single locus association analyses of phenotypes with CPLX2, adjusted for sex and age and, in the case of premorbid intelligence, additionally for nonnative German speakers with language problems ( $8.6 \%$ of the total sample; $1.9 \%$ had to be taken out completely owing to severe language difficulties [described in the supplementary "Methods" section]). Significant test statistics ( $F$ values, $2 d f)$ and $P$ values are displayed in boldface type $(P<.05)$.

${ }^{b}$ Markers that underwent haplotypic analysis.

'The SNP in the $3^{\prime}$ untranslated region, affecting the miR-498 binding site.

${ }^{\mathrm{d}}$ The observed number of $P$ values less than .05 on all tests for the multivariate phenotype, for the 3 univariate phenotypes, for the premorbid intelligence, as well as for the control variable (GAF).

e Multiple testing adjusted $P$ values (with 95\% confidence interval to characterize estimation quality) were obtained by permutation test (described in

the supplementary Methods section).

${ }^{\mathrm{f}}$ Multivariate model.

gUnivariate phenotypes.

\section{STATISTICAL ANALYSES}

Statistical analyses were performed with commercially available software (GraphPad Prism, version 5.01; GraphPad Software Inc, La Jolla, California) for experimental data (animal study and expression experiments). For transfection studies and for PBMC analysis, 2-tailed pairwise Mann-Whitney test was applied; for mouse behavior studies, 2-way analysis of variance for repeated measures was used. For human data, haplotype association analyses of binary categorical variables was performed with UNPHASED (version 3.0.13; Frank Dudbridge, http://www.mrc-bsu.cam.ac.uk /personal/frank/software/unphased/), performing likelihood ratio tests in a log-linear model through unconditional logistic regression adjusting for age and sex. ${ }^{48}$ Correlations of the 3 quantitative modifier variables (executive functioning, reasoning, and verbal learning/memory) and the quantitative constitutive variable (premorbid intelligence) were assessed using Pearson productmoment correlation, ${ }^{49}$ determining internal consistency of the 3 target variables by the Cronbach $\alpha$ coefficient ${ }^{50}$ All metric phenotypic variables were standardized to be normally distributed with zero mean and variance 1 (by Blom Transformation ${ }^{51}$ [described in the supplementary Methods section]). Subsequently, linear models with covariate adjustment were used for singlemarker association analyses (R, version 2.8.1; http://cran.r-project .org) and for haplotype association analyses of the standardized quantitative traits (SAS, version 9.1 ${ }^{52}$ ), with PLINK (version 1.06$)^{53}$ for estimation and tabulation of all possible individuals' haplotype phases and their posterior probabilities (given the genotype data). Multivariate analysis modeled a target phenotype vector, accounting for individual correlation between vector entries. For single-marker association analyses, a permutation test was used to estimate multiple testing adjusted $P$ values to obtain by chance no less than the observed amount of significances for a given set of tests (Table). All other $P$ values are nominal, with $P$ less than .05 indicating significance. Details for quantitative trait analyses and for the permutation test are given in the supplementary Methods section.

\section{DATABASES}

Information on CPLX2 sequences was obtained from the National Center for Biotechnology Information (http://www.ncbi.nlm.nih .gov/) and the University of California-Santa Cruz (http://genome .ucsc.edu/) (GeneID, 10814). Accession numbers for the 2 CPLX2 transcripts are NM_006650.3 (T1) and NM_001008220.1 (T2).

\section{RESULTS}

\section{CPLX2 GENOTYPING AND ASSOCIATION STUDIES}

\section{Case-Control Study}

We analyzed first the genetic variability of the CPLX2 gene $^{54}$ in 1071 schizophrenic patients of the GRAS sample vs 1079 healthy control subjects with comparable ethnicity. The CPLX2 coding region (exons 4-6), adjacent introns, and 
A

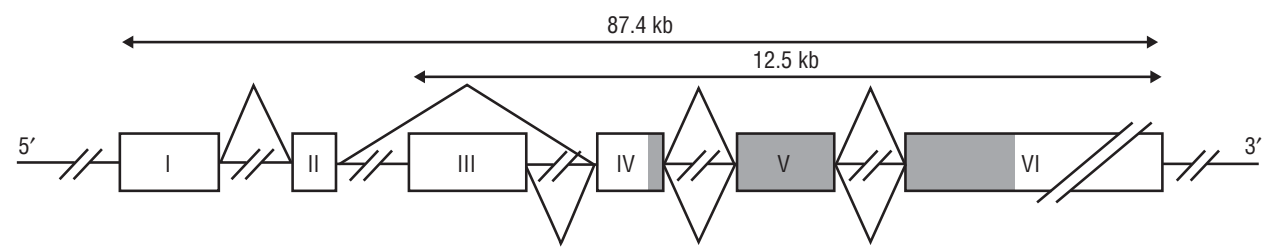

$B$
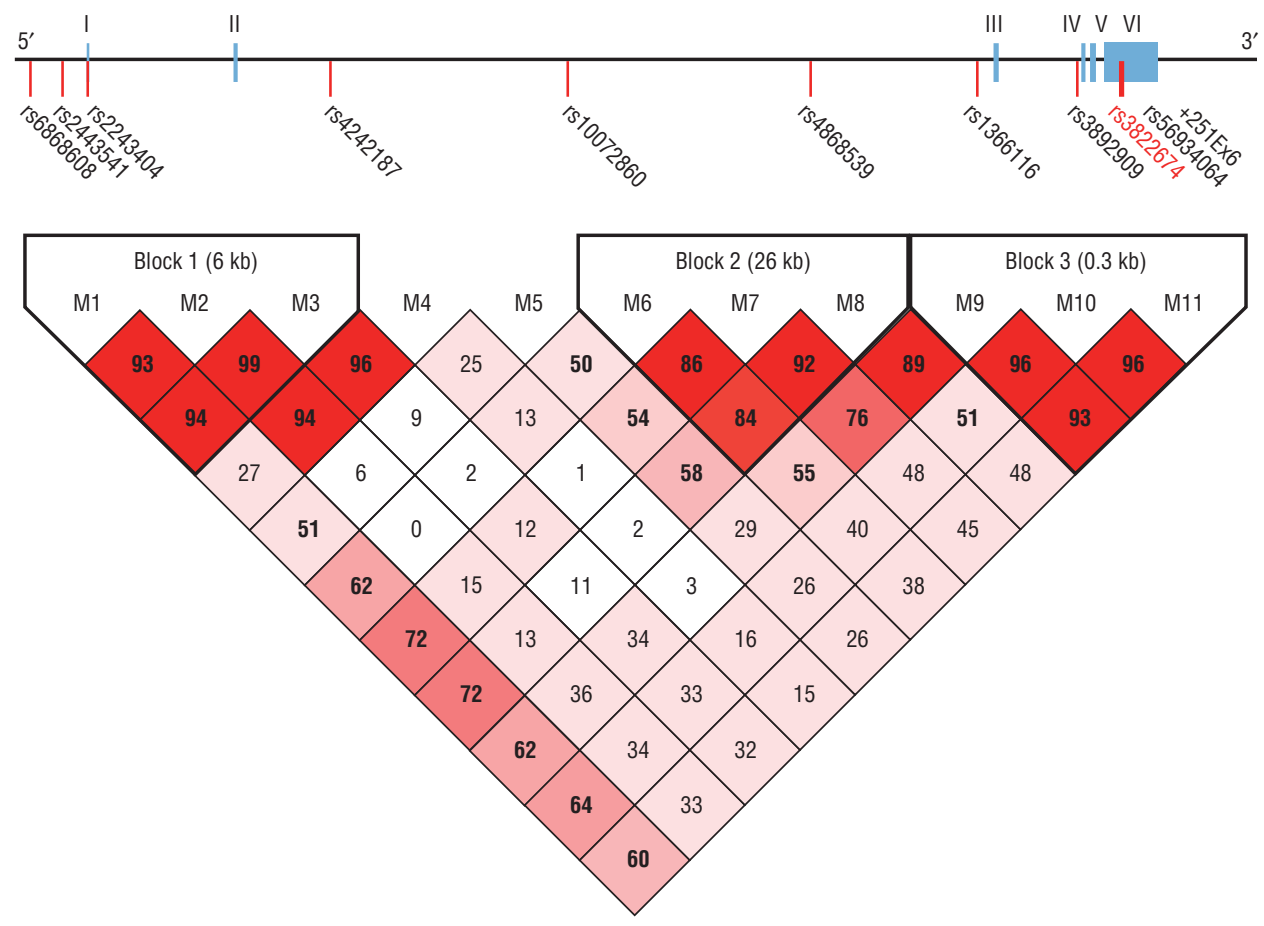

Figure 1. Genomic organization of the CPLX2 gene. A, The CPLX2 gene is located at $5 q 35.2$ and spans 87.4 kilobases (kb). The 6 exons (boxes) encode 2 different splicing variants (indicated by triangles) leading to 1 protein (coding region is shaded). Introns are shown as interrupted solid lines; size of transcripts is indicated. B, Overview of the analyzed markers (Ms) in the CPLX2 gene. The exons (blue boxes) are numbered and the single-nucleotide polymorphisms (SNPs) (red lines) are named (described in eTable 2). The SNP affecting the microRNA-498 binding site is labeled in red. Linkage disequilibrium (LD) analysis was performed in Haploview ${ }^{55}\left(n=2027\right.$ cases and control subjects). The numbers in the squares are the measure of LD, $D^{\prime}$. The darker the red is, the higher the linkage; all $\mathrm{D}$ values of 50 or higher are in boldface type. The gene shows 3 main blocks of high LD.

part of the 3'UTR were sequenced, revealing the presence of 4 SNPs in this region. The putative promoters, upstream of or within exons 1 and 3, and adjacent introns were analyzed by direct genotyping of 7 selected SNPs covering this region (Figure 1 and eTable 2).

On sequencing of the coding region of CPLX2, no informative mutations were found in schizophrenic or healthy control subjects (eTable 3). A simple case-control association study, based on end point diagnosis and single markers, did not yield significant differences between cases and controls regarding genotypic or allelic frequencies (eTable 4). Three main haploblocks, closely resembling those described for this region by the HapMap Project (http://www .hapmap.org), were also identified in our population (Figure 1). Here, a haplotypic combination covering these 3 haploblocks and consisting of SNPs rs2443541/rs3892909 $1+251 \mathrm{Ex} 6$ appeared to be increased in cases $(14.6 \%)$ vs controls $(12.0 \%)$ (odds ratio [OR], 1.31; 95\% confidence interval $\left.[\mathrm{CI}], 0.98-1.76 ; \chi_{1}^{2}=3.92 ; P=.048\right)$. This result, however, is of borderline significance and will need further confirmation. In contrast, a low-frequency haplotype
(2.3\% in cases vs $0.9 \%$ in controls) within haploblock 2 , including SNPs rs 1366116 and rs3892909, showed higher association with schizophrenia (OR, 2.47; 95\% CI, 1.41$\left.4.34 ; \chi_{1}^{2}=11.15 ; P<.001\right)$.

\section{Phenotype-Based Genetic Association Study}

In a first analysis of associations between genetic signatures and specific biological readouts within the GRAS group of schizophrenic patients, we focused on cognitive performance. Regarding the cognitive tests used, we followed our hypothesis of CPLX2 influencing current higher brain functions but not premorbid intelligence. This hypothesis was mainly derived from our Cplx2null mutant mouse study, exploring the effect of a second hit to the brain. Other neuropsychological tests performed in the frame of the GRAS project (which we looked at later for exploratory reasons) turned out to be less or not affected by CPLX2 gene variants (data not shown).

To examine the role of CPLX2 in cognition, we constructed a phenotypic intercorrelation network consist-

$$
\begin{gathered}
\hline \text { ARCH GEN PSYCHIATRY/VOL 67 (NO. 9), SEP } 2010 \quad \text { WWW.ARCHGENPSYCHIATRY.COM } \\
\mathbf{8 8 3} \\
\text { Downloaded from www.archgenpsychiatry.com on September 6, } 2010 \\
\text { O2010 American Medical Association. All rights reserved. } \\
\text { (REPRINTED WITH CORRECTIONS) }
\end{gathered}
$$




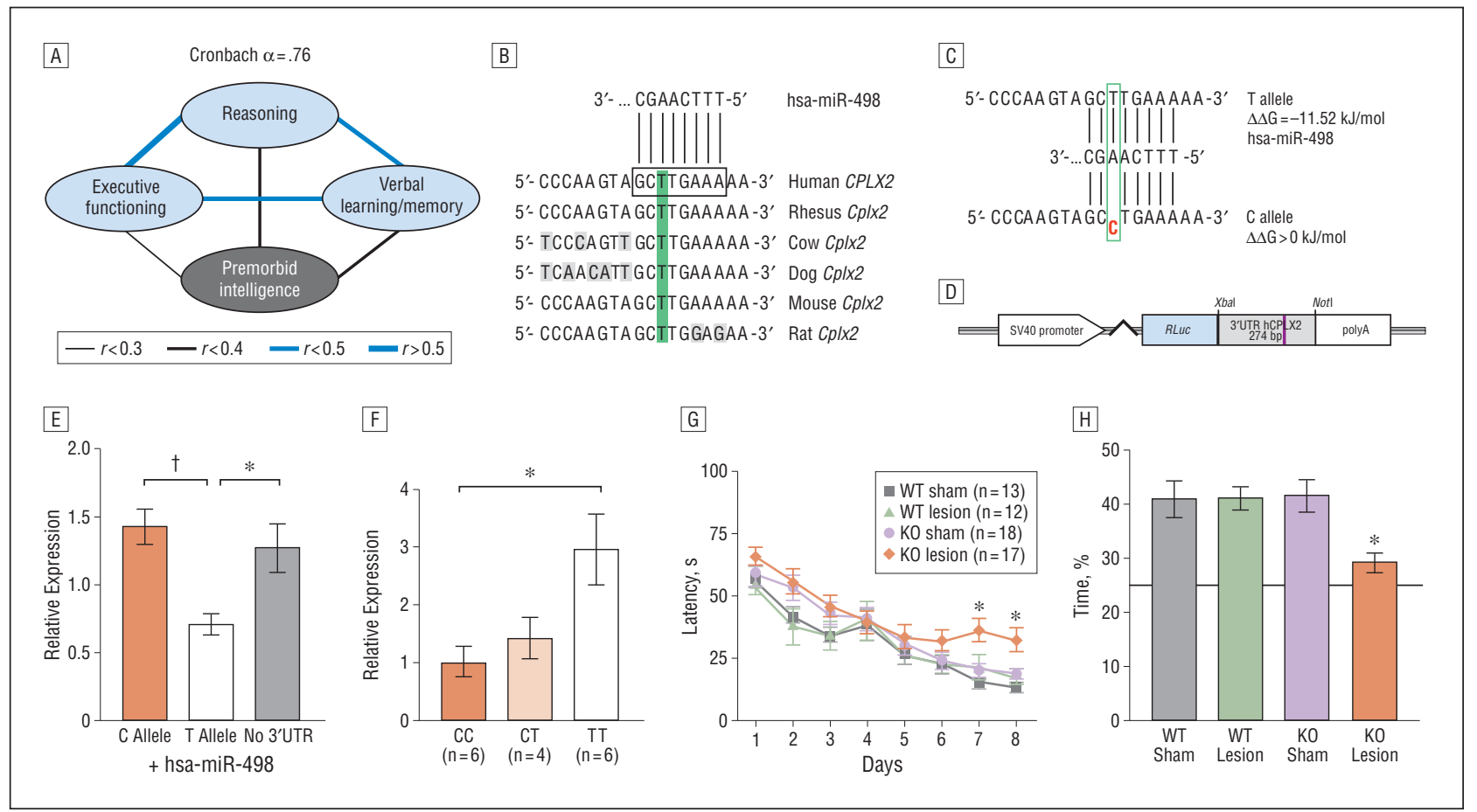

Figure 2. CPLX2 in schizophrenia: genotype-phenotype interactions. A, Intercorrelation network of target (light blue) and constitutive (gray) variables to demonstrate phenotype coherence regarding higher cognitive functions. The Cronbach $\alpha$ of .76 shows a high internal consistency of the 3 target variables. Lines of different thickness and color indicate strengths of correlation between different tests. B, The single-nucleotide polymorphism (SNP) rs3822674 (green shading) lies in the seed region (black box) of hsa-microRNA [miR]-498. Mismatches are indicated with gray shading. C, $\Delta \Delta \mathrm{G}$ values for the different alleles (green box) indicate the binding probabilities for hsa-miR-498 to the CPLX2 3' untranslated region (3'UTR). D, phRL-CPLX2-3'UTR contains an SV40 promoter, the Renilla luciferase $(R L u c)$ gene, the first 274 base pairs (bp) of the $3^{\prime} U T R$, and a polyA signal. The restriction sites are indicated and the pink line shows the predicted binding site for hsa-miR-498. E, phRL-CPLX2-3'UTR (containing C or T allele) or phRL-SV40 (without insert), internal control pCMV-FFLuc, and hsa-miR-498 were cotransfected into Neuro-2a (N2a) cells. Relative luciferase expression (normalized to the data without miR) is displayed for the different conditions. For 6 independent experiments, means were obtained of 6 replicates each. With pairwise Mann-Whitney 2 -tailed test, $* P=.015\left(U_{\mathrm{n} 1=\mathrm{n} 2=6}=3\right) ;+P=.002\left(U_{\mathrm{n} 1=\mathrm{n} 2=6}=0\right)$. $\mathrm{F}$, Spontaneous CPLX2 messenger RNA expression in peripheral blood mononuclear cells is modulated by the genotype. With pairwise Mann-Whitney 2 -tailed test, $* P=.026\left(U_{n 1=n 2=6}=4\right)$. G and $\mathrm{H}, \mathrm{A}$ "second hit" (cryolesion) is required to provoke a cognitive phenotype in Cp/x2-null mutant mice in the Morris water maze test. G, Only Cplx2-null mutant mice with lesions show elevated escape latency in the hidden platform task (2-way analysis of variance [ANOVA] for repeated measures applied for the last 2 test days; significant effect of factor lesion in $C p / x 2-$ null mutant mice: $\left.* F_{1,33}=10.83 ; P=.002\right)$. KO indicates knockout; WT, wild-type. $\mathrm{H}$, Again, only Cplx2-null mutant mice with lesions show absence of preference for the target quadrant in the probe trial (2-way ANOVA; significant interaction effect between factors genotype and lesion: $\left.* F_{1,56}=4.82 ; P=.032\right)$. In parts $\mathrm{E}$ through $\mathrm{H}$, limit lines represent SEM.

ing of test results for executive functioning, reasoning, and verbal learning/memory as target (modifier) variables that are subject to potential influence of disease-modifying factors. Premorbid intelligence, representing the development dependent intellectual state at disease onset, was selected as the constitutive variable, expected to influence the cognitive phenotype and to correlate with the target variables but to be essentially independently regulated (Figure 2A). Determination of premorbid intelligence assists in estimation of the cognitive decline in brain diseases such as schizophrenia. The most frequently used tests (including the one applied herein, the MehrfachwahlWortschatz Test $\mathrm{B}^{56}$ ) measure vocabulary skills, which essentially depend on the level of education reached at the time point of disease onset. ${ }^{57}$ This vocabulary knowledge, once acquired, tends to stay on (frozen) even if other cognitive skills decline owing to the disease process. As expected, there is a highly significant correlation between the number of years in school according to the final degree as a measure of level of education and the test results of the Mehrfachwahl-Wortschatz Test B (in the GRAS sample at present, $r=0.45 ; P<.001)$.

The cognitive target variables together yielded a phenotypic intercorrelation network of high quality (Cron- bach $\alpha=.76$ ), providing a solid basis for multivariate analyses. After correction for age and sex, 4 SNPs in the CPLX2 gene (among them 2 markers belonging to the haplotypes of risk described in the case-control approach) correlated with overall neurocognitive function as evaluated by our multivariate model. Tests of univariate phenotypes-executive functioning, reasoning, and verbal learning/memory-showed this association even for 6 SNPs (Table). As expected, the constitutive variable premorbid intelligence was not significantly associated with single SNPs. The Table provides a synopsis of all tested SNPs of CPLX2 for the multivariate model and the univariate phenotypes, as well as for the constitutive variable. Global functioning is presented as a diseaserelevant control variable. Raw data and the influence of specific genotypes on cognition are shown in eFigure 1 and eTable 5.

Multilocus analysis, including all SNPs with any effects on cognition in individual neuropsychological tests (Table), showed that the combination of markers has a statistically significant effect on cognitive performance in the multivariate model and the univariate phenotypes (eTable 6). The haplotypic combination CTC at markers rs1366116/rs3892909/rs3822674 predicts the

\footnotetext{
ARCH GEN PSYCHIATRY/VOL 67 (NO. 9), SEP 2010 WWW.ARCHGENPSYCHIATRY.COM 
worst cognitive outcome for all target phenotypes and even for the constitutive variable. In fact, the CTC haplotype shows significantly decreased cognitive performance compared with all other haplotypic combinations (eTable 7).

\section{SEARCH FOR MECHANISMS OF SNP FUNCTION}

\section{Allele-Dependent Reporter Gene Expression}

To gain a first insight into the mechanisms by which the identified SNPs might modulate certain domains of cognition, we performed an extensive analysis of the relevant genomic sequences (see the supplementary Methods section). One of the cognition-relevant SNPs that we identified (rs3822674) is located within a predicted binding site of the hsa-miR-498 in the 3'UTR of the CPLX2 mRNA. This binding site is highly conserved among species (Figure 2B and $\mathrm{C}$ as well as eFigure 2 and eTable 7 ). Allele-dependent structural predictions and $\Delta \Delta G$ values indicated that the $\mathrm{C}$-to- $\mathrm{T}$ exchange affects miR binding and might therefore modulate CPLX2 expression. To test this, we cloned the first $274 \mathrm{bp}$ of the 3'UTR of CPLX2 downstream of a luciferase reporter gene (phRL-SV40; Figure 2D) and transfected this construct into N2a cells. On addition of hsa-miR-498, luciferase expression was significantly ( $>50 \%$ ) reduced in the presence of the T allele $(U=0.00 ; P=.002)$, whereas the presence of the $C$ allele yielded an expression comparable to that of the control (vector lacking 3'UTR insert) (Figure 2E). These results suggest a role of SNP rs3822674 in posttranscriptional regulation of CPLX2 expression that may become relevant on miR profile changes in specific neuronal subsets in response to, for example, brain injury. ${ }^{38}$

\section{Spontaneous Genotype-Dependent CPLX2 mRNA Expression in PBMCs}

To test the influence of genotype on baseline CPLX2 mRNA levels, PBMCs from patients with the CC, CT, or TT genotype at SNP rs3822674 were analyzed. There was a significant genotype effect, with the TT genotype having the highest levels of the transcript (Figure 2F).

\section{MODELING COGNITIVE EFFECTS OF ALTERED Cplx2 EXPRESSION IN MICE}

Based on the hypothesis that altered CPLX2 expression influences cognitive performance, we investigated mice with a $C p l \times 2$-null mutation. ${ }^{58}$ These mice develop essentially normally and lack major behavioral abnormalities. To test for a potential influence of a second hit to their brains, a right parietal cortical cryolesion was applied stereotactically at a vulnerable time (ie, puberty [day 28 of life]). This lesion paradigm had originally been developed to model the neurodegenerative processes of schizophrenia ${ }^{47}$ spreading from the initiation site, the parietal lobe, to other cortical areas. ${ }^{59}$ Specific deficits in spatial memory (Morris water maze, escape latency) became evident only in Cplx2-null mutant mice that had received a peripubertal lesion $\left(F_{1,33}=10.83 ; P=.002\right)$, but not in identically treated wild-type littermates $\left(F_{1,23}=2.27\right.$; $P=.146$ ) (Figure 2G). Also, only lesioned Cplx2-null mutant mice showed absence of any preference for the target quadrant (Morris water maze, probe trial, $F_{1,56}=4.82$; $P=.032$ ) (Figure $2 \mathrm{H}$ ). Hence, a cognition modifier role of $C p l \times 2$ was revealed only upon combination of a Cplx2null mutation with an environmental cofactor (ie, parietal cryolesion as a second hit), leading to a remarkable deterioration of cognitive performance.

\section{COMMENT}

Collectively, these data support a modifier role of CPLX2 variants on cognitive performance in schizophrenia. Whereas our conventional case-control study revealed schizophrenia-at-risk haplotypes of the CPLX2 gene, constituting several of the investigated markers, only the PGAS allowed the specific identification of 6 cognitionrelated SNPs. These SNPs in turn may become relevant mainly on additional (environmental) cofactors, that is, second hits. The effects of CPLX2 genotypes on current cognition of schizophrenic patients observed herein do not exclude a comparable role in other neuropsychiatric disorders or even in healthy individuals in which, for instance, aging would be an inevitable second hit. In this regard, further extensive studies on different populations are required. Among the mechanisms mediating genotype-dependent CPLX2 expression upon second hit may be the binding of hsa-miR-498 to SNP rs3822674 in the 3'UTR of the CPLX2 mRNA that leads to its subsequent downregulation.

Measuring spontaneous CPLX2 mRNA expression in PBMCs of schizophrenic patients reveals different levels dependent on the genotype, with the best cognitive performers (TT at SNP rs3822674) showing the highest expression. In contrast, the CC carriers have lower mRNA expression and reduced cognitive capabilities. Most important, however, CC and TT carriers are not different when compared with respect to their premorbid intelligence, reflecting cognitive abilities before they were "hit by the disease." Correspondingly, Cplx2-null mutant mice are severely cognitively impaired only after a second hit, delivered in the present study by mild neurotrauma (juvenile parietal cortical cryolesion) and in a prior report by maternal deprivation stress. ${ }^{34}$ Intriguingly, only the $\mathrm{T}$ allele at SNP rs3822674 (resulting in higher baseline levels of CPLX2 mRNA and better cognition) allows binding of hsa-miR-498 and thus regulability, that is, subsequent downregulation of CPLX2 mRNA.

Current models of a dichotomous Cplx function assume that Cplxs have a facilitatory role in transmitter release, by stabilizing SNARE complexes in a highly fusogenic state, as well as an inhibitory role, by clamping SNARE complexes and thus preventing them from executing synaptic vesicle fusion until triggered by an action potential and the concomitant increase in the intrasynaptic calcium ion concentration. Indeed, calcium ion-regulated exocytosis in many different preparations is inhibited to similar degrees by increased or decreased Cplx activity. ${ }^{21,27}$

\footnotetext{
$\overline{\text { ARCH GEN PSYCHIATRY/VOL } 67 \text { (NO. 9), SEP } 2010 \text { WWW.ARCHGENPSYCHIATRY.COM }}$ 
Based on these facts and the cognitive data from our patients, we conclude that the regulability necessary for maintaining or adjusting the homeostasis of CPLX2 expression may constitute a key factor in the fine tuning of synaptic function. This concept has similarly been suggested earlier on the basis of in vitro studies ${ }^{60}$ and is in agreement with the notions that (1) loss as well as overexpression of CPLXs/Cplxs can perturb presynaptic secretory function, and that (2) CPLXs combine facilitatory and inhibitory functions with respect to synaptic secretion. This functional combination has been delineated in a recent study also demonstrating that in different CPLXs the balance between facilitatory and inhibitory activities may be different. ${ }^{61}$

Considering the large number of human postmortem studies describing abnormalities in the absolute amount or the ratios of the different CPLX proteins and CPLX mRNAs, ${ }^{13-18,31}$ these dichotomous CPLX/ Cplx functions and their tight regulation under physiological conditions may be of major relevance for pathological cognition seen in several neuropsychiatric diseases. The mechanistic basis for the observed alterations in tissue concentration, however, is presently far from clear.

Analyses of mouse models failed to provide evidence of simple compensatory changes in Cplx expression in the corresponding Cplx1-, Cplx2-, Cplx3-, or Cplx4single-null mutant mice. ${ }^{58,62,63}$ Expression levels of all other presynaptic proteins tested so far were also found to be unaltered in Cplxl-null mutant brains ( $\alpha$-SNAP, Munc13-1, Munc13-2, Munc13-3, Munc18-1, Nethylmaleimide-sensitive factor, SNAP-25, synapsin I/Ila, synapsin IIb, synaptobrevin 2 , synaptophysin, synaptotagmin 1 , syntaxin 1 , and vesicular $\gamma$-aminobutyric acid transporter), in Cplx2-null mutant brains ( $\alpha$-SNAP, Munc13-1, Munc13-2, Munc13-3, Munc18-1, SNAP25 , synapsin I/IIa, synapsin IIb, synaptobrevin 2 , synaptophysin, synaptotagmin 1, and syntaxin 1), and in Cplx1/ 2-double-null mutant brains (SNAP-25, synaptobrevin 2 , synaptotagmin 1 , syntaxin 1 , and vesicular $\gamma$ aminobutyric acid transporter).$^{58}$ In Cplx3/4-doublenull mutant retina, only Ribeye expression was reduced among 17 presynaptic proteins tested. ${ }^{62}$ Based on these findings, we would argue that even loss of CPLX2/ Cplx2 is unlikely to be compensated for by robust changes in the expression levels of other related presynaptic proteins. Functionally relevant alterations, however, may be subtle and escape detection by the available methods. Also, given that pathological states were not explored and that not all of the many dozens of proteins were tested that might act in a compensatory manner on loss of Cplx2, the possibility remains that such compensatory changes occur. However, it is almost impossible to investigate this systematically with the currently available technology and tools/reagents.

In addition to SNP rs3822674, 5 other SNPs were also associated with cognitive performance of schizophrenic patients in the present study. The mechanisms underlying the impact of these other intronic genetic variants on cognition are still unclear, but several possibilities may be considered. (1) Because of the linkage disequilibrium between these genetic markers, the influence of the 3'UTR regulatory mechanism mediated by SNP rs3822674 may be detected by several other markers along the gene. (2) Effects of the intronic variants, largely independent of the rs3822674 3'UTR mechanism, cannot be excluded at this point, for example, on transcription factor binding sites or other regulatory elements in the gene affecting expression level or splicing of transcripts.

To summarize, we propose that neurons in brain regions that control cognitive abilities requiredepending on the situation - an exact control of CPLX2 expression. Subtle disturbances in the optimal amount of CPLX2/Cplx2 levels would therefore influence cognitive functions. Our data indicate (1) that this control is, at least in part, mediated by the binding of hsa-miR498 to the 3'UTR of the CPLX2 mRNA, and (2) that a polymorphism in the binding region of hsa-miR-498 (SNP rs3822674) influences CPLX2 gene expression and thus modulates cognitive performance. Together with other genetic and environmental mechanisms that affect cognition, this genetic variant may be an important codeterminant of cognitive outcome in schizophrenia.

Submitted for Publication: October 12, 2009; final revision received January 19, 2010; accepted February 24, 2010.

Author Affiliations: Division of Clinical Neuroscience (Drs Begemann, Krampe, Radyushkin, Ghorbani, Gutwinski, Hilmes, Leppert, Stawicki, Stödtke, Szuszies, and Ehrenreich; Mss Grube, Ribbe, Friedrichs, Hannke, Sperling, Ronnenberg, and Sowislo; and Messrs El-Kordi and Gerchen) and Departments of Neurogenetics (Drs Papiol, Eckstein, and Nave) and Molecular Neurobiology (Drs Reim and Brose, Mr Benseler, and Mss Schwerdtfeger and Thanhäuser), Max Planck Institute of Experimental Medicine; Departments of Genetic Epidemiology (Drs Malzahn and Bickeböller), Transfusion Medicine (Dr Riggert), and Psychiatry and Psychotherapy (Dr Falkai), Georg-August-University, Göttingen, Germany.

Correspondence: Hannelore Ehrenreich, MD, DVM, Division of Clinical Neuroscience, Max Planck Institute of Experimental Medicine, Hermann-Rein Str 3, 37075 Göttingen, Germany (ehrenreich@em.mpg.de), or Nils Brose, $\mathrm{PhD}$, Department of Molecular Neurobiology, Max Planck Institute of Experimental Medicine, Hermann-Rein Str 3, 37075 Göttingen, Germany (brose@em.mpg.de).

Author Contributions: Dr Begemann and Ms Grube contributed equally to this work.

Financial Disclosure: None reported.

Funding/Support: This study was supported by the Max Planck Society, by the DFG-Research Center for Molecular Physiology of the Brain, and by several private donations. All support was devoted to the GRAS project under the leadership of Dr Ehrenreich.

Online-Only Material: The supplementary Appendix, Methods section, eTables, and eFigures are available at http://www.archgenpsychiatry.com.

Additional Contributions: We thank all the patients for their participation in this study and all the collaborating centers for their support.

\footnotetext{
$\overline{\text { ARCH GEN PSYCHIATRY/VOL } 67 \text { (NO. 9), SEP } 2010 \text { WWW.ARCHGENPSYCHIATRY.COM }}$ 


\section{REFERENCES}

1. Andreasen NC. Symptoms, signs, and diagnosis of schizophrenia. Lancet. 1995; 346(8973):477-481.

2. Burmeister M, McInnis MG, Zöllner S. Psychiatric genetics: progress amid controversy. Nat Rev Genet. 2008;9(7):527-540.

3. Purcell SM, Wray NR, Stone JL, Visscher PM, O'Donovan MC, Sullivan PF, Sklar $\mathrm{P}$; International Schizophrenia Consortium. Common polygenic variation contributes to risk of schizophrenia and bipolar disorder. Nature. 2009;460(7256): 748-752.

4. Shi J, Levinson DF, Duan J, Sanders AR, Zheng Y, Pe'er I, Dudbridge F, Holmans PA, Whittemore AS, Mowry BJ, Olincy A, Amin F, Cloninger CR, Silverman JM, Buccola NG, Byerley WF, Black DW, Crowe RR, Oksenberg JR, Mirel DB, Kendler KS, Freedman R, Gejman PV. Common variants on chromosome 6p22.1 are associated with schizophrenia. Nature. 2009;460(7256):753-757.

5. Stefansson H, Ophoff RA, Steinberg S, Andreassen OA, Cichon S, Rujescu D, Werge T, Pietiläinen OP, Mors O, Mortensen PB, Sigurdsson E, Gustafsson 0, Nyegaard M, Tuulio-Henriksson A, Ingason A, Hansen T, Suvisaari J, Lonnqvist J, Paunio T, Børglum AD, Hartmann A, Fink-Jensen A, Nordentoft M, Hougaard D, Norgaard-Pedersen B, Böttcher Y, Olesen J, Breuer R, Möller HJ, Giegling I, Rasmussen HB, Timm S, Mattheisen M, Bitter I, Réthelyi JM, Magnusdottir BB, Sigmundsson T, Olason P, Masson G, Gulcher JR, Haraldsson M, Fossdal R, Thorgeirsson TE, Thorsteinsdottir U, Ruggeri M, Tosato S, Franke B, Strengman E, Kiemeney LA, Melle I, Djurovic S, Abramova L, Kaleda V, Sanjuan J, de Frutos R, Bramon E, Vassos E, Fraser G, Ettinger U, Picchioni M, Walker N, Toulopoulou T, Need AC, Ge D, Yoon JL, Shianna KV, Freimer NB, Cantor RM, Murray R, Kong A, Golimbet V, Carracedo A, Arango C, Costas J, Jönsson EG, Terenius L, Agartz I, Petursson H, Nöthen MM, Rietschel M, Matthews PM, Muglia P, Peltonen L, St Clair D, Goldstein DB, Stefansson K, Collier DA; Genetic Risk and Outcome in Psychosis (GROUP). Common variants conferring risk of schizophrenia. Nature. 2009;460 (7256):744-747.

6. Reichenberg A, Harvey PD. Neuropsychological impairments in schizophrenia: Integration of performance-based and brain imaging findings [published correction appears in Psychol Bull. 2008;134(3):382]. Psychol Bull. 2007;133 (5):833-858.

7. Mirnics K, Middleton FA, Lewis DA, Levitt P. Analysis of complex brain disorders with gene expression microarrays: schizophrenia as a disease of the synapse. Trends Neurosci. 2001;24(8):479-486.

8. Harrison PJ, Weinberger DR. Schizophrenia genes, gene expression, and neuropathology: on the matter of their convergence [published corrections appear in Mol Psychiatry. 2005;10(4):420, and Mol Psychiatry. 2005;10(8):804]. Mol Psychiatry. 2005;10(1):40-68.

9. Owen MJ, O'Donovan MC, Harrison PJ. Schizophrenia: a genetic disorder of the synapse? BMJ. 2005;330(7484):158-159.

10. Karlsgodt KH, Sun D, Jimenez AM, Lutkenhoff ES, Willhite R, van Erp TG, Cannon TD. Developmental disruptions in neural connectivity in the pathophysiology of schizophrenia. Dev Psychopathol. 2008;20(4):1297-1327.

11. Stephan KE, Friston KJ, Frith CD. Dysconnection in schizophrenia: from abnormal synaptic plasticity to failures of self-monitoring. Schizophr Bull. 2009; 35(3):509-527.

12. McMahon HT, Missler M, Li C, Südhof TC. Complexins: cytosolic proteins that regulate SNAP receptor function. Cell. 1995;83(1):111-119.

13. Harrison PJ, Eastwood SL. Preferential involvement of excitatory neurons in medial temporal lobe in schizophrenia. Lancet. 1998;352(9141):1669-1673.

14. Eastwood SL, Harrison PJ. Hippocampal synaptic pathology in schizophrenia, bipolar disorder and major depression: a study of complexin mRNAs. Mol Psychiatry. 2000;5(4):425-432.

15. Eastwood SL, Cotter D, Harrison PJ. Cerebellar synaptic protein expression in schizophrenia. Neuroscience. 2001;105(1):219-229.

16. Sawada K, Young CE, Barr AM, Longworth K, Takahashi S, Arango V, Mann JJ, Dwork AJ, Falkai P, Phillips AG, Honer WG. Altered immunoreactivity of complexin protein in prefrontal cortex in severe mental illness. Mol Psychiatry. 2002; 7(5):484-492.

17. Knable MB, Barci BM, Webster MJ, Meador-Woodruff J, Torrey EF; Stanley Neuropathology Consortium. Molecular abnormalities of the hippocampus in severe psychiatric illness: postmortem findings from the Stanley Neuropathology Consortium. Mol Psychiatry. 2004;9(6):609-620.

18. Eastwood SL, Harrison PJ. Decreased expression of vesicular glutamate transporter 1 and complexin II mRNAs in schizophrenia: further evidence for a synaptic pathology affecting glutamate neurons. Schizophr Res. 2005;73(2-3): 159-172.

19. Reim K, Wegmeyer H, Brandstätter JH, Xue M, Rosenmund C, Dresbach T, Hofmann K, Brose N. Structurally and functionally unique complexins at retinal ribbon synapses. J Cell Biol. 2005;169(4):669-680.
20. Brose N. For better or for worse: complexins regulate SNARE function and vesicle fusion. Traffic. 2008;9(9):1403-1413.

21. Südhof TC, Rothman JE. Membrane fusion: grappling with SNARE and SM proteins. Science. 2009;323(5913):474-477.

22. Takahashi S, Ujihara H, Huang GZ, Yagyu KI, Sanbo M, Kaba H, Yagi T. Reduced hippocampal LTP in mice lacking a presynaptic protein: complexin II. Eur J Neurosci. 1999;11(7):2359-2366.

23. Gibson HE, Reim K, Brose N, Morton AJ, Jones S. A similar impairment in CA3 mossy fibre LTP in the R6/2 mouse model of Huntington's disease and in the complexin II knockout mouse. Eur J Neurosci. 2005;22(7):1701-1712.

24. Strenzke N, Chanda S, Kopp-Scheinpflug C, Khimich D, Reim K, Bulankina AV, Neef A, Wolf F, Brose N, Xu-Friedman MA, Moser T. Complexin-l is required for high-fidelity transmission at the endbulb of held auditory synapse. J Neurosci. 2009;29(25):7991-8004.

25. Lee HJ, Song JY, Kim JW, Jin SY, Hong MS, Park JK, Chung JH, Shibata H, Fukumaki Y. Association study of polymorphisms in synaptic vesicle-associated genes, SYN2 and CPLX2, with schizophrenia. Behav Brain Funct. 2005;1:15.

26. Kishi T, Ikeda M, Suzuki T, Kitajima T, Yamanouchi Y, Kinoshita Y, Ozaki N, Iwata N. No association of complexin1 and complexin2 genes with schizophrenia in a Japanese population. Schizophr Res. 2006;82(2-3):185-189.

27. Brose N. Altered complexin expression in psychiatric and neurological disorders: cause or consequence? Mol Cells. 2008;25(1):7-19.

28. Morton AJ, Faull RL, Edwardson JM. Abnormalities in the synaptic vesicle fusion machinery in Huntington's disease. Brain Res Bull. 2001;56(2):111-117.

29. DiProspero NA, Chen EY, Charles V, Plomann M, Kordower JH, Tagle DA. Early changes in Huntington's disease patient brains involve alterations in cytoskeletal and synaptic elements. J Neurocytol. 2004;33(5):517-533.

30. Tannenberg RK, Scott HL, Tannenberg AE, Dodd PR. Selective loss of synaptic proteins in Alzheimer's disease: evidence for an increased severity with APOE ع4. Neurochem Int. 2006;49(7):631-639.

31. Sawada K, Barr AM, Nakamura M, Arima K, Young CE, Dwork AJ, Falkai P, Phillips AG, Honer WG. Hippocampal complexin proteins and cognitive dysfunction in schizophrenia. Arch Gen Psychiatry. 2005;62(3):263-272.

32. Drew CJ, Kyd RJ, Morton AJ. Complexin 1 knockout mice exhibit marked deficits in social behaviours but appear to be cognitively normal. Hum Mol Genet. 2007;16(19):2288-2305.

33. Glynn D, Bortnick RA, Morton AJ. Complexin II is essential for normal neurological function in mice. Hum Mol Genet. 2003;12(19):2431-2448.

34. Yamauchi Y, Qin LH, Nishihara M, Sawada K, Kato K, Inoue S. Vulnerability of synaptic plasticity in the complexin II knockout mouse to maternal deprivation stress. Brain Res. 2005;1056(1):59-67.

35. Glynn D, Reim K, Brose N, Morton AJ. Depletion of Complexin II does not affect disease progression in a mouse model of Huntington's disease (HD); support for role for complexin II in behavioural pathology in a mouse model of HD. Brain Res Bull. 2007;72(2-3):108-120.

36. Oliver PL, Davies KE. Interaction between environmental and genetic factors modulates schizophrenic endophenotypes in the Snap-25 mouse mutant blind-drunk. Hum Mol Genet. 2009;18(23):4576-4589.

37. Bicker S, Schratt G. MicroRNAs: tiny regulators of synapse function in development and disease. J Cell Mol Med. 2008;12(5A):1466-1476.

38. Hébert SS, De Strooper B. Alterations of the microRNA network cause neurodegenerative disease. Trends Neurosci. 2009;32(4):199-206.

39. Ashcroft R. The Declaration of Helsinki. In: Emanuel E, Grady C, Crouch R, eds. The Oxford Textbook of Clinical Research Ethics. New York, NY: Oxford University Press; 2007:141-149.

40. American Psychiatric Association. Diagnostic and Statistical Manual of Mental Disorders. 4th ed. Washington, DC: American Psychiatric Association; 1994.

41. Wittchen H-U, Zaudig M, Fydrich T. SCID-I: Structured Clinical Interview for DSM-IV Disorders. Goettingen, Germany: Hogrefe; 1997.

42. McGlashan TH, Fenton WS. Classical subtypes for schizophrenia: literature review for DSM-IV. Schizophr Bull. 1991;17(4):609-632.

43. Jakobsson M, Scholz SW, Scheet P, Gibbs JR, VanLiere JM, Fung HC, Szpiech ZA, Degnan JH, Wang K, Guerreiro R, Bras JM, Schymick JC, Hernandez DG, Traynor BJ, Simon-Sanchez J, Matarin M, Britton A, van de Leemput J, Rafferty I, Bucan M, Cann HM, Hardy JA, Rosenberg NA, Singleton AB. Genotype, haplotype and copy-number variation in worldwide human populations. Nature. 2008; 451(7181):998-1003.

44. Li JZ, Absher DM, Tang H, Southwick AM, Casto AM, Ramachandran S, Cann HM, Barsh GS, Feldman M, Cavalli-Sforza LL, Myers RM. Worldwide human relationships inferred from genome-wide patterns of variation. Science. 2008;319(5866): 1100-1104.

45. Xing J, Watkins WS, Witherspoon DJ, Zhang Y, Guthery SL, Thara R, Mowry BJ, Bulayeva $\mathrm{K}$, Weiss RB, Jorde LB. Fine-scaled human genetic structure revealed by SNP microarrays. Genome Res. 2009;19(5):815-825. 
46. Steffens M, Lamina C, Illig T, Bettecken T, Vogler R, Entz P, Suk EK, Toliat MR, Klopp N, Caliebe A, König IR, Köhler K, Ludemann J, Diaz Lacava A, Fimmers R, Lichtner P, Ziegler A, Wolf A, Krawczak M, Nūrnberg P, Hampe J, Schreiber S, Meitinger T, Wichmann HE, Roeder K, Wienker TF, Baur MP. SNP-based analysis of genetic substructure in the German population. Hum Hered. 2006;62 (1):20-29.

47. Sirén AL, Radyushkin K, Boretius S, Kämmer D, Riechers CC, Natt O, Sargin D, Watanabe T, Sperling S, Michaelis T, Price J, Meyer B, Frahm J, Ehrenreich H. Global brain atrophy after unilateral parietal lesion and its prevention by erythropoietin. Brain. 2006;129(pt 2):480-489.

48. Dudbridge F. Likelihood-based association analysis for nuclear families and unrelated subjects with missing genotype data. Hum Hered. 2008;66(2): 87-98.

49. Armitage P, Berry G, Matthews JNS. Statistical Methods in Medical Research. 4th ed. Oxford, England: Blackwell; 2002.

50. Cronbach LJ. Coefficient alpha and the internal structure of tests. Psychometrika. 1951;16(3):297-334.

51. Blom G. Statistical Estimates and Transformed Beta Variables. New York, NY: John Wiley \& Sons Inc; 1958.

52. SAS Institute, Inc. SAS Software, Version 9.1 of the SAS System for Windows. Cary, NC: SAS Institute Inc; 2001.

53. Purcell S, Neale B, Todd-Brown K, Thomas L, Ferreira MA, Bender D, Maller J, Sklar P, de Bakker PI, Daly MJ, Sham PC. PLINK: a tool set for whole-genome association and population-based linkage analyses. Am J Hum Genet. 2007; 81(3):559-575.

54. Raevskaya NM, Dergunova LV, Vladychenskaya IP, Stavchansky VV, Oborina MV Poltaraus AB, Limborska SA. Structural organization of the human complexin 2 gene (CPLX2) and aspects of its functional activity. Gene. 2005;359:127137.
55. Barrett JC, Fry B, Maller J, Daly MJ. Haploview: analysis and visualization of LD and haplotype maps. Bioinformatics. 2005;21(2):263-265.

56. Lehrl S, Triebig G, Fischer B. Multiple choice vocabulary test MWT as a valid and short test to estimate premorbid intelligence. Acta Neurol Scand. 1995;91(5): 335-345.

57. Woodberry KA, Giuliano AJ, Seidman LJ. Premorbid IQ in schizophrenia: a metaanalytic review. Am J Psychiatry. 2008;165(5):579-587.

58. Reim K, Mansour M, Varoqueaux F, McMahon HT, Südhof TC, Brose N, Rosenmund C. Complexins regulate a late step in $\mathrm{Ca}^{2+}$-dependent neurotransmitter release. Cell. 2001;104(1):71-81.

59. Thompson PM, Vidal C, Giedd JN, Gochman P, Blumenthal J, Nicolson R, Toga AW, Rapoport JL. Mapping adolescent brain change reveals dynamic wave of accelerated gray matter loss in very early-onset schizophrenia. Proc Natl Acad Sci U S A. 2001;98(20):11650-11655.

60. Edwardson JM, Wang CT, Gong B, Wyttenbach A, Bai J, Jackson MB, Chapman ER, Morton AJ. Expression of mutant huntingtin blocks exocytosis in PC12 cells by depletion of complexin II. J Biol Chem. 2003;278(33):30849-30853.

61. Xue M, Lin YQ, Pan H, Reim K, Deng H, Bellen HJ, Rosenmund C. Tilting the balance between facilitatory and inhibitory functions of mammalian and Drosophila complexins orchestrates synaptic vesicle exocytosis. Neuron. 2009; 64(3):367-380.

62. Reim K, Regus-Leidig H, Ammermüller J, El-Kordi A, Radyushkin K, Ehrenreich $\mathrm{H}$, Brandstätter $\mathrm{JH}$, Brose N. Aberrant function and structure of retinal ribbon synapses in the absence of complexin 3 and complexin 4. J Cell Sci. 2009; 122(Pt 9):1352-1361.

63. Xue M, Stradomska A, Chen H, Brose N, Zhang W, Rosenmund C, Reim K. Complexins facilitate neurotransmitter release at excitatory and inhibitory synapses in mammalian central nervous system. Proc Natl Acad Sci U S A. 2008; 105(22):7875-7880. 


\section{Supplementary Online Content}

Begemann M, Grube S, Papiol S, Malzahn D, Krampe H, Ribbe K, Friedrichs H, Radyushkin KA, El-Kordi A, Benseler F, Hannke K, Sperling S, Schwerdtfeger D, Thanhäuser I, Gerchen MF, Ghorbani M, Gutwinski S, Hilmes C, Leppert R, Ronnenberg A, Sowislo J, Stawicki S, Stödtke M, Szuszies C, Reim K, Riggert J, Eckstein F, Falkai P, Bickeböller H, Nave K-A, Brose N, Ehrenreich $\mathrm{H}$. Modification of cognitive performance in schizophrenia by complexin 2 gene polymorphisms. Arch Gen Psychiatry. 2010;67(9):879-888.

eAppendix. Collaborating Centers

eMethods. Supplementary Methods Description

eTable 1. Allele-Specific Melting Temperatures for Simple Probe Assays

eTable 2. Characteristics of Analyzed SNPs

eTable 3. Point Mutations Detected in the Sequenced Region of CPLX2

eTable 4. Frequencies of Single SNP Analysis (Case-Control)

eTable 5. Pairwise Association Test on Cognitive Measures of Schizophrenic Patients

eTable 6. Multilocus Association Analysis for CPLX2

eTable 7. Association Analysis of CPLX2, Testing the Effect of One Regional Variant

(CTC Haplotype in CPLX2 Haploblocks 2 \& 3) Adjusted for All Other Associated CPLX2 Regions

eTable 8. Species Conservation

eFigure 1. Influence of CPLX2 Genotypes on Cognitive Measures of Schizophrenic Patients

eFigure 2. Conservation Status of the CPLX2 Gene Across 28 Species

eReferences. Bibliography for the Supplementary Methods Section

This supplementary material has been provided by the authors to give readers additional information about their work. 


\section{eAppendix. Collaborating Centers}

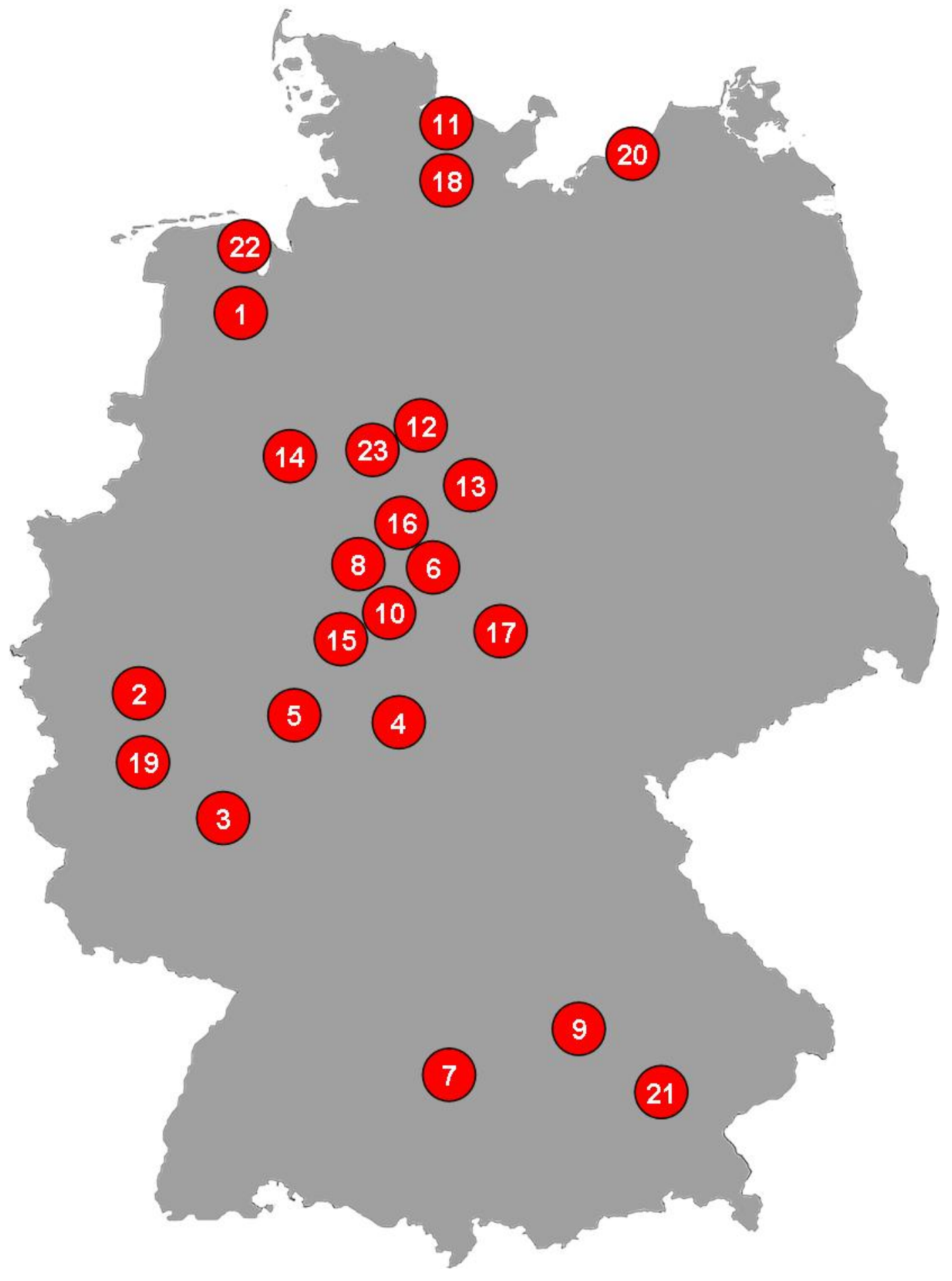




\begin{tabular}{|c|c|c|c|}
\hline No. & Center & Institution & Head \\
\hline 1 & Bad Zwischenahn & $\begin{array}{l}\text { Karl-Jaspers-Hospital, Psychiatric Federation } \\
\text { Oldenburger Land }\end{array}$ & $\begin{array}{l}\text { Marianne Becker-Emner } \\
\text { Dunja Hinze-Selch }\end{array}$ \\
\hline 2 & Bonn & $\begin{array}{l}\text { Department of Psychiatry and Psychotherapy, } \\
\text { University Medical Center of Bonn }\end{array}$ & Wolfgang Maier \\
\hline 3 & Eltville-Eichberg & Vitos Hospital of Forensic Psychiatry Eltville & Roland Freese \\
\hline \multirow[t]{2}{*}{4} & \multirow[t]{2}{*}{ Fulda } & \multirow{2}{*}{$\begin{array}{l}\text { Department of Psychiatry and Psychotherapy, } \\
\text { Hospital Fulda }\end{array}$} & Adelheid Czernik \\
\hline & & & Georg Wiedemann \\
\hline 5 & Giessen & $\begin{array}{l}\text { Vitos Hospital of Forensic Psychiatry Haina, } \\
\text { Giessen }\end{array}$ & Rüdiger Müller-Isberner \\
\hline \multirow[t]{2}{*}{6} & \multirow[t]{2}{*}{ Göttingen } & \multirow{2}{*}{$\begin{array}{l}\text { Department of Psychiatry and Psychotherapy, } \\
\text { University Medical Center of Göttingen }\end{array}$} & Peter Falkai \\
\hline & & & Eckart Rüther \\
\hline 7 & Günzburg & $\begin{array}{l}\text { Department of Psychiatry and Psychotherapy, } \\
\text { District Hospital Günzburg }\end{array}$ & Thomas Becker \\
\hline 8 & Hofgeismar & $\begin{array}{l}\text { Vitos Hospital of Psychiatry and Psychotherapy } \\
\text { Merxhausen, Hofgeismar }\end{array}$ & Andreas Mielke \\
\hline 9 & Ingolstadt & $\begin{array}{l}\text { Department of Psychiatry and Psychotherapy, } \\
\text { Hospital Ingolstadt }\end{array}$ & Thomas Pollmächer \\
\hline $\mathbf{1 0}$ & Kassel & $\begin{array}{l}\text { Vitos Hospital of Psychiatry and Psychotherapy } \\
\text { Merxhausen, Kassel }\end{array}$ & Rolf Günther \\
\hline 11 & Kiel & $\begin{array}{l}\text { Hospital of Psychiatry and Psychotherapy, Center } \\
\text { for Integrative Psychiatry }\end{array}$ & Josef B. Aldenhoff \\
\hline 12 & Langenhagen & $\begin{array}{l}\text { Hospital of Psychiatry and Psychotherapy } \\
\text { Langenhagen, Regional Hospitals Hanover }\end{array}$ & Gunther Kruse \\
\hline 13 & Liebenburg & Dr. K. Fontheim's Hospital for Mental Health & Frank-Gerald Pajonk \\
\hline 14 & Lübbecke & $\begin{array}{l}\text { Department of Psychiatry and Psychotherapy, } \\
\text { Hospital Lübbecke }\end{array}$ & Udo Schneider \\
\hline \multirow[t]{2}{*}{15} & \multirow{2}{*}{$\begin{array}{l}\text { Merxhausen-Bad } \\
\text { Emstal }\end{array}$} & \multirow{2}{*}{$\begin{array}{l}\text { Vitos Hospital of Psychiatry and Psychotherapy } \\
\text { Merxhausen, Bad Emstal }\end{array}$} & Heinrich Kunze \\
\hline & & & Michael Franz \\
\hline \multirow[t]{2}{*}{16} & \multirow[t]{2}{*}{ Moringen } & \multirow[t]{2}{*}{ Hospital of Forensic Psychiatry } & Martin Schott \\
\hline & & & Dirk Hesse \\
\hline 17 & Mühlhausen & $\begin{array}{l}\text { Department of Psychiatry and Psychotherapy, } \\
\text { Ecumenical Hospital Hainich }\end{array}$ & Lothar Adler \\
\hline \multirow[t]{2}{*}{18} & \multirow[t]{2}{*}{ Rickling } & \multirow[t]{2}{*}{ Hospital of Psychiatry and Psychotherapy } & Hans-J. Schwarz \\
\hline & & & Wolfram Schreiber \\
\hline 19 & Rieden & Addiction Hospital "Am Waldsee" & Frank Löhrer \\
\hline 20 & Rostock & $\begin{array}{l}\text { Department of Psychiatry and Psychotherapy, } \\
\text { University of Rostock }\end{array}$ & Sabine Herpertz \\
\hline 21 & Taufkirchen & $\begin{array}{l}\text { Department of Psychiatry and Psychotherapy, } \\
\text { Isar-Amper-Hospital, Taufkirchen (Vils) }\end{array}$ & Matthias Dose \\
\hline 22 & Wilhelmshaven & $\begin{array}{l}\text { Department of Psychiatry and Psychotherapy, } \\
\text { Reinhard-Nieter Hospital }\end{array}$ & Here Folkerts \\
\hline \multirow[t]{2}{*}{23} & \multirow[t]{2}{*}{ Wunstorf } & \multirow{2}{*}{$\begin{array}{l}\text { Department of Psychiatry and Psychotherapy, } \\
\text { Regional Hospitals Hanover }\end{array}$} & Andreas Spengler \\
\hline & & & Cornelia Oestereich \\
\hline
\end{tabular}

\begin{tabular}{l|l|l|l}
$\mathbf{6}$ & Göttingen & Department of Transfusion Medicine & Joachim Riggert
\end{tabular}




\section{eMethods}

\section{I) GRAS Data Collection-Overview}

\section{Ethics Proposal and Internal Review Boards}

The GRAS (Göttingen Research Association for Schizophrenia) study was approved by the Ethics Committee of the Georg-August-University, Göttingen, and the local internal review boards of the collaborating centers. The research study complied with the Helsinki declaration. ${ }^{1}$

\section{Subject Demographics and Enrollment}

Subjects were included that carried the diagnosis schizophrenia according to the DSM-IV., ${ }^{2,3}$ All types (eg, paranoid, disorganized, catatonic, and undifferentiated ${ }^{4}$ ) and stages (eg, acute, chronic, residual, or remitted) were included. Also, patients with schizoaffective disorders were included. Subjects had to be at least 18 years old; there was no upper age limit. Patients were preselected by the organizers at the collaborating centers according to the diagnosis schizophrenia or schizoaffective disorder based on $D S M-I V^{2,3}$ and introduced to the traveling investigators. Every patient gave written informed consent after extensive explanation of the protocol. Of the 1071 subjects referred to the field study team, 73.9\% carried the diagnosis schizophrenia $(\mathrm{n}=792)$, $14.8 \%$ schizoaffective disorder $(\mathrm{n}=159)$, and $11.2 \%$ were suspected to have had a schizophrenic psychosis (other psychotic disorder or yet to be confirmed $[\mathrm{n}=120]$ ). The average age was $39.62 \pm$ 12.76 years, with a range from 18 to 83 years. Men $(n=714[66.7 \%])$ were $37.57 \pm 12.00$; women $(\mathrm{n}=357[33.3 \%]), 43.74 \pm 13.26$ years old.

Control subjects were voluntary blood donors, recruited by the Department of Transfusion Medicine at the Georg-August-University of Göttingen according to national guidelines for blood donation. As such, they widely fulfill health criteria, ensured by a predonation screening process containing standardized questionnaires, interviews, hemoglobin, blood pressure, pulse, and body temperature determinations. This procedure cannot entirely exclude some selection bias. For example, individuals with weight $<50 \mathrm{~kg}$ or hemoglobin $<12.5 \mathrm{~g} / \mathrm{dL}$ are excluded from blood donation. Of the 1079 subjects, $58.9 \%$ were male $(n=635)$ and $41.1 \%$ female $(n=444)$. The average age was 34.69 \pm 12.33 years, with a range from 18 to 69 years. All donors gave written informed consent. 


\section{Structured Interviews}

Structured interviews were carried out to explore school and professional education, current and employment history, partnerships and marital status, leisure activity and social network in pasttime activities, forensic history, home living situation, quality of life, migration background, medication (drug, type of neuroleptic, dosage, chlorpromazine equivalent), and history of previous physical diseases. Psychiatric comorbidities (addictions, anxiety and compulsions, depressive and manic symptoms) and symptoms related to schizophrenia such as psychotic symptoms, lack of drive, affect and thought content, disorganized behavior, speech, and parathymia were assessed employing the SCID (Structured Clinical Interview for DSM-IV). ${ }^{3}$ Efforts were undertaken to estimate onset of disease and prodrome of schizophrenia, as well as number of psychotic episodes and number and duration of hospitalizations. Special care was devoted to explore suicidality and self-harming behavior, psychotrauma, such as loss of parents or close relatives, victim status of physical abuse, disasters, or crime, neurotrauma and infections, prenatal and perinatal complications, and family history of psychiatric and physical diseases. The interviews were complemented by information obtained from medical records/charts provided by the collaborating centers.

\section{Psychometric Rating Scales}

a) PANSS (Positive and Negative Syndrome Scale) ${ }^{5}$

b) BSI (German version of Brief Symptom Inventory $)^{6}$

c) STAI (German version of State-Trait Anxiety Inventory) ${ }^{7}$

d) TAS-26 (German version of Toronto Alexithymia Scale $)^{8}$

e) CGI (Clinical Global Impressions Scale) ${ }^{9}$

f) GAF (Global Assessment of Functioning Scale) ${ }^{2}$

\section{Neuropsychological Tests}

a) Executive functioning (Trail-Making Test B) $)^{10,11}$

b) Reasoning (Subtest 3 of LPS) ${ }^{12}$

c) Verbal learning and memory (VLMT) $)^{13}$

d) Premorbid intelligence (Mehrfachwahl-Wortschatz Test B [MWT-B] $)^{14}$

e) Working memory (Letter-number sequencing test, subtest of WAIS-III) ${ }^{15}$

f) Processing speed (Trail-Making Test A) $)^{10,11}$

g) Psychomotor speed, concentration, and attention (digit symbol test, subtest of HAWIE-R) ${ }^{16}$

h) Verbal fluency (category and letters) ${ }^{17,18}$ 
i) Fine motor function, speed, and coordination (tapping and dotting) ${ }^{19}$

j) Alertness and divided attention (TAP) ${ }^{20}$

\section{Neurological Tests}

a) Cambridge Neurological Inventory $(\mathrm{CNI})^{21}$

b) Contralateral Co-Movement Task (COMO) ${ }^{22}$

c) Olfactory testing (commercially available olfactory labels [www.sensonics.com] were used for odor identification in a multiple choice setting or free naming and assignment of odor properties/odor interpretation)

d) Barnes-Akathisia Scale (BAS) ${ }^{23}$

e) Simpson-Angus Scale (SAS) ${ }^{24}$

f) Abnormal Involuntary Movement Scale (AIMS) ${ }^{9}$

g) Tardive Dyskinesia Rating Scale (TDRS) ${ }^{25}$

\section{II) Genetic Analyses}

\section{DNA Extraction and Normalization}

Genomic DNA was purified from whole blood using JETQUICK Blood \& Cell Culture DNA Spin Kit (Genomed GmbH, Löhne, Germany) according to the manufacturer's protocol. Resulting DNA samples were divided into aliquots and stored at $-80^{\circ} \mathrm{C}$. For further analysis, DNA was normalized to $50 \mathrm{ng} / \mu \mathrm{L}$ with an automated robotic platform (Microlab Star; Hamilton, Bonaduz, Switzerland). For quality control, each sample was analyzed with a $0.8 \%$ agarose gel.

\section{Analysis of the Coding Region of CPLX2}

Polymerase Chain Reaction (PCR): The coding region of CPLX2 was amplified via 2 PCR reactions. Primers were synthesized using a 3900 DNA Synthesizer (Applied Biosystems, Foster City, California). The first fragment (exons 4 and 5) was amplified with the following primers:

CPLX2_Ex4_5 forward: 5'-AAATAGGGACCAAACCGCTTTCA-3'

CPLX2_Ex4_5 reverse: 5'-GGTCCCAAAGATCTGCAGTACGA-3'.

The second PCR (exon 6) was carried out with the primers:

CPLX2_Ex6 forward: 5'-CCATTGGTTCAAGTCAGATTCGTC-3'

CPLX2_Ex6 reverse: 5'-GTCACCACACTAGCACGACCTTAG-3'. 
For each sample, the reaction mixture $(21 \mu \mathrm{L})$ was prepared in 384-well plates, each containing 100 ng of human genomic DNA, $\mathrm{NH}_{4}$ buffer $(1 \times), 120 \mu \mathrm{M}$ dNTPs each, $2.4 \mathrm{mM} \mathrm{MgCl}_{2}, 200 \mathrm{nM}$ forward and reverse primers, and $1 \mathrm{U}$ Diamond polymerase (Bioline, Luckenwalde, Germany). The cycling program was carried out after a preheating step at $94^{\circ} \mathrm{C}$ for $5 \mathrm{~min}$ and included 30 cycles of (1) denaturation at $94^{\circ} \mathrm{C}$ for $30 \mathrm{~s},(2)$ annealing at $60^{\circ} \mathrm{C}$ for $30 \mathrm{~s}$, and (3) extension at $72^{\circ} \mathrm{C}$ for $60 \mathrm{~s}$ in a DNA thermal cycler (PTC-200 MJ Research; BioRad, Munich, Germany).

Sequencing: The PCR amplicons were purified from unincorporated primers and dNTPs by digesting with $1 \mathrm{U}$ shrimp alkaline phosphatase (SAP) und $5 \mathrm{U}$ Exonuclease I (Exo) according to the manufacturer's instructions (USB Europe GmbH, Staufen, Germany). For analysis on the ABI 3730 XL DNA analyzer sequencing reactions $(12 \mu \mathrm{L})$ were prepared in 384-well plates, each containing $4 \mu \mathrm{L}$ purified PCR product, sequencing buffer $(\times 1)$ and $300 \mathrm{nM}$ primer (same as used for PCR; sense or antisense) using the BigDye Terminator v3.1 Cycle Sequencing kit (Applied Biosystems). The following PCR thermal cycling profile was used after a preheating step at $94^{\circ} \mathrm{C}$ for $30 \mathrm{~s}$ : 25 cycles of (1) $96^{\circ} \mathrm{C}$ for $10 \mathrm{~s}$; (2) $50^{\circ} \mathrm{C}$ for $10 \mathrm{~s}$; (3) $60^{\circ} \mathrm{C}$ for $2 \mathrm{~min}$. When cycling was complete, the sequencing reactions were precipitated using ethanol and magnetic beads (AgenCourt Clean Seq; GC Biotech, Alphen aan den Rijn, Netherlands) to eliminate unincorporated fluorescentlabeled nucleotides. Raw data were processed with sequencing analysis 5.2 (Applied Biosystems) and with different modules of the software package Lasergene 7.0 (DNASTAR, Inc, Madison, Wisconsin).

\section{Analysis of 5' Untranslated Region (UTR) of CPLX2}

Selecting Single-Nucleotide Polymorphisms (SNPs) in 5' region of CPLX2: For SNP selection, a list of potential tag SNPs was created using Haploview ${ }^{26}$ (http://www.broad.mit.edu/haploview /haploview/) with the genomic region Chr5:175,149,893..175,243,629. Out of these tag SNPs, several SNPs with minor allele frequency (MAF) of at least $>0.3$ were selected to cover the $5^{\prime}$ region of CPLX2. The SNPs rs6868608, rs2443541, and rs1366116 lie in putative promoter regions of the 2 different transcripts. The marker rs2243404 is located in the noncoding exon 1, and rs4242187, rs10072860, and rs4868539 cover the rest of the region.

Genotyping: The selected SNPs were analyzed using Simple Probes (TIB Molbiol, Berlin, Germany) and called using the LightCycler 480 Genotyping Software implemented in the LightCycler 
480 system (Roche, Mannheim, Germany). The reaction mixture (10 $\mu \mathrm{L})$ was prepared with $40 \mathrm{ng}$ of DNA in 384-well plates according to standard protocols (Roche). The concentration of $\mathrm{MgCl}_{2}$ and the Genotyping Master Mix were adapted for each assay. The cycle conditions were as follows: denaturation of the template DNA with $95^{\circ} \mathrm{C}$ for $10 \mathrm{~min}$, amplification of the target DNA for 45 cycles of (1) $95^{\circ} \mathrm{C}$ for $10 \mathrm{~s},(2) 60^{\circ} \mathrm{C}$ for $10 \mathrm{~s}$, and $(3) 72^{\circ} \mathrm{C}$ for $15 \mathrm{~s}$ (temperature ramp rate $4.6^{\circ} \mathrm{C} / \mathrm{s}$ in steps 1 and 3 and $2.4^{\circ} \mathrm{C}$ in 2). Melting curve analysis was performed with $30 \mathrm{~s}$ of $95^{\circ} \mathrm{C}, 40^{\circ} \mathrm{C}$ for $2 \mathrm{~min}$ (ramp rate $2.0^{\circ} \mathrm{C} / \mathrm{s}$ ) and then a continuous ramping to $75^{\circ} \mathrm{C}$ with 3 acquisitions per ${ }^{\circ} \mathrm{C}$. The temperatures for the melting peaks in each assay are listed in eTable 1. In each run, 8 positive controls (hgDNA; Bioline) and negative water blanks were included for quality and internal control purposes. Overall, successfully genotyped markers amounted to $99.6 \%$ to $99.7 \%$.

\section{Micro-RNA Analysis}

Human CPLX2 3'UTR Luciferase: The first 274 bp of the 3'UTR of CPLX2, containing SNP rs3822674 with either $\mathrm{C}$ or $\mathrm{T}$ allele, were PCR amplified from respective human samples. The following primers were used:

hCPLX2_3'UTR forward: 5'-GCGTCTAGACCAGGCCTCCTGCCCCAGC-3' hCPLX2_3'UTR reverse: 5'-CGCGCGGCCGCACGACCTTAGGCTTCCTGAGGGG-3'.

The cloning into the Renilla luciferase vector phRL-SV40 (Promega, Mannheim, Germany) was performed using XbaI and NotI restriction enzyme sites. The resulting constructs (phRL-C CPLX23'UTR and phRL-T CPLX2-3'UTR) were verified by sequencing.

Luciferase Assays: Neuro2a (N2a) cells (LGC Standards GmbH, Wesel, Germany) were plated in 96-well cell culture plates (NUNC, Langenselbold, Germany) at 15000 cells per well in DMEM supplemented with 5\% FCS without antibiotics. At 16 to $18 \mathrm{~h}$ after plating, cells were transfected with Lipofectamine 2000 (Invitrogen, Karlsruhe, Germany) using the manufacturer's protocol. In each well, 1 ng of phRL-SV40 (vector without 3'UTR, C or T construct) and 1 pg of pCMV-FFluccontrol vector (Promega) were cotransfected with/without 1 pmol of hsa-miR-498 (Ambion, Foster City, California). For each treatment, 6 replicates were performed. The dual-luciferase reporter assay (Promega) was used according to the manufacturer's protocol. At $24 \mathrm{~h}$ after transfection, cells in each well were lysed using $30 \mu \mathrm{L}$ Passive Lysis Buffer (Promega). The plates were incubated for $15 \mathrm{~min}$ at room temperature with slight shaking $(200 \mathrm{rpm})$. Lysates were assayed immediately or 
frozen at $-20^{\circ} \mathrm{C}$. Prior to measurement, lysates were transferred into a black plastic microtiter plate. Measurements were performed with the microplate reader Mitras LB940 (Berthold Technologies, Regensdorf, Switzerland) and associated software MicroWin 2000. Renilla values were divided by the corresponding firefly readings producing values expressed as relative luciferase units (RLU).

\section{Analysis of Peripheral Blood Mononuclear Cells (PBMCs)}

PBMCs of schizophrenic patients with 1 of the 3 different genotypes (CC, CT, or TT) at the SNP rs3822674 in the 3' UTR were isolated using the standard Ficoll-Paque Plus isolation procedure (GE Healthcare, Munich, Germany). For RNA isolation the miRNeasy Mini Kit (Qiagen, Hilden, Germany) was used. A total of $1 \mu \mathrm{g}$ RNA, a mixture of oligo dT and hexamer primers and dNTPS (10mM each) were used for the transcription into cDNA using $200 \mathrm{U}$ SuperScriptIII (Invitrogen) in a $20-\mu \mathrm{L}$ reaction. The mixture was incubated for $10 \mathrm{~min}$ at $25^{\circ} \mathrm{C}, 45 \mathrm{~min}$ at $50^{\circ} \mathrm{C}$, followed by 45 $\min$ at $55^{\circ} \mathrm{C}$.

For the quantitative reverse transcription (qRT)PCR, the cDNA was used 1:10 diluted and 4 replicates per sample were performed; $5 \mu \mathrm{L}$ Power SYBR mix (Applied Biosystems) and 1 pmol of each primer (see below) were added. The following cycling profile was used for the LightCycler 480 system (Roche): preheating step at $95^{\circ} \mathrm{C}$ for $10 \mathrm{~min}$; 45 cycles of (1) $95^{\circ} \mathrm{C}$ for $15 \mathrm{~s},(2) 60^{\circ} \mathrm{C}$ for 1 min. Cycle threshold (CT) values for CPLX2 were standardized to CT values of GAPDH.

hCPLX2_qRT-PCR forward: 5'-TTTGGTGAGAAGCCAATTCC-3'

hCPLX2_qRT-PCR reverse: 5'-CATCCCCACACAACTGACTG-3'

hGAPDH_qRT-PCR forward: 5'-CTGACTTCAACAGCGACACC-3'

hGAPDH_qRT-PCR reverse: 5'-TGCTGTAGCCAAATTCGTTGT-3'.

\section{III) Statistical Analyses}

To evaluate the experimental data (animal study, expression experiments), GraphPad PRISM version 5.01 for windows was employed. For the expression studies, Mann-Whitney $U$ test, 2 tailed, was applied; for the animal data, 2-way analysis of variance for repeated measures was used. Statistical analyses of the SNPs studied either by sequencing or by direct genotyping were performed using SPSS for windows version 17.0 (https://www.spss.com/de) and R2.8.1 (http://cran.r-project.org). Correlations of the 3 neuropsychological target variables (executive 
functioning, reasoning, and verbal learning/memory) and the constitutive variable (premorbid intelligence) were assessed using Pearson product-moment correlation. ${ }^{27}$ Cronbach $\alpha$ coefficient ${ }^{28}$ was determined for estimation of internal consistency of the 3 target variables.

All metric phenotypic variables were standardized by Blom Transformation ${ }^{29}$ prior to statistical analysis. The latter is a probit transformation of the ranks $r_{i}$ obtained on all $n$ nonmissing values of a selected phenotype,

$y_{i}=\Phi^{-1}\left(\left(r_{i}-3 / 8\right) /(n+1 / 4)\right)$

where $\Phi^{-1}$ is the quantile function of the standard normal distribution. The resulting standardized phenotypes are normally distributed with zero mean and variance one. Analysis of covariance was employed to compare means of metric phenotypic variables for genotypes at each marker, assuming a codominant model and adjusting for covariates sex and age, in case of premorbid intelligence also for non-German speakers with language problems $(8.6 \%$ of the total sample; $1.9 \%$ had to be taken out completely due to severe language difficulties). To adjust for multiple testing, a permutation procedure was carried out as follows: Let $K$ denote the observed number of $P$ values $<.05$ for a trait or for a set of $m$ traits on the 11 CPLX2 markers. The multiple-testing-adjusted $P$ value is the probability to obtain at least $K P$ values $<.05$ on the respective set of tests by chance under the null hypothesis of no genotype-phenotype association. The latter was simulated by generating $\mathrm{n}=10000$ replications of the original sample, each with permuted assignment of the individual phenotype (consisting of sex, age, and cognitive trait values). This conserves the underlying genomic/genotype structure for each individual. The program Haploview version $4.1^{26}$ was used to evaluate the CPLX2 markers (test of Hardy-Weinberg equilibrium for each marker, calculation of D' between SNP pairs, and identification of linkage disequilibrium [LD] blocks using the Solid Spine of LD method).

Haplotype analyses were performed using UNPHASED v3.0.13, ${ }^{30}$ PLINK v1.06, ${ }^{31}$ and SAS v9.1. ${ }^{32}$ UNPHASED was used for haplotype association analyses of binary categorical variables, carrying out likelihood ratio tests in a log-linear model through unconditional logistic regression adjusting for age and sex. $P$ values provided in the manuscript for the case-control study are nominal. The genomic analysis package PLINK was used for the estimation and tabulation of all possible individuals' haplotype phases and the corresponding posterior probabilities (ie, given genotype data) for posterior analyses of quantitative traits in SAS. The latter employs a linear model on the Blom-transformed cognitive trait values, allowing for ambiguous haplotypes by weighted regression 
with individual haplotype probabilities as weights. The model includes variables for all haplotypes at a selected location (all sample frequencies were $>1 \%$ ), adjusts for covariates sex, age, and for marker genotype at other associated CPLX2 regions (being in low LD with the considered haplotype gene region). For premorbid intelligence estimates, the data had to be additionally adjusted for nonnative German speakers with language problems (8.6\% of our sample). Due to severe language problems, $1.9 \%$ of the sample had to be excluded. The trait mean given an individual's genotype data is based on an additive model of haplotypes assuming a normal trait distribution.

\section{IV) Database Sequence Analyses of CPLX2 Gene}

To explore how a single base pair substitution might influence expression of CPLX2 and therefore cognition, several sequence analyses were performed. In order to reject the existence of other genes in the analyzed region, BLAST $\operatorname{search}^{33}$ was performed (http://blast.ncbi.nlm.nih.gov/Blast.cgi) using our sequence and its reverse complement. Furthermore, sequence similarities regarding microRNAs were ascertained with the program miRAlign ${ }^{34}$ (http://bioinfo.au.tsinghua.edu.cn /miralign) and the existence of known microRNAs in the region of interest was also checked by searching the microRNA database of the Sanger Institute (http://microrna.sanger.ac.uk/cgi-bin /sequences). Additionally, putative target sites for known microRNAs were analyzed with TargetScan ${ }^{35}$ (http://www.targetscan.org/). A few of these predicted binding sites were in the region of rs3822674. Therefore, we performed a $\Delta \Delta \mathrm{G}$ prediction analysis for both alleles of rs 3822674 and different microRNAs (http://genie.weizmann.ac.il/pubs/mir07/mir07_prediction.html). The analysis was performed with the first 274 bp of the 3'UTR of CPLX2. Conservation status in CPLX2, and specifically in the regions of the SNPs, was evaluated by aligning the sequences of the exons, introns, the region around the SNPs ( $\pm 200 \mathrm{bp})$ and the single SNP position (eFigure 2 and eTable 8). For this purpose, we used the 28 species comparison method based on Siepel et al, 2005, ${ }^{36}$ implemented in the UCSC (http://genome.ucsc.edu) Web site.

\section{V) Behavioral Studies in Mice}

Animals: The gene targeting strategy for the Cplx2-null mutant mice was described previously. ${ }^{28}$ Behavioral experiments were performed after more than 10 backcrosses to the C57BL/6NCrl (Charles River Laboratories, Sulzfeld, Germany) mouse strain, and employed age-matched male WT $(\mathrm{n}=25)$ and Cplx2-null mutant $(\mathrm{n}=35)$ littermates. Mice were housed at 4 to 5 per cage in a 
room with a 12-h light-dark cycle (lights on at 09:00 h) with ad libitum access to food and water. The age of mice at the beginning of testing was 10 months. Behavioral tests were conducted in a blind fashion during the light phase of the day from 10:00 until 17:00 h. All experiments were performed with permission of the Bezirksregierung Braunschweig (Local Animal Care and Use Committee) in accordance with the German Animal Protection Law.

Juvenile Parietal Cryolesion $^{29}$ : At murine puberty (age 28 days), male mice were anesthetized with intraperitoneal (IP) injection of $0.25 \%$ tribromoethanol (Avertin; SIGMA-Aldrich, Taufkirchen, Germany) (0.125 mg/g). The parietal skull was exposed through scalp incision and a freezing lesion was placed through the intact skull on the right parietal cortex (coordinates from bregma: $1.5 \mathrm{~mm}$ posterior, $1.5 \mathrm{~mm}$ lateral). A cone-shaped copper cylinder with tip diameter of $1 \mathrm{~mm}$ was cooled with liquid nitrogen $\left(-183^{\circ} \mathrm{C}\right)$. Using a stereotactic device, the tip was placed in direct contact with the exposed parietal skull and kept in place for $60 \mathrm{~s}$. Sham-operated animals went through the same procedure without cooled metal cone.

Morris Water Maze: Spatial learning and memory was assessed in a water maze as described previously. ${ }^{37,38}$ A large circular tank (diameter, $1.2 \mathrm{~m}$; depth, $0.4 \mathrm{~m}$ ) was filled with opaque water $\left(25^{\circ} \pm 1^{\circ} \mathrm{C}\right.$, depth, $\left.0.3 \mathrm{~m}\right)$ and the escape platform $(10 \mathrm{~cm} \times 10 \mathrm{~cm})$ submerged $1 \mathrm{~cm}$ below the surface. Mouse swim patterns were monitored by a computer and video-tracking system Viewer 2 (Biobserve GmbH, Bonn, Germany). Escape latency, swim speed, path length, and trajectory of swimming were recorded for each mouse. During the first 2 days, mice were trained to swim to a visible platform (visible platform task) marked with a 15-cm-high black flag and placed pseudorandomly in different locations across trials (nonspatial training). The extra-maze cues were hidden during these trials. After 2 days of visible platform training, hidden platform training (spatial training) was performed. For 8 days, mice were trained to find a hidden platform (ie, the flag was removed) that was located at the center of 1 of the 4 quadrants of the pool. The location of the platform was fixed throughout testing. Mice had to navigate using extra-maze cues that were located on the walls of the testing room. Every day, mice went through 4 trials with an intertrial interval of $5 \mathrm{~min}$. The mice were placed into the pool facing the side wall randomly at 1 of 4 start locations and allowed to swim until they found the platform, or for a maximum of $90 \mathrm{~s}$. Any mouse that failed to find the platform within $90 \mathrm{~s}$ was gently guided to the platform. The animal then remained on the platform for $20 \mathrm{~s}$ before being removed from the pool. The next day after completion of the hidden platform training, a probe trial was conducted in order to determine 
whether mice used a spatial strategy to find the platform or not. For that purpose, the platform was removed from the pool and mice were allowed to swim freely for $90 \mathrm{~s}$. The percentage of time spent in each quadrant of the pool as well as the number of times the mice crossed the former position of the hidden platform were recorded. 
eTable 1. Allele-Specific Melting Temperatures for Simple Probe Assays

\begin{tabular}{|c|c|c|}
\hline & \multicolumn{2}{|c|}{ Alleles } \\
\hline rs6868608 & A & T \\
\hline Melting ${ }^{\circ} \mathrm{C}$ & 53 & 49 \\
\hline & & $\mathrm{T}$ \\
\hline rs2443541 & $\mathrm{C}$ & 54 \\
\hline Melting ${ }^{\circ} \mathrm{C}$ & 62 & $\mathrm{~T}$ \\
\hline & $\mathrm{C}$ & 58 \\
\hline rs2243404 & 66 & $\mathrm{~T}$ \\
\hline Melting ${ }^{\circ} \mathrm{C}$ & & 55 \\
\hline & $\mathrm{C}$ & $\mathrm{T}$ \\
\hline rs4242187 & 63 & 55 \\
\hline Melting ${ }^{\circ} \mathrm{C}$ & & $\mathrm{G}$ \\
\hline & 61 & $\mathrm{G}$ \\
\hline rs10072860 & & 58 \\
\hline Melting ${ }^{\circ} \mathrm{C}$ & $\mathrm{A}$ & $\mathrm{T}$ \\
\hline & 65 & 62 \\
\hline rs4868539 & & \\
\hline Melting ${ }^{\circ} \mathrm{C}$ & $\mathrm{C}$ & \\
\hline & 55 & \\
\hline rs1366116 & & \\
\hline Melting ${ }^{\circ} \mathrm{C}$ & & \\
\hline
\end{tabular}


eTable 2. Characteristics of Analyzed SNPs ${ }^{a}$

\begin{tabular}{|c|c|c|c|}
\hline Marker & Name & MAF & Position \\
\hline 1 & rs6868608 & T: 0.360 & $175,150,010$ \\
\hline 2 & rs2443541 & T: 0.317 & $175,154,594$ \\
\hline 3 & rs2243404 & T: 0.317 & $175,156,222$ \\
\hline 4 & rs4242187 & T: 0.467 & $175,176,703$ \\
\hline 5 & rs10072860 & T: 0.457 & $175,195,299$ \\
\hline 6 & rs4868539 & A: 0.458 & $175,212,047$ \\
\hline 7 & rs1366116 & C: 0.442 & $175,230,137$ \\
\hline 8 & rs3892909 & C: 0.389 & $175,238,197$ \\
\hline 9 & rs3822674 & C: 0.467 & $175,239,862$ \\
\hline 10 & rs56934064 & - & $175,239,899$ \\
\hline 11 & $+251 E x 6^{\text {a }}$ & - & $175,239,905$ \\
\hline
\end{tabular}

Abbreviations: MAF, minor allele frequency; SNP, single-nucleotide polymorphism.

${ }^{a}$ Displayed are the analyzed markers with their official symbols, their minor allele frequencies according to NCBI, and their exact position on chromosome 5.

${ }^{\mathrm{b}}$ Marker $11(+251$ Ex6) is a novel SNP. 
eTable 3. Point Mutations Detected in the Sequenced Region of CPLX2

\begin{tabular}{|c|c|c|c|c|}
\hline & No. of $\mathrm{SCZ} / \mathrm{CON}$ & Substitution & Mutation Type & Position \\
\hline \multicolumn{5}{|c|}{ Coding sequence } \\
\hline 168 Ex6 & $1 \times \mathrm{SCZ}^{\mathrm{a}}$ & $\mathrm{G}$ to $\mathrm{G} / \mathrm{A}$ & $\begin{array}{c}\text { Missense } \\
\text { (Gly to Arg) }\end{array}$ & $175,239,625$ \\
\hline $72 \mathrm{Ex} 5$ & $1 \times \mathrm{SCZ}$ & $\mathrm{G}$ to $\mathrm{G} / \mathrm{A}$ & Silent & $175,238,589$ \\
\hline 94Ex4 & $1 \times \mathrm{SCZ}$ & $\mathrm{C}$ to $\mathrm{C} / \mathrm{A}$ & Silent & $175,238,609$ \\
\hline $130 \mathrm{Ex} 5$ & $1 \times \mathrm{CON}$ & $\mathrm{C}$ to $\mathrm{C} / \mathrm{T}$ & $\begin{array}{c}\text { Missense } \\
\text { (Arg to Cys) }\end{array}$ & $175,238,645$ \\
\hline \multicolumn{5}{|c|}{ Noncoding sequence } \\
\hline$-6 \mathrm{Ex} 4$ & $1 \times \mathrm{SCZ}$ & $\mathrm{G}$ to $\mathrm{G} / \mathrm{A}$ & - & $175,238,257$ \\
\hline$+20 \mathrm{Ex} 4$ & $1 \times \mathrm{SCZ}$ & $\mathrm{C}$ to $\mathrm{C} / \mathrm{G}$ & - & $175,238,398$ \\
\hline$+21 \mathrm{Ex} 5^{\mathrm{b}}$ & $3 \times \mathrm{SCZ}$ & $\mathrm{C}$ to $\mathrm{C} / \mathrm{G}$ & - & $175,238,713$ \\
\hline-116 Ex6 & $1 \times \mathrm{SCZ}$ & $\mathrm{G}$ to $\mathrm{G} / \mathrm{A}$ & - & $175,239,341$ \\
\hline-42 Ex6 & $1 \times \mathrm{SCZ}$ & $\mathrm{C}$ to $\mathrm{C} / \mathrm{T}$ & - & $175,239,415$ \\
\hline \multirow{2}{*}{+31 Ex6 } & $1 \times \mathrm{SCZ}$ & \multirow{2}{*}{$\mathrm{G}$ to $\mathrm{G} / \mathrm{A}$} & \multirow{2}{*}{-} & \multirow{2}{*}{$175,239,760$} \\
\hline & $1 \times \mathrm{CON}$ & & & \\
\hline+84 Ex6 & $1 \times \mathrm{CON}$ & $\mathrm{T}$ to $\mathrm{T} / \mathrm{A}$ & - & $175,239,814$ \\
\hline+104 Ex6 & $1 \times \mathrm{CON}$ & $\mathrm{T}$ to $\mathrm{T} / \mathrm{C}$ & - & $175,239,833$ \\
\hline
\end{tabular}

Abbreviations: CON, control subjects; SCZ, schizophrenic subjects.

${ }^{a}$ Healthy father of the affected patient is also carrier of this missense mutation.

$\mathrm{b}_{+} 21$ Ex5 is known as single-nucleotide polymorphism rs4077873; in our population the frequency is $<1 \%$. 
eTable 4. Frequencies of Single SNP Analysis (Case-Control) ${ }^{\mathrm{a}}$

\begin{tabular}{|c|c|c|c|c|c|c|c|c|}
\hline \multirow{2}{*}{\multicolumn{2}{|c|}{ SNP }} & \multicolumn{3}{|c|}{$\begin{array}{c}\text { Genotypic Frequencies, } \\
\%\end{array}$} & \multirow{2}{*}{$\begin{array}{c}\chi^{2 \mathrm{~b}} \\
\text { (P Value) }\end{array}$} & \multicolumn{2}{|c|}{$\begin{array}{c}\text { Allelic } \\
\text { Frequencies, \% }\end{array}$} & \multirow{4}{*}{$\begin{array}{c}\begin{array}{c}\chi^{2 \mathrm{c}} \\
\text { (P Value) }\end{array} \\
0.35 \\
(.55)\end{array}$} \\
\hline & & $\mathbf{A A}$ & AT & TT & & A & $\mathbf{T}$ & \\
\hline \multirow{2}{*}{ rs6868608 } & SCZ & 35.1 & 46.0 & 18.9 & \multirow{2}{*}{$\begin{array}{l}3.58 \\
(.17) \\
\end{array}$} & 58.1 & 41.9 & \\
\hline & $\mathrm{CON}$ & 34.2 & 49.5 & 16.3 & & 59.0 & 41.0 & \\
\hline & & $\mathrm{CC}$ & CT & TT & & $\mathrm{C}$ & $\mathbf{T}$ & \\
\hline \multirow{3}{*}{ rs2443541 } & SCZ & 62.1 & 33.6 & 4.3 & \multirow{2}{*}{$\begin{array}{l}0.59 \\
(.74)\end{array}$} & 78.9 & 21.1 & \multirow{2}{*}{$\begin{array}{l}0.43 \\
(.51)\end{array}$} \\
\hline & $\mathrm{CON}$ & 60.5 & 35.1 & 4.4 & & 78.1 & 21.9 & \\
\hline & & $\mathrm{CC}$ & CT & TT & & $\mathrm{C}$ & $\mathbf{T}$ & \\
\hline \multirow{3}{*}{ rs2243404 } & SCZ & 63.8 & 32.0 & 4.2 & \multirow{2}{*}{$\begin{array}{l}0.60 \\
(.74)\end{array}$} & 79.8 & 20.2 & \multirow{2}{*}{$\begin{array}{l}0.52 \\
(.47)\end{array}$} \\
\hline & $\mathrm{CON}$ & 62.2 & 33.5 & 4.3 & & 78.9 & 21.1 & \\
\hline & & $\mathrm{CC}$ & CT & TT & & $\mathrm{C}$ & $\mathbf{T}$ & \\
\hline \multirow{3}{*}{ rs 4242187} & $\mathrm{SCZ}$ & 45.3 & 43.4 & 11.3 & \multirow{2}{*}{$\begin{array}{l}1.39 \\
(.50)\end{array}$} & 67.0 & 33.0 & \multirow{2}{*}{$\begin{array}{l}0.43 \\
(.51) \\
\end{array}$} \\
\hline & $\mathrm{CON}$ & 45.0 & 42.1 & 12.9 & & 66.0 & 34.0 & \\
\hline & & GG & GT & TT & & $\mathbf{G}$ & $\mathbf{T}$ & \\
\hline \multirow{3}{*}{ rs 10072860} & SCZ & 23.8 & 48.7 & 27.5 & \multirow{2}{*}{$\begin{array}{l}1.81 \\
(.40)\end{array}$} & 48.2 & 51.8 & \multirow{2}{*}{$\begin{array}{l}0.02 \\
(.88)\end{array}$} \\
\hline & $\mathrm{CON}$ & 22.6 & 51.6 & 25.8 & & 48.4 & 51.6 & \\
\hline & & $\mathbf{A A}$ & $\mathbf{A G}$ & GG & & A & G & \\
\hline \multirow{3}{*}{ rs4868539 } & $\mathrm{SCZ}$ & 23.3 & 47.7 & 29.0 & \multirow{2}{*}{$\begin{array}{l}0.73 \\
(.69)\end{array}$} & 47.1 & 52.9 & \multirow{2}{*}{$\begin{array}{l}0.03 \\
(.87)\end{array}$} \\
\hline & $\mathrm{CON}$ & 23.9 & 45.9 & 30.2 & & 46.9 & 53.1 & \\
\hline & & $\mathrm{CC}$ & CT & TT & & $\mathrm{C}$ & $\mathbf{T}$ & \\
\hline \multirow{3}{*}{ rs1366116 } & SCZ & 15.5 & 44.8 & 39.7 & \multirow{2}{*}{$\begin{array}{l}0.84 \\
(.66) \\
\end{array}$} & 37.9 & 62.1 & \multirow{2}{*}{$\begin{array}{l}0.19 \\
(.66) \\
\end{array}$} \\
\hline & $\mathrm{CON}$ & 15.2 & 46.8 & 38.0 & & 38.6 & 61.4 & \\
\hline & & $\mathrm{CC}$ & CT & TT & & $\mathrm{C}$ & $\mathbf{T}$ & \\
\hline \multirow{3}{*}{ rs3892909 } & SCZ & 13.6 & 43.9 & 42.5 & \multirow{2}{*}{$\begin{array}{l}0.59 \\
(.74)\end{array}$} & 35.6 & 64.4 & \multirow{2}{*}{$\begin{array}{l}0.01 \\
(.92)\end{array}$} \\
\hline & $\mathrm{CON}$ & 13.0 & 45.5 & 41.5 & & 35.7 & 64.3 & \\
\hline & & $\mathrm{CC}$ & CT & TT & & $\mathrm{C}$ & $\mathbf{T}$ & \\
\hline & SCZ & 25.6 & 49.2 & 25.2 & 0.09 & 50.2 & 49.8 & 0.02 \\
\hline rs3822674 & $\mathrm{CON}$ & 25.1 & 49.8 & 25.1 & $(.96)$ & 50.0 & 50.0 & $(.90)$ \\
\hline & & $\mathbf{A A}$ & $\mathbf{A G}$ & GG & & $\mathbf{A}$ & $\mathbf{G}$ & \\
\hline & $\mathrm{SCZ}$ & 52.7 & 39.8 & 7.5 & 1.35 & 72.6 & 27.4 & 0.00 \\
\hline rs56934064 & $\mathrm{CON}$ & 51.7 & 41.8 & 6.5 & $(.51)$ & 72.6 & 27.4 & $(.97)$ \\
\hline & & $\mathrm{CC}$ & CG & GG & & $\mathrm{C}$ & $\mathbf{G}$ & \\
\hline$+251 \mathrm{Fv} 6$ & SCZ & 7.6 & 39.4 & 53.0 & 1.72 & 27.3 & 72.7 & 0.44 \\
\hline$+251 \mathrm{Ex} 6$ & $\mathrm{CON}$ & 7.2 & 42.1 & 50.7 & $(.42)$ & 28.2 & 71.8 & $(.51)$ \\
\hline
\end{tabular}

Abbreviations: CON, control subjects; SCZ, schizophrenic subjects; SNP, single-nucleotide polymorphism.

${ }^{a}$ This table shows the case-control data for each marker. Test statistics and $P$ values both for genotypic and allelic frequencies were calculated for each marker using $\chi^{2}$ test $\left({ }^{\mathrm{b}} 2\right.$ degrees of freedom, ${ }^{\mathrm{c}} 1$ degree of freedom). There are no significant differences between cases and controls regarding genotypic or allelic frequencies. All markers fulfilled the Hardy-Weinberg equilibrium criteria. 
eTable 5. Pairwise Association Test on Cognitive Measures of Schizophrenic Patients ${ }^{\mathrm{a}}$

\begin{tabular}{|c|c|c|c|c|c|c|}
\hline & rs2443541 & rs2243404 & rs10072860 & rs1366116 & rs3892909 & rs3822674 \\
\hline Target Variables & \multicolumn{6}{|c|}{$F$ Values (P Values) } \\
\hline \multirow{3}{*}{$\begin{array}{l}\text { Combined } \\
\text { (multivariate) }\end{array}$} & & $\begin{array}{c}\text { CT/TT } \\
7.27(.007)\end{array}$ & & $\begin{array}{c}\text { CT/TT } \\
7.56(.006)\end{array}$ & $\begin{array}{c}\text { CT/TT } \\
10.06(.002)\end{array}$ & $\begin{array}{c}\mathrm{TC} / \mathrm{TT} \\
0.008(.93)\end{array}$ \\
\hline & & $\begin{array}{c}\text { CC/TT } \\
7.12(.008)\end{array}$ & & $\begin{array}{c}\mathrm{CC} / \mathrm{TT} \\
0.08(.78)\end{array}$ & $\begin{array}{c}\mathrm{CC} / \mathrm{TT} \\
1.65(.20)\end{array}$ & $\begin{array}{c}\text { CC/TT } \\
1.43(.04)\end{array}$ \\
\hline & & $\begin{array}{c}\mathrm{CC} / \mathrm{CT} \\
0.03(.86)\end{array}$ & & $\begin{array}{c}\mathrm{CC} / \mathrm{CT} \\
3.35(.07)\end{array}$ & $\begin{array}{c}\mathrm{CC} / \mathrm{CT} \\
1.07(.30)\end{array}$ & $\begin{array}{c}\mathrm{CC} / \mathrm{TC} \\
5.20(.02)\end{array}$ \\
\hline \multicolumn{7}{|l|}{ Individual (univariate) } \\
\hline \multirow{3}{*}{ Executive functioning } & $\begin{array}{c}\text { CT/TT } \\
3.89(.05)\end{array}$ & $\begin{array}{c}\text { CT/TT } \\
5.83(.02)\end{array}$ & $\begin{array}{c}\mathrm{GT} / \mathrm{TT} \\
0.33(.568)\end{array}$ & & & \\
\hline & $\begin{array}{c}\mathrm{CC} / \mathrm{TT} \\
0.68(.41)\end{array}$ & $\begin{array}{c}\mathrm{CC} / \mathrm{TT} \\
2.28(.13)\end{array}$ & $\begin{array}{c}\text { GG/TT } \\
5.83(.02)\end{array}$ & & & \\
\hline & $\begin{array}{c}\mathrm{CC} / \mathrm{CT} \\
6.98(.008)\end{array}$ & $\begin{array}{c}\mathrm{CC} / \mathrm{CT} \\
3.79(.05)\end{array}$ & $\begin{array}{c}\text { GG/GT } \\
4.86(.03)\end{array}$ & & & \\
\hline \multirow{3}{*}{ Reasoning } & & & & $\begin{array}{c}\text { CT/TT } \\
9.92(.002)\end{array}$ & $\begin{array}{c}\mathrm{CT} / \mathrm{TT} \\
9.38(.002)\end{array}$ & $\begin{array}{c}\mathrm{TC} / \mathrm{TT} \\
2.01(.16)\end{array}$ \\
\hline & & & & $\begin{array}{c}\mathrm{CC} / \mathrm{TT} \\
1.36(.25) \\
\end{array}$ & $\begin{array}{c}\mathrm{CC} / \mathrm{TT} \\
3.63(.06) \\
\end{array}$ & $\begin{array}{c}\mathrm{CC} / \mathrm{TT} \\
9.77(.002) \\
\end{array}$ \\
\hline & & & & $\begin{array}{c}\mathrm{CC} / \mathrm{CT} \\
1.60(.21) \\
\end{array}$ & $\begin{array}{c}\mathrm{CC} / \mathrm{CT} \\
0.16(.69) \\
\end{array}$ & $\begin{array}{c}\mathrm{CC} / \mathrm{TC} \\
4.62(.03) \\
\end{array}$ \\
\hline \multirow{3}{*}{$\begin{array}{l}\text { Verbal } \\
\text { learning/memory }\end{array}$} & $\begin{array}{c}\mathrm{CT} / \mathrm{TT} \\
3.64(.06)\end{array}$ & $\begin{array}{c}\text { CT/TT } \\
4.43(.04) \\
\end{array}$ & & & $\begin{array}{c}\text { CT/TT } \\
5.71(.02)\end{array}$ & \\
\hline & $\begin{array}{c}\mathrm{CC} / \mathrm{TT} \\
5.21(.02) \\
\end{array}$ & $\begin{array}{c}\text { CC/TT } \\
6.79(.009) \\
\end{array}$ & & & $\begin{array}{c}\mathrm{CC} / \mathrm{TT} \\
0.12(.73) \\
\end{array}$ & \\
\hline & $\begin{array}{c}\mathrm{CC} / \mathrm{CT} \\
0.75(.39)\end{array}$ & $\begin{array}{c}\mathrm{CC} / \mathrm{CT} \\
1.31(.25)\end{array}$ & & & $\begin{array}{c}\mathrm{CC} / \mathrm{CT} \\
1.73(.19)\end{array}$ & \\
\hline \multicolumn{7}{|l|}{ Constitutive variable } \\
\hline Premorbid intelligence & & & & & $\begin{array}{c}\mathbf{C T} / \mathbf{T T} \\
\mathbf{9 . 6 5}(\mathbf{. 0 0 2}) \\
\mathrm{CC} / \mathrm{TT} \\
1.15(.29) \\
\mathrm{CC} / \mathrm{CT} \\
1.10(.30)\end{array}$ & \\
\hline
\end{tabular}

${ }^{a}$ For CPLX2 markers with significant global association test across all genotypes (main test Table 1), we show the test statistic ( $F$ values, 1 degree of freedom) and $P$ values $(P)$ of pairwise comparisons between genotype groups. Phenotype values were standardized by Blom transformation and adjusted for sex and age, and in the case of premorbid intelligence, also for nonnative German speakers with language problems $(8.6 \%$ of the total sample; $1.9 \%$ had to be taken out completely due to severe language difficulties). Significant group comparisons $(P<.05)$ are shown in bold. 
eTable 6. Multilocus Association Analysis for $C P L X 2^{a}$

\begin{tabular}{|c|c|c|c|c|c|c|c|c|c|c|}
\hline \multirow{2}{*}{\multicolumn{2}{|c|}{$\begin{array}{l}\text { Haploblock } \\
\text { Position }\end{array}$}} & 1 & - & & & & $2 \& 3$ & & & \\
\hline & & rs2443541 & rs10072860 & $3 \mathrm{mar}$ & er haplot & pe rs1366 & $16 / \mathbf{r s 3 8 9}$ & $909 / \mathrm{rs38}$ & $2674^{(\mathrm{miR}-4}$ & 8 binding) \\
\hline \multirow{2}{*}{\multicolumn{2}{|c|}{ Modeled Effect }} & \multirow{2}{*}{$\begin{array}{l}\text { Recessive } \\
\text { T allele }\end{array}$} & \multirow{2}{*}{$\begin{array}{l}\text { Additive } \\
\text { G allele }\end{array}$} & \multicolumn{7}{|c|}{$\begin{array}{c}\text { Additive effects with respect to } \\
\text { reference haplotype TTC }(44.8 \% \text { sample frequency }) \\
\end{array}$} \\
\hline & & & & CCT & TTT & CTC & CTT & $\mathrm{CCC}$ & TCT & TCC \\
\hline \multicolumn{2}{|l|}{$\begin{array}{l}\text { Sample } \\
\text { Frequency }\end{array}$} & $\begin{array}{l}\text { MAF } \\
21.0 \%\end{array}$ & $\begin{array}{l}\text { MAF } \\
48.2 \%\end{array}$ & $31.8 \%$ & $14.8 \%$ & $2.9 \%$ & $1.8 \%$ & $1.6 \%$ & $1.3 \%$ & $1.1 \%$ \\
\hline \multicolumn{11}{|c|}{ Target Variables } \\
\hline Combined & $\begin{array}{l}\mathbf{F} \\
P\end{array}$ & $\begin{array}{c}\mathbf{8 . 2 1} \\
(.004)\end{array}$ & $\begin{array}{l}2.75 \\
(.10)\end{array}$ & $\begin{array}{l}1.44 \\
(.23)\end{array}$ & $\begin{array}{l}0.01 \\
(.91)\end{array}$ & $\begin{array}{c}17.88 \\
(<.0001)\end{array}$ & $\begin{array}{l}0.52 \\
(.47)\end{array}$ & $\begin{array}{l}0.10 \\
(.75)\end{array}$ & $\begin{array}{l}2.90 \\
(.09)\end{array}$ & $\begin{array}{l}0.57 \\
(.45)\end{array}$ \\
\hline \multicolumn{11}{|l|}{ Individual } \\
\hline \multirow{3}{*}{$\begin{array}{l}\text { Executive } \\
\text { Functioning }\end{array}$} & $\mathbf{E}$ & 0.18 & 0.09 & 0.009 & 0.002 & -0.32 & -0.12 & -0.12 & -0.21 & 0.004 \\
\hline & $\mathbf{K}$ & $\begin{array}{c}(-0.05 \\
0.42)\end{array}$ & $\begin{array}{c}(\mathbf{0 . 0 1}, \\
\mathbf{0 . 1 7})\end{array}$ & $\begin{array}{c}(-0.08, \\
0.10)\end{array}$ & $\begin{array}{c}(-0.10 \\
0.11)\end{array}$ & $\begin{array}{l}(-0.53 \\
-0.12)\end{array}$ & $\begin{array}{c}(-0.35 \\
0.11)\end{array}$ & $\begin{array}{c}(-0.40, \\
0.17)\end{array}$ & $\begin{array}{c}(-0.50, \\
0.09)\end{array}$ & $\begin{array}{c}(-0.33, \\
0.33)\end{array}$ \\
\hline & $\stackrel{t}{P}$ & $\begin{array}{l}1.52 \\
(.13) \\
\end{array}$ & $\begin{array}{l}2.31 \\
(.02) \\
\end{array}$ & $\begin{array}{l}0.20 \\
(.85) \\
\end{array}$ & $\begin{array}{l}0.05 \\
(.96) \\
\end{array}$ & $\begin{array}{l}-3.09 \\
(.002) \\
\end{array}$ & $\begin{array}{c}-1.03 \\
(.30) \\
\end{array}$ & $\begin{array}{c}-0.80 \\
(.43) \\
\end{array}$ & $\begin{array}{c}-1.38 \\
(.17) \\
\end{array}$ & $\begin{array}{l}0.02 \\
(.98)\end{array}$ \\
\hline \multirow{3}{*}{ Reasoning } & $\mathbf{E}$ & 0.24 & 0.06 & 0.09 & 0.06 & -0.31 & 0.13 & -0.02 & -0.12 & 0.09 \\
\hline & $\mathbf{K}$ & $\begin{array}{l}0.01, \\
0.46)\end{array}$ & $\begin{array}{c}(-0.01, \\
0.13)\end{array}$ & $\begin{array}{c}(0.007, \\
0.17)\end{array}$ & $\begin{array}{c}(-0.04, \\
0.15)\end{array}$ & $\begin{array}{l}(-0.50, \\
-0.12)\end{array}$ & $\begin{array}{c}(-0.10, \\
0.37)\end{array}$ & $\begin{array}{c}(-0.29, \\
0.24)\end{array}$ & $\begin{array}{c}(-0.41, \\
0.17)\end{array}$ & $\begin{array}{c}(-0.23, \\
0.41)\end{array}$ \\
\hline & $\begin{array}{l}\mathrm{t} \\
P \\
\end{array}$ & $\begin{array}{l}2.07 \\
(.04) \\
\end{array}$ & $\begin{array}{l}1.57 \\
(.12) \\
\end{array}$ & $\begin{array}{l}2.13 \\
(.03) \\
\end{array}$ & $\begin{array}{l}1.12 \\
(.26) \\
\end{array}$ & $\begin{array}{l}-3.14 \\
(.002) \\
\end{array}$ & $\begin{array}{l}1.12 \\
(.26) \\
\end{array}$ & $\begin{array}{c}-0.16 \\
(.87) \\
\end{array}$ & $\begin{array}{c}-0.82 \\
(.41) \\
\end{array}$ & $\begin{array}{l}0.54 \\
(.59) \\
\end{array}$ \\
\hline \multirow{3}{*}{$\begin{array}{l}\text { Verbal } \\
\text { Learning/ } \\
\text { Memory }\end{array}$} & $\mathbf{E}$ & 0.39 & -0.004 & 0.02 & -0.05 & -0.40 & -0.31 & 0.10 & -0.31 & -0.35 \\
\hline & $\mathbf{K}$ & $\begin{array}{l}\text { (0.15, } \\
\mathbf{0 . 6 4 )}\end{array}$ & $\begin{array}{c}(-0.08, \\
0.08)\end{array}$ & $\begin{array}{c}(-0.07, \\
0.11)\end{array}$ & $\begin{array}{c}(-0.16, \\
0.05)\end{array}$ & $\begin{array}{l}(-0.61, \\
-0.19)\end{array}$ & $\begin{array}{l}(-0.55, \\
-0.07)\end{array}$ & $\begin{array}{c}(-0.19, \\
0.39)\end{array}$ & $\begin{array}{l}(-0.61, \\
-0.01)\end{array}$ & $\begin{array}{l}(-0.68 \\
-0.02) \\
\end{array}$ \\
\hline & $\begin{array}{l}\mathrm{t} \\
P\end{array}$ & $\begin{array}{c}3.18 \\
(.002)\end{array}$ & $\begin{array}{c}-0.10 \\
(.92)\end{array}$ & $\begin{array}{l}0.39 \\
(.70)\end{array}$ & $\begin{array}{c}-0.96 \\
(.34)\end{array}$ & $\begin{array}{l}-3.71 \\
(.0002)\end{array}$ & $\begin{array}{c}-2.51 \\
(.01)\end{array}$ & $\begin{array}{l}0.68 \\
(.50) \\
\end{array}$ & $\begin{array}{c}-2.04 \\
(.04) \\
\end{array}$ & $\begin{array}{r}-2.10 \\
(.04) \\
\end{array}$ \\
\hline \multicolumn{11}{|l|}{$\begin{array}{l}\text { Constitutive } \\
\text { Variable }\end{array}$} \\
\hline \multirow{3}{*}{$\begin{array}{l}\text { Premorbid } \\
\text { Intelligence }\end{array}$} & $\mathbf{E}$ & 0.13 & 0.03 & 0.03 & -0.15 & -0.30 & -0.17 & -0.04 & -0.39 & 0.08 \\
\hline & $\mathbf{K}$ & $\begin{array}{c}(-0.11, \\
0.38)\end{array}$ & $\begin{array}{c}(-0.05, \\
0.11)\end{array}$ & $\begin{array}{c}(-0.06, \\
0.12)\end{array}$ & $\begin{array}{l}(-0.26, \\
-0.05)\end{array}$ & $\begin{array}{l}(-0.52, \\
-0.09)\end{array}$ & $\begin{array}{c}(-0.41, \\
0.08)\end{array}$ & $\begin{array}{c}(-0.34, \\
0.26)\end{array}$ & $\begin{array}{l}(-0.70, \\
-0.09)\end{array}$ & $\begin{array}{c}(-0.25 \\
0.40)\end{array}$ \\
\hline & $\begin{array}{l}\mathrm{t} \\
P\end{array}$ & $\begin{array}{l}1.07 \\
(.29)\end{array}$ & $\begin{array}{l}0.81 \\
(.42)\end{array}$ & $\begin{array}{l}0.67 \\
(.50)\end{array}$ & $\begin{array}{l}-2.81 \\
(.005)\end{array}$ & $\begin{array}{l}-2.80 \\
(.005)\end{array}$ & $\begin{array}{c}-1.35 \\
(.18)\end{array}$ & $\begin{array}{c}-0.26 \\
(.79)\end{array}$ & $\begin{array}{c}-2.50 \\
(.01)\end{array}$ & $\begin{array}{l}0.45 \\
(.65)\end{array}$ \\
\hline
\end{tabular}

Abbreviation: MAF, minor allele frequency.

${ }^{a}$ Multilocus association analysis of multivariate and univariate cognitive phenotypes on CPLX2 is based on combined analysis of all markers showing evidence for association in the single locus analyses (main Table 1). In CPLX2 haploblock 1, the 2 associated markers are highly correlated. We reduced to the marker rs2443541 and modeled a recessive effect of the minor $\mathrm{T}$ allele. All other genetic effects were modeled as additive effects on the age- and sexadjusted trait mean; premorbid intelligence was additionally adjusted for nonnative German speakers with language problems $(8.6 \%$ of the total sample; $1.9 \%$ had to be taken out completely due to severe language difficulties). Modeled additive effects were: Marker rs10072860 genotype (low linkage disequilibrium [LD] with all other associated markers) and 3 marker haplotype effect for high LD markers rs1366116/rs3892909/rs3822674 (located in CPLX2 haploblocks 2 and 3). In the sample, a total of 8 haplotypes was found. All had sample frequencies $>1 \%$, where TTC was most frequent. The reference group of our model were haplotype TTC and major rs10072860 allele homozygotes who carried maximally $1 \mathrm{~T}$ allele for rs2443541. Shown are estimated effect strength (E) with 95\% confidence interval (K) and test statistic ( $F$ or $t$ value, 1 degree of freedom) as well as $P$ value $(P)$. Like Cohen's d, E quantifies the genetically induced change of mean trait value relative to the standard deviation of the trait. All the traits have been standardized to zero mean and variance one by Blom transformation, larger trait values correspond to better performance. 
eTable 7. Association Analysis of CPLX2, Testing the Effect of One Regional Variant (CTC Haplotype in CPLX2 Haploblocks 2 \& 3) Adjusted for All Other Associated CPLX2 Regions ${ }^{\text {a }}$

\begin{tabular}{|c|c|c|c|c|}
\hline \multicolumn{2}{|l|}{ Haploblock } & 1 & - & $2 \& 3$ \\
\hline \multicolumn{2}{|l|}{ Position } & rs2443541 & rs10072860 & 3 Marker Haplotype rs1366116/rs3892909/rs3822674 ${ }_{\text {binding }}^{(\mathrm{miR}-498}$ \\
\hline \multicolumn{2}{|c|}{ Modeled Effect } & $\begin{array}{l}\text { Recessive } \\
\text { T Allele }\end{array}$ & $\begin{array}{l}\text { Additive } \\
\text { G Allele }\end{array}$ & $\begin{array}{c}\text { Additive Effects of Haplotype CTC Compared to Rest } \\
\text { (Pooling Remaining } 7 \text { Haplotypes) }\end{array}$ \\
\hline \multicolumn{2}{|l|}{$\begin{array}{l}\text { Sample } \\
\text { Frequency }\end{array}$} & $\begin{array}{l}\text { MAF } \\
21.0 \%\end{array}$ & $\begin{array}{l}\text { MAF } \\
48.2 \%\end{array}$ & $2.9 \%$ \\
\hline \multicolumn{2}{|l|}{$\begin{array}{l}\text { Significance } \\
\text { Threshold }\end{array}$} & 0.05 & 0.05 & $0.05 / 8=0.00625$ (adjustment for multiple models) \\
\hline \multicolumn{2}{|l|}{$\begin{array}{l}\text { Target } \\
\text { Variables }\end{array}$} & & & \\
\hline \multirow{2}{*}{ Combined } & $F$ & 8.21 & 6.05 & 19.29 \\
\hline & $P$ & $(.004)$ & $(.01)$ & $(<.0001)$ \\
\hline \multicolumn{5}{|l|}{ Individual } \\
\hline \multirow{4}{*}{$\begin{array}{l}\text { Executive } \\
\text { Functioning }\end{array}$} & $\mathbf{E}$ & 0.17 & 0.09 & $-\mathbf{0 . 3 2}$ \\
\hline & $\mathbf{K}$ & $(-0.06,0.40)$ & $(0.02,0.16)$ & $(-0.52,-0.12)$ \\
\hline & $\mathrm{t}$ & 1.43 & 2.58 & -3.10 \\
\hline & $P$ & $(.15)$ & $(.01)$ & $(.002)$ \\
\hline & & & & \\
\hline \multirow{4}{*}{ Reasoning } & $\mathbf{E}$ & 0.26 & 0.09 & $-\mathbf{0 . 3 4}$ \\
\hline & $\mathbf{K}$ & $(0.04,0.48)$ & $(0.02,0.15)$ & $(-0.53,-0.15)$ \\
\hline & $\mathbf{t}$ & 2.31 & 2.69 & -3.56 \\
\hline & $P$ & $(.02)$ & $(.007)$ & $(.0004)$ \\
\hline \multirow{4}{*}{$\begin{array}{l}\text { Verbal } \\
\text { Learning/ } \\
\text { Memory }\end{array}$} & $\mathbf{E}$ & 0.37 & 0.01 & -0.39 \\
\hline & $\mathbf{K}$ & $(0.13,0.61)$ & $(-0.06,0.08)$ & $(-0.59,-0.18)$ \\
\hline & $\mathrm{t}$ & 2.99 & 0.31 & -3.66 \\
\hline & $P$ & $(.003)$ & $(.76)$ & $(.0003)$ \\
\hline \multicolumn{2}{|l|}{$\begin{array}{l}\text { Constitutive } \\
\text { Variable }\end{array}$} & & & \\
\hline \multirow{4}{*}{$\begin{array}{l}\text { Premorbid } \\
\text { Intelligence }\end{array}$} & $\mathbf{E}$ & 0.11 & 0.06 & -0.28 \\
\hline & $\mathbf{K}$ & $(-0.14,0.35)$ & $(-0.010,0.13)$ & $(-0.49,-0.07)$ \\
\hline & $\mathrm{t}$ & 0.86 & 1.76 & -2.62 \\
\hline & $P$ & $(.39)$ & $(.08)$ & $(.009)$ \\
\hline
\end{tabular}

Abbreviation: MAF, minor allele frequency.

${ }^{a}$ Presented is the multilocus association analysis of multivariate and univariate cognitive phenotypes on CPLX2. Considered was the class of multilocus models which selects 1 of the 8 observed haplotypes at rs1366116/rs3892909/rs3822674 to compare it with respect to all remaining haplotypes, pooling the latter into 1 group. We tested the additive effect of haplotype CTC (with multiple testing adjustment) on the ageand sex-adjusted trait mean (premorbid intelligence is additionally adjusted for nonnative German speakers with language problems, amounting to $8.6 \%$ of the total sample; $1.9 \%$ had to be taken out completely due to severe language difficulties), while adjusting also for the effects of the other 2 associated CPLX2 regions. The latter are represented by rs2443541 genotype (recessive effect of minor allele) and rs10072860 genotype (additive effect of minor allele). The reference group has at most $1 \mathrm{~T}$ allele for rs2443541, is homozygous for the major allele of rs10072860 and does not have haplotype CTC at rs1366116/rs3892909/rs3822674. Shown are estimated effect strength (E) with $95 \%$ confidence interval $(K)$ and test statistic (F or t value, 1 degree of freedom) as well as $P$ value $(P)$. Like Cohen's d, E quantifies the genetically induced change of mean trait 
value relative to the standard deviation of the trait. Prior to statistical analysis, all traits have been standardized to zero mean and variance one by Blom transformation. Larger trait values correspond to better performance.

${ }^{\mathrm{b}} \mathrm{A}$ total of 8 models are possible, each comparing a different haplotype to the respective pooled group of all other haplotypes. 


\section{eTable 8. Species Conservation ${ }^{\mathrm{a}}$}

\begin{tabular}{|c|c|c|c|c|c|c|c|c|c|c|c|}
\hline & $\begin{array}{l}\infty \\
0 \\
0 \\
0 \\
0 \\
00 \\
0\end{array}$ & 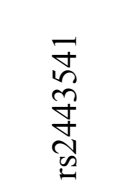 & 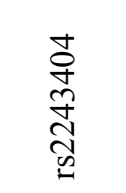 & 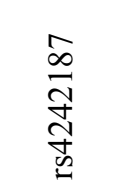 & 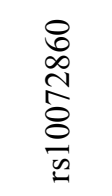 & 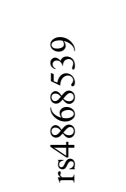 & $\begin{array}{l}0 \\
\overline{0} \\
0 \\
\frac{0}{2} \\
0\end{array}$ & 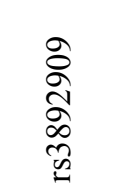 & 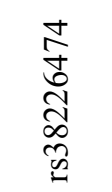 & 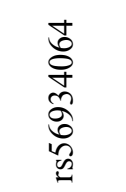 & 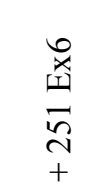 \\
\hline Human & $\mathrm{A}$ & $\mathrm{C}$ & $\mathrm{C}$ & $\mathrm{C}$ & $\mathrm{T}$ & G & $\mathrm{T}$ & $\mathrm{T}$ & $\mathrm{T}$ & $\mathrm{A}$ & $\mathrm{G}$ \\
\hline Chimp & $\mathrm{A}$ & $\mathrm{T}$ & $\mathrm{T}$ & $\mathrm{T}$ & $\mathrm{G}$ & $\mathrm{G}$ & $\mathrm{C}$ & $\mathrm{T}$ & $\mathrm{N}$ & $\mathrm{N}$ & $\mathrm{N}$ \\
\hline Orangutan & $\mathrm{A}$ & $\mathrm{T}$ & $\mathrm{T}$ & $\mathrm{T}$ & $\mathrm{N}$ & $\mathrm{G}$ & $\mathrm{T}$ & $\mathrm{T}$ & $\mathrm{T}$ & $\mathrm{G}$ & $\mathrm{N}$ \\
\hline Macaque & $\mathrm{A}$ & $\mathrm{C}$ & $\mathrm{T}$ & $\mathrm{T}$ & $\mathrm{G}$ & $\mathrm{G}$ & $\mathrm{T}$ & $\mathrm{T}$ & $\mathrm{T}$ & $\mathrm{G}$ & $\mathrm{N}$ \\
\hline Rhesus & $\mathrm{A}$ & $\mathrm{C}$ & $\mathrm{T}$ & $\mathrm{T}$ & $\mathrm{G}$ & $\mathrm{G}$ & $\mathrm{T}$ & $\mathrm{T}$ & $\mathrm{T}$ & $\mathrm{T}$ & $\mathrm{G}$ \\
\hline Bushbaby & $\mathrm{A}$ & $\mathrm{C}$ & $\mathrm{C}$ & $\mathrm{T}$ & $\mathrm{T}$ & $=$ & $\mathrm{T}$ & $=$ & $=$ & $=$ & $=$ \\
\hline Rat & $=$ & $\mathrm{C}$ & $\mathrm{C}$ & - & $\mathrm{C}$ & - & $\mathrm{T}$ & $\mathrm{C}$ & $\mathrm{T}$ & $\mathrm{T}$ & $\mathrm{G}$ \\
\hline Mouse & $=$ & $\mathrm{C}$ & $\mathrm{C}$ & $=$ & $\mathrm{C}$ & - & $\mathrm{T}$ & $\mathrm{T}$ & $\mathrm{T}$ & $\mathrm{T}$ & $\mathrm{G}$ \\
\hline Dog & $=$ & $\mathrm{C}$ & $\mathrm{T}$ & $\mathrm{T}$ & $\mathrm{A}$ & - & $\mathrm{C}$ & $\mathrm{T}$ & $\mathrm{T}$ & $\mathrm{T}$ & $\mathrm{G}$ \\
\hline Cat & $=$ & $=$ & $\mathrm{T}$ & $\mathrm{T}$ & A & $\mathrm{G}$ & $\mathrm{C}$ & $\mathrm{T}$ & $\mathrm{T}$ & $\mathrm{T}$ & $\mathrm{G}$ \\
\hline Cow & $=$ & $\mathrm{C}$ & $\mathrm{T}$ & $\mathrm{G}$ & $=$ & $\mathrm{A}$ & $\mathrm{T}$ & $\mathrm{T}$ & $\mathrm{T}$ & $\mathrm{T}$ & $\mathrm{G}$ \\
\hline Tenrec & $=$ & $\mathrm{C}$ & $\mathrm{T}$ & $\mathrm{A}$ & $=$ & $\mathrm{N}$ & $\mathrm{C}$ & $\mathrm{C}$ & $\mathrm{T}$ & $\mathrm{T}$ & - \\
\hline $\begin{array}{l}\text { MAF } \\
(\mathrm{CON})\end{array}$ & $\mathrm{T}: 0.41$ & $\mathrm{~T}: 0.22$ & $\mathrm{~T}: 0.21$ & $\mathrm{~T}: 0.34$ & G:0.48 & A: 0.47 & C:0.39 & $\mathrm{C}: 0.36$ & $\mathrm{~T}: 0.50$ & G:0.27 & C: 0.28 \\
\hline $\begin{array}{l}\text { MAF } \\
\text { (SCZ) }\end{array}$ & $\mathrm{T}: 0.42$ & $\mathrm{~T}: 0.21$ & $\mathrm{~T}: 0.20$ & $\mathrm{~T}: 0.33$ & G:0.48 & A: 0.47 & C:0.38 & C: 0.36 & $\mathrm{~T}: 0.50$ & G:0.27 & C: 0.27 \\
\hline $\begin{array}{l}\text { MAF } \\
(\mathrm{CEU})^{\mathrm{b}}\end{array}$ & $\mathrm{T}: 0.37$ & $\mathrm{~T}: 0.31$ & $\mathrm{~T}: 0.32$ & $\mathrm{~T}: 0.47$ & $\mathrm{~T}: 0.47$ & A:0.44 & C:0.44 & C:0.39 & C:0.47 & ND & ND \\
\hline
\end{tabular}

Abbreviations: CON, control subjects; MAF, minor allele frequency; N, no sequence available; ND, no data available; SCZ, schizophrenic subjects; =, 1 or more nonalignable basepairs in this region; -, no bases in the aligned species, possibly due to the lineage-specific deletion between the aligned block in the aligning species.

${ }^{a}$ The table shows single base pair alignments at the exact position of the analyzed SNPs in CPLX2 for selected species. MAFs in CON and SCZ subjects are comparable and similar to the ones reported in the HapMap ${ }^{\mathrm{b}}$ project.

${ }^{\mathrm{b}}$ Based on HapMap data. 


\section{eFigure 1A}
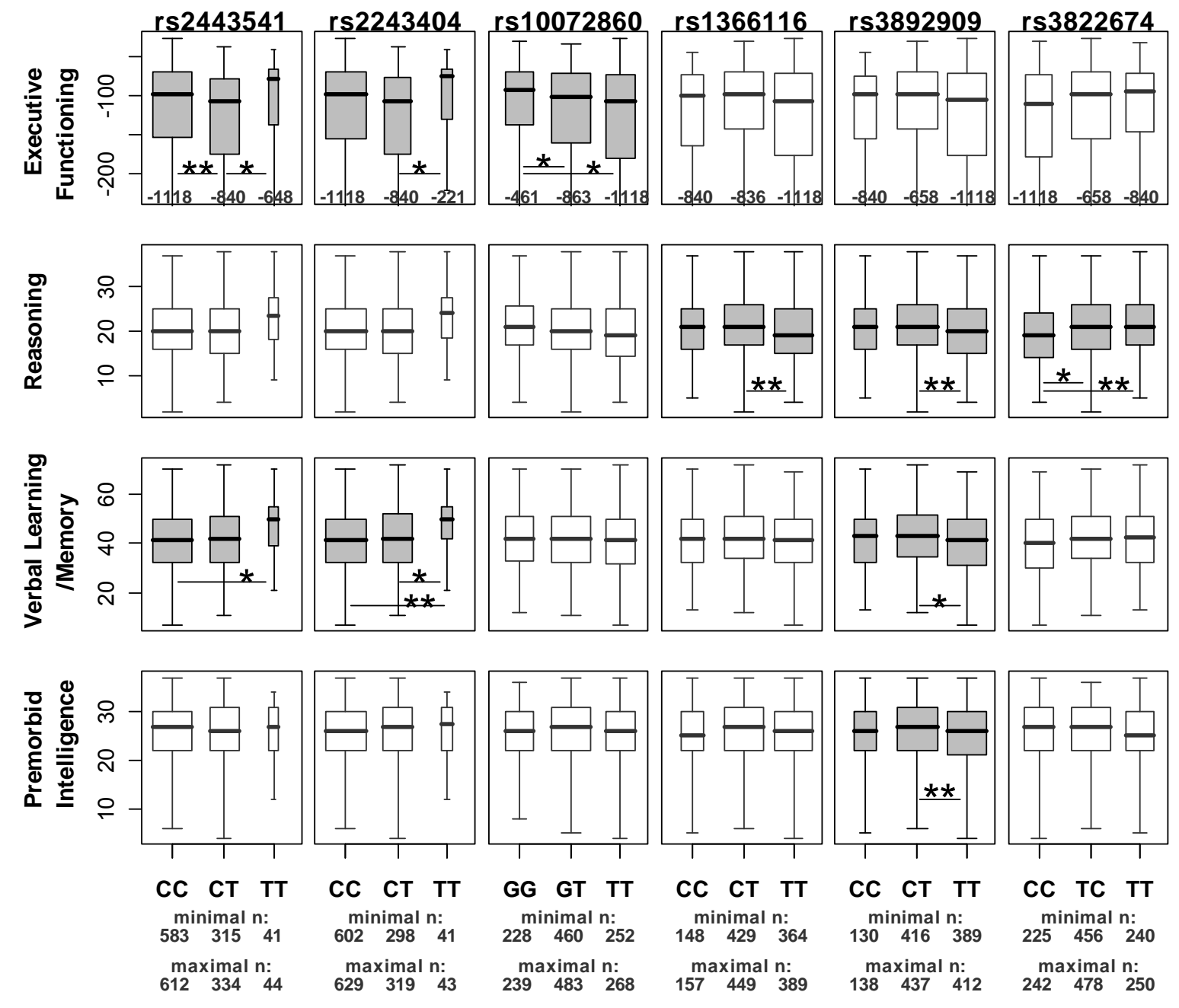

\section{eFigure 1B}

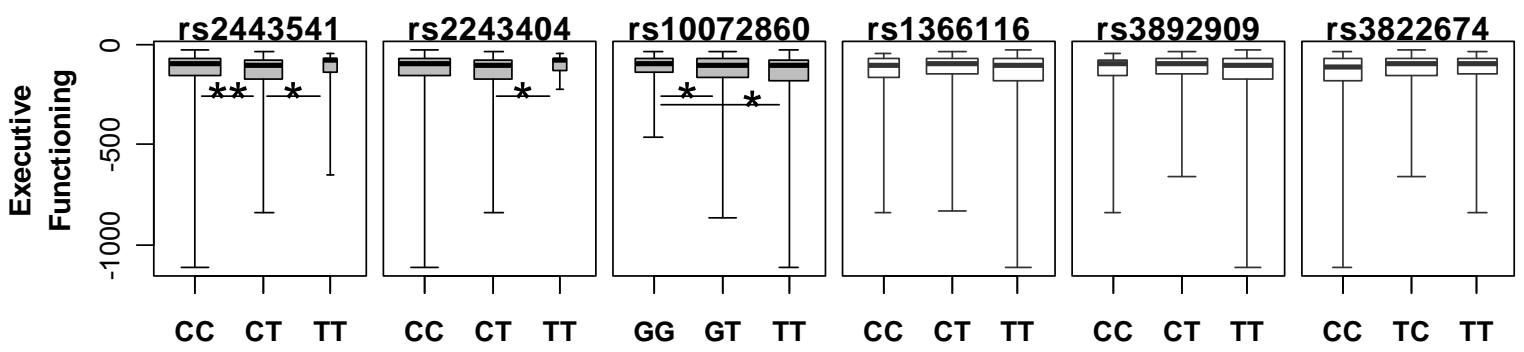

eFigure 1. Influence of CPLX2 Genotypes on Cognitive Measures of Schizophrenic Patients

A. Presented are box plots of target and constitutive phenotypes (raw data), stratified by CPLX2 genotypes. Higher phenotype values correspond to better cognition. For executive functioning, we display negative execution times, omit (for illustration purposes) the lower $12 \%$ of the data, and give instead the lowest extreme value. Highlighted are phenotype-genotype pairs with significant global association test (Table, main text), indicating significant pairwise genotype comparison by $* P<.05$ and $* * P<.01$ (eTable 5). Statistical testing was performed on the Blom-transformed phenotypes, adjusting for age and sex, and in the case of premorbid intelligence, also for nonnative German speakers with language problems $(8.6 \%$ of the total sample; $1.9 \%$ had to be taken out completely due to severe language difficulties). Minimal and maximal available sample sizes (n) are displayed at the bottom of the graph. B. Additional full (uncropped) presentation of executive functioning data. 


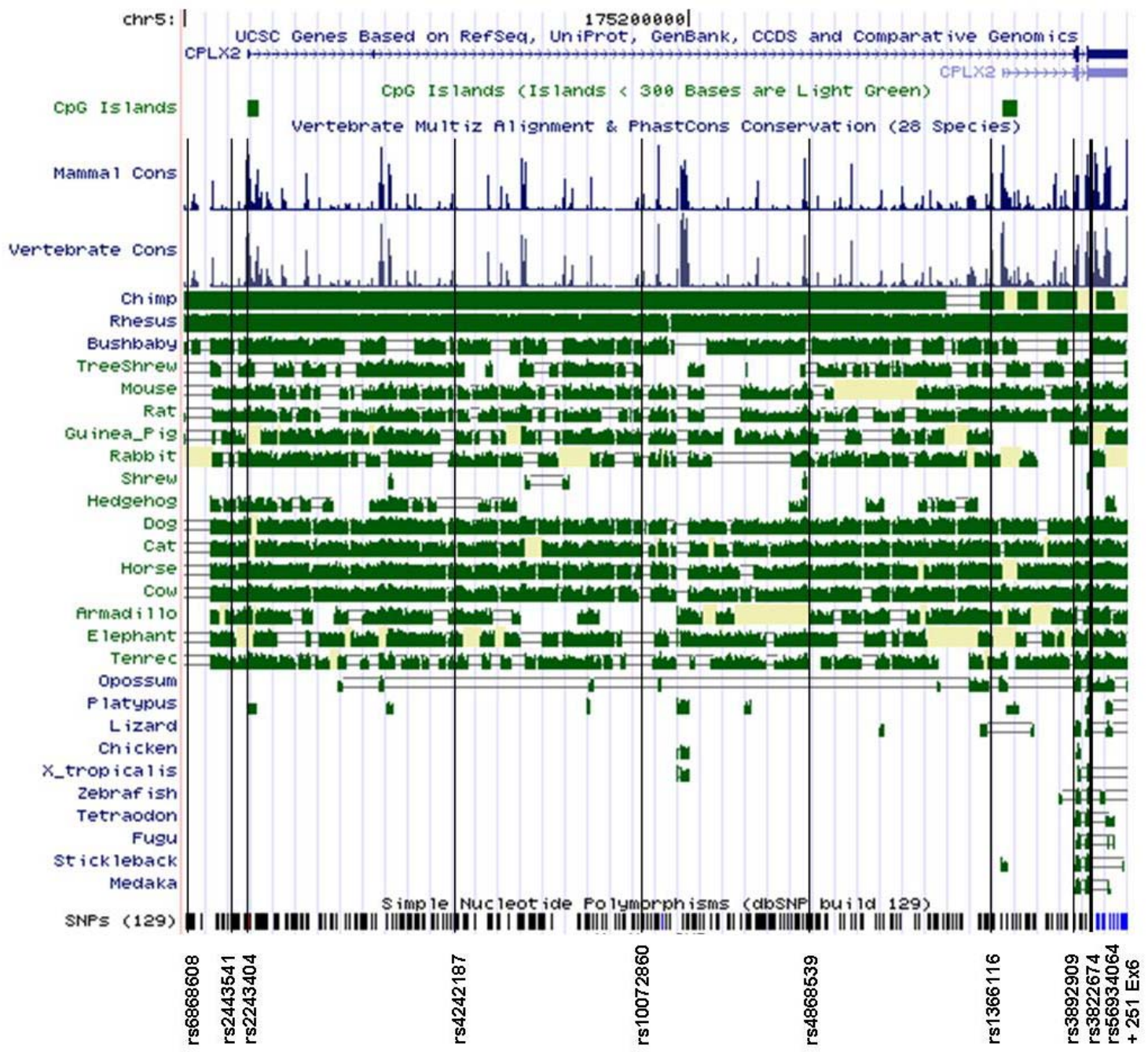

eFigure 2. Conservation Status of the CPLX2 Gene Across 28 Species

The figure shows the mammalian and vertebrate conservation of $C P L X 2$. Also displayed are the different transcripts, predicted $\mathrm{CpG}$ islands, and the analyzed markers. The exons of CPLX2 seem to be highly conserved (high blue peaks). For single-marker analysis, see eTable 8. 
eReferences. Bibliography for the Supplementary Methods Section

1. Ashcroft R. The Declaration of Helsinki. In: Emanuel E, Grady C, Crouc, R, eds. The Oxford Textbook of Clinical Research Ethics. New York, NY: Oxford University Press; 2007.

2. American Psychiatric Association. Diagnostic and Statistical Manual of Mental Disorders. 4th ed. Washington, DC: American Psychiatric Association; 1994.

3. Wittchen H-U, Zaudig M, Fydrich T. SCID-I: Structured Clinical Interview for DSM-IV Disorders. Goettingen, Germany: Hogrefe; 1997.

4. McGlashan TH, Fenton WS. Classical subtypes for schizophrenia: literature review for DSM-IV. Schizophr Bull. 1991;17(4):609-632.

5. Kay SR, Fiszbein A, Opler LA. The Positive and Negative Syndrome Scale (PANSS) for schizophrenia. Schizophr Bull. 1987;13(2):261-276.

6. Franke GH. Brief Symptom Inventory (BSI). Goettingen, Germany: Beltz; 2000.

7. Laux L, Glanzmann P, Schaffner P, Spielberger CD. State-Trait Anxiety Inventory (STAI). Weinheim, Germany: Beltz; 1981.

8. Kupfer J, Brosig B, Braehler E. Toronto Alexithymia Scale 26 (TAS-26). Goettingen, Germany: Hogrefe; 2001.

9. Guy W. Abnormal involuntary movement scale (AIMS). ECDEU Assessment Manual for Psychopharmacology, Revised. Rockville, MD: National Institute of Mental Health; 1976.

10. War Department. Army Individual Test Battery Manual of Directions and Scoring. Washington, DC: War Department, Adjutant General's Office; 1944.

11. Reitan RM. The validity of the Trail Making Test as an indicator of organic brain damage. Percept Motor Skills. 1958;8:271-276.

12. Horn W. General Performance Test (translated from German [LPS]). 2nd ed. Goettingen, Germany: Hogrefe; 1983.

13. Helmstaedter $C$, Lendt $M$, Lux S. Verbal Learning and Memory Test (VLMT). Goettingen, Germany: Beltz; 2001.

14. Lehrl S. Multiple Choice Vocabulary Intelligence Test (translated from German [MWT-B]). Balingen, Germany: Spitta Verlag; 1999.

15. Wechsler D. Wechsler Adult Intelligence Scale [WAIS]. 3rd ed. San Antonio, TX: Psychological Corporation; 1997.

16. Tewes U. German Adaption of the Revised Wechsler Adult Intelligence Scale (HAWIE-R). Bern, Switzerland: Huber; 1991.

17. Aschenbrenner S, Tucha, O, Lange, K. Regensburger Verbal Fluency Test (translated from German). Goettingen, Germany: Hogrefe; 2000.

18. Calabrese P, Kessler, J. DemTect Screening Instrument for Supporting of the Diagnostics of Dementia DemTec. Karlsruhe, Germany: Pfizer GmbH; 2000.

19. Chapman RL. The MacQuarrie test for mechanical ability. Psychometrika. 1948;13(3):175-179.

20. Zimmermann P, Fimm B. Test for Attentional Performance (translated from German [TAP]). Freiburg, Germany: PSYTEST; 1993. 
21. Chen EY, Shapleske J, Luque R, McKenna PJ, Hodges JR, Calloway SP, Hymas NF, Dening TR, Berrios GE. The Cambridge Neurological Inventory: a clinical instrument for assessment of soft neurological signs in psychiatric patients. Psychiatry Res. 1995;56(2):183-204.

22. Bartels C, Mertens N, Hofer S, Merboldt KD, Dietrich J, Frahm J, Ehrenreich H. Callosal dysfunction in amyotrophic lateral sclerosis correlates with diffusion tensor imaging of the central motor system. Neuromuscul Disord. 2008;18(5):398-407.

23. Barnes TR. A rating scale for drug-induced akathisia. Br J Psychiatry. 1989;154:672-676.

24. Simpson GM, Angus JW. A rating scale for extrapyramidal side effects. Acta Psychiatr Scand Suppl. 1970;212:11-19.

25. Simpson GM, Lee JH, Zoubok B, Gardos G. A rating scale for tardive dyskinesia. Psychopharmacology (Berl). 1979;64(2):171-179.

26. Barrett JC, Fry B, Maller J, Daly MJ. Haploview: analysis and visualization of LD and haplotype maps. Bioinformatics. 2005;21(2):263-265.

27. Armitage P, Berry G, Matthews JNS. Statistical Methods in Medical Research. 4th ed. Oxford, England: Blackwell; 2002.

28. Cronbach LJ. Coefficient alpha and the internal structure of tests. Psychometrika. 1951;16:297334.

29. Blom G. Statistical Estimates and Transformed Beta Variables. New York, NY: John Wiley \& Sons Inc; 1958.

30. Dudbridge F. Likelihood-based association analysis for nuclear families and unrelated subjects with missing genotype data. Hum Hered. 2008;66(2):87-98.

31. Purcell S, Neale B, Todd-Brown K, Thomas L, Ferreira MA, Bender D, Maller J, Sklar P, de Bakker PI, Daly MJ, Sham PC. PLINK: a tool set for whole-genome association and populationbased linkage analyses. Am J Hum Genet. 2007;81(3):559-575.

32. SAS Institute, Inc. SAS Software, Version 9.1 of the SAS System for Windows. Cary, NC: SAS Institute Inc; 2001.

33. Altschul SF, Gish W, Miller W, Myers EW, Lipman DJ. Basic local alignment search tool. J Mol Biol. 1990;215(3):403-410.

34. Wang X, Zhang J, Li F, Gu J, He T, Zhang X, Li Y. MicroRNA identification based on sequence and structure alignment. Bioinformatics. 2005;21(18):3610-3614.

35. Lewis BP, Burge CB, Bartel DP. Conserved seed pairing, often flanked by adenosines, indicates that thousands of human genes are microRNA targets. Cell. 2005;120(1):15-20.

36. Siepel A, Bejerano G, Pedersen JS, Hinrichs AS, Hou M, Rosenbloom K, Clawson H, Spieth J, Hillier LW, Richards S, Weinstock GM, Wilson RK, Gibbs RA, Kent WJ, Miller W, Haussler D. Evolutionarily conserved elements in vertebrate, insect, worm, and yeast genomes. Genome Res. 2005;15(8):1034-1050.

37. McIlwain KL, Merriweather MY, Yuva-Paylor LA, Paylor R. The use of behavioral test batteries: effects of training history. Physiol Behav. 2001;73(5):705-717.

38. Morris R. Developments of a water-maze procedure for studying spatial learning in the rat. $J$ Neurosci Methods. 1984;11(1):47-60. 


\section{A CAG REPEAT POLYMORPHISM OF KCNN3 PREDICTS SK3 CHANNEL FUNCTION AND COGNITIVE PERFORMANCE IN SCHIZOPHRENIA}

\subsection{OVerview of Project II}

Small-conductance, $\mathrm{Ca}^{2+}$-activated $\mathrm{K}^{+}$channels (SK) are voltage independent $\mathrm{K}^{+}$channels that can be found in different types of neurons and are activated by raises in intracellular calcium. For appropriate action, they need to be assembled in a multi-protein complex comprising casein kinase 2, protein phosphatase 2A, and calmodulin that is constitutively bound to the carboxy-terminus (C-terminus) (Pedarzani and Stocker, 2008). They contribute to the afterhyperpolarization that follows action potentials and control repetitive firing patterns. It is strongly believed that they are important players in controlling dendrite excitability, synaptic transmission and synaptic plasticity (Faber, 2009).

Three SK channel subtypes are expressed in mammalian brain which share high homology in the transmembrane domain but show divergence in the $\mathrm{C}$ - and $\mathrm{N}$-terminus. SK1 expression is restricted to the brain, whereas SK2 and SK3 are as well expressed in peripheral tissue (Kohler et al, 1996). In the brain, SK1, SK2 and SK3 have partially overlapping but distinct distribution patterns, with SK1 and SK2 frequently expressed in the same neurons, and SK3 presenting a complementary distribution (Rimini et al, 2000; Sailer et al, 2004). The exact localization is controversially discussed; some immunofluorescence studies suggest a presynaptic localization (Obermair et al, 2003; Roncarati et al, 2001) whereas more functional studies also support a post-synaptic localization (Cai et al, 2004; Faber et al, 2005).

Several studies in vitro and in vivo have shown that blocking SK channels with apamin leads to a conversion from tonic to burst firing in cell culture (Wolfart et al, 2001), and to enhanced LTP and improved learning in mice (Stackman et al, 2002). Unfortunately, the specific contribution of the subtypes is difficult to assess due to the lack of subtype-specific pharmacological agents. To circumvent this problem of specificity, some mouse models have been analyzed so far and have shed some light on specific SK channel function. Mice overexpressing SK2 show selective deficits in Morris water maze and fear conditioning (Hammond et al, 2006). Conditional overexpression of SK3 leads to defects in respiration and parturition (Bond et al, 2000), whereas doxycycline-dependent SK3 null mutant mice show alterations in tests of depressive behavior and cognition (Jacobsen et al, 2009; Jacobsen et al, 
2008). Interestingly, aged mice, showing deficits in learning and memory tasks, have increased levels of SK3 in the brain, suggesting a contribution of SK3 to the age-dependent decline in cognitive functions (Blank et al, 2003).

Considering these functional consequences of SK dysregulation, it seems reasonable to implicate these ion channels as well in disease. Indeed, there has been a major focus on SK3. The encoding gene KCNN3 maps to chromosome 1q21-22 (Austin et al, 1999) and encodes a protein of 731 amino acids containing two adjacent polyglutamine arrays in its $\mathrm{N}$-terminal domain whereby the second one is highly polymorphic (Chandy et al, 1998; Wittekindt et al, 1998). Taken into account that many neurological disorders are caused by triplet expansions, SK3 is an interesting candidate (Orr and Zoghbi, 2007). Several association studies have been performed, linking the SK3 polyglutamine repeat, among others, to migraine (Curtain et al, 2005), anorexia nervosa (Koronyo-Hamaoui et al, 2004), ataxia (Figueroa et al, 2001), epilepsy (Vijai et al, 2005) and bipolar disorder (Guy et al, 1999).

However, most studies focused for several reasons on schizophrenia. First, there is evidence for a major susceptibility locus at chromosomal location 1q21-22 (e.g. Brzustowicz et al, 2000; Rosa et al, 2002). Second, a role of trinucleotide repeats in psychotic disorders has been discussed for quite some time (e.g. Vincent et al, 2000). Third, the findings on SK channel function provide interesting links to schizophrenia. Hyperpolarization of the membrane potential keeps NMDA receptors blocked. Hyperactive SK channels can therefore be expected to induce NMDA hypofunction. In contrast, blockage of SK channels in dopaminergic neurons induces bursting actions potentials, an activity associated with excess dopamine release (Shepard and Bunney, 1991). Thus, dysregulation of SK channels might lead to features that are implicated in the pathogenesis of schizophrenia (Gargus, 2006).

The initial study on this topic by Chandy in 1998 indeed found a significant association of long alleles with schizophrenia (Chandy et al, 1998). However, after more than 20 studies have been conducted in different populations with contradictory results, a meta-analysis by Glatt and colleagues in 2003 concluded that the CAG repeat length in KCNN3 does not influence the risk for schizophrenia (Glatt et al, 2003). On the phenotype level there are only a few studies; longer CAG repeat length has been associated with increased negative symptoms (Cardno et al, 1999), anergia and paranoid symptoms (Ritsner et al, 2002) in samples much smaller than ours. 
Based on these findings, we decided to analyze the CAG repeat in exon 1 of KCCN3 in our GRAS population. We hypothesized that the repeat does not contribute to schizophrenia risk, but influences selective phenotypes in a normal range as proposed for sequence repeats (Fondon et al, 2008). Because of the modulatory function of SK3 expression on cognition in mice (Blank et al, 2003), we focused on cognitive readouts in our population. Furthermore, we aimed to understand the biological role of this repeat in channel expression and electrophysiological properties.

In this study we were able to show for the first time a functional consequence of the CAG repeat length in KCCN3 on channel conductance. Long repeats lead to a less functional channel and are associated with better cognitive performance in humans. In contrast, SK3 overexpressing mice, modeling an increased channel activity, show remarkable deficits in similar cognitive tasks. 


\subsection{Original Publication}

Grube S*, Gerchen MF*, Adamcio B*, Pardo LA, Martin S, Malzahn D, Papiol S, Begemann M, Ribbe K, Friedrichs H, Radyushkin KA, Müller M, Benseler F, Riggert J, Falkai P, Bickeböller H, Brose N, Nave KA, Stühmer W, Ehrenreich H (2011). A CAG repeat polymorphism of KCNN3 predicts SK3 channel function and cognitive performance in schizophrenia. EMBO Molecular Medicine, in press.

* Indicates equal contribution for the publication

Personal contribution:

I was involved in the design of the study, interpretation of results, and the experiments. I conducted the whole genetic part of the project and constructed the vectors for the whole-cell patch clamp measurements. I was the responsible person for designing and finalizing all display items, preparation of the manuscript, including review of the literature, and the whole submission/revision process until acceptance. 


\title{
A CAC repeat polymorphism of $K C N_{3}$ predicts SK3 channel function and cognitive performance in schizophrenia
}

\author{
Sabrina Grube ${ }^{1 \dagger}$, Martin F. Gerchen ${ }^{1 \dagger}$, Bartosz Adamcio ${ }^{1 \dagger}$, Luis A. Pardo ${ }^{2}$, Sabine Martin ${ }^{2,3}$, \\ Dörthe Malzahn ${ }^{4}$, Sergi Papiol ${ }^{1,3,5}$, Martin Begemann ${ }^{1}$, Katja Ribbe ${ }^{1}$, Heidi Friedrichs ${ }^{1}$, \\ Konstantin A Radyushkin ${ }^{1,3}$, Michael Müller ${ }^{3,6}$, Fritz Benseler ${ }^{7}$, Joachim Riggert ${ }^{8}$, \\ Peter Falkai ${ }^{3,9}$, Heike Bickeböller ${ }^{4}$, Klaus-Armin Nave ${ }^{3,5}$, Nils Brose ${ }^{3,7}$, \\ Walter Stühmer ${ }^{2,3 * *}$, Hannelore Ehrenreich ${ }^{1,3 *}$
}

Keywords: higher cognitive testing; mouse behaviour; neuropsychology; small conductance calcium-activated potassium channel; whole-cell patch clamp

DOI 10.1002/emmm.201100135

Received October 11, 2010

Revised February 24, 2011

Accepted February 25, 2011
$K C N N 3$, encoding the small conductance calcium-activated potassium channel SK3, harbours a polymorphic CAG repeat in the amino-terminal coding region with yet unproven function. Hypothesizing that KCNN3 genotypes do not influence susceptibility to schizophrenia but modify its phenotype, we explored their contribution to specific schizophrenic symptoms. Using the Göttingen Research Association for Schizophrenia (GRAS) data collection of schizophrenic patients $(n=1074)$, we performed a phenotype-based genetic association study (PGAS) of KCNN3. We show that long CAG repeats in the schizophrenic sample are specifically associated with better performance in higher cognitive tasks, comprising the capacity to discriminate, select and execute $(p<0.0001)$. Long repeats reduce SK3 channel function, as we demonstrate by patch-clamping of transfected HEK293 cells. In contrast, modelling the opposite in mice, i.e. Kcnn3 overexpression/channel hyperfunction, leads to selective deficits in higher brain functions comparable to those influenced by SK3 conductance in humans. To conclude, KCNN3 genotypes modify cognitive performance, shown here in a large sample of schizophrenic patients. Reduction of SK3 function may constitute a pharmacological target to improve cognition in schizophrenia and other conditions with cognitive impairment.
(1) Divison of Clinical Neuroscience, Max Planck Institute of Experimental Medicine, Göttingen, Germany.

(2) Department of Molecular Biology of Neuronal Signals, Max Planck Institute of Experimental Medicine, Göttingen, Germany.

(3) DFG Research Center for Molecular Physiology of the Brain (CMPB), Göttingen, Germany.

(4) Department of Genetic Epidemiology, Medical School, Georg-AugustUniversity, Göttingen, Germany.

(5) Department of Neurogenetics, Max Planck Institute of Experimental Medicine, Göttingen, Germany.

(6) Department of Neuro- and Sensory Physiology, Georg-August-University, Göttingen, Germany.
(7) Department of Molecular Neurobiology, Max Planck Institute of Experimental Medicine, Göttingen, Germany.

(8) Department of Transfusion Medicine, Georg-August-University, Göttingen, Germany.

(9) Department of Psychiatry and Psychotherapy, Georg-August-University, Göttingen, Germany.

*Corresponding author: Tel: +49551 3899 628; Fax: +49 5513899 670;

E-mail: ehrenreich@em.mpg.de

**Corresponding author: $\underline{\text { Q1. }}$

E-mail: ws@em.mpg.de

${ }^{\dagger}$ Authors contributed equally to this work 


\section{INTRODUCTION}

SK3, also known as $\mathrm{K}_{\mathrm{Ca}} 2.3$, belongs to the family of tetrameric, small conductance calcium-activated potassium channels (SK) and is encoded by the KCNN3 gene on human chromosome 1q21.3. This gene is characterized by a polymorphic CAG repeat in the $N$-terminal coding region (Chandy et al, 1998) whose functional significance has not been elucidated yet (Bond et al, 2000; Frei et al, 2006).

Small conductance calcium-activated potassium channels are widely disseminated in the central nervous system and in peripheral tissues (Kohler et al, 1996). In the brain, SK1, SK2 and SK3 are expressed in a partially overlapping but distinct pattern, with comparable regional distributions in rats (Kohler et al, 1996; Stocker \& Pedarzani, 2000), mice (Sailer et al, 2004), and humans (Dror et al, 1999; Rimini et al, 2000). SK3 mRNA expression is sparse in the neocortex, but prominent in lateral septal and septohippocampal nuclei, amygdala, thalamus, caudate-putamen, substantia nigra pars compacta, in monoaminergic neurons in the brain stem, including ventral tegmental area, dorsal raphe nucleus, locus coeruleus, hypothalamus and in Golgi interneurons in the cerebellum (Stocker \& Pedarzani, 2000). Expression of SK3 in hippocampus is particularly found in the dentate hilus and the stratum lucidum of CA3 (Sailer et al, 2004). In the ventral midbrain, SK3 mRNA is confined to areas that contain dopaminergic neurons (Sarpal et al, 2004).

Neuronal SK channels are involved in the regulation of excitability and firing patterns, neurotransmitter release, and synaptic plasticity (for reviews see (Faber, 2009; Pedarzani \& Stocker, 2008; Stocker et al, 2004)). In dopaminergic neurons of the substantia nigra, SK3 controls frequency and precision of intrinsic pacemaker activity as shown in mouse brain slices (Wolfart et al, 2001). Apamin, a selective SK channel blocker, enhances bursting activity of these neurons in rats in vivo (Ji \& Shepard, 2006). Due to the lack of SK subtype-specific pharmacological agents, the differential contribution of SK1, SK2 and SK3 to behaviour and cognition is difficult to assess, but mouse models have shed some light on SK3 function. For example, SK3 is upregulated in hippocampus of aged mice, and its downregulation by antisense oligonucleotides reverses agerelated deficits in hippocampus-dependent memory tasks and long-term potentiation (Blank et al, 2003). Abnormal respiration after hypoxia and disturbed parturition were reported in SK3 overexpressing mice but no striking deficits were identified in SK3 null mutants (Bond et al, 2000). However, doxycyclinedependent SK3 null mutant mice exhibited increased extracellular striatal dopamine, enhanced hippocampal serotonin release, and reduced hippocampal brain-derived neurotrophic factor (BDNF) expression. These mice, exposed to chronic highdose doxycycline feeding, showed also alterations in tests of depressive behaviour and cognition (Jacobsen et al, 2008, 2009).

Even though the functional significance of a variable glutamine repeat length in the SK3 channel protein has remained unclear, it has been investigated in the context of certain pathologies in humans. An association of the CAG repeat length of KCNN3 has been discussed for anorexia nervosa (Koronyo-Hamaoui et al, 2002, 2007), migraine (Curtain et al, 2005; Mossner et al, 2005), ataxia (Figueroa et al, 2001), epilepsy (Sander et al, 1999; Vijai et al, 2005) and schizophrenia (Chandy et al, 1998) but most results remain quite equivocal. Regarding schizophrenia, a disease-associated excess of longer CAG repeats was reported (Chandy et al, 1998). In contrast, family-based studies claimed a connection between shorter CAG repeats and schizophrenia (Stober et al, 1998). A meta-analysis concluded that overall, the CAG repeat length of KCNN3 does not augment the risk of schizophrenia, although a small but significant risk appeared associated with CAG repeats longer than the modal value (Glatt et al, 2003). On the other hand, a longer CAG repeat length has been linked to increased negative symptoms in a British schizophrenic sample (Cardno et al, 1999), and in Jewish schizophrenic patients, where also anergia and paranoid symptoms were found associated (Ritsner et al, 2002).

Based on the above delineated effects of SK3 on cognition in mice (Blank et al, 2003), we hypothesized that SK3 genotypes also influence cognitive performance in humans. In particular, we assumed that SK3 genotypes might modify higher cognition in schizophrenia rather than contributing to the actual risk of developing the disease. The Göttingen Research Association for Schizophrenia (GRAS) data collection enables us to follow this hypothesis(Begemann et al, 2010; Ribbe et al, 2010). With $>1000$ comprehensively phenotyped schizophrenic patients and $>3000$ data points per subject, the GRAS data collection is an exceptional basis to study genetic causes of or contributions to the schizophrenic phenotype in a 'phenotype-based genetic association study (PGAS)'. This approach is different from and complementary to the genome-wide association studies (GWAS) on schizophrenia as a disease. Rather than searching for 'schizophrenia risk genes', we explore the contribution of genetic variants of candidate genes to schizophrenia-relevant phenotypes.

We show here for the first time that a long CAG repeat length in the KCNN3 gene leads to an electrophysiologically detectable reduction in SK3 conductance. Importantly, long repeat lengths are associated with better cognitive performance of schizophrenic patients in tasks involving the capacity to discriminate, select and execute. Increased SK3 channel activity in turn, as modelled by Kcnn 3 overexpression in mice, selectively leads to remarkable deficits in a comparable set of higher brain functions.

\section{RESULTS}

Case-control study: the CAG repeat length in the KCNN3 gene is not associated with schizophrenia

The CAG repeat polymorphism in the KCNN3 exon 1 coding region has been described in several primate species (Fig $1 \mathrm{~A}$ and B). We first conducted a case-control study to explore a potential role of the KCNN3 CAG repeat lengths sum of both alleles as a genetic risk factor for schizophrenia. No significant difference in the distribution of repeat lengths sum between cases $(n=1060)$ 
A

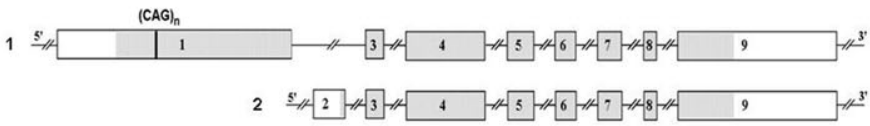

C

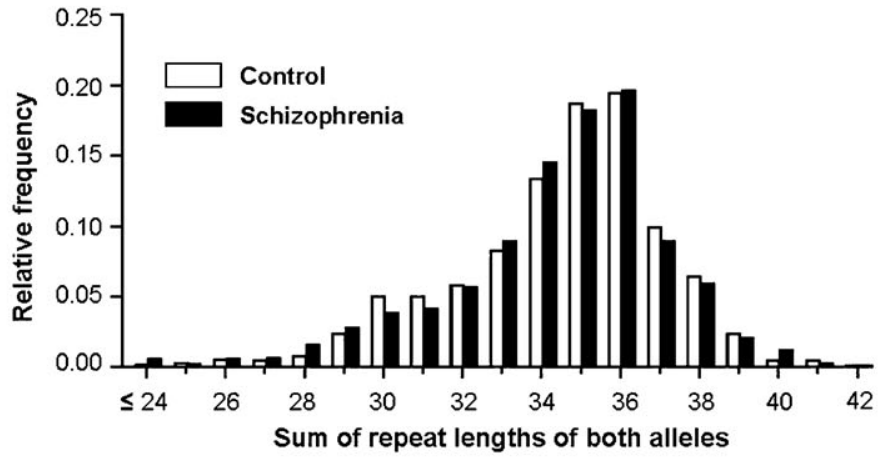

E

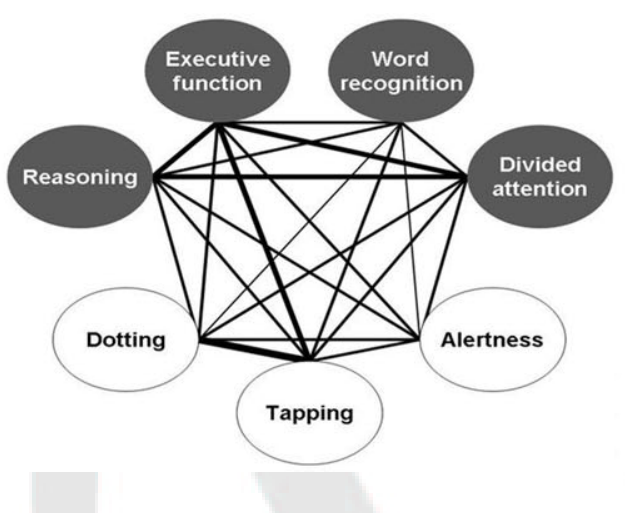

B

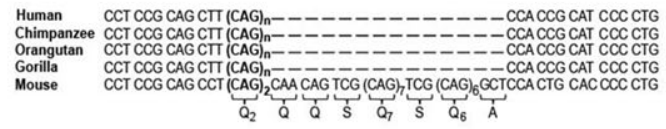

D

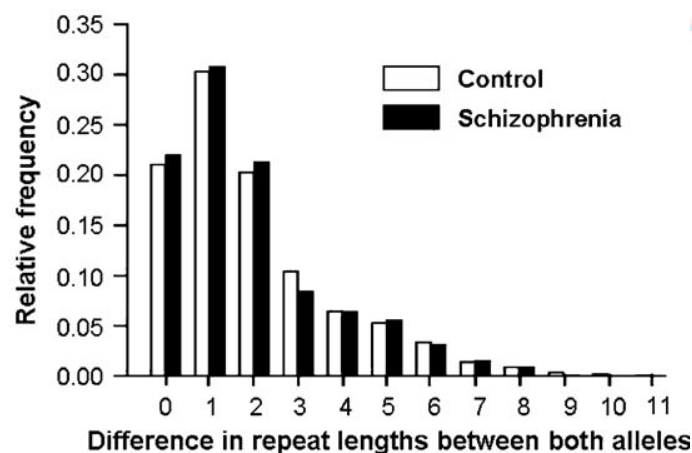

Difference in repeat lengths between both alleles

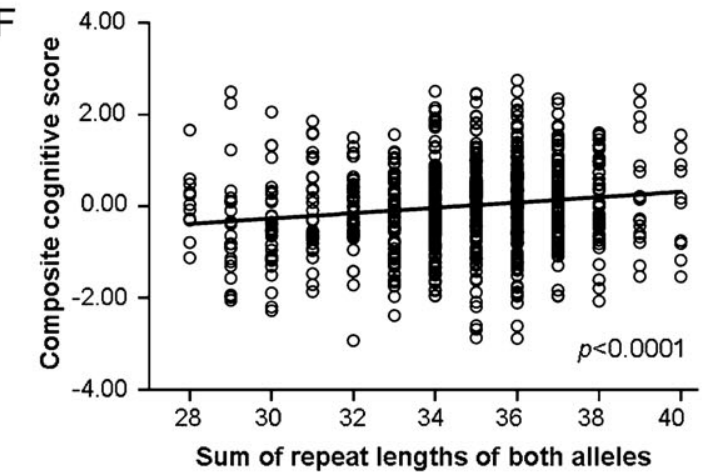

Figure 1. SK3 CAG repeat lengths sum is associated with higher cognitive function in schizophrenia but does not constitute a genetic risk factor for the disease.

A. KCNN3 is located at $1 q 21.3$ and spans $162.8 \mathrm{kbp}$. The nine exons (boxes) encode two different splicing variants (1, 2); the coding region is shaded in grey. The black line in exon 1 indicates the position of the polymorphic CAG repeat.

B. The region around the CAG repeat is highly conserved among species.

C,D. Neither the distribution of the individual sum of repeat lengths of both alleles (C) nor that of the individual difference between repeat lengths of both alleles (D) is different between schizophrenic patients $(n=1060)$ and healthy controls $(n=1135)$. Hence, these readouts of the SK3 CAC repeat polymorphism do not support a genetic risk for developing schizophrenia.

E,F. In contrast, the PGAS approach allows identification of a role for the SK3 CAC repeat polymorphism in higher cognitive function.

E. Intercorrelation network of cognitive target variables (dark ovals) and cognitive control variables (light ovals) in the GRAS population of schizophrenic patients. Line thickness indicates the degree of correlation between two respective tests after standardization by Blom transformation and adjustment for covariates sex, age, antipsychotic medication and negative symptoms.

F. Scatter plot of the covariate-adjusted composite score calculated as mean of all standardized (Blom transformed) cognitive target variables. Adjusted was for covariates sex, age, antipsychotic medication and negative symptoms. Linear regression analysis reveals a significant effect $(p<0.0001)$ of allelic repeat lengths sum on the composite score.

and healthy controls ( $n=1135$ ) was found (Fig 1 C; $\chi^{2}=5.69$, $p=0.82$, evaluated with Monte Carlo sampling on 1000 runs; for details see Supporting information). Also, no gender influence was observed. An association analysis of single allele repeat lengths instead of allelic repeat lengths sum between cases and controls did not yield significant distribution differences either (data not shown). Furthermore, the intraindividual difference of repeat lengths as a measure of marker heterogeneity did not vary significantly between cases and controls (Fig 1D; $\chi^{2}=4.12$, $p=0.65$, 1000 Monte Carlo simulations). Thus, as assumed, there is no evidence for a role of the SK3 CAG repeat length in the risk to develop schizophrenia.
Phenotype-based genetic association study: the KCNN3 CAG repeat length is associated with higher cognition in schizophrenia

Since the essence of the PGAS approach is not to identify potential risk genes for schizophrenia but to understand the contribution of a particular genotype to normal and to disease phenotypes, we moved on to investigate the impact of KCNN3 on core phenotypes of schizophrenia, i.e. cognition, positive and negative symptoms. Following our hypothesis that SK3 may influence higher cognitive functions, we analysed the CAG repeat polymorphism of the $K C N N 3$ gene with respect to neuropsychological test performance of the GRAS sample of schizophrenic patients. We first constructed 
an intercorrelation network, comprising readouts of higher cognition (encompassing capabilities of discrimination, selection and execution) as target variables, and tests of motor-dependent basic cognitive functions as cognitive control variables (Fig 1E). Target variables included reasoning (LPS3), executive function (TMT-B), word recognition (VLMT) and divided attention (TAP). The target variables were internally consistent (Cronbach's $\alpha=0.703$ ), and used for the multivariate models. Cognitive control variables were alertness (TAP), dotting and tapping, all correlating with the cognitive target variables but likely differently regulated. To investigate the influence on general intelligence, a test for premorbid intelligence (MWT-B) was added as additional cognitive control variable. In addition to cognition, PANSS positive and PANSS negative symptoms were analysed as disease-related control variables. Body length served as disease-unrelated control variable (for a detailed description of all variables see Supporting information). Sample characteristics regarding sociodemographic and clinical variables are displayed in Table 1.

The distribution of allelic repeat lengths sum and allelic repeat lengths difference in the schizophrenic population is displayed in Table S1. Only the allelic repeat lengths sum proved to be a genetic readout associated with our phenotypes of interest. The influence of the sum of the repeat lengths of both alleles was studied (i) by a multivariate regression model considering the respective target or control phenotypes as correlated outcomes (Table 2) and (ii) by a classification model (Table S2). Additionally, uncorrected raw data is presented in Table S3. The slope of the regression model estimates the change of covariate-adjusted mean phenotype value per additional repeat in the sum of repeat lengths of the two alleles. The classification model estimates the difference in the covariate-adjusted phenotype means between groups with low (below-median; <35) and high (above-median; >35) repeat lengths sum. The results of the regression and the classification model agree well. Both models show consistently that higher sums of CAG repeats are associated with higher scores (better performance) in the cognitive target but not the cognitive control variables including premorbid intelligence, representing the development-dependent intellectual state at disease onset. Also, PANSS positive, PANSS negative and disease-unrelated control variables are not influenced by repeat sums. Additional individual univariate analyses confirmed that three out of four single target variables included in the multivariate analysis are significantly associated with CAG repeat lengths sum, while this is not the case for any single control variable. For illustration, the influence of the repeat lengths sum on the composite cognitive phenotype is displayed as a simple regression line in Fig $1 F$. To conclude, the PGAS approach reveals a role of the SK3 CAG repeat lengths for higher cognition, best characterized as the cognitive steps 'discriminate, select and execute', but not for psychopathological symptoms of schizophrenia.

\section{Translational approach: SK3 overexpressing mice show selective impairment in higher cognitive function}

Based on above findings and earlier data indicating that SK3 negatively regulates cognition (Blank et al, 2003), we hypothesized that a long CAG repeat length would result in a less functional SK3 channel and better cognition, while a short repeat length would lead to more efficient SK3 function and worse cognitive performance. To test this hypothesis, we analysed basic behaviour and cognition in a transgenic mouse line in which the murine SK3 gene is overexpressed under control of its own regulatory elements, enhanced in cis by the tetracyclin-dependent transactivator (tTA) in the absence of any doxycyclin (Bond et al, 2000). This SK3 overexpressing allele is referred to as SK3-T in the following. The overexpression of SK3

\begin{tabular}{|c|c|c|c|c|}
\hline & $\begin{array}{l}\text { Total GRAS sample } \\
\qquad(n=1060)\end{array}$ & $\begin{array}{l}\text { Low allelic repeat sum }{ }^{a} \\
\qquad(n=462)\end{array}$ & $\begin{array}{l}\text { High allelic repeat sum }{ }^{a} \\
\qquad(n=405)\end{array}$ & $p^{\mathbf{b}}$ \\
\hline \multicolumn{5}{|l|}{ Sociodemographic variables } \\
\hline Age, mean \pm SD (range), $y$ & $39.67 \pm 12.75(18-83)$ & $39.37 \pm 13.04(18-78)$ & $39.31 \pm 12.18(18-73)$ & 0.765 \\
\hline Gender, No. (\%), male & $707(66.7 \%)$ & $301(65.2 \%)$ & $275(67.9 \%)$ & 0.392 \\
\hline Ethnicity, No. (\%), caucasian & $1008(95.6 \%)$ & $437(94.8 \%)$ & $387(96.3 \%)$ & 0.064 \\
\hline Years of education ${ }^{c}$, mean $\pm S D$ (range) & $12.04 \pm 3.05(8-27)$ & $11.92 \pm 2.91(8-24)$ & $12.15 \pm 3.11(8-24)$ & 0.528 \\
\hline Inpatients at assessment, No. (\%) & $449(42.6 \%)$ & $192(41.7 \%)$ & $167(41.5 \%)$ & 0.869 \\
\hline \multicolumn{5}{|l|}{ Clinical variables } \\
\hline Age at 1 st episode, mean \pm SD (range), $y$ & $26.46 \pm 9.02(5.40-73.86)$ & $25.95 \pm 9.00(5.40-73.86)$ & $26.41 \pm 8.74(7.95-57.35)$ & 0.423 \\
\hline $\mathrm{CPZ}$, mean $\pm \mathrm{SD}$ (range) & $683.83 \pm 700.89(0-7500.00)$ & $669.70 \pm 691.73(0-6837.43)$ & $710.66 \pm 769.41(0-7500.00)$ & 0.875 \\
\hline Diagnosis, No. (\%), schizophrenia ${ }^{d}$ & $785(74.5 \%)$ & $339(73.5 \%)$ & $298(74.1 \%)$ & 0.972 \\
\hline Numbers of hospitalizations, mean \pm SD (range) & $8.56 \pm 9.81(0-97)$ & $8.41 \pm 9.46(0-97)$ & $8.74 \pm 10.43(0-82)$ & 0.756 \\
\hline PANSS pos, mean \pm SD (range) & $1.94 \pm 0.89(1-5.43)$ & $1.91 \pm 0.86(1-5.00)$ & $1.95 \pm 0.90(1-5.43)$ & 0.558 \\
\hline PANSS neg, mean \pm SD (range) & $2.60 \pm 1.13(1-6.57)$ & $2.56 \pm 1.07(1-5.71)$ & $2.59 \pm 1.17(1-6.29)$ & 0.988 \\
\hline PANSS gen, mean \pm SD (range) & $2.10 \pm 0.74(1-5.13)$ & $2.09 \pm 0.71(1-4.88)$ & $2.07 \pm 0.76(1-4.69)$ & 0.423 \\
\hline $\mathrm{GAF}$, mean $\pm \mathrm{SD}$ (range) & $45.91 \pm 17.31(5-90)$ & $46.25 \pm 17.13(8-90)$ & $45.41 \pm 17.26(5-90)$ & 0.441 \\
\hline $\mathrm{CGI}$, mean $\pm \mathrm{SD}$ (range) & $5.55 \pm 1.09(2-8)$ & $5.55 \pm 1.07(2-8)$ & $5.56 \pm 1.11(2-8)$ & 0.688 \\
\hline
\end{tabular}

a Low (below-median; <35) and high (above median; >35) allelic repeat lengths sum; individuals with an allelic repeat lengths sum of 35 were excluded ( $n=193$ ).

bstatistical methods used: Mann-Whitney- $U$ or $\chi^{2}$-tests.

${ }^{c}$ Rating according to graduation/certificate; patients currently in school or in educational training are excluded.

${ }^{\mathrm{d} V e r s u s ~ s c h i z o a f f e c t i v e ~ d i s o r d e r s ~ a n d ~ o t h e r ~ p s y c h o t i c ~ d i s o r d e r s / y e t ~ t o ~ b e ~ c o n f i r m e d . ~}$ 


\begin{tabular}{|c|c|c|c|c|}
\hline \multirow[t]{3}{*}{ Phenotypes } & \multicolumn{2}{|c|}{ Change of mean phenotype value } & \multirow{2}{*}{\multicolumn{2}{|c|}{ Statistical test }} \\
\hline & & nal repeat & & \\
\hline & estimate & $95 \%$ confidence interval & $t$-value (1df) & $p$-value \\
\hline \multicolumn{5}{|l|}{ Cognitive target variables: } \\
\hline Combined (multivariate) & 0.0269 & $0.0104,0.0434$ & 3.1901 & $0.0014^{a}$ \\
\hline \multicolumn{5}{|l|}{ Individual (univariate) } \\
\hline Reasoning & 0.0224 & $0.0008,0.0440$ & 2.0317 & $0.0425^{\mathrm{b}}$ \\
\hline Executive function & 0.0281 & $0.0062,0.0500$ & 2.5203 & $0.0119^{b}$ \\
\hline Word recognition & 0.0331 & $0.0096,0.0565$ & 2.7680 & $0.0058^{b}$ \\
\hline Divided attention & 0.0319 & $0.0069,0.0570$ & 2.5004 & $0.0126^{b}$ \\
\hline \multicolumn{5}{|l|}{ Cognitive control variables: } \\
\hline Combined (multivariate) & 0.0132 & $-0.0039,0.0304$ & 1.5105 & $0.1310^{\mathrm{a}}$ \\
\hline \multicolumn{5}{|l|}{ Individual (univariate) } \\
\hline Dotting & 0.0163 & $-0.0059,0.0385$ & 1.4391 & 0.1505 \\
\hline Tapping & 0.0142 & $-0.0079,0.0363$ & 1.2612 & 0.2076 \\
\hline Alertness & 0.0115 & $-0.0105,0.0335$ & 1.0281 & 0.3042 \\
\hline Premorbid intelligence & 0.0088 & $-0.0155,0.0330$ & 0.7090 & $0.4785^{\mathrm{a}}$ \\
\hline \multicolumn{5}{|l|}{ Disease-related control variables: } \\
\hline PANSS positive symptoms & 0.0051 & $-0.0186,0.0288$ & 0.4230 & $0.6724^{\mathrm{a}}$ \\
\hline PANSS negative symptoms & 0.0131 & $-0.0120,0.0382$ & 1.0236 & $0.3063^{\mathrm{a}}$ \\
\hline \multicolumn{5}{|l|}{ Disease-unrelated control variable: } \\
\hline Body length & 0.0151 & $-0.0042,0.0344$ & 1.5365 & $0.1248^{a}$ \\
\hline
\end{tabular}

${ }^{a, b}$ Multiple testing corrected significance thresholds: ${ }^{a}$ first level of tests $p \leq 0.0100$ (Bonferroni), ${ }^{b}$ second level of tests $p \leq 0.0138$ (permutation test with 50000 permutations to account for correlations between phenotypes).

Association analyses of allelic repeat lengths sum with mean value of target and control phenotypes. The analysed sample has a range of allelic repeat lengths sums from 28 to 40,n=952. Phenotypes were adjusted for sex and age, additionally for medication (all cognitive phenotypes, PANSS positive and PANSS negative symptoms) and for negative symptoms (all cognitive phenotypes). All phenotypes were standardized to zero mean and variance one: larger values for cognitive phenotypes correspond to better performance. Like Cohen's $d$, the genetically induced effect size (change of mean phenotype value per additional repeat) is quantified relative to the standard deviation of the trait. Rare extreme observations of allelic repeat lengths sums (below 28 , above $40 ; n=21$ ) and non-native German speakers with language problems (total of $n=87$ ) were excluded from the analyses (to result in $n: 1060-108=952$ ).

in this model is illustrated in Fig $2 \mathrm{~A}$ and $\mathrm{B}$, comparing hippocampal SK3 immunoreactivity in a wildtype and a SK3 T/T mouse. Densitometric analysis of the SK3 specific bands obtained by Western blot demonstrates the pronounced increase in SK3 protein in hippocampus $(p=0.0029)$ and heart (used as a control tissue; $p=0.0005$ ) of SK3 T/T mice (Fig 2C).

Most basic behavioural tests were unaffected by SK3 overexpression: Time spent in open arms of elevated plus maze (Fig 2D) as well as time spent in the different zones and velocity in the open field (Fig 2E and F) were unchanged, indicating comparable anxiety and activity levels in both experimental groups. However, SK3 T/T mice showed an increase in latency to reach the wall when first put in the open field ( $p=0.029$; Fig $2 \mathrm{G}$ ). Exploratory activity in the hole board test was reduced in mice overexpressing SK3 ( $p=0.0004$; Fig $2 \mathrm{H}$ ). Importantly, motor performance and motor learning, as evaluated in the 2-day rotarod testing, were comparable in both genotypes (Fig 2I). No differences between genotypes were found in sucrose preference, pre-pulse inhibition, and social interaction tests (Fig S1A-D).

In the visible platform training ( 2 days) of the Morris water maze, there was no overall difference between SK3 T/T and WT mice. Both experimental groups had similar latency to locate the visible platform (2-way repeated measurement ANOVA: effect of genotype $F_{1,26}=0.23, p=0.637$ ), similar swim distance to the platform (2-way repeated measurement ANOVA: effect of genotype $F_{1,26}=0.96, p=0.337$ ), and similar swim velocity (2-way repeated measurement ANOVA: effect of genotype $F_{1,26}=1.09, p=0.306$ ). Notably, however, in the hidden platform testing, assessing the ability to use extra-maze spatial cues to locate a submerged platform, impaired spatial learning in SK3 overexpressing mice became evident, with longer latencies to find the platform over the trials (2-way repeated measurement ANOVA: effect of genotype $F_{1,26}=5.43, p=0.028$, Fig $2 \mathrm{~J}$ ) as well as longer swim distance to the platform (2-way repeated measurement ANOVA: effect of genotype $F_{1,26}=11.17, p=0.0025$; not shown). Moreover, in the probe trial, performed to determine whether mice use a spatial strategy to find the platform, SK3 T/T mice spent less time in the target quadrant ( $p=0.033$; Fig $2 \mathrm{~J}$, inset and Fig S1E). Along the same lines, SK3 overexpressing mice displayed reduced freezing behaviour in fear conditioning when tested $72 \mathrm{~h}$ after training in the same context $(p=0.036$; Fig $2 \mathrm{~K})$, reflecting compromised hippocampal memory. Significant differences between groups were also observed in the cued memory test with SK3 T/T mice showing a decreased freezing response to the conditioned tone ( $p=0.0008$; Fig $2 \mathrm{~K}$ ). Taken together, overexpression of SK3, similar to the short CAG repeat length genotype in humans, leads to reduced performance in higher brain functions.

\section{Mechanistic insight: SK3 channel conductance depends on CAG repeat length}

To test if the CAG repeat length indeed has an impact on channel function, we expressed three different human SK3 isoforms, 

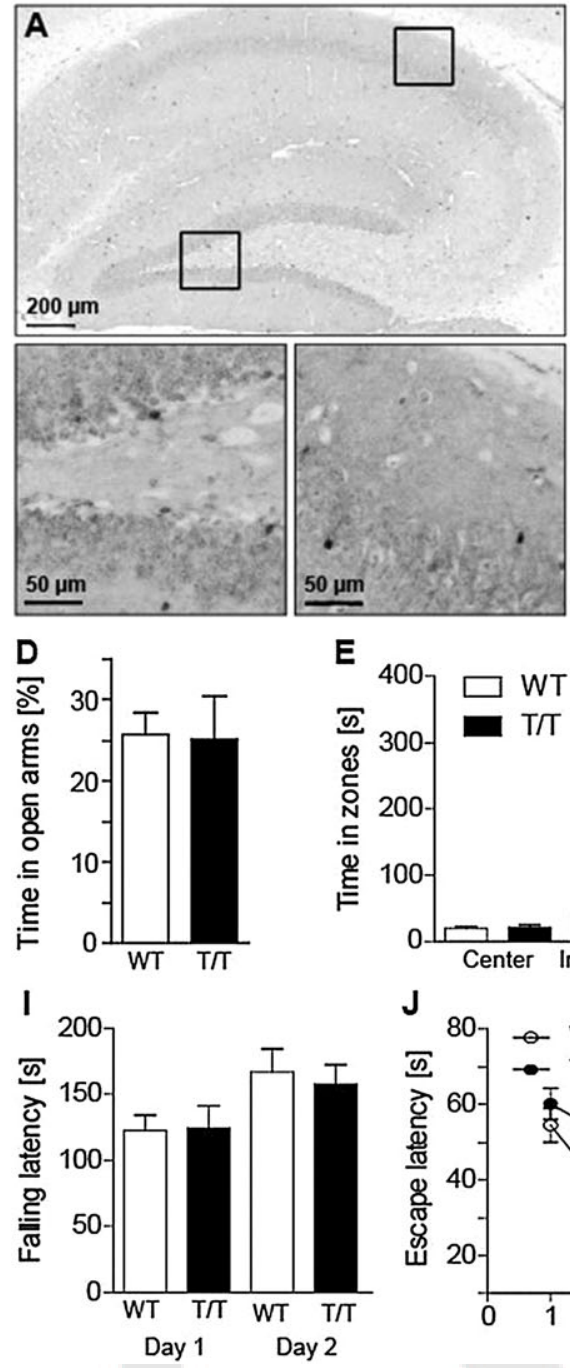
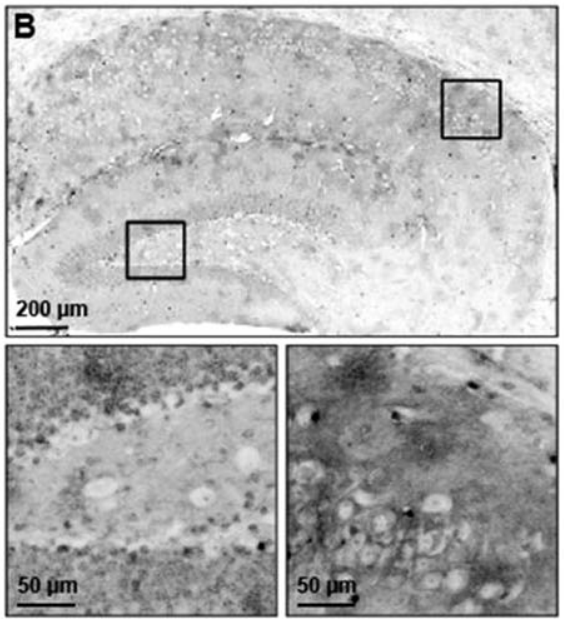

$\mathbf{F}$

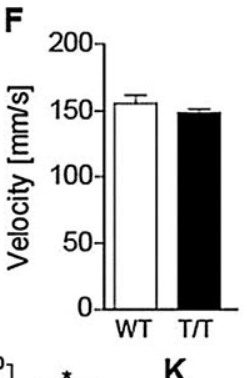

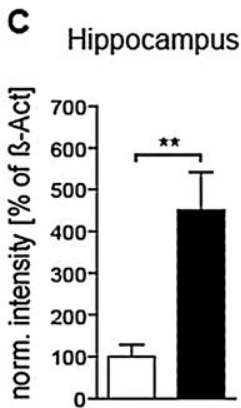

Heart
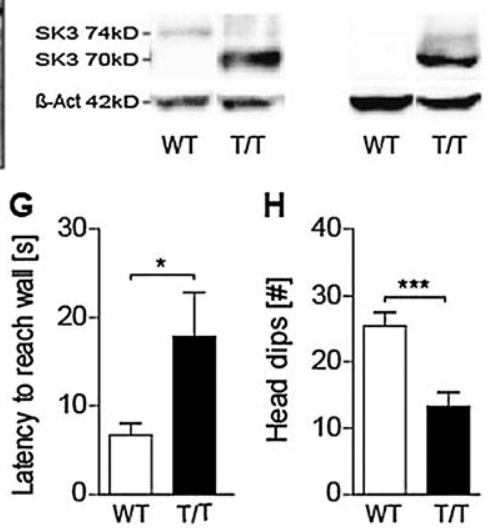
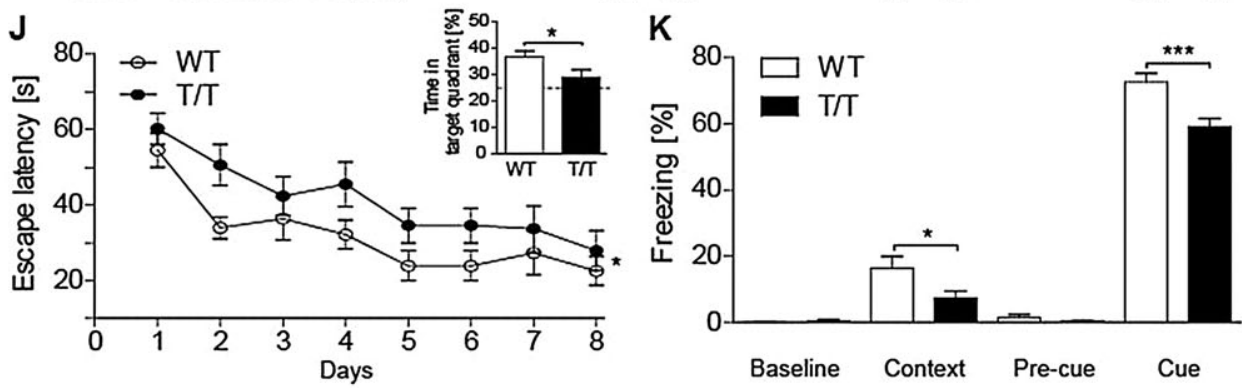

Figure 2. SK3 overexpressing (T/T) mice show selective deficits in higher cognition.

A,B. Comparative distribution of SK3 immunoreactivity in hippocampus (sagittal section) is presented for wildtype (A) versus SK3 T/T mice (B). Insets show magnifications of stained granule neurons in dentate gyrus and pyramidal neurons in Ammon's horn (CA2).

C. Densitometrical quantification of SK3 protein expression in hippocampus and heart of WT and SK3 T/T mice ( $n=6-7$; ${ }^{* *}$ Student's $t$-test, $t_{1,12}=3.724$, $p=0.0029 ;{ }^{* * *}$ Student's $t$-test, $\left.t_{1,11}=4.902, p=0.0005\right)$. SK3 expression is presented as \% of $ß$-actin (ß-Act) and normalized to the WT. Sample Western blots are shown below the bar chart. For quantification, the $74 \mathrm{kDa}$ band was used for WT (endogeneous SK3) and the $70 \mathrm{kDa}$ band for T/T (transgenic SK3 expression).

D. WT and T/T behaviour is unaltered in elevated plus maze. Presented is the percentage of time spent in open arms against the total time spent in both open and closed arms.

E-G. No differences in open field are observed between genotypes regarding time spent in different zones (E) or velocity (F). The latency to reach the wall after initial start from the centre of the open field (G) is significantly higher in SK3 T/T mice compared to WT ("Student's $t$-test, $t_{1,26}=2.318, p=0.029$ ).

H. In hole board, SK3 T/T mice show significantly less exploratory activity than WT (***Student's $t$-test, $\left.t_{1,26}=4.110, p=0.0004\right)$.

I. The two genotypes do not differ in motor performance/motor learning on rota-rod.

J. SK3 T/T mice demonstrate longer escape latency in the hidden platform task ( ${ }^{*}$ two-way repeated measures ANOVA, $\left.F_{1,26}=5.43, p=0.028\right)$; in the probe trial (inset), SK3 T/T mice display absence of preference for the target quadrant ('Student's $t$-test, $t_{1,26}=2.250, p=0.033$ ).

K. In contextual and cue memory test of fear conditioning, SK3 T/T mice freeze less ( ${ }^{*}$ Student's $t$-test, $t_{1,26}=2.212, p=0.036,{ }^{* * *}$ Student's $t$-test, $t_{1,26}=3.779$, $p=0.0008$ ). For all behavioural experiments: $n=13-15$; data presented as mean \pm s.e.m.; two-sided Student's $t$-tests used; ${ }^{*}$ indicates $p<0.05$, ${ }^{* *} p<0.01$ and ${ }^{* * * *} p<0.001$.

characterized by different repeat lengths and fused with eGFP, in HEK293 cells and performed whole-cell patch clamping (Fig 3). Cells transfected with the constructs $\left(\text { eGFPhSK3 }(\mathrm{CAG})_{11} \text {, eGFPhSK3(CAG) }\right)_{18}$, eGFPhSK3(CAG) 24$)$ showed intense fluorescence signal compatible with cell surface expression (Fig 3A and B). No obvious localization or quantitative differences among clones were observed under epifluorescence microscopy, and the size of the expressed 


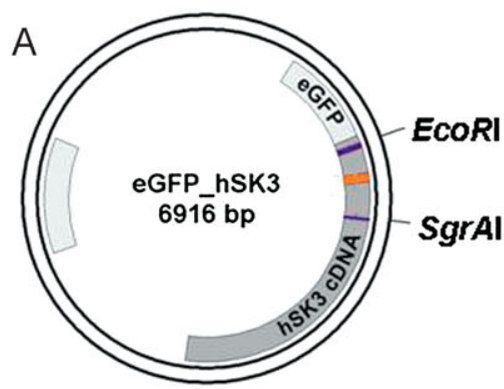

B

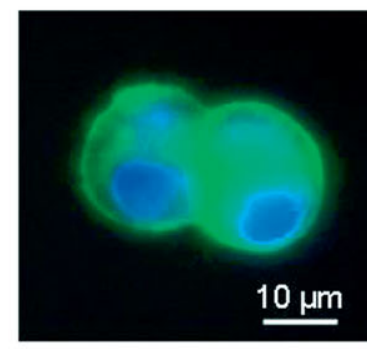

C

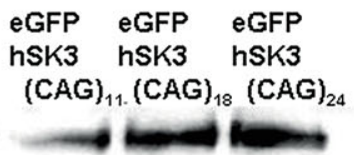

eGFP/SK3

$\alpha-$ tubulin
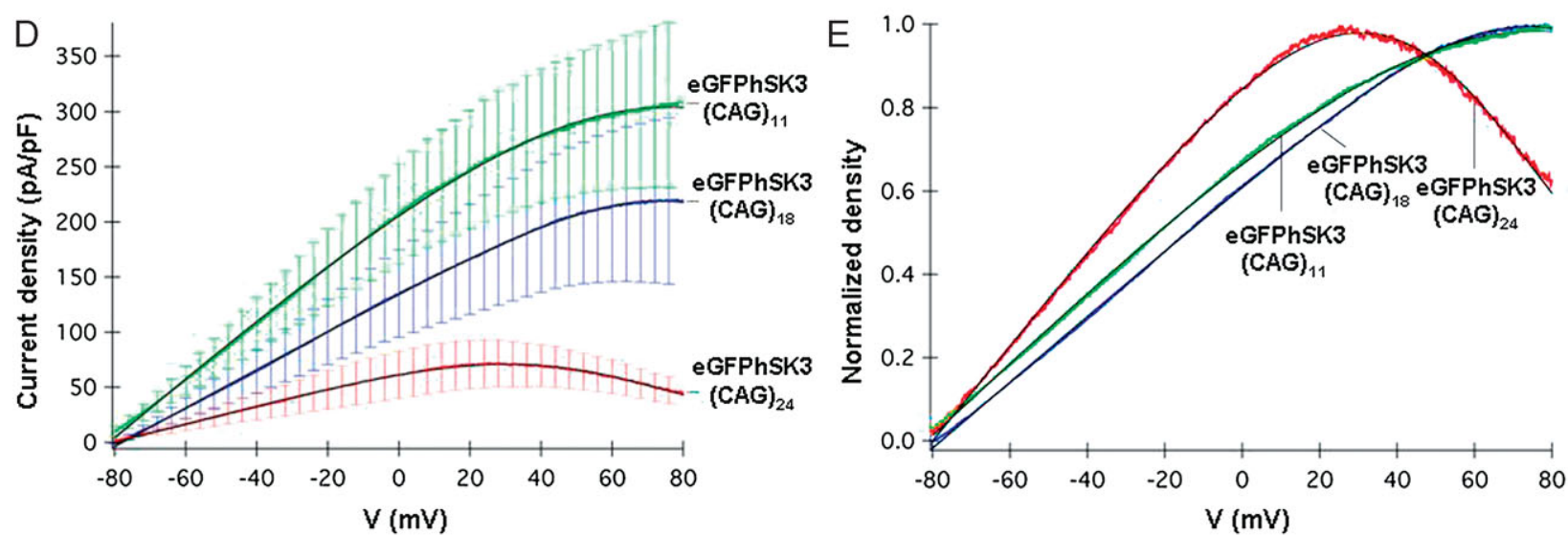

Figure 3. KCNN3 CAG repeat length affects electrophysiological properties of SK3.

A. For electrophysiological measurements, the eGFPSK3(CAG) $)_{n}$ construct has been employed to generate clones with different repeat lengths. Indicated are locations of the CAC repeat (labelled in orange), of the primers (labelled in purple) and of the restriction enzyme sites used for cloning.

B. Live transfected HEK293 cells show intense green fluorescent signal on the plasma membrane; cell nuclei are stained with Hoechst33342 (blue).

C. Western blot analysis of eGFP and SK3 demonstrates comparable SK3 fusion protein size and expression level of the different constructs in transfected cells.

D. The average SK3 current response (expressed as apamin-sensitive current density) for eGFPhSK3(CAG) 11 ( $n=8$; green), eGFPhSK3(CAG) 18 ( $n=7$; blue) and eGFPhSK3(CAG) $_{24}(n=9$; red) is displayed. The result of fitting is superimposed as a solid line. Error bars represent s.e.m.

E. Normalized apamin-sensitive current density (versus the corresponding individual current density maximum) for clones eGFPhSK3(CAG) ${ }_{11}$, eGFPhSK3(CAG) 18 and eGFPhSK3(CAG) 24 . Inward rectification starting as early as at $+20 \mathrm{mV}$ is evident for eGFPhSK3(CAG) 24 . Colours as in panel $\mathrm{D}$.

proteins was comparable as predicted (Fig 3C), excluding truncation artifacts. Measurement of apamin-sensitive current density showed a significant reduction in eGFPhSK3(CAG) 24 as compared to eGFPhSK3(CAG) ${ }_{18}$ and eGFPhSK3(CAG) ${ }_{11}$. Overall conductance $( \pm 95 \%$ confidence interval) calculated was $0.83 \pm 0.002(n=9), \quad 1.75 \pm 0.002(n=7)$ and $2.72 \pm 0.011$ $(n=8) \mathrm{pA} / \mathrm{pF}$, respectively (Fig $3 \mathrm{D})$. The shape of the current/voltage relationship for SK3 channels is strongly inwardly rectifying (Grunnet et al, 2001). We observed a clear rectification in cells expressing eGFPhSK3(CAG) 24 already at less depolarized potentials than in eGFPhSK3(CAG) ${ }_{11}$ and eGFPhSK3(CAG) 18 . Plotting the average normalized current against voltage, a gradual increase in the degree of rectification between the different isoforms was observed. Voltage for halfmaximal block was $117.22 \pm 0.395,113.89 \pm 0.402$ and $61.251 \pm 0.136 \mathrm{mV}$ for eGFPhSK3 (CAG) $)_{11}$, eGFPhSK3(CAG) 18 and eGFPhSK3(CAG) 24 , respectively (Fig 3E). Qualitatively identical results were obtained in control experiments with an untagged expression of hSK3 clones (Fig S2). Overall, these data support our hypothesis that a long CAG repeat length reduces SK3 channel conductance.

\section{DISCUSSION}

We report here the surprising finding that a normal variant of the gene encoding the small conductance calcium-activated potassium channel SK3 predicts cognitive performance of patients with schizophrenia. The discovery of KCNN3 CAG repeat lengths influencing higher cognition in man was facilitated by a PGAS on the grounds of our new schizophrenia patient database, the GRAS data collection.

The role of simple sequence repeats as genetic modulators of brain function and behaviour is quite well established, however, in most cases the biological mechanisms involved are far from clear (Fondon et al, 2008). Notably, the present study allows mechanistic insight, elucidating the functional role of the polymorphic glutamine repeats in the $N$-terminal coding region of SK3. Although the $N$-terminus of the channel does not directly contribute to the core structure of the pore, it may, as in many other channels, form part of the internal vestibule and thereby influence permeation properties. As documented here by wholecell patch-clamping, long CAG repeats reduce current amplitude at depolarized potentials in the presence of internal $\mathrm{Ca}^{2+}$. This 
reduced channel conductance is associated in schizophrenic patients with better performance in higher cognitive tasks, comprising the capabilities to 'discriminate, select and execute'. On the contrary, SK3 overexpression in mice, as a model of increased channel function, leads to reduced cognitive abilities in a similar set of tasks. Thus, SK3 conductance appears to be inversely correlated with higher cognition.

The electrophysiological results obtained here with transfected cells help to explain this conclusion which is somewhat unexpected at first glance. Our data indicate an enhanced $\mathrm{Ca}^{2+}$ block in the SK3 isoform with the longest repeats as compared to the shorter repeat variants, leading to an overall decreased conductance at a given $\mathrm{Ca}^{2+}$ concentration in a physiologically relevant range. This would generate different after hyperpolarization depending on the length of the repeat, and ultimately result in increased excitability, as shown for dopaminergic neurons, where decreasing the activity of SK channels by decreasing the apparent $\mathrm{Ca}^{2+}$ affinity changes the firing pattern from a pacemaker to an irregular or bursting one (Ji et al, 2009). The phenotypic effect of different CAG repeat or polyglutamine lengths can also be independent of the electrophysiological properties of the resulting channel, since it might alter the affinity of RNA binding proteins and/or protein-protein interactions (Jasinska et al, 2003; Orr \& Zoghbi, 2007).

A 'less is more' feature does not seem to be entirely unusual for potassium channels influencing cognitive readouts. Along these lines, apamin treated mice show superior cognitive behaviour, while overexpression of SK2 specifically leads to cognitive impairment in similar tasks (Hammond et al, 2006; Stackman et al, 2002). Also, disruption of the gene encoding another type of potassium channel, the voltage-gated channel $\mathrm{BEC} 1 / \mathrm{KCNH} 3$, changes hippocampal neuronal activity as well as synaptic plasticity, and enhances cognitive function in mice (Miyake et al, 2009). A primate-specific isoform of yet another member of the voltage-gated ether-à-go-go family, KCNH2 (ERG1), is highly expressed in post mortem brains of schizophrenics. This shorter isoform appears to be inversely correlated with cognition and to impair channel function (Huffaker et al, 2009).

In our study, focusing on the CAG repeat polymorphism in $K C N N 3$, the gene encoding SK3, a small conductance calciumactivated potassium channel, the distribution of repeat lengths was comparable in both schizophrenic and healthy subjects. This finding confirms the meta-analysis by Glatt et al (2003) and points against a major role of this particular genetic marker for the risk to develop schizophrenia. Also, the present study did not substantiate an effect of glutamine repeat length on positive or negative symptoms as reported for Jewish (Ritsner et al, 2002) or British schizophrenic patients (Cardno et al, 1999). This discrepancy may be explained by ethnical differences or by a potential bias due to the small sample sizes used in these earlier studies (Cardno et al, 1999; Ritsner et al, 2002). In agreement with the present findings on cognitive readouts in schizophrenic patients, our results obtained with the Kcnn3 overexpressing mice further support a role of this gene in higher cognition, whereas readouts of positive symptoms (e.g. hyperactivity) or negative symptoms (e.g. social interaction, sucrose preference) were not seen in the SK3 overexpressing as compared to WT mice (Fig S1).

The observation that long glutamine repeats, and thus reduced SK3 channel function, are equally disseminated in healthy and schizophrenic subjects makes it very likely that the "long repeat SK3 effect' on higher cognition is not restricted to schizophrenia, although functional proof in healthy individuals and in other disease cohorts is still required. Nevertheless, the SK3 genotype clearly contributes to the cognitive phenotype of schizophrenic patients. Even if traits of interest in schizophrenia (here: cognition) are never explained by a single modifier gene only, candidate alleles like the SK3 variants shown here codetermine, together with other trait-relevant genes and environmental factors, the outcome of an individual suffering from schizophrenia.

In fact, we hypothesize that an interplay of multiple causative factors, perhaps thousands of potential combinations of genes/ genetic markers and an array of different environmental risks, leads to the development of a schizophrenic phenotype. Not too much of an overlap may exist between genetic risk factors from one schizophrenic patient to an unrelated other schizophrenic individual, explaining why it is basically impossible to find common risk genes of schizophrenia with appreciable odds ratios. In the overwhelming majority of cases, schizophrenia seems to be the result of a 'combination of many unfortunate genotypes', and a short SK3 repeat variant may be one of them.

Given the obvious influence of SK3 conductance on higher cognition, this channel may be an interesting pharmacological target for addressing cognitive function in disease conditions associated with cognitive deficits.

\section{MATERIALS AND METHODS}

For a more comprehensive version of Materials and methods see Supporting information.

\section{Human study}

\section{Subjects}

The present study has been based on the GRAS data collection of $n=1074$ patients (as of October 2009) diagnosed according to DSMIV-TR with schizophrenia (73.2\%), schizoaffective disorder (14.8\%) or other psychotic disorders/yet to be conrmed (12.0\%). Control subjects were healthy blood donors $(n=1143)$. The GRAS data collection as well as the healthy control population is described in greater detail elsewhere (Begemann et al, 2010; Ribbe et al, 2010).

\section{Genotyping}

Standard methods were used for DNA extraction from whole blood (Genomed $\mathrm{GmbH}$, Löhne, Germany). The polymorphic CAG repeat in exon1 of KCNN3 was amplied from genomic DNA by PCR. Primers were chosen according to Austin et al (1999), resulting in a PCR fragment of 121 bp. The amplicons were separated using size electrophoresis on the ABI 3730 XL DNA Analyser (Applied Biosystems, Foster City, USA). Raw data were processed using the Gene Mapper Software 4.0 (Applied 


\section{The paper explained}

\section{PROBLEM:}

Schizophrenia has a strong genetic component but it is unclear how genetic variants contribute to the disease phenotype. A polyglutamine repeat in a calcium-activated potassium channel, SK3, which is important for synaptic plasticity, has been discussed for some time as potential risk factor for schizophrenia. However, up to now it has remained unclear which domains of the phenotype might be affected and how a 'risk role' of SK3 might translate to the biological level.

\section{RESULTS:}

We report here that SK3 does not increase the general risk for schizophrenia, but that it contributes in a significant way to the cognitive abilities of schizophrenic patients. Specifically, longer polyglutamine stretches are associated with better cognitive performance. On the molecular level, longer stretches result in a reduced conductance of the channel. On the other hand, mice overexpressing this gene (as a model of humans with shorter polyglutamine sequence) perform cognitively worse than their wildtype littermates. The chain of interactions thus reads: Shorter repeats or SK3 overexpression-enhanced channel function-reduced cognitive performance.

\section{IMPACT:}

We conclude that regarding SK3 channel function and cognition, 'less is more'. Therefore, pharmacological reduction of SK3 channel conductance might be an attractive novel strategy to improve cognition in disease states.
Biosystems). Successful genotyping was performed for 1060 patients and 1135 controls (99\%).

\section{Mouse study}

\section{Behaviour}

Male WT and SK3 T/T mice (Bond et al, 2000) (littermates) were started at the age of 4 months on a battery of behavioural tests, performed in the following order: elevated plus maze, open eld, hole board, rota-rod, pre-pulse inhibition, social interaction, sucrose preference, Morris water maze and fear conditioning.

\section{Immunohistochemistry}

SK3 immunostaining was carried out on parafn sections using a rabbit polyclonal anti-SK3 antibody (Alomone, Jerusalem, Israel; dilution 1:120 v/v) and peroxidase labelled polymer anti-rabbit antibody (Envision HRP System/DAB for rabbit primary antibodies, DAKO, Glostrup, Denmark). Immunocomplexes were visualized by diaminobenzidine (DAB; Sigma-Aldrich, Taufkirchen, Germany). Brighteld images were obtained with an Axiovert 200M microscope (Zeiss, Oberkochen, Germany).

\section{Western blot}

Experiments were performed using a rabbit polyclonal anti-SK3 antibody (Alomone, 1:2500), and a mouse monoclonal anti-beta actin antibody (Abcam, Cambridge, UK, 1:10000) for mouse tissues, or a mouse monoclonal anti- $\alpha$-tubulin antibody (Sigma-Aldrich, 1:20000) for HEK293 cells, and appropriate horseradish-peroxidase-conjugated secondary antibodies (sheep anti-mouse IgG 1:10000 and donkey antirabbit IgC 1:10000, GE Healthcare, Munich, Germany, for mouse tissues or anti-rabbit IgG 1:5000 and anti-mouse IgG 1:10000, Sigma-Aldrich, for HEK293 cells). Immunoreactivity was visualized by chemoluminescence (ECL, Millipore, Billerica, USA) and quantied by the Quantity One analysis software (Bio-Rad, Munich, Germany)

\section{In vitro analysis}

\section{Cloning}

The vector containing eGFP-labelled SK3 was kindly provided by H. Wulff (UC Davis). The different lengths of CAG repeats (11, 18 and 24) were amplied by PCR from respective human samples and cloned into the original vector using ECORI and SgrAl restriction sites. The resulting constructs eGFPhSK3(CAG) ${ }_{11}$, eGFPhSK3(CAG) $)_{18}$, eGFPhSK3(CAG) $)_{24}$ were veried by sequencing. For obtaining constructs without eGFP, vectors were sequentially digested with Agel and BspEl.

\section{Transfection}

HEK293 cells were transfected using Lipofectamine 2000 (Invitrogen, Karlsruhe, Germany) following the manufacturer's guidelines. Stable cell pools were obtained by selection with $300 \mu \mathrm{g} / \mathrm{ml} \mathrm{G-418}$ (Invitrogen). Representative uorescence images of living cells were taken under an epiuorescence microscope; nuclei were stained with Hoechst33342 (Invitrogen).

\section{Electrophysiology}

All measurements were performed by a blinded investigator. Macroscopic currents elicited by a $500 \mathrm{~ms}$ voltage ramp from -80 to $+80 \mathrm{mV}$ were recorded in the whole-cell conguration of the patchclamp technique (Hamill et al, 1981). The intracellular solution contained (in $\mathrm{mM}$ ) $160 \mathrm{KCl}, 0.5 \mathrm{MgCl}_{2}, 10 \mathrm{EGTA}, 9.5 \mathrm{CaCl}_{2}$, (free $\mathrm{Ca}^{2+}$ $1.03 \mu \mathrm{M}), \quad 10 \mathrm{Hepes} / \mathrm{KOH} \mathrm{pH}$ 7.35. The control external recording solution contained (in $\mathrm{mM}$ ) $160 \mathrm{NaCl}, 2.5 \mathrm{KCl}, 2 \mathrm{CaCl}_{2}$, $1 \mathrm{MgCl}_{2}$, 8glucose, 10HEPES/NaOH, pH 7.4. Apamin (100 nM)-sensitive currents were determined by off-line subtraction. To determine overall conductance and voltage of half-maximal block, we used a linear current/voltage function with a block at positive voltages. Goodness of $t$ was evaluated by Pearson's $\chi^{2}$, and condence intervals by Student's $t$ distribution. 


\section{Statistical analysis}

\section{Case-control study}

The sum of repeat lengths of both alleles of all individuals was analysed. To account for the degree of heterogeneity between the two alleles (intraindividual heterogeneity), the difference between allelic repeat lengths was also calculated. Maximized $\chi^{2}$ values for distribution of genotypes among schizophrenic and control samples were determined by Monte Carlo tests (using 1000 simulations) with Clump software (Sham \& Curtis, 1995) (http://www.mds.qmw.ac.uk/statgen/dcurtis/ software. html).

\section{PGAS}

Analyses were based on allelic repeat lengths sum. Statistical analyses were carried out with $\mathrm{R}$ (v2.10.0). Data on cognitive tests are presented in a way that higher values always indicate better performance. Nonnative German speakers with language problems $(n=87)$ were excluded. Metric phenotypes were standardized to zero mean and variance one by Blom transformation (Blom, 1958) and analysed by linear models. Multivariate analysis modelled a target phenotype vector, accounting for individual correlation between vector entries. Variables were adjusted for covariates sex and age, for covariate antipsychotic medication dose (all cognitive phenotypes, PANSS positive symptoms, PANSS negative symptoms) and for covariate negative symptoms (all cognitive phenotypes). Multiple testing adjusted signicant thresholds to the $5 \%$-level were determined by Bonferroni-adjustment or by permutation test (50000 permutations, to account for correlations between cognitive phenotypes).

\section{Animal study}

Statistical signicance was evaluated using unpaired Student's t-test and two-way repeated measurement ANOVA including Bonferroni testing where applicable. Signicance level was set to $p<0.05$. Data are represented as mean \pm s.e.m. in gures and text. The data were analysed using Prism4 (GraphPad Software, San Diego, CA, USA).

\section{Author contribution}

SG carried out genetic analyses and the cloning procedure. She was supported by SP and for sample processing and logistics by FB. The human association study was carried out by MFG. BA performed all behavioural analysis of SK3 T/T mice, and SM the expression analysis. LAP conducted the electrophysiology. DM, MFG and HB performed the genetic statistics. MB coordinated and supervised the travelling team of investigators as well as the database staff. KR and HF were pivotal members of the travelling team and supervised the database. JR provided DNA samples from healthy blood donors. HE, K-AN, NB and WS developed the concept of GRAS, and guided the project, data analysis and paper writing. KAR, PF and MM gave input to data analysis and manuscript preparation. HE, SG, MFG and BA wrote the paper. All authors discussed the results, have read and commented on the manuscript, and have seen and approved the final version.

\section{Acknowledgements}

This study was supported by the Max Planck Society and the DFG-Research Center for Molecular Physiology of the Brain (CMBP). We thank J. P. Adelman and C. T. Bond, Vollum Institute, OHSU Portland, OR, for providing SK3 T/T mice and H. Wulff, UC Davis, CA, for providing the eGFP-hSK3 construct. We further thank Swetlana Sperling, Anja Ronnenberg, Kathrin Hannke, Ursula Kutzke, Barbara Scheufler and Sabine Stolpe for their excellent technical work, and the staff of the animal facility at the Max Planck Institute of Experimental Medicine for maintenance of the mouse colony. Dr Marcio Lazzarini is gratefully acknowledged for stimulating discussions. We are indebted to all patients for their participation in the GRAS study, and all collaborating GRAS centres for their support. We are grateful to all colleagues who contributed to the GRAS data collection.

Supporting information is available at EMBO Molecular Medicine online.

The authors declare that they have no conflict of interest.

\section{References}

Austin CP, Holder DJ, Ma L, Mixson LA, Caskey CT (1999) Mapping of hKCa3 to chromosome 1q21 and investigation of linkage of CAG repeat polymorphism to schizophrenia. Mol Psychiatry 4: 261-266

Begemann M, Grube S, Papiol S, Malzahn D, Krampe H, Ribbe K, Friedrichs $\mathrm{H}$ Radyushkin KA, El-Kordi A, Benseler F et al (2010) Modification of cognitive performance in schizophrenia by complexin 2 gene polymorphisms. Arch Gen Psychiatry 67: 879-888

Blank T, Nijholt I, Kye MJ, Radulovic J, Spiess J (2003) Small-conductance, $\mathrm{Ca}^{2+}$-activated $\mathrm{K}^{+}$channel SK3 generates age-related memory and LTP deficits. Nat Neurosci 6: 911-912

Blom G (1958) Statistical Estimates and Transformed Beta Variables, New York, John Wiley and Sons, Inc.

Bond CT, Sprengel R, Bissonnette JM, Kaufmann WA, Pribnow D, Neelands T, Storck T, Baetscher M, Jerecic J, Maylie J et al (2000) Respiration and parturition affected by conditional overexpression of the $\mathrm{Ca}^{2+}$-activated $\mathrm{K}^{+}$ channel subunit, SK3. Science 289: 1942-1946

Cardno AG, Bowen T, Guy CA, Jones LA, McCarthy G, Williams NM, Murphy KC Spurlock G, Gray M, Sanders RD et al (1999) CAG repeat length in the hKCa3 gene and symptom dimensions in schizophrenia. Biol Psychiatry 45: 15921596

Chandy KG, Fantino E, Wittekindt O, Kalman K, Tong LL, Ho TH, Gutman GA, Crocq MA, Ganguli R, Nimgaonkar V et al (1998) Isolation of a novel potassium channel gene hSKCa3 containing a polymorphic CAG repeat: a candidate for schizophrenia and bipolar disorder? Mol Psychiatry 3: 32-37

Curtain R, Sundholm J, Lea R, Ovcaric M, MacMillan J, Griffiths L (2005) Association analysis of a highly polymorphic CAG Repeat in the human potassium channel gene KCNN3 and migraine susceptibility. BMC Med Genet 6: 32

Dror V, Shamir E, Ghanshani S, Kimhi R, Swartz M, Barak Y, Weizman R, Avivi L, Litmanovitch T, Fantino E et al (1999) hKCa3/KCNN3 potassium channel gene: association of longer CAG repeats with schizophrenia in Israeli Ashkenazi Jews, expression in human tissues and localization to chromosome 1q21. Mol Psychiatry 4: 254-260

Faber ES (2009) Functions and modulation of neuronal SK channels. Cell Biochem Biophys 55: 127-139

Figueroa KP, Chan P, Schols L, Tanner C, Riess O, Perlman SL, Geschwind DH, Pulst SM (2001) Association of moderate polyglutamine tract expansions in 
the slow calcium-activated potassium channel type 3 with ataxia. Arch Neurol 58: 1649-1653

Fondon JW III, Hammock EA, Hannan AJ, King DG (2008) Simple sequence repeats: genetic modulators of brain function and behavior. Trends Neurosci 31: 328-334

Frei E, Spindler I, Grissmer S, Jager H (2006) Interactions of $\mathrm{N}$-terminal and C-terminal parts of the small conductance $\mathrm{Ca}^{2+}$ activated $\mathrm{K}^{+}$channel, hSK3. Cell Physiol Biochem 18: 165-176

Glatt SJ, Faraone SV, Tsuang MT (2003) CAG-repeat length in exon 1 of KCNN3 does not influence risk for schizophrenia or bipolar disorder: a metaanalysis of association studies. Am J Med Genet B Neuropsychiatr Genet 121B: $14-20$

Grunnet M, Jespersen T, Angelo K, Frokjaer-Jensen C, Klaerke DA, Olesen SP, Jensen BS (2001) Pharmacological modulation of SK3 channels. Neuropharmacology 40: 879-887

Hamill OP, Marty A, Neher E, Sakmann B, Sigworth FJ (1981) Improved patchclamp techniques for high-resolution current recording from cells and cellfree membrane patches. Pfluegers Arch Eur J Physiol 391: 85-100

Hammond RS, Bond CT, Strassmaier T, Ngo-Anh TJ, Adelman JP, Maylie J, Stackman RW (2006) Small-conductance $\mathrm{Ca}^{2+}$-activated $\mathrm{K}^{+}$channel type 2 (SK2) modulates hippocampal learning, memory, and synaptic plasticity. J Neurosci 26: 1844-1853

Huffaker SJ, Chen J, Nicodemus KK, Sambataro F, Yang F, Mattay V, Lipska BK, Hyde TM, Song J, Rujescu D et al (2009) A primate-specific, brain isoform of $\mathrm{KCNH} 2$ affects cortical physiology, cognition, neuronal repolarization and risk of schizophrenia. Nat Med 15: 509-518

Jacobsen JP, Weikop P, Hansen HH, Mikkelsen JD, Redrobe JP, Holst D, Bond CT, Adelman JP, Christophersen P, Mirza NR (2008) SK3 $\mathrm{K}^{+}$channel-deficient mice have enhanced dopamine and serotonin release and altered emotional behaviors. Genes Brain Behav 7: 836-848

Jacobsen JP, Redrobe JP, Hansen HH, Petersen S, Bond CT, Adelman JP, Mikkelsen JD, Mirza NR (2009) Selective cognitive deficits and reduced hippocampal brain-derived neurotrophic factor mRNA expression in smallconductance calcium-activated $\mathrm{K}^{+}$channel deficient mice. Neuroscience 163: $73-81$

Jasinska A, Michlewski G, de Mezer M, Sobczak K, Kozlowski P, Napierala M, Krzyzosiak WJ (2003) Structures of trinucleotide repeats in human transcripts and their functional implications. Nucleic Acids Res 31: 54635468

Ji H, Shepard PD (2006) SK Ca ${ }^{2+}$-activated $\mathrm{K}^{+}$channel ligands alter the firing pattern of dopamine-containing neurons in vivo. Neuroscience 140: 623633

Ji H, Hougaard C, Herrik KF, Strobaek D, Christophersen P, Shepard PD (2009) Tuning the excitability of midbrain dopamine neurons by modulating the $\mathrm{Ca}^{2+}$ sensitivity of SK channels. Eur J Neurosci 29: 1883-1895

Kohler M, Hirschberg B, Bond CT, Kinzie JM, Marrion NV, Maylie J, Adelman JP (1996) Small-conductance, calcium-activated potassium channels from mammalian brain. Science 273: 1709-1714

Koronyo-Hamaoui M, Danziger Y, Frisch A, Stein D, Leor S, Laufer N, Carel C, Fennig S, Minoumi M, Apter A et al (2002) Association between anorexia nervosa and the hsKCa3 gene: a family-based and case control study. Mol Psychiatry 7: 82-85

Koronyo-Hamaoui M, Frisch A, Stein D, Denziger Y, Leor S, Michaelovsky E, Laufer N, Carel C, Fennig S, Mimouni M et al (2007) Dual contribution of NR2B subunit of NMDA receptor and SK3 $\mathrm{Ca}(2+)$-activated $\mathrm{K}^{+}$channel to genetic predisposition to anorexia nervosa. J Psychiatr Res 41: 160-167
Miyake A, Takahashi S, Nakamura Y, Inamura K, Matsumoto S, Mochizuki S, Katou M (2009) Disruption of the ether-a-go-go $\mathrm{K}^{+}$channel gene BEC1/ KCNH3 enhances cognitive function. J Neurosci 29: 14637-14645

Mossner R, Weichselbaum A, Marziniak M, Freitag CM, Lesch KP, Sommer C Meyer J (2005) A highly polymorphic poly-glutamine stretch in the potassium channel KCNN3 in migraine. Headache 45: 132-136

Orr HT, Zoghbi HY (2007) Trinucleotide repeat disorders. Annu Rev Neurosci 30: 575-621

Pedarzani P, Stocker M (2008) Molecular and cellular basis of small-and intermediate-conductance, calcium-activated potassium channel function in the brain. Cell Mol Life Sci 65: 3196-3217

Ribbe K, Friedrichs H, Begemann M, Grube S, Papiol S, Kastner A, Gerchen MF, Ackermann V, Tarami A, Treitz A et al (2010) The cross-sectional GRAS sample: a comprehensive phenotypical data collection of schizophrenic patients. BMC Psychiatry 10: 91

Rimini R, Rimland JM, Terstappen GC (2000) Quantitative expression analysis of the small conductance calcium-activated potassium channels, SK1, SK2 and SK3, in human brain. Brain Res Mol Brain Res 85: 218-220

Ritsner M, Modai I, Ziv H, Amir S, Halperin T, Weizman A, Navon R (2002) An association of CAG repeats at the KCNN3 locus with symptom dimensions of schizophrenia. Biol Psychiatry 51: 788-794

Sailer CA, Kaufmann WA, Marksteiner J, Knaus HG (2004) Comparative immunohistochemical distribution of three small-conductance $\mathrm{Ca}^{2+}$ activated potassium channel subunits, SK1, SK2, and SK3 in mouse brain. Mol Cell Neurosci 26: 458-469

Sander T, Scholz L, Janz D, Epplen JT, Riess O, (1999) Length variation of a polyglutamine array in the gene encoding a small-conductance, calciumactivated potassium channel (hKCa3) and susceptibility to idiopathic generalized epilepsy. Epilepsy Res 33: 227-233

Sarpal D, Koenig JI, Adelman JP, Brady D, Prendeville LC, Shepard PD (2004) Regional distribution of SK3 mRNA-containing neurons in the adult and adolescent rat ventral midbrain and their relationship to dopaminecontaining cells. Synapse 53: 104-113

Sham PC, Curtis D (1995) Monte Carlo tests for associations between disease and alleles at highly polymorphic loci. Ann Hum Genet 59: 97-105

Stackman RW, Hammond RS, Linardatos E, Gerlach A, Maylie J, Adelman JP, Tzounopoulos T (2002) Small conductance $\mathrm{Ca}^{2+}$-activated $\mathrm{K}^{+}$channels modulate synaptic plasticity and memory encoding. J Neurosci 22: 10163 10171

Stober G, Jatzke S, Meyer J, Okladnova O, Knapp M, Beckmann H, Lesch KP (1998) Short CAG repeats within the hSKCa3 gene associated with schizophrenia: results of a family-based study. Neuroreport 9: 3595-3599 Stocker M, Pedarzani P (2000) Differential distribution of three $\mathrm{Ca}(2+)$ activated $\mathrm{K}(+)$ channel subunits, SK1, SK2, and SK3, in the adult rat central nervous system. Mol Cell Neurosci 15: 476-493

Stocker M, Hirzel K, D'Hoedt D, Pedarzani P (2004) Matching molecules to function: neuronal $\mathrm{Ca}^{2+}$-activated $\mathrm{K}^{+}$channels and afterhyperpolarizations. Toxicon 43: 933-949

Vijai J, Kapoor A, Ravishankar HM, Cherian PJ, Kuruttukulam G, Rajendran B, Sridharan R, Rangan G, Girija AS, Jayalakshmi S et al (2005) Protective and susceptibility effects of hSKCa3 allelic variants on juvenile myoclonic epilepsy. J Med Genet 42: 439-442

Wolfart J, Neuhoff H, Franz O, Roeper J (2001) Differential expression of the small-conductance, calcium-activated potassium channel SK3 is critical for pacemaker control in dopaminergic midbrain neurons. J Neurosci 21: $3443-$ 3456

Q1: Author: Please provide the telephone and fax numbers for the second corresponding author. 


\section{EMBO \\ Molecular Medicine}

\author{
Manuscript No.
}

Please correct your galley proofs and return them immediately.

The editors reserve the right to publish your article without your corrections if the proofs do not arrive in time.

Note that the figures in the proofs are produced by laser printer and, therefore, the quality of the halftones is not as high as the final version that will appear in the issue.

Check the enclosed galley proofs very carefully, paying particular attention to the formulae, figures, numerical values, and tabulated data. A black box ( $\mathbf{a})$ or a question between black boxes signals unclear or missing information that specifically requires your attention. Note that the author is liable for damages arising from incorrect statements, including misprints.

\section{(WILEY-VCH}

\section{EMBO Molecular Medicine}

European Molecular Biology Organization

Meyerhofstrasse 1

69117 Heidelberg

Germany

Phone: +49 62218891310

Fax: +4962218891240

E-mail: info@embomolmed.org
Please return your corrected proofs by email or fax within 48 hours.

Fax: +4962218891240 and +496201606202

E-mail: info@embomolmed.org and mblaschke@wiley.com

Please limit corrections to printing errors; costs incurred for any further changes or additions will be charged to the author, unless such changes have been accepted by the editor.

A pdf document including standard correction marks can be found at www.embomolmed.org

Reprints may be ordered by filling out the accompanying form.

\section{EMBO \\ Molecular Medicine}

European Molecular Biology Organization Meyerhofstrasse 1 69117 Heidelberg

Germany 


\section{EMBO Molecular Medicine}

\section{Manuscript No.}

\section{Reprints/lssues}

You will receive a copy of EMBO Molecular Medicine and PDF limited to 25 print-outs free of charge. You also have the opportunity to order reprints, issues or a PDF for an unlimited number of hardcopies at the quoted rates. Whole issues, reprints and high quality PDFs are available at the rates given on the reverse side. After publication the prices of reprints are substantially higher.

For overseas orders please note that you will receive your issues/reprints by airmail. An extra charge will be levied to cover the higher postal rates. If you prefer to receive them by surface mail please sign below.

Please send me and bill me for: reprints entire issues

and send them by

$\square$ surface mail

courier service*

*If you would like to use FedEx please provide your FedEx No:

Mail reprints/ issues to (no P.O. Boxes)

\section{Send bill to}

\section{VAT number}

Tax-free charging can only be processed with the VAT number of the institute/company. To prevent delays with the processing, please provide us with the VAT number with this order.

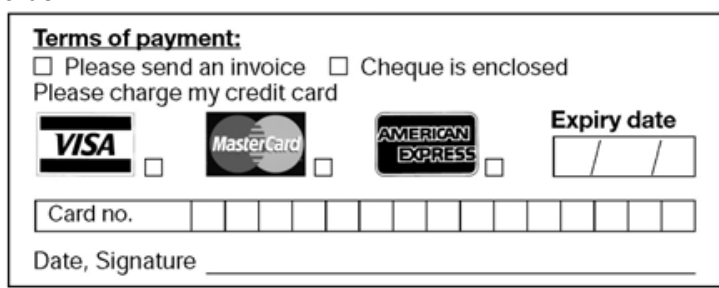

\section{(3) WILEY-VCH}

EMBO Molecular Medicine

European Molecular Biology Organization

Meyerhofstrasse 1

69117 Heidelberg

Germany

Phone: +49 62218891310

Fax: +4962218891240

E-mail: info@embomolmed.org

\section{PDF (unlimited number of hardcopies)}

Please send me a bill for

a PDF file (high resolution)

E-mail address

Please note, that posting of the final published version on the open internet is not permitted.

Mail bill to

Purchase Order No.:

Signature

Date

\section{Subscriptions}

As an author in EMBO Mol Med you obviously appreciate the quality of the journal and value it as a medium for the distribution of your results. We thank you for this support. With the aim of ever increasing the dissemination of information we have maintained low (personal) subscription rates for EMBO Mol Med. Please take advantage of these, ensuring yourself a regular supply of top information and helping us to keep subscription rates down and the accessibility of the journal up. For further information please contact our customer service www.wileycustomerservice.com

\section{Please send me and bill me for}

$\square$ the remaining issues of this volume

$\square$ the complete current volume

$\square$ the next year's volume

$\square$ a library subscription 


\section{Price List for Reprints (2011)}

\section{(WILEY-VCH}

The prices listed below are valid only for orders received in the course of 2011 and before the proofs pass for press. Minimum order is 50 copies. Delivery time will be approximately 3 weeks after the date of publication. If more than 500 copies are ordered, special prices are available upon request. Single issues are available to authors at a reduced price. The prices include mailing and handling charges (with the exception of the additional costs incurred for airmail delivery and courier services). The prices are exclusive of VAT.

Reprints, posters, and issues for overseas orders are shipped by airmail (25.00 Euro surcharge). If you would like to receive them by surface mail please indicate this on the accompanying order form (postage for shipping posters within Europe: 15.00 Euro). If you would like to use a courier service please indicate this on the order form. The cost for the courier service is 75.00 Euro unless you provide your FedEx number, in which case you will be charged the appropriate amount based on your contract with FedEx.

\begin{tabular}{|c|c|c|c|c|c|c|}
\hline \multirow{2}{*}{$\begin{array}{l}\text { Reprints } \\
\text { Size (pages) }\end{array}$} & \multicolumn{6}{|c|}{ Price for orders (in Euro) } \\
\hline & 50 copies & 100 copies & 150 copies & 200 copies & 300 copies & 500 copies \\
\hline $1-4$ & 330.- & 385.- & 425.- & 445.- & 548.- & 752.- \\
\hline $5-8$ & $470-$ & 556.- & 608.- & 636.- & 784.- & 1077.- \\
\hline $9-12$ & 610.- & 717.- & 786.- & 824.- & 1016.- & 1396.- \\
\hline $13-16$ & 744.- & 874.- & 958.- & 1004.- & 1237.- & 1701.- \\
\hline $17-20$ & 885.- & 1040.- & 1138.- & 1196.- & 1489.- & 2022.- \\
\hline $\begin{array}{l}\text { for every additional } \\
4 \text { pages }\end{array}$ & 140.- & 164.- & 175.- & 188.- & 231.- & 315.- \\
\hline Issues & 1 copy: 20 Euro & & PDF (higl & solution) & 330 Euro & \\
\hline
\end{tabular}

\section{Subscription rates for 2011}

The appropriate rate for an institutional subscription is based on the number of Full Time Equivalents (FTE) at the institution purchasing the journal.

\begin{tabular}{|c|c|c|c|}
\hline \multirow[t]{2}{*}{ Costumer Type* } & \multicolumn{3}{|c|}{ Institutional Rates for $Е M B O \mathrm{Mol}$ Med } \\
\hline & small & medium & large \\
\hline FTEAcademic Customer & $\leq 10000$ & $\leq 40000$ & $40000+$ \\
\hline FTE Corporate/Government Customer & $\leq 1000$ & $\leq 10000$ & $10000+$ \\
\hline \multicolumn{4}{|c|}{ Subscription Rate (Print \& electronic/print only or electronic only) } \\
\hline Europe $€$ & $1496 / 1301$ & $1945 / 1691$ & $2528 / 2198$ \\
\hline UKf & $1296 / 1127$ & $1685 / 1465$ & 2190/1904 \\
\hline Switzerland SFr & $2613 / 2272$ & $3397 / 2954$ & $4416 / 3840$ \\
\hline Rest of World US\$ & $1840 / 1600$ & $2392 / 2080$ & $3110 / 2704$ \\
\hline
\end{tabular}

\section{*Rates for hospitals upon request.}

All prices are exclusive of VAT. Postage and handling charges included. Prices are subject to change. Individual rate or member subscriptions may not be resold or used as library copies.

Cancellation of subscriptions: The publisher must be notified not later than three months before the end of the calendaryear.

For further information please contact our customer service www.wileycustomerservice.com

Wiley-VCH Verlag GmbH \& Co. KGaA, Location of the Company: Weinheim; Chairman of the Supervisory Board: Stephen Michael Smith; Trade Register: Mannheim, HRB 432833; General Partner: John Wiley \& Sons GmbH, Location: Weinheim; Trade Register Mannheim, HRB 432296; Managing Directors: Christopher J. Dicks, Bijan Ghawami, William Pesce 
SUPPLEMENTARY MATERIAL

\begin{abstract}
A CAG repeat polymorphism of $K C N N 3$ predicts
SK3 channel function and cognitive performance in schizophrenia
\end{abstract}

\title{
Outline
}

A. Methods

1. Human study

2. Mouse study

3 . In vitro analyses

4. Statistical analyses

B. Supplementary Figures

C. Supplementary Tables

D. Supplementary References 


\section{A. Methods}

\section{Human study}

GRAS: The Göttingen Research Association for Schizophrenia (GRAS) data collection has been approved by the Ethics Committee of the Georg-August-University of Göttingen, Germany (master committee), and the local internal review boards of all participating centers, and complies with the Helsinki declaration. The GRAS project comprises at present 1074 patients diagnosed with DSM-IV-TR schizophrenia (73.2\%; all types, e.g. paranoid, disorganized, catatonic, and undifferentiated, proven or suspected), schizoaffective disorder $(14.8 \%)$ and other psychotic disorders / yet to be confirmed (12.0\%). Patients were included regardless of the stage of the disease (acute, chronic, residual, or remitted). All study participants and, if applicable, their legal representatives gave written informed consent (for more detailed information on the GRAS sample see (Begemann et al, 2010; Ribbe et al, 2010)).

Subjects: A total of $n=1060$ ( $>98.5 \%)$ successfully genotyped GRAS patients were included in the present genetic analysis. Mean age of the patients was (mean \pm s.d.) $39.67 \pm 12.76$ (range 18 to 83$)$ years, with $33.3 \%(n=353)$ being female and $66.7 \%(n=707)$ male. Control subjects were voluntary blood donors, recruited by the Department of Transfusion Medicine at the Georg-August-University of Göttingen according to national guidelines for blood donation. As such, they widely fulfill health criteria, ensured by a broad pre-donation screening process including standardized questionnaires, interviews, hemoglobin, blood pressure, pulse, and body temperature determinations. Of the 1135 (>99\%) successfully genotyped control subjects, $59.0 \%$ were male $(n=670)$ and $41.0 \%$ female $(n=465)$. The average age was $34.57 \pm 12.27$ years, with a range from 18 to 69 years. A large majority of the subjects was of European Caucasian ethnicity, with similar distributions for cases (Caucasian 95.1\%; other ethnicities 1.6\%; unknown 3.3\%) and controls (Caucasian 97.8\%; other ethnicities 2\%; unknown $0.2 \%$ ).

Phenotyping: Comprehensive interviews and testing were carried out by one and the same traveling team of trained examiners (psychiatrists, psychologists) on site at 23 collaborating centers in Germany, using the comprehensive 'GRAS Manual'. Briefly, semi-structured interviews were conducted to explore biographic and family information, level of education, quality of life indicators, disease history or exposure to pre-/peri-/postnatal environmental risk factors. Likewise, the psychopathological profile, psychiatric comorbidities, and current/former treatments were assessed. Psychometric rating, neuropsychological tests and neurological examinations were performed as described in the respective manuals. 


\section{Variables used in the present phenotype analysis:}

Reasoning (LPS3): In the LPS3, 40 rows consisting of 8 symbols each are presented for 5 minutes, and the subject has to identify the symbol that fits least in each row. The number of correctly identified symbols is counted. This task requires reasoning, identification (discrimination and selection) of rules, and resistance against interposed ideas (straight execution) (Horn, 1983).

Executive function (TMT-B): In the trail making test B (TMT-B), subjects are asked to sequentially connect randomly distributed letters and numbers on a page while alternating between the two sets, e.g. 1A2B3C4D etc. This test is a measure for executive function, cognitive flexibility, and sequence alternation (Reitan, 1958; War-Department, 1944).

Word recognition (VLMT): The verbal learning and memory test (VLMT) is a German adaptation of the auditory verbal learning test (AVLT) (Crawford et al, 1989; Rey, 1964). For the (corrected) word recognition performance measure we used, subjects are read a list of words and have to indicate those that had been on a list they learned 30 minutes before in the first part of the test. Distractor items are words from an interference list they had also learned, as well as words that are phonetically or semantically similar to the targets. The corrected recognition performance score is calculated by subtracting the number of wrong answers from the number of correct answers. This test requires verbal learning and memory, as well as a certain degree of cognitive control to discriminate and select between target and distractor items (Helmstaedter et al, 2001).

Divided attention (TAP) and alertness (TAP): 'Testbatterie zur Aufmerksamkeitsprüfung' (TAP) is a computerized German test battery. The subtests divided attention (D3) and alertness (AL) were used. In the divided attention test, subjects are presented in parallel with a visual and an auditory task. They have to press a key as fast as possible whenever crosses presented on a screen build a square, and whenever two similar tones are presented in a row. The number of valid reactions was used in this study. The alertness test is a measure for simple reaction time. In this test, subjects have to react as fast as possible by pressing a key whenever a cross appears on the screen (Zimmermann \& Fimm, 1993).

Tapping and dotting: Tapping and dotting are 'pencil and paper' tests from the MacQuarrie test for mechanical ability, and measure fine motor function, speed, and coordination. In the tapping test, subjects are requested to put into a series of circles 3 pencil dots each as fast as they can. In the dotting test, they are asked to put 1 pencil dot into a series of small circles as accurately and as fast as they can. In both tests, the number of circles completed after 30 seconds is counted (Chapman, 1948; MacQuarrie, 1925, 1953). 
Premorbid Intelligence (MWT-B): In the multiple choice vocabulary test (MWT-B, Lehrl, 1999), subjects are asked without time restrictions to identify 1 correct word in a total of 37 lines, consisting of 5 words ( 1 real word and 4 pronounceable pseudo-words) each,. The MWT-B requires passive word recognition and represents a valid measurement for the premorbid intellectual level. It also correlates well with the global IQ (Lehrl et al, 1995).

Positive and negative syndrome scale (PANSS) for schizophrenia: The PANSS is a rating scale developed to measure symptom severity of schizophrenic patients. It consists of a total of 30 items, divided into the positive symptom scale, negative symptom scale, and general psychopathology scale (Kay et al, 1987). The negative symptom scale of the PANSS was used as covariate to control for the influence of psychopathology on neuropsychological performance. It has been shown that negative symptoms correlate with cognitive performance in schizophrenia, while positive symptoms do not (Dominguez Mde et al, 2009; Nieuwenstein et al, 2001).

Antipsychotic medication dose: Information on regular current medication was obtained directly from chart records or files at the collaborating centers. Antipsychotic medication dose was expressed in chlorpromazine equivalents (Davis, 1976). Chlorpromazine equivalents relate the neuroleptic potency of a compound to that of chlorpromazine in order to obtain comparable units across different medications. Chlorpromazine equivalence factors were taken from the literature or directly obtained from pharmaceutical companies.

\section{Genotyping:}

DNA Extraction and Normalization: Genomic DNA was purified from whole blood using JETQUICK Blood \& Cell Culture DNA Spin Kit (Genomed GmbH, Löhne, Germany) according to the manufacturer's protocol. Resulting DNA samples were aliquoted and stored at $-80^{\circ} \mathrm{C}$. For further analysis, DNA was normalized to $50 \mathrm{ng} / \mu 1$ with an automated robotic platform (Microlab Star, Hamilton, Bonaduz, Switzerland). For quality control, each sample was analyzed with a $0.8 \%$ agarose gel.

Analysis of CAG repeat: The polymorphic CAG repeat in exon1 of $h K C N N 3$ was amplified from genomic DNA by PCR. Primers were chosen according to Austin and colleagues (Austin et al, 1999), resulting in a PCR fragment of $\sim 121 \mathrm{bp}$ : forward 5'-FAM CAG CAG CCC CTG GGA CCC TCG C-3' / reverse 5'-GGA GTT GGG CGA GCT GAG ACA G-3'. For each sample, the reaction mixture $(20 \mu \mathrm{L})$ was prepared in 384 well plates, each containing 50ng of human genomic DNA, $\mathrm{NH}_{4}$ Buffer $(1 \mathrm{x}), 125 \mu \mathrm{M}$ dNTPs, $2.5 \mathrm{mM} \mathrm{MgCl} \mathrm{M}_{2}$, 200nM FAM-labeled forward and reverse primers, and 1U Diamond polymerase (Bioline, 
Luckenwalde, Germany). The cycling program was carried out after a preheating step at $94^{\circ} \mathrm{C}$ for $5 \mathrm{~min}$ and included 30 cycles of (1) denaturation at $94^{\circ} \mathrm{C}$ for $30 \mathrm{~s}$, (2) annealing at $65^{\circ} \mathrm{C}$ for $30 \mathrm{~s}$ and (3) extension at $72^{\circ} \mathrm{C}$ for $60 \mathrm{~s}$ in a DNA Thermal Cycler (PTC-200 MJ Research, BioRad, Munich, Germany). The amplicons were separated using size electrophoresis on the ABI 3730 XL DNA Analyzer (Applied Biosystems, Foster City, USA). Samples were diluted 1:50 with $0.3 \mathrm{mM}$ EDTA and $4 \mu \mathrm{l}$ were mixed with $6 \mu \mathrm{l}$ LIZ-500 Size Standard (Applied Biosystems). Raw data were processed using the Gene Mapper Software 4.0 (Applied Biosystems).

\section{Mouse study}

Animals: All experiments have been approved by the local Animal Care and Use Committee. The establishment of a transgenic mouse line with overexpression of the Kcnn3 gene (here called SK3 T/T) has been reported elsewhere (Bond et al, 2000). SK3 T/T mice have been backcrossed for more then 15 generations with C57BL/6J mice by a commercial breeder (The Jackson Laboratory, Bar Harbor, Maine 04609 USA). We obtained 3 heterozygous SK3 T/T founders as a gift from J.P. Adelman and C.T. Bond (Vollum Institute, OHSU, Portland, OR) and performed another 5 backcrosses with $\mathrm{C} 57 \mathrm{BL} / 6 \mathrm{~J}$ mice before onset of extensive breeding for behavioral experiments. Taken into account that after 20 backcrosses, a SK3 T/T mouse has a widely homogeneous $\mathrm{C} 57 \mathrm{BL} / 6 \mathrm{~J}$ genetic background, we bred mice for experiments by crossing heterozygous females with heterozygous, homozygous and wildtype males in order to obtain a large amount of SK3 T/T and WT males within a short time window. Mice were housed individually in standard plastic cages and maintained in a temperature-controlled environment $\left(21 \pm 2^{\circ} \mathrm{C}\right)$ on a $12 \mathrm{~h}$ light/dark cycle with food and water ad libitum. All experiments have been approved by the local Animal Care and Use Committee.

\section{Mouse behavioral testing:}

Male wildtype and SK3 T/T mice (littermates) were started at the age of 4 months on a battery of behavioral tests, performed in the following order: elevated plus maze, open field, hole board, rota-rod, pre-pulse inhibition, social interaction, sucrose preference, Morris water maze and fear conditioning.

Elevated plus maze: The mouse was placed in the central platform, facing an open arm of the plus-maze. Behavior was recorded by an overhead video camera and a PC equipped with 'Viewer' software (Biobserve, Bonn, Germany) to calculate the time each animal spends in open or closed arms. The proportion of time spent in open arms was used for estimation of open arm aversion (fear equivalent). 
Open field: Spontaneous activity in the open field was tested in a grey Perspex arena $(120 \mathrm{~cm}$ in diameter, $25 \mathrm{~cm}$ high) that was virtually divided into 3 zones: central, intermediate and peripheral. The mouse was placed in the center of the arena and the test started as soon as the mouse reached the wall. Over 7 minutes, the mouse was allowed to freely explore the open field. The behavior was recorded by a PC-linked overhead video camera. 'Viewer' software was used to calculate velocity, distance traveled and time spent in each of the zones. Additionally, the initial latency to reach the wall from the center was recorded.

Hole board: The hole board test measures exploratory activity. The apparatus (TSE, Bad Homburg, Germany) consisted of a $50 \mathrm{~cm} \times 50 \mathrm{~cm} \times 35 \mathrm{~cm}$ transparent perspex chamber with a non-transparent floor raised above the bottom of the chamber. The floor had 16 equally spaced holes, $2.4 \mathrm{~cm}$ in diameter that were fitted with the light barrier sensor $(8 \mathrm{~mm}$ below the floor). Mice were allowed to explore the chamber for $5 \mathrm{~min}$ and the number of explored holes (head dips) was recorded by a PC equipped with the hole board software.

Rota-rod: Rota-rod is a test for motor function, balance and coordination, and comprises a rotating drum (Ugo Basile, Comerio, Varese, Italy) which is accelerated from 4 to 40 revolutions per minute over the course of $5 \mathrm{~min}$. Each mouse was placed individually on a drum and the latency of falling from the drum was recorded using a stop-watch. To assess motor learning, the rota-rod test was repeated $24 \mathrm{~h}$ later.

Pre-pulse inhibition: Pre-pulse inhibition of the startle response was measured in a 4-station testing system (TSE). An experimental session consisted of a $2 \mathrm{~min}$ habituation to the $65 \mathrm{~dB}$ background white noise (continuous throughout the session), followed by a baseline recording for $1 \mathrm{~min}$ at background noise. After baseline recording, 6 pulse alone trials using the startle stimuli of $120 \mathrm{~dB}$ intensity and $40 \mathrm{~ms}$ duration were applied to decrease the influence of within-session habituation (data not included in analysis of PPI). For tests of PPI, the $120 \mathrm{~dB} / 40 \mathrm{~ms}$ startle pulse was applied either alone or preceded by a pre-pulse stimulus of 70 , 75 and $80 \mathrm{~dB}$ intensity and $20 \mathrm{~ms}$ duration. An interval of $100 \mathrm{~ms}$ with background white noise was employed between each pre-pulse and pulse stimulus. All trials were presented in a pseudorandom order with an interval ranging from $8 \mathrm{~s}$ to $22 \mathrm{~s}$. Amplitudes of the startle response were averaged for each individual animal, separately for both types of trials (stimulus alone, stimulus preceded by a pre-pulse). PPI was calculated as a percentage of the startle response using the formula: \% pre-pulse inhibition $=100-[($ startle amplitude to pulse after pre-pulse)/(startle amplitude after pulse only) x 100].

Sociability and social memory test: Sociability and social memory test was performed in rectangular perspex box that was divided with 2 transparent walls, forming 3 chambers (each: 
$20 \mathrm{~cm} \times 40 \mathrm{~cm} \times 22 \mathrm{~cm})$. Dividing walls had rectangular openings $(5 \mathrm{~cm}$ wide) allowing access into each chamber. First, during the habituation phase, the openings were closed and the experimental mouse was placed in the middle chamber and allowed to explore it for $5 \mathrm{~min}$. After the habituation period, the sociability test was performed. For this purpose, an unfamiliar C57BL/6J male mouse (stranger 1) was placed in one of the side chambers. This mouse was enclosed in a small $(7.5 \mathrm{~cm} \times 11.5 \mathrm{~cm} \times 7.5 \mathrm{~cm})$ wire cage, which allowed nose contact between two mice but prevented fighting. An identical empty wire cage was placed in the opposite chamber. Both openings to the side chambers were then opened and the experimental mouse was allowed to freely explore the entire box for $10 \mathrm{~min}$. The time spent in each chamber and the number of entries into each chamber were recorded by the videotracking system 'Viewer'. Subsequently, the social memory test was performed. A second, unfamiliar mouse (stranger 2) was placed into the previously empty wire cage and the experimental mouse had again free access to all chambers for $10 \mathrm{~min}$ and could choose between the first, already explored mouse (stranger 1), and the novel unfamiliar mouse (stranger 2). All mice used as strangers had been previously habituated to the placement in the small wire cage and had no prior contact with the experimental mice.

Sucrose preference test: Mice were first habituated to $1 \%$ sucrose solution which was given in their home cages for $48 \mathrm{~h}$. During the sucrose preference test, mice had free access for $24 \mathrm{~h}$ to two bottles, one with tap water and one with sucrose solution. Consumption of water or sucrose solution was measured by weighing the bottles before and after the session. Sucrose preference was calculated as follows: Preference $=$ [sucrose solution intake $/$ total fluid intake $]^{*} 100$.

Morris water maze: Spatial learning and memory was assessed in a water maze (Morris, 1984). A large circular tank (diameter $1.2 \mathrm{~m}$, depth $0.4 \mathrm{~m}$ ) was filled with opaque water $\left(25 \pm 1^{\circ} \mathrm{C}\right.$, depth $\left.0.3 \mathrm{~m}\right)$ and the escape platform $(10 \mathrm{cmx} 10 \mathrm{~cm})$ was submerged $1 \mathrm{~cm}$ below the surface. The swim patterns were monitored by an overhead video camera and a PC equipped with 'Viewer' software. The escape latency, swim speed, path length, and trajectory of swimming were recorded for each mouse. During the first 2 days, mice were trained to swim to a clearly visible platform (visible platform task) that was marked with a $15 \mathrm{~cm}$ high black flag and placed pseudorandomly in different locations across trials (non-spatial training). The extra-maze cues were hidden during these trials. After 2 days of visible platform training, hidden platform training (spatial training) was performed. For 8 days, mice were trained to find a hidden platform (i.e., the flag was removed) that was located in the center of 1 of the 4 quadrants of the pool. The location of the platform was fixed throughout testing. Mice had to 
navigate using extra-maze cues that were placed on the walls of the testing room. Every day, mice went through four trials with an inter-trial interval of $5 \mathrm{~min}$. The mice were placed into the pool facing the side wall randomly at one of four start locations and allowed to swim until they found the platform, or for a maximum of 90s. Any mouse that failed to find the platform within 90s was guided to the platform. The animal then remained on the platform for $20 \mathrm{~s}$ before being removed from the pool. The day after completion of the hidden platform training, a probe trial was conducted to determine whether mice used a spatial strategy to find the platform. The platform was removed from the pool and the mice were allowed to swim freely for 90s. The percentage of time spent in each quadrant of the pool as well as the number of times the mice crossed the former position of the hidden platform were recorded.

Fear conditioning: Mice were trained within the same session for both contextual and cued fear conditioning. After a 2 min period, during which baseline freezing was assessed, mice received 2 paired presentations of a $10 \mathrm{~s}, 5 \mathrm{kHz}, 85 \mathrm{~dB}$ tone (conditioned stimulus, CS) and a $2 \mathrm{~s}, 0.4 \mathrm{~mA}$ foot shock (unconditioned stimulus, US). The contextual memory was assessed $72 \mathrm{~h}$ after the training. Mice were monitored over $2 \mathrm{~min}$ for freezing in the same conditioning chamber. The cued memory test was performed $4 \mathrm{~h}$ later. For this purpose, mice were placed in a new chamber with novel visual cues and baseline freezing (pre-cue) was measured for $2 \mathrm{~min}$. Thereafter, a $85 \mathrm{~dB}$ tone (conditioned stimulus, CS) was presented for $2 \mathrm{~min}$ and freezing upon tone was quantified. Duration of freezing behavior, defined as the absolute lack of movement (excluding respiratory movements), was recorded by a video camera and a PC equipped with 'Video Freeze' software (MED Associates, St. Albans, Vermont, USA).

\section{Immunohistochemistry:}

Mice were killed by $\mathrm{CO}_{2}$ inhalation and decapitated. Brains were removed and fixed for 3 days in $4 \% \mathrm{w} / \mathrm{v}$ paraformaldehyde in phosphate-buffered saline (PBS), $\mathrm{pH} 7.4$ at $4^{\circ} \mathrm{C}$, followed by paraffin embedding. Brains were serially cut in the sagittal plane at $7 \mu \mathrm{m}$ thickness with a microtome (Model RM2255, Leica, Wetzlar, Germany) and collected on positively charged slides. The plane of sectioning was oriented to match the drawings of the mouse brain atlas (Paxinos \& Franklin, 2001). Following deparaffinizing, tissue sections were washed with Tris-buffered saline (TBS) for $5 \mathrm{~min}$ and incubated with peroxidase block for $15 \mathrm{~min}$ at room temperature. After washing with TBS 3 times, the non-specific binding sites were blocked using $10 \% \mathrm{BSA}$ in TBS for $30 \mathrm{~min}$ at room temperature. Slides were then incubated for $3 \mathrm{~h}$ at room temperature with rabbit polyclonal anti-SK3 antibody (Alomone, Jersualem, Israel; dilution 1:120 v/v), followed by 3 washes for $3 \mathrm{~min}$ each with TBS and 
incubation with peroxidase labeled polymer anti-rabbit antibody (Envision HRP System/DAB for rabbit primary antibodies, DAKO, Glostrup, Denmark) for 30min at room temperature. SK3 staining was visualized with peroxidase and 3,3'-diaminobenzidine chromogen solution. Sections were dehydrated and mounted with xylene-based balsam (Permount, Fisher Scientific, Waltham, USA). The following negative controls were performed: (a) replacement of primary antibody by normal rabbit serum; (b) pre-adsorption of the antibody to the corresponding fusion protein $(1 \mu \mathrm{g}$ peptide per $1 \mu \mathrm{g}$ antibody). Brightfield images were obtained with an Axiovert 200M microscope (Zeiss, Oberkochen, Germany) equipped with a digital camera (AxioCamHR, Zeiss).

\section{Western blot:}

Tissues samples were homogenized in lysis buffer $(75 \mathrm{mM} \mathrm{NaCl}, 25 \mathrm{mM}$ TrisHCl $\mathrm{pH} 7.5$, $0.5 \%$ Triton X-100, 2.5mM EDTA pH 8.0) with complete protease inhibitor cocktail (Roche Diagnostics, Basel, Switzerland), lysed on ice for $20 \mathrm{~min}$, and centrifuged at $4^{\circ} \mathrm{C}$ for $30 \mathrm{~min}$ at $13000 \mathrm{rpm}$. The supernatant was then stored at $-80^{\circ} \mathrm{C}$. Protein concentrations were measured using a Bradford based detergent-compatible colorimetric assay (BioRad). Laemmli sample buffer (Laemmli, 1970) was added to the samples, samples were boiled, and $50 \mu \mathrm{g}$ of total protein per lane was loaded onto SDS-PAGE gels. Proteins were separated by SDS-PAGE on an $8 \%$ polyacrylamide gel before electroblotting onto PVDF Membrane (Bio-Rad) at pH 10. The membrane was blocked with 5\% nonfat milk in TBS $0.1 \%$ Tween-20 (TBST) for $4 \mathrm{~h}$ at $4^{\circ} \mathrm{C}$ before incubation with rabbit polyclonal anti-SK3 antibody (Alomone, 1:2500) in blocking buffer overnight at $4{ }^{\circ} \mathrm{C}$ and before incubation with the mouse monoclonal anti-beta actin antibody (Abcam, Cambridge, UK, 1:10000) in blocking buffer for $1 \mathrm{~h}$ at RT. The membrane was washed 3 times in TBST for 10min, and incubated with the appropriate horseradish-peroxidase-conjugated secondary antibody (sheep anti-mouse IgG: 1:10000 dilution and donkey anti-rabbit IgG: 1:10000 dilution, GE Healthcare, Munich, Germany) in blocking buffer for $1 \mathrm{~h}$ at room temperature. The membrane was washed 2 times in TBST and once in TBS for 10min. Immunoreactivity was visualized by chemoluminescence (ECL, Millipore, Billerica, USA) and quantified by the Quantity One analysis software (Bio-Rad). 


\section{In vitro analysis}

Cloning procedure: The cDNA vector encoding eGFP-labeled SK3 was kindly provided by H.Wulff, UC Davis, CA. The different length of CAG repeats $(11,18$ and 24) were PCR amplified from respective human samples. The following primers were used: hSK3_CAG forward: 5'- CTTCGAATTCCATGGACACTTCTGGGCACTTC -3'; hSK3_CAG reverse: 5'TGTCCCGCCGGTGCACCAGG -3'. The cloning into the vector was performed using EcoRI and SgrAI restriction sites. The resulting constructs eGFPhSK3(CAG) $)_{11}$, eGFPhSK3(CAG) ${ }_{18}$, eGFPhSK3(CAG) $)_{24}$ were verified by sequencing. To analyze a potential influence of the eGFP tag on channel function, we also constructed vectors without tag. Therefore, the eGFP sequence was sequentially cut out with AgeI and BspEI, resulting in the constructs hSK3(CAG) $)_{11}$, hSK3(CAG) ${ }_{18}, \mathrm{hSK} 3(\mathrm{CAG})_{24}$.

Transfection: For electrophysiological experiments, cells were transfected using Lipofectamine 2000 (Invitrogen, Karlsruhe, Germany) following the manufacturer's guidelines. Stable cell pools were obtained by selection with $300 \mu \mathrm{g} / \mathrm{mL}$ G-418 (Invitrogen). There was no detectable difference in terms of electrophysiological characteristics between transiently (detected through eGFP fluorescence) and stably transfected cells. Transfected cells were grown for $24-72 \mathrm{~h}$ on fibronectin-coated glass cover slips. Representative fluorescence images of living cells were taken under an epifluorescence microscope; nuclei were stained with Hoechst33342 (Invitrogen). hSK3(CAG) 11, hSK3(CAG) 18 , and hSK3(CAG) $)_{24}$ were measured in transiently transfected cells only.

Electrophysiology: All measurements were conducted by a blinded investigator. Electrophysiological experiments were performed at room temperature. Macroscopic currents were recorded in the whole-cell configuration of the patch-clamp technique (Hamill et al, 1981) using an EPC-9 amplifier (HEKA, Lambrecht, Germany). Patch pipettes with a tip resistance of $1.5-2 \mathrm{M} \Omega$ were made from Corning \#0010 capillary glass (WPI, Berlin, Germany). Series resistance was compensated by $60-80 \%$. Both series resistance and total cell capacity were determined by automatic cancellation of capacity transients. The internal solution contained (in $\mathrm{mM}$ ) $160 \mathrm{KCl}, 0.5 \mathrm{MgCl}_{2}, 10 \mathrm{EGTA}, 9.5 \mathrm{CaCl}_{2}$, (free $\mathrm{Ca}^{2+} 1.03 \mu \mathrm{M}$ ), $10 \mathrm{Hepes} / \mathrm{KOH} \mathrm{pH} 7.35$. The control external recording solution contained (in $\mathrm{mM}$ ) $160 \mathrm{NaCl}$, $2.5 \mathrm{KCl}, 2 \mathrm{CaCl}_{2}, 1 \mathrm{MgCl}_{2}, 8$ glucose, $10 \mathrm{HEPES} / \mathrm{NaOH}, \mathrm{pH}$ 7.4. Apamin (100nM, Alomone) was added to the control external solution from a $50 \mu \mathrm{M}$ stock, and cells were superfused with the apamin-containing solution using a focal perfusion system (ALA scientific instruments, Farmingdale, NY). Currents elicited by a $500 \mathrm{~ms}$ voltage ramp from -80 to $+80 \mathrm{mV}$ were digitized at $4 \mathrm{kHz}$. Apamin-sensitive currents were determined by off-line subtraction of traces 
obtained in the presence of apamin from control traces, using FitMaster (HEKA) and IgorPro (WaveMetrics, Lake Oswego, OR). Data shown in text and legends represent mean \pm s.e.m. for the indicated number of experiments. To fit the data, we used a linear current/voltage function with a positive block with the form:

$$
f(V)=G\left(V-V_{E}\right) \frac{1}{1+e^{\frac{-\left(V-V_{b}\right)}{k}}}
$$

where $\mathrm{G}$ is the overall conductance, $\mathrm{V}_{\mathrm{E}}$ is the equilibrium potential $(-107 \mathrm{mV}$ under our experimental conditions for a $\mathrm{K}^{+}$-selective channel), $\mathrm{V}_{\mathrm{b}}$ is the voltage at which half-maximal block occurs and $k$ is the slope of the blocking function.

Western blot confirmation of glutamine repeat lengths: Experiments were performed using rabbit polyclonal anti-SK3 (Alomone, 1:2500) and mouse monoclonal anti- $\alpha$-tubulin antibodies (SIGMA-Aldrich, Taufkirchen, Germany, 1:20000) and appropriate horseradishperoxidase-conjugated secondary antibodies (anti-rabbit IgG: 1:5000 and anti-mouse IgG: 1:10000, SIGMA-Aldrich). Immunoreactivity was visualized by chemoluminescence (ECL, Millipore).

\section{Statistical analyses}

Case-control study: The sum of allelic repeat lengths of all individuals was analyzed. To account for the degree of heterogeneity between the 2 alleles, the difference between the allelic repeat lengths was also calculated. $p$ values for the distribution of genotypes among schizophrenic and control samples were determined by Monte Carlo tests with Clump software (Sham \& Curtis, 1995). (http://www.mds.qmw.ac.uk/statgen/dcurtis/software.html). This program derives a $\chi^{2}$ value from a $2 \mathrm{xm}$ table by 'clumping' columns together into a new $2 \times 2$ table in a way designed to maximize the $\chi^{2}$ value. The unbiased significance is assessed by a Monte Carlo approach generating $2 \mathrm{xm}$ tables with the same row and column totals as the original table, clumping them into $2 \times 2$ tables to produce maximal $\chi^{2}$ values, and counting the number of times the $\chi^{2}$ value of the clumped real table is achieved or exceeded by the randomly simulated data.

PGAS: Statistical analyses were performed using R2.10.0 (http://cran.r-project.org). All cognitive phenotypes were represented such that larger values correspond to better performance: For this purpose, a sign change was applied to all excecution times. After selection of the relevant subsample (see below), metric target and control phenotypic variables were standardized by Blom transformation (Blom, 1958) prior to statistical analysis. 
The Blom transformation is a probit transformation of the ranks $r_{i}$ obtained on all $n$ available (non-missing) values of a specific phenotype, $y_{i}=\Phi^{-1}\left(\left(r_{i}-3 / 8\right) /(n+1 / 4)\right)$ where $\Phi^{-1}$ is the quantile function of the standard normal distribution. The resulting standardized phenotypes are normally distributed with zero mean and variance one. They were analyzed by linear models, adjusting for covariates sex and age, additionally for medication (all cognitive phenotypes, PANSS-positive and negative control variables) and for negative symptoms (all cognitive phenotypes). The influence of allelic repeat length sum was studied by a regression model (main table 1) and by a classification model (Table S2). The regression model estimates the change of the covariate-adjusted mean phenotype value per additional repeat in the sum of repeat lengths of the two alleles. We excluded rare extreme observations of allelic repeat length sum from the regression analysis and non-native German speakers with language problems.

The classification model estimates and tests the difference in the covariate-adjusted phenotype means between groups with low (below-median) and high (above-median) allelic repeat length sum. The sample median of allelic repeat length sum was 35 . Individuals with this value were excluded from the classification analysis as well as non-native German speakers with language problems. Multiple testing adjusted significant thresholds were determined by Bonferroni-adjustment or by permutation test (to account for correlations between cognitive phenotypes). The permutation test simulated the distribution of wrong test decisions under the null-hypothesis of no genotype-phenotype association by generating replications of the original sample, each with permuted assignment of the individual genotype. This conserves the underlying correlations between cognitive traits and the underlying correlations between traits and covariates. The multiple-testing adjusted significance threshold was determined such that the probability of at least one wrong test decision was $\leq 5 \%$ for the considered set of tests on $n=50000$ replications of the original sample with permuted genotype-phenotype assignment. 


\section{B. Supplementary Figures}

\section{A Anhedonia test}

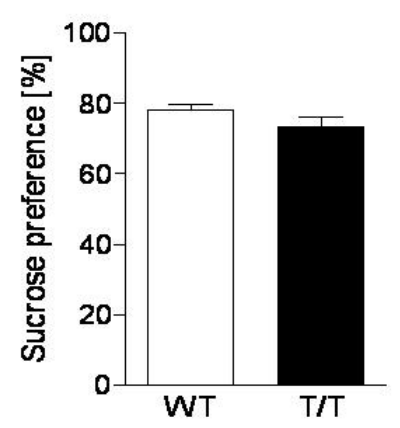

B Sociability

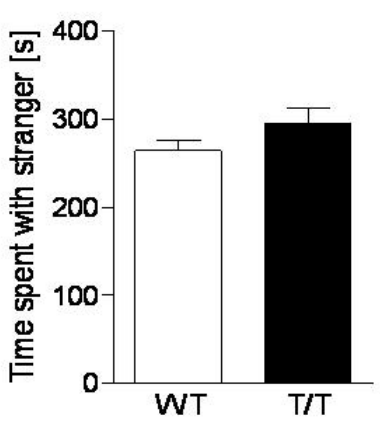

C Social memory

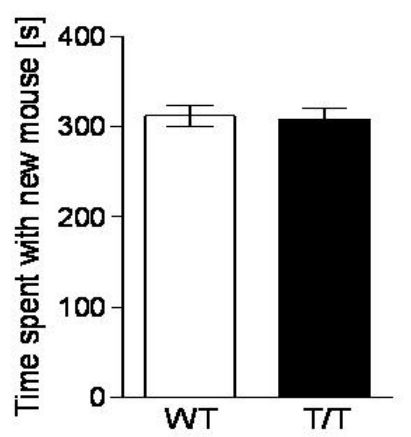

D Pre-pulse inhibition

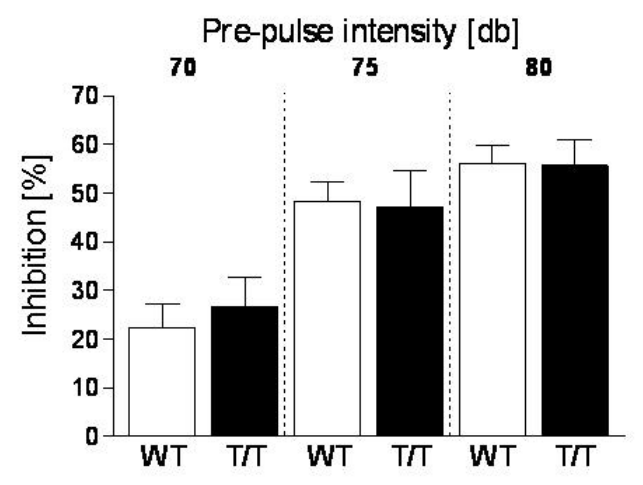

E MWM: probe trial

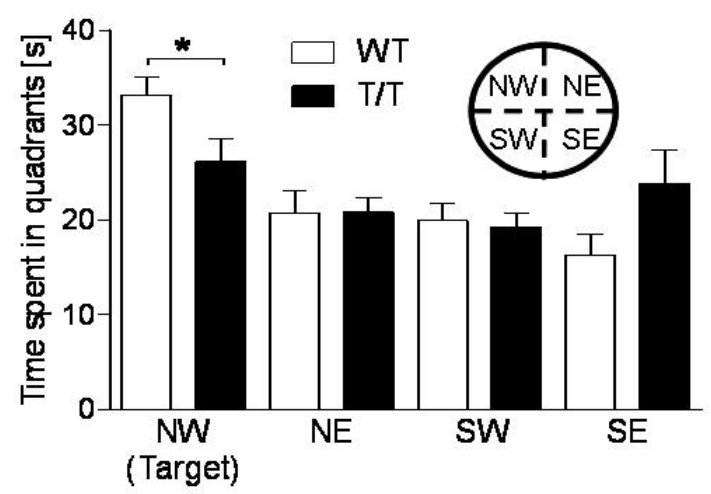

Figure S1: Additional behavioral results in SK3 T/T versus WT mice

(A-D) The 2 genotypes did not differ in sucrose preference (A), sociability (B), social memory (C), and pre-pulse inhibition of the startle response at 3 different pre-pulse intensities (D). (E) The probe trial of the Morris water maze test revealed genotype differences (2-way repeated measures ANOVA, quadrant $\mathrm{x}$ genotype interaction: $\left.\mathrm{F}_{3,78}=2.70, p=0.05\right) . \mathrm{T} / \mathrm{T}$ mice spent less time exploring the target quadrant ( ${ }^{*}$ Student's $t$-test, $t_{1,26}=2.25, p=0.033$ ). For all experiments: $n=13-15$; data presented as mean \pm s.e.m.; $*$ indicates $p<0.05$. 
A

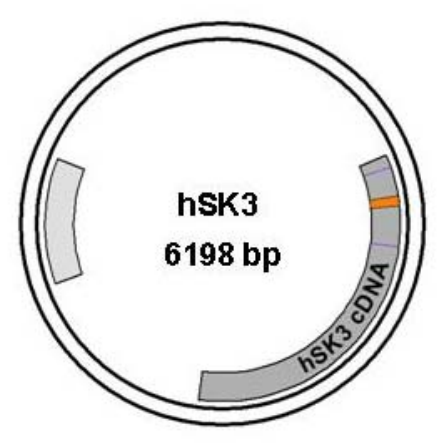

B

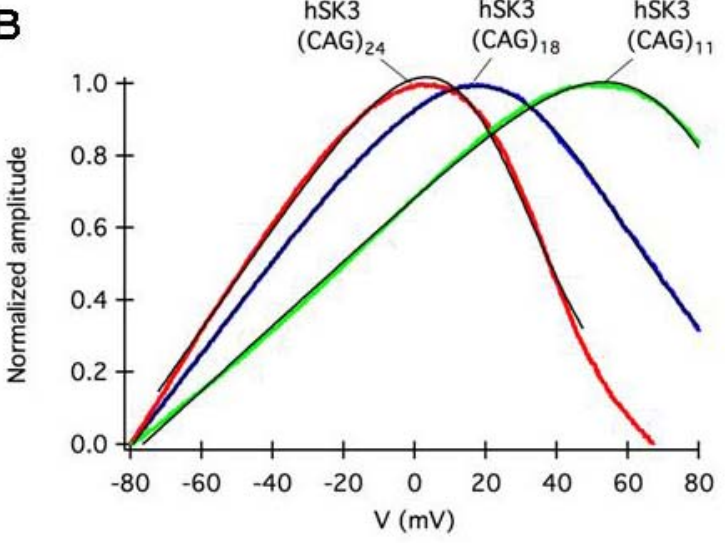

Figure S2: KCNN3 CAG repeat length affects electrophysiological properties of SK3 also in the absence of the eGFP tag

(A) The eGFP in all constructs was removed, resulting in hSK3(CAG) ${ }_{n}$. Indicated are size, locations of the CAG repeat (labeled in orange) and of the primers (labeled in purple). (B) Average apaminsensitive current amplitude (normalized versus the corresponding current maximum) for clones $\mathrm{hSK} 3(\mathrm{CAG})_{11}$ (green), hSK3(CAG) ${ }_{18}$ (blue) and hSK3(CAG) $)_{24}$ (red). The black line represents the fit to an I/V function with voltage dependent block. Voltage for half-maximal block was $86.1 \pm 0.08 \mathrm{mV}$, $44.4 \pm 0.02 \mathrm{mV}(n=7)$ and $25.9 \pm 0.07 \mathrm{mV}(n=7)$ for hSK3(CAG) $)_{11}, \mathrm{hSK} 3(\mathrm{CAG})_{18}$ and hSK3(CAG) $)_{24}$ respectively. 


\section{Supplementary Tables}

Table S1:

Allelic repeat lengths differences and sums - Observed combinations and their counts

\begin{tabular}{|c|c|c|c|c|c|c|c|c|c|c|}
\hline & \multicolumn{7}{|c|}{ Difference } \\
\hline Sum & $\mathbf{0}$ & $\mathbf{1}$ & $\mathbf{2}$ & $\mathbf{3}$ & $\mathbf{4}$ & $\mathbf{5}$ & $\mathbf{6}$ & $\mathbf{7}$ & $\mathbf{8}$ & $\mathbf{9}$ \\
\hline $\mathbf{2 4}$ & 5 & & 1 & & & & & & & \\
\hline $\mathbf{2 5}$ & & 2 & & & & & & & & \\
\hline $\mathbf{2 6}$ & 1 & & 1 & & & & 2 & & & \\
\hline $\mathbf{2 7}$ & & & & 3 & & & & 2 & & \\
\hline $\mathbf{2 8}$ & 1 & & 3 & & 9 & & & & 3 & \\
\hline $\mathbf{2 9}$ & & & & 4 & & 23 & & & & 1 \\
\hline $\mathbf{3 0}$ & 4 & & 5 & & 14 & & 15 & & & \\
\hline $\mathbf{3 1}$ & & 8 & & 6 & & 18 & & 9 & & \\
\hline $\mathbf{3 2}$ & 13 & & 23 & & 7 & & 7 & & 6 & \\
\hline $\mathbf{3 3}$ & & 57 & & 22 & & 2 & & 3 & & \\
\hline $\mathbf{3 4}$ & 52 & & 82 & & 9 & & 2 & & & \\
\hline $\mathbf{3 5}$ & & 151 & & 20 & & 6 & & & & \\
\hline $\mathbf{3 6}$ & 121 & & 49 & & 18 & & & & & \\
\hline $\mathbf{3 7}$ & & 66 & & 20 & & 1 & & & & \\
\hline $\mathbf{3 8}$ & 12 & & 40 & & 4 & & 2 & & & \\
\hline $\mathbf{3 9}$ & & 13 & & 7 & & 1 & & & \\
\hline $\mathbf{4 0}$ & 4 & & 5 & & 4 & & & & \\
\hline $\mathbf{4 1}$ & & 1 & & & 2 & & & \\
\hline $\mathbf{4 2}$ & & & 1 & & & & & \\
\hline
\end{tabular}

Shown are the counts of all observed individual combinations for the difference and sum of allelic repeat lengths of the 2 alleles in schizophrenic patients without language problems $(n=973)$. Empty cells are given in case of no observations. Regression analysis was applied to the shaded region of sum values (ranging from 28 to 40), omitting rare observations with extreme sum values. The reason for this exclusion is that the power of the regression analysis is optimal when the analysis is focused on the range of sum values where the transition between good and poor functioning of the SK3 channel occurs. Extreme sum values were rare and not expected to be part of this transition region. For classification analysis, the median sum value of 35 (darker shade) was omitted, contrasting the 2 groups with low (below-median) versus high (above-median) sum values and including rare extremes. The reason behind the exclusion of the median sum value is that the power of the classification analysis is optimal when only groups with good and poor channel function are compared, omitting sum values of the transition region. 
Table S2:

Classification model: Association of low versus high SK3 allelic repeat lengths sum with target and control phenotypes

\begin{tabular}{|c|c|c|c|c|}
\hline \multirow{2}{*}{ Phenotypes } & \multicolumn{2}{|c|}{ Difference in class means } & \multicolumn{2}{|c|}{ Statistical test } \\
\hline & estimate & 95\% confidence interval & t-value (1df) & $p$ value \\
\hline \multicolumn{5}{|l|}{ Cognitive target variables: } \\
\hline Combined (multivariate) & 0.1544 & $0.0654,0.2434$ & 3.4004 & $0.0007^{\mathrm{a}}$ \\
\hline \multicolumn{5}{|l|}{ Individual (univariate) } \\
\hline Reasoning & 0.1500 & $0.0322,0.2679$ & 2.4989 & $0.0127^{b}$ \\
\hline Executive function & 0.1647 & $0.0455,0.2838$ & 2.7141 & $0.0068^{\mathrm{b}}$ \\
\hline Word recognition & 0.1449 & $0.0185,0.2713$ & 2.2508 & $0.0247^{b}$ \\
\hline Divided attention & 0.2015 & $0.0647,0.3382$ & 2.8918 & $0.0040^{b}$ \\
\hline \multicolumn{5}{|l|}{ Cognitive control variables: } \\
\hline Combined (multivariate) & 0.0527 & $-0.0410,0.1465$ & 1.1025 & $0.2703^{a}$ \\
\hline \multicolumn{5}{|l|}{ Individual (univariate) } \\
\hline Dotting & 0.0527 & $-0.0676,0.1731$ & 0.8605 & 0.3898 \\
\hline Tapping & 0.0609 & $-0.0594,0.1812$ & 0.9944 & 0.3203 \\
\hline Alertness & 0.0564 & $-0.0641,0.1769$ & 0.9187 & 0.3586 \\
\hline Premorbid Intelligence & 0.0550 & $-0.0763,0.1864$ & 0.8224 & $0.4111^{\mathrm{a}}$ \\
\hline \multicolumn{5}{|c|}{ Disease-related control variables: } \\
\hline PANSS positive symptoms & 0.0357 & $-0.0934,0.1648$ & 0.5429 & $0.5874^{\mathrm{a}}$ \\
\hline PANSS negative symptoms & 0.0226 & $-0.1144,0.1596$ & 0.3236 & $0.7463^{\mathrm{a}}$ \\
\hline \multicolumn{5}{|c|}{ Disease-unrelated control variable: } \\
\hline Body length & 0.0755 & $-0.0297,0.1806$ & 1.4088 & $0.1593^{\mathrm{a}}$ \\
\hline
\end{tabular}

${ }^{a, b}$ Multiple testing corrected significance thresholds: ${ }^{a}$ first level of tests $p \leq 0.0100$ (Bonferroni), ${ }^{b}$ second level of tests $p \leq 0.0141$ (permutation test with 50000 permutations to account for correlations between phenotypes).

Association analyses of low (below-median; <35) versus high (above-median; >35) allelic repeat lengths sum with mean value of target and control phenotypes. Omitted were individuals with sample median repeat lengths sum $35(n=177)$ and non-native German speakers with language problems ( $n=87$ ), to give a total of $n=796$ to be analyzed. Phenotypes were adjusted for sex and age, for medication (all cognitive phenotypes, PANSS-positive, PANSS-negative) and for negative symptoms (all cognitive phenotypes). All phenotypes were standardized to zero mean and variance one: larger values for cognitive phenotypes correspond to better performance. Like Cohen's d, the genetically induced effect size (class mean difference) is quantified relative to the standard deviation of the trait. 
Table S3a:

Raw data of cognitive target and control variables in low* $(n=425)$ and high* $(n=371)$ allelic repeat lengths sum carriers as well as in the total GRAS sample ( $n=1060)$ and in healthy controls (normative samples)

\begin{tabular}{|c|c|c|c|c|c|c|c|c|c|}
\hline & \multicolumn{2}{|c|}{ Low allelic repeat lengths sum* } & \multicolumn{2}{|c|}{ High allelic repeat lengths sum } & \multicolumn{2}{|c|}{ total GRAS sample } & \multicolumn{3}{|c|}{$\begin{array}{l}\text { normative data (PR)' or mean values of healthy } \\
\text { controls }\end{array}$} \\
\hline & mean $\pm \mathbf{s d}$ & range & mean \pm sd & range & mean \pm sd & range & $n$ & $\begin{array}{c}\mathbf{P R}^{1} \\
\text { (Percentile Rank) }\end{array}$ & mean $\pm s d$ \\
\hline \multicolumn{10}{|c|}{ Cognitive target variables (individual) } \\
\hline Reasoning (LPS) & $20.04 \pm 6.76$ & $2-37$ & $20.76 \pm 6.56$ & $5-38$ & $20.35 \pm 6.75$ & $2-38$ & $1556^{a}$ & 31 & - \\
\hline Executive function (TMT-B) ${ }^{\circ}$ & $139.02 \pm 116.05$ & $27-1118$ & $133.86 \pm 108.70$ & $32-836$ & $136.96 \pm 112.49$ & $27-1118$ & $24^{b}$ & $<10$ & $71.50 \pm 31.07$ \\
\hline Word recognition (VLMT) & $9.79 \pm 5.60$ & $-16-15$ & $10.20 \pm 5.52$ & $-23-15$ & $9.93 \pm 5.54$ & $-23-15$ & $515^{c}$ & 10 & \\
\hline Divided attention (TAP) & $25.37 \pm 5.13$ & $7-33$ & $26.21 \pm 5.17$ & $6-33$ & $25.69 \pm 5.20$ & $6-33$ & - & - & - \\
\hline \multicolumn{10}{|c|}{ Cognitive control variables (individual) } \\
\hline Dotting & $45.71 \pm 13.55$ & $13-100$ & $45.80 \pm 13.60$ & $8-85$ & $45.59 \pm 13.70$ & $8-100$ & $103^{d}$ & - & $63.24 \pm 11.03$ \\
\hline Tapping & $28.45 \pm 8.41$ & $8-65$ & $28.70 \pm 8.82$ & $4-59$ & $28.36 \pm 8.67$ & $4-65$ & $103^{d}$ & - & $37.63 \pm 7.04$ \\
\hline Alertness (TAP), reaction time ${ }^{\circ}$ & $333.61 \pm 135.74$ & $178.00-1257.00$ & $332.77 \pm 147.43$ & $166.50-1355.00$ & $337.62 \pm 146.05$ & $166.50-1355.00$ & $120^{e}$ & 3 & - \\
\hline Premorbid IQ (MWT-B) & $25.70 \pm 6.37$ & $4-37$ & $25.90 \pm 6.19$ & $5-37$ & $25.79 \pm 6.35$ & $4-37$ & $1952^{f}$ & 39.2 & - \\
\hline
\end{tabular}

* Non-native German speakers with language problems $(n=87$ ) were excluded. Low (below-median; $<35$ ) and high (above median; $>35$ ) allelic repeat lengths sum carriers presented; individuals with an allelic repeat lengths sum of 35 ( $n=177$ ) were excluded. 1 Higher scores reflect better performance, except for TMT-B and Alertness
1 Percentile ranks $(\mathrm{PR})<15$ indicate that the mean or the median of the total sample is below average in comparison to a normative sample

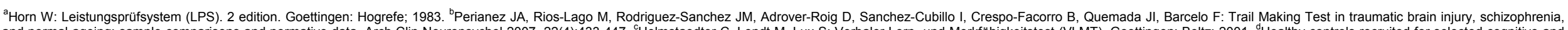

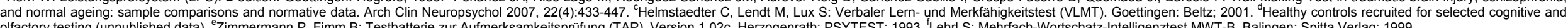


Table S3b:

Raw data of cognitive target and control variables in carriers of different allelic repeat lengths sums. Non-native German speakers with language problems $(n=87)$ were excluded.

\begin{tabular}{|c|c|c|c|c|c|c|c|c|c|}
\hline Allelic repeat lengths sum & $28+29+30$ & 31 & 32 & 33 & 34 & 35 & 36 & 37 & $38+39+40$ \\
\hline$n$ (range) & 82 & 41 & 56 & 84 & 145 & 177 & 188 & 87 & 92 \\
\hline \multicolumn{10}{|c|}{ Cognitive target variables (individual) } \\
\hline Reasoning (LPS) & $20.16 \pm 6.07$ & $20.76 \pm 6.48$ & $18.69 \pm 6.76$ & $19.69 \pm 6.90$ & $20.17 \pm 7.24$ & $20.24 \pm 7.10$ & $20.98 \pm 6.39$ & $20.60 \pm 6.43$ & $20.37 \pm 7.11$ \\
\hline Executive function (TMT-B) ${ }^{\circ}$ & $159.98 \pm 142.94$ & $146.68 \pm 115.65$ & $130.76 \pm 100.67$ & $134.90 \pm 85.67$ & $132.36 \pm 120.30$ & $138.73 \pm 112.44$ & $133.53 \pm 115.13$ & $124.99 \pm 90.83$ & $142.83 \pm 111.73$ \\
\hline Word recognition (VLMT) & $9.24 \pm 5.65$ & $9.24 \pm 6.56$ & $10.22 \pm 5.85$ & $8.89 \pm 6.03$ & $10.37 \pm 4.89$ & $9.70 \pm 5.44$ & $9.94 \pm 6.14$ & $10.14 \pm 4.90$ & $10.74 \pm 4.69$ \\
\hline Divided attention (TAP) & $24.77 \pm 5.65$ & $25.29 \pm 4.59$ & $26.22 \pm 4.43$ & $25.25 \pm 5.47$ & $25.38 \pm 5.10$ & $25.38 \pm 5.38$ & $26.44 \pm 5.10$ & $26.33 \pm 4.92$ & $25.71 \pm 5.37$ \\
\hline \multicolumn{10}{|c|}{ Cognitive control variables (individual) } \\
\hline Dotting & $44.40 \pm 12.85$ & $44.83 \pm 12.23$ & $47.34 \pm 14.53$ & $46.91 \pm 14.00$ & $44.93 \pm 13.37$ & $44.86 \pm 14.28$ & $46.38 \pm 13.09$ & $45.23 \pm 12.63$ & $45.45 \pm 15.41$ \\
\hline Tapping & $28.37 \pm 8.50$ & $28.98 \pm 8.58$ & $28.87 \pm 8.74$ & $28.13 \pm 8.24$ & $28.14 \pm 8.32$ & $27.44 \pm 8.94$ & $28.79 \pm 8.76$ & $28.71 \pm 7.70$ & $28.90 \pm 10.24$ \\
\hline Alertness (TAP), reaction time ${ }^{\circ}$ & $358.87 \pm 179.15$ & $309.65 \pm 109.98$ & $316.06 \pm 101.69$ & $337.25 \pm 133.57$ & $331.03 \pm 127.06$ & $357.81 \pm 165.44$ & $330.35 \pm 149.30$ & $337.29 \pm 155.97$ & $335.88 \pm 138.40$ \\
\hline Premorbid IQ (MWT-B) & $25.50 \pm 6.61$ & $24.68 \pm 6.08$ & $25.23 \pm 5.88$ & $25.61 \pm 6.85$ & $26.28 \pm 6.01$ & $25.78 \pm 6.66$ & $26.62 \pm 5.86$ & $25.64 \pm 6.07$ & $24.52 \pm 6.88$ \\
\hline
\end{tabular}

- Higher scores reflect better performance, except for TMT-B and Alertness 


\section{Supplementary References}

Austin CP, Holder DJ, Ma L, Mixson LA, Caskey CT (1999) Mapping of hKCa3 to chromosome 1q21 and investigation of linkage of CAG repeat polymorphism to schizophrenia. Mol Psychiatry 4: 261-266

Begemann M, Grube S, Papiol S, Malzahn D, Krampe H, Ribbe K, Friedrichs H, Radyushkin KA, El-Kordi A, Benseler F, Hannke K, Sperling S, Schwerdtfeger D, Thanhauser I, Gerchen MF, Ghorbani M, Gutwinski S, Hilmes C, Leppert R, Ronnenberg A, Sowislo J, Stawicki S, Stodtke M, Szuszies C, Reim K, Riggert J, Eckstein F, Falkai P, Bickeboller H, Nave KA, Brose N, Ehrenreich H (2010) Modification of cognitive performance in schizophrenia by complexin 2 gene polymorphisms. Arch Gen Psychiatry 67: 879-888

Blom G (1958) Statistical Estimates and Transformed Beta Variables, New York: John Wiley and Sons, Inc.

Bond CT, Sprengel R, Bissonnette JM, Kaufmann WA, Pribnow D, Neelands T, Storck T, Baetscher M, Jerecic J, Maylie J, Knaus HG, Seeburg PH, Adelman JP (2000) Respiration and parturition affected by conditional overexpression of the $\mathrm{Ca} 2+$-activated $\mathrm{K}+$ channel subunit, SK3. Science 289: 1942-1946

Chapman RL (1948) The MacQuarrie test for mechanical ability. Psychometrika 13: 175-179

Crawford JR, Stewart LE, Moore JW (1989) Demonstration of Savings on the AVLT and Development of a Parallel Form. Journal of Clinical and Experimental Neuropsychology 11: 975-981

Davis JM (1976) Comparative doses and costs of antipsychotic medication. Arch Gen Psychiatry 33: 858-861

Dominguez Mde G, Viechtbauer W, Simons CJ, van Os J, Krabbendam L (2009) Are psychotic psychopathology and neurocognition orthogonal? A systematic review of their associations. Psychol Bull 135: 157-171

Hamill OP, Marty A, Neher E, Sakmann B, Sigworth FJ (1981) Improved patch-clamp techniques for high-resolution current recording from cells and cell-free membrane patches. Pfluegers Arch Eur J Physiol 391: 85-100

Helmstaedter C, Lendt M, Lux S (2001) Verbaler Lern- und Merkfähigkeitstest (VLMT), Goettingen: Beltz.

Horn W (1983) Leistungsprüfsystem (LPS), 2 edn. Goettingen: Hogrefe.

Kay SR, Fiszbein A, Opler LA (1987) The positive and negative syndrome scale (PANSS) for schizophrenia. Schizophr Bull 13: 261-276

Laemmli UK (1970) Cleavage of structural proteins during the assembly of the head of bacteriophage T4. Nature 227: 680-685

Lehrl S (1999) Mehrfach-Wortschatz-Intelligenztest MWT-B, Balingen: Spitta Verlag. 
Lehrl S, Triebig G, Fischer B (1995) Multiple choice vocabulary test MWT as a valid and short test to estimate premorbid intelligence. Acta Neurol Scand 91: 335-345

MacQuarrie TW (1925, 1953) MacQuarrie Test for Mechanical Ability., Monterey: CA: California Test Bureau/McGraw-Hill.

Morris R (1984) Developments of a water-maze procedure for studying spatial learning in the rat. Journal of neuroscience methods 11: 47-60

Nieuwenstein MR, Aleman A, de Haan EHF (2001) Relationship between symptom dimensions and neurocognitive functioning in schizophrenia: a meta-analysis of WCST and CPT studies. Journal of Psychiatric Research 35: 119-125

Paxinos G, Franklin KBJ (2001) The mouse brain in stereotaxic coordinates, 2nd edn. San Diego: Academic Press.

Perianez JA, Rios-Lago M, Rodriguez-Sanchez JM, Adrover-Roig D, Sanchez-Cubillo I, Crespo-Facorro B, Quemada JI, Barcelo F (2007) Trail Making Test in traumatic brain injury, schizophrenia, and normal ageing: sample comparisons and normative data. Arch Clin Neuropsychol 22: 433-447

Reitan RM (1958) The validity of the Trail Making Test as an indicator of organic brain damage. Perceptual and Motor Skills 8: 271-276

Rey A (1964) L'examen clinique en psychologie, Paris: Presses Universitaires de France.

Ribbe K, Friedrichs H, Begemann M, Grube S, Papiol S, Kastner A, Gerchen MF, Ackermann V, Tarami A, Treitz A, Flogel M, Adler L, Aldenhoff JB, Becker-Emner M, Becker T, Czernik A, Dose M, Folkerts H, Freese R, Gunther R, Herpertz S, Hesse D, Kruse G, Kunze H, Franz M, Lohrer F, Maier W, Mielke A, Muller-Isberner R, Oestereich C, Pajonk FG, Pollmacher T, Schneider U, Schwarz HJ, Kroner-Herwig B, Havemann-Reinecke U, Frahm J, Stuhmer W, Falkai P, Brose N, Nave KA, Ehrenreich H (2010) The cross-sectional GRAS sample: a comprehensive phenotypical data collection of schizophrenic patients. BMC Psychiatry 10: 91

Sham PC, Curtis D (1995) Monte Carlo tests for associations between disease and alleles at highly polymorphic loci. Ann Hum Genet 59: 97-105

War-Department (1944) Army Individual Test Battery. Manual of Directions and Scoring, Washington, D.C.: War Department, Adjutant General's Office.

Zimmermann P, Fimm B (1993) Testbatterie zur Aufmerksamkeitsprüfung (TAP). Version 1.02c, Herzogenrath: PSYTEST. 


\section{SUMMARY AND CONCLUSIONS}

Genetic association, linkage and family studies in the past decades show that there is a strong genetic component to schizophrenia. However, even the recent GWAS and CNV studies, analyzing huge patient cohorts, have not been able to detect a disease gene confirming that schizophrenia is polygenic and that each patient/family probably does have a different "risk" constellation of genotypes leading to the same diagnosis. The translation of genetic findings to biological mechanisms is still in its infancy.

With our GRAS cohort, we have the unique opportunity to analyze how common variations in genes of interest, e.g. suggested by the GWAS, contribute to the phenotype. With the first two studies of this kind we were able to prove that our database is a valuable tool and that the sample size is adequate for this kind of analysis.

Analyzing SK3 and CPLX2 showed that common variations (microsatellites and single nucleotide polymorphisms) contribute to specific cognitive domains in our schizophrenia sample. Interestingly, we could show for both genes that there are similar effects in mice and that an altered biological function is -at least in part- responsible for our findings. The CAG repeat in SK3 undoubtedly influences the electrophysiological properties of the resulting channel and the variant in the 3'UTR of complexin seems to be important for an exact control of CPLX2 expression in situations where it is needed.

We believe that this kind of research is essential to shed more light on the biological grounds of schizophrenia. It will enable us to get a better understanding of what is happening in the brain and ultimately lead to novel treatment approaches. 


\section{BIBLIOGRAPHY}

Addington J, Addington D (1999). Neurocognitive and social functioning in schizophrenia. Schizophr Bull 25, 173-182.

AmericanPsychiatricAssociation. Diagnostic and statistical manual of mental disorders: DSM-IV-TR. Washington: AmericanPsychiatricAssociation (2000)

Austin CP, Holder DJ, Ma L, Mixson LA, Caskey CT (1999). Mapping of hKCa3 to chromosome 1q21 and investigation of linkage of CAG repeat polymorphism to schizophrenia. Mol Psychiatry 4, 261-266.

Basso M, Giraudo S, Corpillo D, Bergamasco B, Lopiano L, Fasano M (2004). Proteome analysis of human substantia nigra in Parkinson's disease. Proteomics 4, 3943-3952.

Blank T, Nijholt I, Kye MJ, Radulovic J, Spiess J (2003). Small-conductance, Ca2+-activated K+ channel SK3 generates age-related memory and LTP deficits. Nat Neurosci 6, 911912.

Bond CT, Sprengel R, Bissonnette JM, Kaufmann WA, Pribnow D, Neelands T, Storck T, Baetscher M, Jerecic J, Maylie J, Knaus HG, Seeburg PH, Adelman JP (2000). Respiration and parturition affected by conditional overexpression of the $\mathrm{Ca} 2+-$ activated K+ channel subunit, SK3. Science 289, 1942-1946.

Brose N (2008a). For better or for worse: complexins regulate SNARE function and vesicle fusion. Traffic 9, 1403-1413.

Brose N (2008b). Altered complexin expression in psychiatric and neurological disorders: cause or consequence? Mol Cells 25, 7-19.

Brzustowicz LM, Hodgkinson KA, Chow EW, Honer WG, Bassett AS (2000). Location of a major susceptibility locus for familial schizophrenia on chromosome 1q21-q22. Science 288, 678-682.

Burdick KE, Gunawardane N, Woodberry K, Malhotra AK (2009). The role of general intelligence as an intermediate phenotype for neuropsychiatric disorders. Cogn Neuropsychiatry 14, 299-311.

Cai X, Liang CW, Muralidharan S, Kao JP, Tang CM, Thompson SM (2004). Unique roles of SK and Kv4.2 potassium channels in dendritic integration. Neuron 44, 351-364.

Cannon TD, Huttunen MO, Lonnqvist J, Tuulio-Henriksson A, Pirkola T, Glahn D, Finkelstein J, Hietanen M, Kaprio J, Koskenvuo M (2000). The inheritance of neuropsychological dysfunction in twins discordant for schizophrenia. Am J Hum Genet 67, 369-382.

Cardno AG, Bowen T, Guy CA, Jones LA, McCarthy G, Williams NM, Murphy KC, Spurlock G, Gray M, Sanders RD, Craddock N, McGuffin P, Owen MJ, O'Donovan MC (1999). CAG repeat length in the hKCa3 gene and symptom dimensions in schizophrenia. Biol Psychiatry 45, 1592-1596.

Carpenter WT, Jr. (2004). Clinical constructs and therapeutic discovery. Schizophr Res 72, 69-73.

Chandy KG, Fantino E, Wittekindt O, Kalman K, Tong LL, Ho TH, Gutman GA, Crocq MA, Ganguli R, Nimgaonkar V, Morris-Rosendahl DJ, Gargus JJ (1998). Isolation of a novel potassium channel gene hSKCa3 containing a polymorphic CAG repeat: a candidate for schizophrenia and bipolar disorder? Mol Psychiatry 3, 32-37.

Curtain R, Sundholm J, Lea R, Ovcaric M, MacMillan J, Griffiths L (2005). Association analysis of a highly polymorphic CAG Repeat in the human potassium channel gene KCNN3 and migraine susceptibility. BMC Med Genet 6, 32.

Drew CJ, Kyd RJ, Morton AJ (2007). Complexin 1 knockout mice exhibit marked deficits in social behaviours but appear to be cognitively normal. Hum Mol Genet 16, 2288-2305. 
Eastwood SL, Harrison PJ (2005). Decreased expression of vesicular glutamate transporter 1 and complexin II mRNAs in schizophrenia: further evidence for a synaptic pathology affecting glutamate neurons. Schizophr Res 73, 159-172.

Elvevag B, Goldberg TE (2000). Cognitive impairment in schizophrenia is the core of the disorder. Crit Rev Neurobiol 14, 1-21.

Erlenmeyer-Kimling L (2001). Early neurobehavioral deficits as phenotypic indicators of the schizophrenia genotype and predictors of later psychosis. Am J Med Genet 105, 23-24.

Faber ES (2009). Functions and modulation of neuronal SK channels. Cell Biochem Biophys 55, 127-139.

Faber ES, Delaney AJ, Sah P (2005). SK channels regulate excitatory synaptic transmission and plasticity in the lateral amygdala. Nat Neurosci 8, 635-641.

Figueroa KP, Chan P, Schols L, Tanner C, Riess O, Perlman SL, Geschwind DH, Pulst SM (2001). Association of moderate polyglutamine tract expansions in the slow calciumactivated potassium channel type 3 with ataxia. Arch Neurol 58, 1649-1653.

Fondon JW, 3rd, Hammock EA, Hannan AJ, King DG (2008). Simple sequence repeats: genetic modulators of brain function and behavior. Trends Neurosci 31, 328-334.

Freedman R (2003). Schizophrenia. $N$ Engl J Med 349, 1738-1749.

Gargus JJ (2006). Ion channel functional candidate genes in multigenic neuropsychiatric disease. Biol Psychiatry 60, 177-185.

Glatt SJ, Faraone SV, Tsuang MT (2003). CAG-repeat length in exon 1 of KCNN3 does not influence risk for schizophrenia or bipolar disorder: a meta-analysis of association studies. Am J Med Genet B Neuropsychiatr Genet 121B, 14-20.

Glynn D, Bortnick RA, Morton AJ (2003). Complexin II is essential for normal neurological function in mice. Hum Mol Genet 12, 2431-2448.

Glynn D, Reim K, Brose N, Morton AJ (2007). Depletion of Complexin II does not affect disease progression in a mouse model of Huntington's disease (HD); support for role for complexin II in behavioural pathology in a mouse model of HD. Brain Res Bull 72, 108-120.

Gold S, Arndt S, Nopoulos P, O'Leary DS, Andreasen NC (1999). Longitudinal study of cognitive function in first-episode and recent-onset schizophrenia. Am J Psychiatry 156, $1342-1348$.

Gottesman, II, Gould TD (2003). The endophenotype concept in psychiatry: etymology and strategic intentions. Am J Psychiatry 160, 636-645.

Green MF (1996). What are the functional consequences of neurocognitive deficits in schizophrenia? Am J Psychiatry 153, 321-330.

Guy CA, Bowen T, Williams N, Jones IR, McCandless F, McGuffin P, Owen MJ, Craddock N, O'Donovan MC (1999). No association between a polymorphic CAG repeat in the human potassium channel gene hKCa3 and bipolar disorder. Am J Med Genet 88, 5760.

Hammond RS, Bond CT, Strassmaier T, Ngo-Anh TJ, Adelman JP, Maylie J, Stackman RW (2006). Small-conductance Ca2+-activated K+ channel type 2 (SK2) modulates hippocampal learning, memory, and synaptic plasticity. J Neurosci 26, 1844-1853.

Huntwork S, Littleton JT (2007). A complexin fusion clamp regulates spontaneous neurotransmitter release and synaptic growth. Nat Neurosci 10, 1235-1237.

Hyman SE, Fenton WS (2003). Medicine. What are the right targets for psychopharmacology? Science 299, 350-351.

Jablensky A, Sartorius N, Ernberg G, Anker M, Korten A, Cooper JE, Day R, Bertelsen A (1992). Schizophrenia: manifestations, incidence and course in different cultures. A World Health Organization ten-country study. Psychol Med Monogr Suppl 20, 1-97.

Jacobsen JP, Redrobe JP, Hansen HH, Petersen S, Bond CT, Adelman JP, Mikkelsen JD, Mirza NR (2009). Selective cognitive deficits and reduced hippocampal brain-derived 
neurotrophic factor mRNA expression in small-conductance calcium-activated $\mathrm{K}+$ channel deficient mice. Neuroscience 163, 73-81.

Jacobsen JP, Weikop P, Hansen HH, Mikkelsen JD, Redrobe JP, Holst D, Bond CT, Adelman JP, Christophersen P, Mirza NR (2008). SK3 K+ channel-deficient mice have enhanced dopamine and serotonin release and altered emotional behaviors. Genes Brain Behav 7, 836-848.

Keefe RS, Eesley CE, Poe MP (2005). Defining a cognitive function decrement in schizophrenia. Biol Psychiatry 57, 688-691.

Keefe RS, Sweeney JA, Gu H, Hamer RM, Perkins DO, McEvoy JP, Lieberman JA (2007). Effects of olanzapine, quetiapine, and risperidone on neurocognitive function in early psychosis: a randomized, double-blind 52-week comparison. Am J Psychiatry 164, 1061-1071.

Kishi T, Ikeda M, Suzuki T, Kitajima T, Yamanouchi Y, Kinoshita Y, Ozaki N, Iwata N (2006). No association of complexin1 and complexin2 genes with schizophrenia in a Japanese population. Schizophr Res 82, 185-189.

Kohler M, Hirschberg B, Bond CT, Kinzie JM, Marrion NV, Maylie J, Adelman JP (1996). Small-conductance, calcium-activated potassium channels from mammalian brain. Science 273, 1709-1714.

Koronyo-Hamaoui M, Gak E, Stein D, Frisch A, Danziger Y, Leor S, Michaelovsky E, Laufer N, Carel C, Fennig S, Mimouni M, Apter A, Goldman B, Barkai G, Weizman A (2004). CAG repeat polymorphism within the KCNN3 gene is a significant contributor to susceptibility to anorexia nervosa: a case-control study of female patients and several ethnic groups in the Israeli Jewish population. Am J Med Genet B Neuropsychiatr Genet 131B, 76-80.

Kraepelin E. Dementia Praecox and Paraphrenia: Chicago Medical Book Co (1919)

Kuperberg G, Heckers S (2000). Schizophrenia and cognitive function. Curr Opin Neurobiol 10, 205-210.

Lee HJ, Song JY, Kim JW, Jin SY, Hong MS, Park JK, Chung JH, Shibata H, Fukumaki Y (2005). Association study of polymorphisms in synaptic vesicle-associated genes, SYN2 and CPLX2, with schizophrenia. Behav Brain Funct 1, 15.

McEvoy JP (2007). The costs of schizophrenia. J Clin Psychiatry 68 Suppl 14, 4-7.

McMahon HT, Missler M, Li C, Sudhof TC (1995). Complexins: cytosolic proteins that regulate SNAP receptor function. Cell 83, 111-119.

Mirnics K, Middleton FA, Lewis DA, Levitt P (2001). Analysis of complex brain disorders with gene expression microarrays: schizophrenia as a disease of the synapse. Trends Neurosci 24, 479-486.

Morton AJ, Faull RL, Edwardson JM (2001). Abnormalities in the synaptic vesicle fusion machinery in Huntington's disease. Brain Res Bull 56, 111-117.

Murray CJ, Lopez AD (1996). Evidence-based health policy--lessons from the Global Burden of Disease Study. Science 274, 740-743.

Obermair GJ, Kaufmann WA, Knaus HG, Flucher BE (2003). The small conductance Ca2+activated $\mathrm{K}+$ channel SK3 is localized in nerve terminals of excitatory synapses of cultured mouse hippocampal neurons. Eur J Neurosci 17, 721-731.

Orr HT, Zoghbi HY (2007). Trinucleotide repeat disorders. Annu Rev Neurosci 30, 575-621.

Owen MJ, O'Donovan MC, Harrison PJ (2005). Schizophrenia: a genetic disorder of the synapse? British Medical Journal 330, 158-159.

Palmer BW, Heaton RK, Paulsen JS, Kuck J, Braff D, Harris MJ, Zisook S, Jeste DV (1997). Is it possible to be schizophrenic yet neuropsychologically normal? Neuropsychology 11, 437-446. 
Pedarzani P, Stocker M (2008). Molecular and cellular basis of small--and intermediateconductance, calcium-activated potassium channel function in the brain. Cell Mol Life Sci 65, 3196-3217.

Rapoport JL, Addington AM, Frangou S, Psych MR (2005). The neurodevelopmental model of schizophrenia: update 2005. Mol Psychiatry 10, 434-449.

Reichenberg A, Weiser M, Rabinowitz J, Caspi A, Schmeidler J, Mark M, Kaplan Z, Davidson M (2002). A population-based cohort study of premorbid intellectual, language, and behavioral functioning in patients with schizophrenia, schizoaffective disorder, and nonpsychotic bipolar disorder. Am J Psychiatry 159, 2027-2035.

Reim K, Mansour M, Varoqueaux F, McMahon HT, Sudhof TC, Brose N, Rosenmund C (2001). Complexins regulate a late step in Ca2+-dependent neurotransmitter release. Cell 104, 71-81.

Reim K, Wegmeyer H, Brandstatter JH, Xue M, Rosenmund C, Dresbach T, Hofmann K, Brose N (2005). Structurally and functionally unique complexins at retinal ribbon synapses. J Cell Biol 169, 669-680.

Ribbe K, Friedrichs H, Begemann M, Grube S, Papiol S, Kästner A, Gerchen MF, Ackermann V, Tarami A, Treitz A, Flögel M, Adler L, Aldenhoff JB, Becker-Emner M, Becker T, Czernik A, Dose M, Folkerts H, Freese R, Gunther R, Herpertz S, Hesse D, Kruse G, Kunze H, Franz M, Lohrer F, Maier W, Mielke A, Müller-Isberner R, Oestereich C, Pajonk FG, Pollmacher T, Schneider U, Schwarz HJ, Kroner-Herwig B, HavemannReinecke U, Frahm J, Stühmer W, Falkai P, Brose N, Nave KA, Ehrenreich H (2010). The cross-sectional GRAS sample: A comprehensive phenotypical data collection of schizophrenic patients. BMC Psychiatry 10, 91.

Rimini R, Rimland JM, Terstappen GC (2000). Quantitative expression analysis of the small conductance calcium-activated potassium channels, SK1, SK2 and SK3, in human brain. Brain Res Mol Brain Res 85, 218-220.

Ritsner M, Modai I, Ziv H, Amir S, Halperin T, Weizman A, Navon R (2002). An association of CAG repeats at the KCNN3 locus with symptom dimensions of schizophrenia. Biol Psychiatry 51, 788-794.

Roncarati R, Di Chio M, Sava A, Terstappen GC, Fumagalli G (2001). Presynaptic localization of the small conductance calcium-activated potassium channel SK3 at the neuromuscular junction. Neuroscience 104, 253-262.

Rosa A, Fananas L, Cuesta MJ, Peralta V, Sham P (2002). 1q21-q22 locus is associated with susceptibility to the reality-distortion syndrome of schizophrenia spectrum disorders. Am J Med Genet 114, 516-518.

Sailer CA, Kaufmann WA, Marksteiner J, Knaus HG (2004). Comparative immunohistochemical distribution of three small-conductance Ca2+-activated potassium channel subunits, SK1, SK2, and SK3 in mouse brain. Mol Cell Neurosci 26, 458-469.

Sanders AR, Duan J, Levinson DF, Shi J, He D, Hou C, Burrell GJ, Rice JP, Nertney DA, Olincy A, Rozic P, Vinogradov S, Buccola NG, Mowry BJ, Freedman R, Amin F, Black DW, Silverman JM, Byerley WF, Crowe RR, Cloninger CR, Martinez M, Gejman PV (2008). No significant association of 14 candidate genes with schizophrenia in a large European ancestry sample: implications for psychiatric genetics. Am J Psychiatry 165, 497-506.

Sawa A, Snyder SH (2002). Schizophrenia: diverse approaches to a complex disease. Science 296, 692-695.

Sawada K, Barr AM, Nakamura M, Arima K, Young CE, Dwork AJ, Falkai P, Phillips AG, Honer WG (2005). Hippocampal complexin proteins and cognitive dysfunction in schizophrenia. Arch Gen Psychiatry 62, 263-272. 
Shepard PD, Bunney BS (1991). Repetitive firing properties of putative dopamine-containing neurons in vitro: regulation by an apamin-sensitive $\mathrm{Ca}(2+)$-activated $\mathrm{K}+$ conductance. Exp Brain Res 86, 141-150.

Snitz BE, Macdonald AW, 3rd, Carter CS (2006). Cognitive deficits in unaffected first-degree relatives of schizophrenia patients: a meta-analytic review of putative endophenotypes. Schizophr Bull 32, 179-194.

Stackman RW, Hammond RS, Linardatos E, Gerlach A, Maylie J, Adelman JP, Tzounopoulos T (2002). Small conductance Ca2+-activated $\mathrm{K}+$ channels modulate synaptic plasticity and memory encoding. J Neurosci 22, 10163-10171.

Stephan KE, Baldeweg T, Friston KJ (2006). Synaptic plasticity and dysconnection in schizophrenia. Biol Psychiatry 59, 929-939.

Sullivan PF, Kendler KS, Neale MC (2003). Schizophrenia as a complex trait: evidence from a meta-analysis of twin studies. Arch Gen Psychiatry 60, 1187-1192.

Tannenberg RK, Scott HL, Tannenberg AE, Dodd PR (2006). Selective loss of synaptic proteins in Alzheimer's disease: evidence for an increased severity with APOE varepsilon4. Neurochem Int 49, 631-639.

Tiwari AK, Zai CC, Muller DJ, Kennedy JL (2010). Genetics in schizophrenia: where are we and what next? Dialogues Clin Neurosci 12, 289-303.

Toulopoulou T, Goldberg TE, Mesa IR, Picchioni M, Rijsdijk F, Stahl D, Cherny SS, Sham P, Faraone SV, Tsuang M, Weinberger DR, Seidman LJ, Murray RM (2010). Impaired intellect and memory: a missing link between genetic risk and schizophrenia? Arch Gen Psychiatry 67, 905-913.

van Os J, Kenis G, Rutten BP (2010). The environment and schizophrenia. Nature 468, 203212.

Vijai J, Kapoor A, Ravishankar HM, Cherian PJ, Kuruttukulam G, Rajendran B, Sridharan R, Rangan G, Girija AS, Jayalakshmi S, Mohandas S, Mani KS, Radhakrishnan K, Anand A (2005). Protective and susceptibility effects of hSKCa3 allelic variants on juvenile myoclonic epilepsy. J Med Genet 42, 439-442.

Vincent JB, Paterson AD, Strong E, Petronis A, Kennedy JL (2000). The unstable trinucleotide repeat story of major psychosis. Am J Med Genet 97, 77-97.

Wittekindt O, Jauch A, Burgert E, Scharer L, Holtgreve-Grez H, Yvert G, Imbert G, Zimmer J, Hoehe MR, Macher JP, Chiaroni P, van Calker D, Crocq MA, Morris-Rosendahl DJ (1998). The human small conductance calcium-regulated potassium channel gene (hSKCa3) contains two CAG repeats in exon 1, is on chromosome 1q21.3, and shows a possible association with schizophrenia. Neurogenetics 1, 259-265.

Wolfart J, Neuhoff H, Franz O, Roeper J (2001). Differential expression of the smallconductance, calcium-activated potassium channel SK3 is critical for pacemaker control in dopaminergic midbrain neurons. J Neurosci 21, 3443-3456.

WorldHealthOrganization. The ICD-10 classification of mental and behavioural disorders: Clinical descriptions and diagnostic guidelines. Geneva: WHO (1992)

Yamauchi Y, Qin LH, Nishihara M, Sawada K, Kato K, Inoue S (2005). Vulnerability of synaptic plasticity in the complexin II knockout mouse to maternal deprivation stress. Brain Res 1056, 59-67. 


\section{APPENDIX}

\subsection{CURRICULUM VitAE}

\section{Personal data}

$\begin{aligned} \text { Name } & \text { Sabrina Grube } \\ \text { Birth name } & \text { Klaus } \\ \text { Date of birth } & \text { 23.12.1983 } \\ \text { Place of birth } & \text { Göttingen, Germany } \\ \text { Nationality } & \text { German } \\ \text { E-mail } & \text { sgrube@gmx.de }\end{aligned}$

\section{Education}

since April 2008 PhD student - Center for Systems Neuroscience, Graduate School for Neuroscience and Molecular Biosciences, Göttingen, Germany working with Professor Hannelore Ehrenreich at the Max Planck Institute of Experimental Medicine, Göttingen, Germany

March 2008 Diploma in Biochemistry, Leipniz University, Hannover, Germany June 2003 Abitur, Felix-Klein-Gymnasium, Göttingen, Germany 


\subsection{ACCEPTED CO-AUTHOR PUBLICATIONS}

Ribbe $\mathrm{K}^{*}$, Friedrichs $\mathrm{H}^{*}$, Begemann $\mathrm{M}^{*}$, Grube S, Papiol S, Kästner A, Gerchen MF, Ackermann V, Tarami A, Treitz A, Flögel M, Adler L, Aldenhoff B, Becker-Emner M, Becker T, Czernik A, Dose M, Folkerts H, Freese R, Günther R, Herpertz S, Hesse D, Kruse G, Kunze H, Löhrer F, Franz M, Maier W, Mielke A, Müller-Isberner R, Oestereich C, Pajonk F-G, Pollmächer T, Schneider U, Schwarz H-J, Kröner-Herwig B, HavemannReinecke U, Frahm J, Stühmer W, Falkai P, Brose N, Nave KA, Ehrenreich H (2010). The cross-sectional GRAS sample: A comprehensive phenotypical data collection of schizophrenic patients. BMC psychiatry 10, 91.

* Indicates equal contribution for the publication

Personal contribution:

I participated in the database set-up.

Papiol S, Begemann M, Rosenberger A, Friedrichs H, Ribbe K, Grube S, Schwab MH, Jahn H, Gunkel S, Benseler F, Nave KA, Ehrenreich H (2011). A phenotype-based genetic association study reveals the contribution of neuregulin1 gene variants to age of onset and positive symptom severity in schizophrenia. Am J Med Genet Part B [Epub ahead of print].

Personal contribution:

I organized the sample processing and assisted in genetic analysis. 


\title{
The cross-sectional GRAS sample: A comprehensive phenotypical data collection of schizophrenic patients
}

\author{
Katja Ribbe ${ }^{1 \dagger}$, Heidi Friedrichs ${ }^{1 \dagger}$, Martin Begemann ${ }^{1 \dagger}$, Sabrina Grube ${ }^{1}$, Sergi Papiol ${ }^{1,30}$, Anne Kästner ${ }^{1}$, Martin F Gerchen ${ }^{1}$, \\ Verena Ackermann ${ }^{1}$, Asieh Tarami ${ }^{1}$, Annika Treitz ${ }^{1}$, Marlene Flögel ${ }^{1}$, Lothar Adler ${ }^{2}$, Josef B Aldenhoff ${ }^{3}$, \\ Marianne Becker-Emner ${ }^{4}$, Thomas Becker ${ }^{5}$, Adelheid Czernik ${ }^{6}$, Matthias Dose ${ }^{7}$, Here Folkerts ${ }^{8}$, Roland Freese ${ }^{9}$, \\ Rolf Günther ${ }^{10}$, Sabine Herpertz ${ }^{11}$, Dirk Hesse ${ }^{12}$, Gunther Kruse ${ }^{13}$, Heinrich Kunze ${ }^{14}$, Michael Franz ${ }^{14}$, Frank Löhrer ${ }^{15}$, \\ Wolfgang Maier ${ }^{16}$, Andreas Mielke ${ }^{17}$, Rüdiger Müller-Isberner ${ }^{18}$, Cornelia Oestereich $^{19}$, Frank-Gerald Pajonk ${ }^{20}$, \\ Thomas Pollmächer ${ }^{21}$, Udo Schneider ${ }^{22}$, Hans-Joachim Schwarz ${ }^{23}$, Birgit Kröner-Herwig ${ }^{24}$, \\ Ursula Havemann-Reinecke ${ }^{25,30}$, Jens Frahm ${ }^{26,30,31}$, Walter Stühmer ${ }^{27,30,31}$, Peter Falkai ${ }^{25,30,31}$, Nils Brose ${ }^{28,30,31}$, \\ Klaus-Armin Nave ${ }^{29,30,31}$, Hannelore Ehrenreich ${ }^{1,30,31^{*}}$
}

\begin{abstract}
Background: Schizophrenia is the collective term for an exclusively clinically diagnosed, heterogeneous group of mental disorders with still obscure biological roots. Based on the assumption that valuable information about relevant genetic and environmental disease mechanisms can be obtained by association studies on patient cohorts of $\geq 1000$ patients, if performed on detailed clinical datasets and quantifiable biological readouts, we generated a new schizophrenia data base, the GRAS (Göttingen Research Association for Schizophrenia) data collection. GRAS is the necessary ground to study genetic causes of the schizophrenic phenotype in a 'phenotype-based genetic association study' (PGAS). This approach is different from and complementary to the genome-wide association studies (GWAS) on schizophrenia.
\end{abstract}

Methods: For this purpose, 1085 patients were recruited between 2005 and 2010 by an invariable team of traveling investigators in a cross-sectional field study that comprised 23 German psychiatric hospitals. Additionally, chart records and discharge letters of all patients were collected.

Results: The corresponding dataset extracted and presented in form of an overview here, comprises biographic information, disease history, medication including side effects, and results of comprehensive cross-sectional psychopathological, neuropsychological, and neurological examinations. With $>3000$ data points per schizophrenic subject, this data base of living patients, who are also accessible for follow-up studies, provides a wide-ranging and standardized phenotype characterization of as yet unprecedented detail.

Conclusions: The GRAS data base will serve as prerequisite for PGAS, a novel approach to better understanding 'the schizophrenias' through exploring the contribution of genetic variation to the schizophrenic phenotypes.

\section{Background}

Schizophrenia is a devastating brain disease that affects approximately $1 \%$ of the population across cultures [1]. The diagnosis of schizophrenia or - perhaps more correctly - of 'the schizophrenias' is still purely clinical, requiring the

\footnotetext{
* Correspondence: ehrenreich@em.mpg.de

+ Contributed equally

'Division of Clinical Neuroscience, Max Planck Institute of Experimental Medicine, Göttingen, Germany

Full list of author information is available at the end of the article
}

coincident presence of symptoms as listed in the leading classification systems, DSM-IV and ICD-10 [2,3].

Notably, one of the core symptoms of schizophrenia, namely cognitive deficits, from mild impairments to full-blown dementia, has not yet been considered in these classifications. Biologically, schizophrenia is a 'mixed bag' of diseases that undoubtedly have a strong genetic root. Family studies exploring relative risk of schizophrenia have led to estimates of heritability of about $64-88 \%$ [4,5]. Monozygotic twin studies showing

\section{() Biomed Central}

C 2010 Ribbe et al; licensee BioMed Central Ltd. This is an Open Access article distributed under the terms of the Creative Commons Attribution License (http://creativecommons.org/licenses/by/2.0), which permits unrestricted use, distribution, and reproduction in any medium, provided the original work is properly cited. 
concordance rates of $41-65 \%$ [6,7] indicate a considerable amount of non-genetic causes, in the following referred to as 'environmental factors'. Already in the middle of the twentieth century, schizophrenia was seen as a 'polygenetic' disease [8] and, indeed, in numerous genetic studies since, ranging from segregation or linkage analyses, genome scans and large association studies, no major 'schizophrenia gene' has been identified [9]. Even recent genome-wide association studies (GWAS) on schizophrenia confirm that several distinct loci are associated with the disease. These studies concentrated on endpoint diagnosis and found odds ratios for single markers in different genomic regions ranging from 0.68 to 6.01 [10], essentially underlining the fact that - across ethnicities in most cases these genotypes do not contribute more to the disease than a slightly increased probability.

We hypothesize that an interplay of multiple causative factors, perhaps thousands of potential combinations of genes/genetic markers and an array of different environmental risks, leads to the development of 'the schizophrenias', as schematically illustrated in Figure 1. There may be cases with a critical genetic load already present without need of additional external co-factors, however, in most individuals, an interaction of a certain genetic predisposition with environmental co-factors is apparently required for disease onset. In fact, not too much of an overlap may exist between genetic risk factors from one schizophrenic patient to an unrelated other schizophrenic individual, explaining why it is basically impossible to find common risk genes of schizophrenia with appreciable odds ratios. One GRAS working hypothesis is that in the overwhelming majority of cases, schizophrenia is the result of a 'combination of unfortunate genotypes'.

If along the lines of traditional human genetics all attempts to define schizophrenia as a 'classical' genetic disease have largely failed, how can we learn more about the contribution of genes/genotypes to the disease phenotype? Rather than searching by GWAS for yet other schizophrenia risk genes, we designed an alternative and widely complementary approach, termed PGAS (phenotype-based genetic association study), in order to

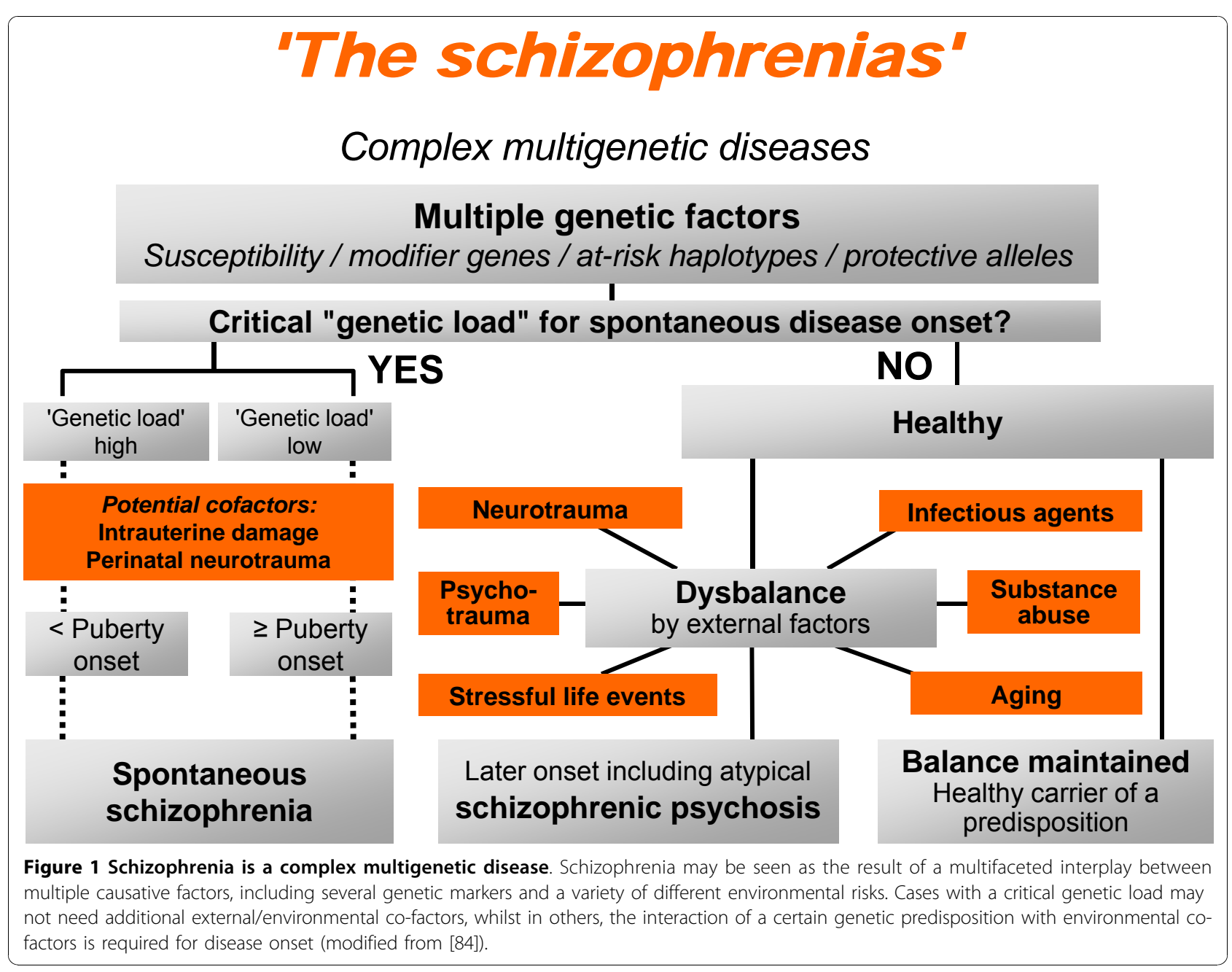


explore the contribution of certain genes/genetic markers to the schizophrenic phenotype. To launch PGAS, we had to establish a comprehensive phenotypical data base of schizophrenic patients, the GRAS (Göttingen Research Association for Schizophrenia) data collection. Very recently, we have been able to demonstrate proofof-concept for the PGAS approach [[11], and Grube et al: Calcium-activated potassium channels as regulators of cognitive performance in schizophrenia, submitted].

Large data bases of schizophrenic patients have been instigated for decades to perform linkage/family studies, treatment trials, genetic or epidemiological studies applying either a cross-sectional or a longitudinal design (e.g. [12-20]). However, for the above introduced PGAS approach, another type of data base is required, and only few of the existing data banks are suited for phenotypical analyses. An example is the 'Clinical Antipsychotic Trial of Intervention Effectiveness (CATIE)', originally set up as a treatment study comparing a first generation antipsychotic drug with several second generation antipsychotics in a multisite randomized doubleblind trial $[17,21]$. The huge amount of data accumulated in the frame of this trial serves now also for GWAS and genotype-phenotype association studies [22-25]. Disadvantages may be that the CATIE data were collected by different examiners in 57 US sites and that comprehensive data for phenotypical analyses are only available for subsamples of the originally included 1493 patients. Another example of a large data base with considerable phenotypical power is the 'Australian Schizophrenia Research Bank (ASRB)’ [26]. ASRB operates to collect, store and distribute linked clinical, cognitive, neuroimaging and genetic data from a large sample of patients with schizophrenia (at present nearly 500) and healthy controls (almost 300) [27,28]).

The present paper has been designed (1) to introduce the GRAS data collection, set up as prerequisite and platform for PGAS; (2) to exemplify on some selected areas of interest the potential of phenotypical readouts derived from the GRAS data collection and their internal consistency; (3) to provide a first panel of epidemiological data as a 'side harvest' of this data base; and (4) to enable interested researchers worldwide to initiate scientific collaborations based on this data base.

\section{Methods \\ Ethics}

The GRAS data collection has been approved by the ethical committee of the Georg-August-University of Göttingen (master committee) as well as by the respective local regulatories/ethical committees of all collaborating centers (Table 1). The distribution of the centers over Germany together with information on the numbers of recruited patients per center is presented in Figure 2.

\section{GRAS patients}

From September 2005 to July 2008, a total of 1071 patients were examined by the GRAS team of traveling investigators after giving written informed consent, own and/or authorized legal representatives. Since then, lowrate steady state recruitment has been ongoing, among others to build up a new cohort for replicate analyses of genotype-phenotype associations. As of July 2010, 1085 patients have been entered into the data base. They were examined in different settings: 348 (32.1\%) as outpatients, $474(43.7 \%)$ as inpatients in psychiatric hospitals, $189(17.4 \%)$ as residents in sheltered homes, 54 (5\%) as patients in specific forensic units, and $20(1.8 \%)$ as day clinic patients. Inclusion criteria were (1) confirmed or suspected diagnosis of schizophrenia or schizoaffective disorder according to DSM-IV and (2) at least some ability to cooperate. Recruitment efficiency over the core travel/field study time from 2005 to 2008 and patient flow are shown in Figures $3 \mathrm{a}$ and $3 \mathrm{~b}$. Of the 1085 patients entered into the data base, a total of 1037 fulfilled the diagnosis of schizophrenia or schizoaffective disorder. For 48 patients the diagnosis of schizophrenia could not be ultimately confirmed upon careful re-check and follow-up. Of the schizophrenic patients, 96\% completed the GRAS assessment whereas about 4\% dropped out during the examination. Almost all patients agreed to be re-contacted for potential follow-up studies, only $1.5 \%$ were either lost to follow-up (present address unknown or deceased) or did not give consent to be contacted again.

\section{Healthy control subjects}

(1) For genetic analyses, control subjects, who gave written informed consent, were voluntary blood donors, recruited by the Department of Transfusion Medicine at the Georg-August-University of Göttingen according to national guidelines for blood donation. As such, they widely fulfill health criteria, ensured by a broad predonation screening process containing standardized questionnaires, interviews, hemoglobin, blood pressure, pulse, and body temperature determinations. Of the total of 2265 subjects, $57.5 \%$ are male $(\mathrm{n}=1303)$ and $42.5 \%$ female $(n=962)$. The average age is $33.8 \pm 12.2$ years, with a range from 18 to 69 years. Participation as healthy controls for the GRAS sample was anonymous, with information restricted to age, gender, blood donor health state and ethnicity. Comparable to the patient population (Table 2), almost all control subjects were of European Caucasian descent (Caucasian 97.8\%; other ethnicities $2 \%$; unknown $0.2 \%$ ). (2) For selected cognitive measures and olfactory testing, 103 additional healthy volunteers were recruited as control subjects (matched with respect to age, gender, and smoking habits). These healthy controls include $67.0 \%$ male $(\mathrm{n}=69)$ and $33.0 \%$ 
Table 1 GRAS data collection manual: Table of contents

\begin{tabular}{|c|c|c|}
\hline category & content & reference in the paper \\
\hline \multirow[t]{12}{*}{$\begin{array}{l}\text { legal documents/ethical requirements } \\
\text { patient history }\end{array}$} & $\begin{array}{l}\text { patient information, informed consent form, confidentiality form, and others... } \\
\text { general information (age, sex, ethnicity,...) }\end{array}$ & $\rightarrow$ table 2 \\
\hline & education/employment & $\rightarrow$ table 2 \\
\hline & living situation & $\rightarrow$ table 2 \\
\hline & legal history & \\
\hline & medication including side effects & $\rightarrow$ table 4 \\
\hline & medical history & \\
\hline & family history & \\
\hline & global quality of life ${ }^{a}$ & $\rightarrow$ table 2 and figure 6 \\
\hline & birth history/traumatic brain injury & \\
\hline & stressful life events & \\
\hline & suicidal thoughts/suicide attempts & \\
\hline & hospitalization history & $\rightarrow$ table 2 and figure 6 \\
\hline \multirow[t]{4}{*}{ clinical interviews/ratings } & parts of SCID-I: addiction, anxiety, affective disorders, psychotic disorders ${ }^{* b}$ & \\
\hline & Positive and Negative Syndrome Scale* (PANSS) ${ }^{c}$ & $\rightarrow$ table 2 and figure 6 \\
\hline & Clinical Global Impression* $(C G I)^{d}$ & $\rightarrow$ table 2 and figure 6 \\
\hline & Global Assessment of Functioning* $(G A F)^{e}$ & $\rightarrow$ table 2 and figure 6 \\
\hline \multirow[t]{3}{*}{ questionnaires } & State-Trait-Anxiety-Inventory* $\left(\right.$ STAl) ${ }^{\dagger}$ & $\rightarrow$ table 2 and figure 6 \\
\hline & Brief Symptom Inventory* $(\mathrm{BSI})^{9}$ & $\rightarrow$ table 2 and figure 6 \\
\hline & Toronto Alexithymia Scale* (TAS) $^{\text {h }}$ & $\rightarrow$ table 2 \\
\hline \multirow[t]{8}{*}{ cognitive tests } & premorbid IQ (MWT-B) $)^{i, j}$ & $\rightarrow$ table 3 and figure 7 \\
\hline & reasoning $(\text { LPS-3) })^{k}$ & $\rightarrow$ table 3 and figure 7 \\
\hline & letter-number-span (BZT)' & $\rightarrow$ table 3 and figure 7 \\
\hline & finger dotting and tapping ${ }^{m}$ & $\rightarrow$ table 3 and figure 7 \\
\hline & trail making tests (TMT-A and TMT-B) ${ }^{n}$ & $\rightarrow$ table 3 and figure 7 \\
\hline & verbal fluency $(\mathrm{DT} / \mathrm{RWT})^{\mathrm{o}, \mathrm{p}}$ & \\
\hline & digit-symbol test $(Z S T)^{q}$ & $\rightarrow$ table 3 and figure 7 \\
\hline & verbal memory* $(V L M T)^{r}$ & $\rightarrow$ table 3 and figure 7 \\
\hline \multirow[t]{10}{*}{ physical examination } & Testbatterie zur Aufmerksamkeitsprüfung (TAP) & $\rightarrow$ table 3 and figure 7 \\
\hline & general physical examination & \\
\hline & Cambridge Neurological Inventory $(\mathrm{CNI})^{\mathrm{t}}$ & $\rightarrow$ table 5 and figure 8 \\
\hline & Contralateral Co-Movement Test (COMO) ${ }^{\mathrm{u}}$ & \\
\hline & Barnes Akathisia Rating Scale (BARS) ${ }^{\vee}$ & $\rightarrow$ figure 8 \\
\hline & Simpson-Angus Scale (SAS) ${ }^{\mathrm{w}}$ & $\rightarrow$ figure 8 \\
\hline & Tardive Dyskinesia Rating Scale (TDRS) ${ }^{x}$ & $\rightarrow$ figure 8 \\
\hline & Abnormal Involuntary Movement Scale (AIMS) & $\rightarrow$ figure 8 \\
\hline & odor testing $(\mathrm{ORNI} \text { Test })^{z}$ & \\
\hline & blood sampling (DNA, serum) & \\
\hline
\end{tabular}

${ }^{*}$ questionnaires and cognitive tests in respective German versions

${ }^{a}$ Based on a visual analogue scale (Krampe H, Bartels C, Victorson D, Enders CK, Beaumont J, Cella D, Ehrenreich H: The influence of personality factors on disease progression and health-related quality of life in people with ALS. Amyotroph Lateral Scler 2008, 9:99-107). 'bittchen H-U, Zaudig, M. and Fydrich, T.: SKID-I (Strukturiertes Klinisches Interview für DSM-IV; Achse I: Psychische Störungen). Göttingen: Hogrefe; 1997. 'Kay SR, Fiszbein A, Opler LA: The positive and negative syndrome scale (PANSS) for schizophrenia. Schizophr Bull 1987, 13(2):261-276. ${ }^{\text {d} G u y ~ W: ~ C l i n i c a l ~ G l o b a l ~ I m p r e s s i o n ~(C G I) . ~ I n ~ E C D E U ~ A s s e s s m e n t ~ m a n u a l ~ f o r ~ p s y c h o p h a r m a c o l o g y, ~ r e v i s e d ~ N a t i o n a l ~ I n s t i t u e ~ o f ~}$ Mental Health. Rockville, MD; 1976. ${ }^{\mathrm{e}}$ AmericanPsychiatricAssociation: Diagnostic and statistical manual of mental disorders, 4th edition (DSM-IV). Washington, DC: American Psychiatric Press; 1994. fLaux L, Glanzmann P, Schaffner P, Spielberger CD: Das State-Trait-Angstinventar (STAI). Weinheim: Beltz; 1981. 9Franke GH: Brief Symptom Inventory (BSI). Goettingen: Beltz; 2000. ' $K$ Kupfer J, Brosig B, Braehler E: Toronto Alexithymie-Skala-26 (TAS-26). Goettingen: Hogrefe; 2001. 'Lehrl S, Triebig G, Fischer B: Multiple choice vocabulary test MWT as a valid and short test to estimate premorbid intelligence. Acta Neurol Scand 1995, 91(5):335-345. 'Lehrl S: Mehrfach-Wortschatz-Intelligenztest MWT-B. Balingen: Spitta Verlag; 1999. ${ }^{k}$ Horn W: Leistungsprüfsystem (LPS). 2 edition. Goettingen: Hogrefe; 1983. 'Gold JM, Carpenter C, Randolph C, Goldberg TE, Weinberger DR: Auditory working memory and Wisconsin Card Sorting Test performance in schizophrenia. Arch Gen Psychiatry 1997, 54(2):159-165. mChapman RL: The MacQuarrie test for mechanical ability. Psychometrika 1948, 13(3):175-179. "War-Department: Army Individual Test Battery. Manual of directions and scoring. Washington, D.C.: War Department, Adjutant General's Office; 1944. ${ }^{\circ}$ Kessler J, Denzler P, Markowitsch HJ: Demenz-Test (DT). Göttingen: Hogrefe; $1999 .{ }^{\mathrm{P} A s c h e n b r e n n e r ~ S, ~ T u c h a ~ O, ~ L a n g e ~ K W: ~ D e r ~ R e g e n s b u r g e r ~}$

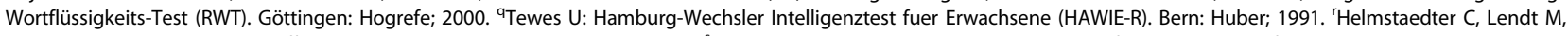

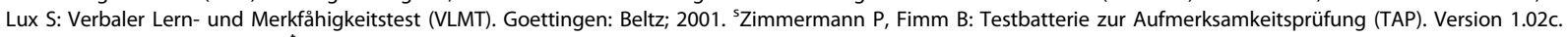
Herzogenrath: PSYTEST; 1993. 'Chen EY, Shapleske J, Luque R, McKenna PJ, Hodges JR, Calloway SP, Hymas NF, Dening TR, Berrios GE: The Cambridge Neurological Inventory: a clinical instrument for assessment of soft neurological signs in psychiatric patients. Psychiatry Res 1995, 56(2):183-204. "Bartels C, Mertens N, Hofer S, Merboldt KD, Dietrich J, Frahm J, Ehrenreich H: Callosal dysfunction in amyotrophic lateral sclerosis correlates with diffusion tensor imaging of the central motor system. Neuromuscul Disord 2008, 18 (5):398-407. 'Barnes TR: The Barnes Akathisia Rating Scale - revisited. J Psychopharmacol 2003, 17(4):365-370. "Simpson GM, Angus JW: A rating scale for extrapyramidal side effects. Acta Psychiatr Scand Suppl 1970, 212:11-19. ${ }^{\times}$Simpson GM, Lee JH, Zoubok B, Gardos G: A rating scale for tardive dyskinesia. Psychopharmacology (Berl) 1979, 64 (2):171-179. ' Guy W: Abnormal involuntary movement scale (AIMS). In ECDEU Assessment manual for psychopharmacology, revised National Institute of Mental Health. Rockville, MD; 1976. ${ }^{\mathrm{z}}$ ORNI Test (Odor Recognition, Naming and Interpretation Test; developed for the purpose of odor testing in schizophrenics; manuscript in preparation) 


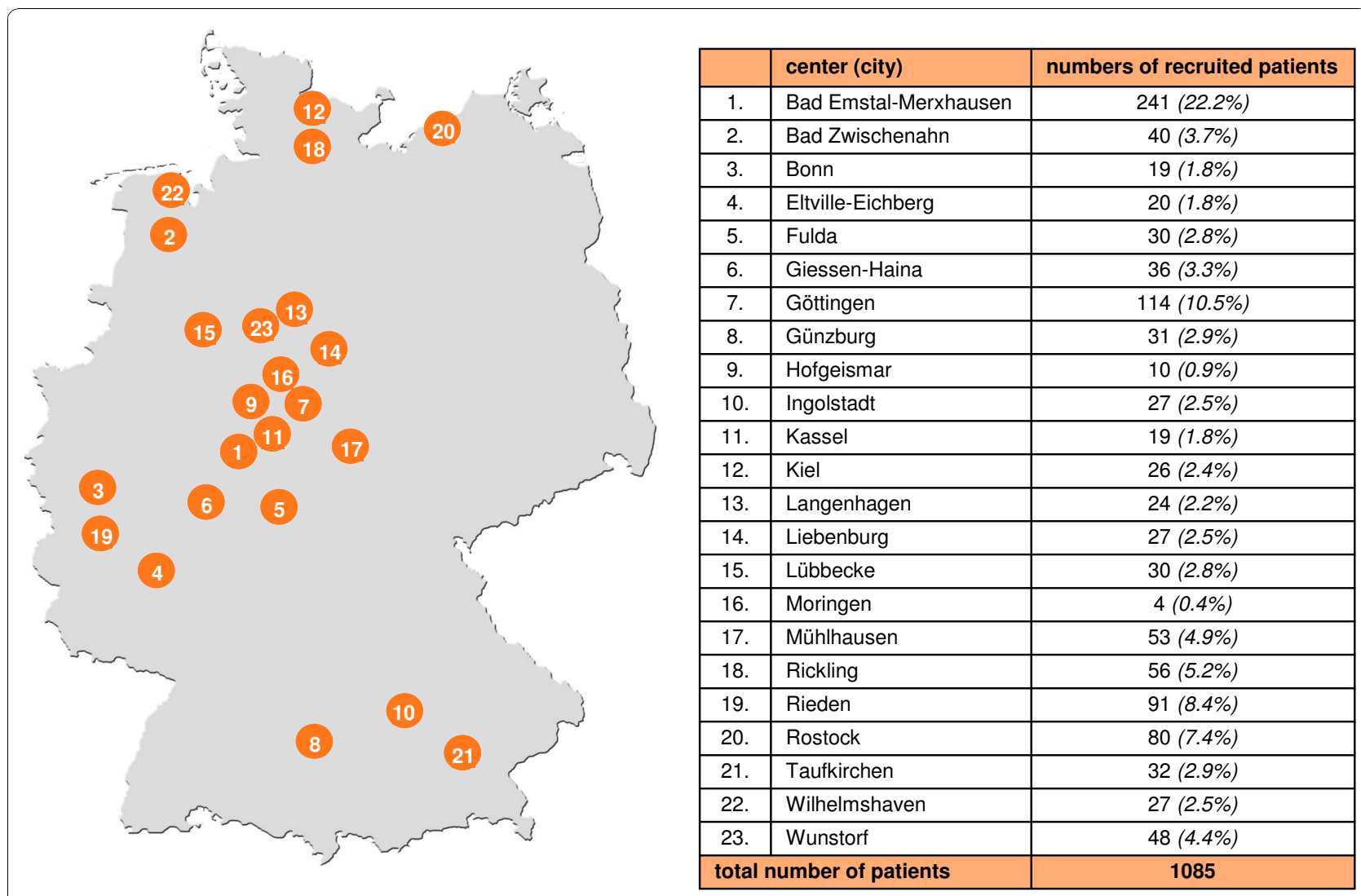

Figure 2 Collaborating centers and patient numbers. Map of Germany displaying the locations of all 23 collaborating centers that were visited by an invariable team of traveling investigators. The table next to the map provides numbers of patients examined in each center. Some centers were visited more than once.

$(\mathrm{n}=34)$ female subjects with an average age of $39.02 \pm$ 13.87 years, ranging from 18 to 71 years.

\section{Traveling team}

The GRAS team of traveling investigators consisted of 1 trained psychiatrist and neurologist, 3 psychologists and 4 medical doctors/last year medical students. All investigators had continuous training and calibration sessions to ensure the highest possible agreement on diagnoses and other judgments as well as a low interrater variability regarding the instruments applied. Patient contacts were usually prepared by colleagues/personnel in the respective collaborating psychiatric centers (Figure 2) to make the work of the travel team as efficient as possible.

\section{The GRAS manual}

A standardized procedure for examination of the patients has been arranged with the GRAS manual, composed for the purpose of the GRAS data collection. Table 1 presents its contents, including established instruments, such as clinical interviews/ratings, questionnaires, cognitive and neurological tests [2,29-53].

\section{GRAS operating procedure}

The GRAS data base operating procedure leading from the large set of raw data provided by the travel team to the data bank with its several-fold controlled and verified data points is illustrated in Figure 4. Already during the time when the travel team examined patients all over Germany, a team of psychologists started to work on the development of the GRAS data base, integrating the raw data to ultimately result in over 3000 phenotypic data points per patient (total of over 3.000000 data points at present in the data collection) (Figure 5). Most importantly, the chart records/medical reports of all patients were carefully screened, missing records identified and, in numerous, sometimes extensive and repeated, telephone and written conversations, missing psychiatric discharge letters of every single patient organized. After careful study and pre-processing of raw data and chart records, the confirmation of the diagnoses, determination of age of onset of the disease and prodrome as well as other essential readouts were achieved by meticulous consensus decisions. 

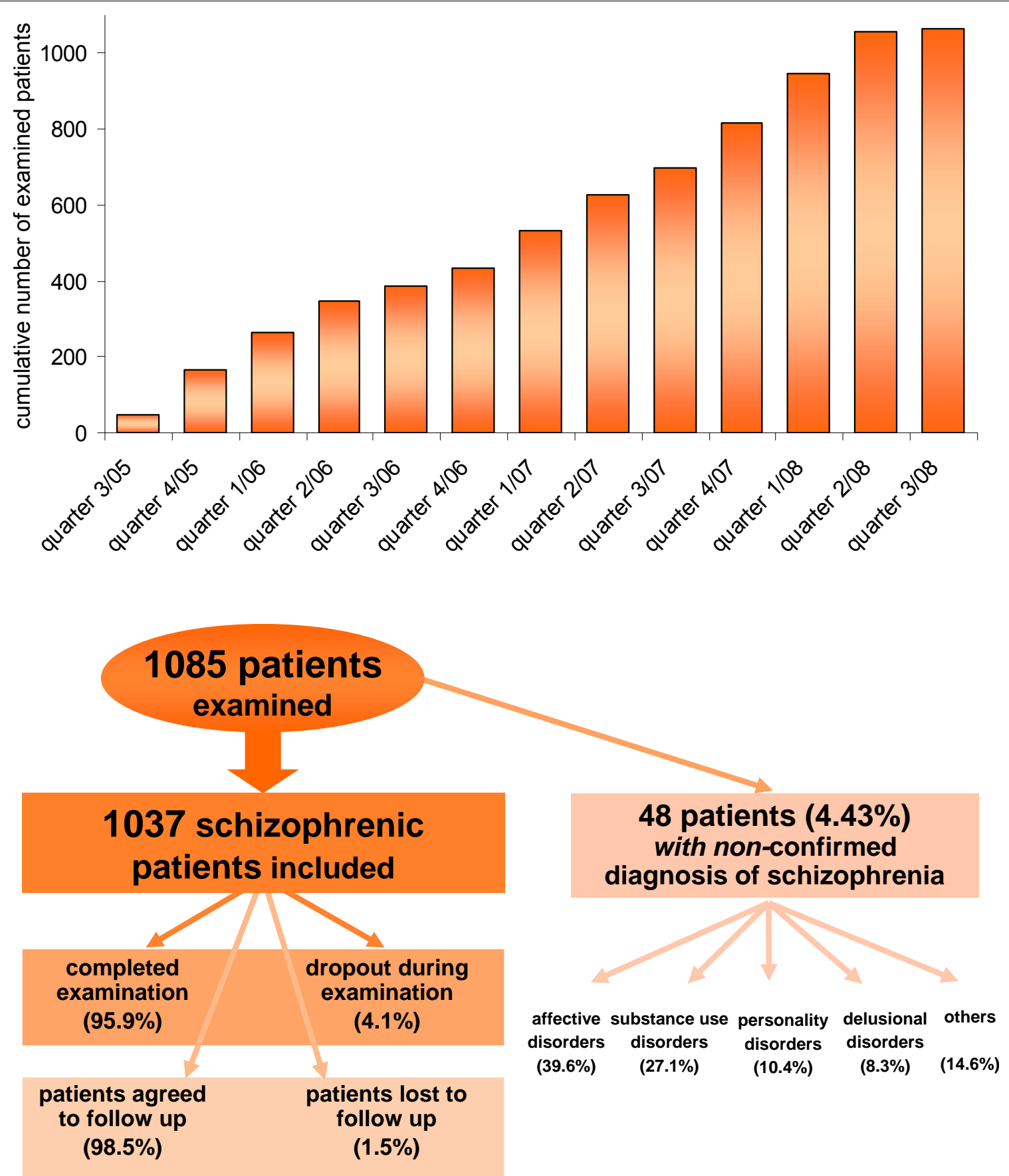

Figure 3 Patient recruitment and flow: (a) Recruitment efficiency 2005 - 2008. Cumulative numbers of recruited patients per quarter of the year are shown in bar graphs. Note that steady-state recruitment is ongoing. (b) Patient flow. Of 1085 patients examined, the diagnosis of schizophrenia or schizoaffective disorder could not be confirmed for 48. Instead, alternative diagnoses had to be given.

\section{Statistical analyses}

For the establishment of the data base and for basic statistical analyses of the data, SPSS for Windows version 17.0 [54] was used. Comparisons of men and women in terms of sociodemographic and clinical picture as well as neurological examination were assessed using either
Mann-Whitney-U or Chi-square test. Prior to correlation and regression analyses, selected metric phenotypic variables were standardized by Blom transformation [55]. The Blom transformation is a probate transformation into ranks and the resulting standardized values are normally distributed with zero mean and variance one. 


\begin{tabular}{|c|c|c|c|c|c|c|c|c|c|c|c|c|c|c|c|}
\hline & & \multicolumn{4}{|c|}{ total } & \multicolumn{4}{|c|}{ men } & \multicolumn{4}{|c|}{ women } & \multicolumn{2}{|c|}{ statistics } \\
\hline & & $\mathrm{N}$ & $\%$ & mean (sd) & median & $\mathbf{N}$ & $\%$ & mean (sd) & median & $\mathbf{N}$ & $\%$ & mean (sd) & median & $\chi^{2 / z}$ & $\mathbf{P}$ \\
\hline \multicolumn{16}{|l|}{ sociodemographics } \\
\hline total n & & 1037 & 100 & & & 693 & 100 & & & 344 & 100 & & & & \\
\hline age (in years) & & & & $\begin{array}{r}39.52 \\
(12.56)\end{array}$ & 39.05 & & & $\begin{array}{r}37.57 \\
(11.97)\end{array}$ & 36.67 & & & $\begin{array}{r}43.46 \\
(12.80)\end{array}$ & 42.85 & $Z=-6.980$ & $0.001^{<}$ \\
\hline $\begin{array}{l}\text { education } \\
\text { (in years) }\end{array}$ & & & & $11.94(3.37)$ & 12.00 & & & 11.71 (3.34) & 12.00 & & & $12.42(3.39)$ & 12.00 & $Z=-2.714$ & $0.007^{*}$ \\
\hline \multirow[t]{4}{*}{ ethnicity: } & caucasian & 992 & 95.66 & & & 661 & 95.38 & & & 331 & 96.20 & & & & \\
\hline & african & 7 & 0.68 & & & 6 & 0.87 & & & 1 & 0.30 & & & & \\
\hline & mixed & 10 & 0.96 & & & 7 & 1.01 & & & 3 & 0.90 & & & $\chi^{2}=1.202$ & 0.753 \\
\hline & unknown & 28 & 2.70 & & & 19 & 2.74 & & & 9 & 2.60 & & & & \\
\hline \multirow[t]{3}{*}{ native tongue: } & German & 902 & 86.98 & & & 591 & 85.71 & & & 311 & 90.67 & & & & \\
\hline & bi-lingual German & 46 & 4.44 & & & 38 & 4.33 & & & 8 & 1.46 & & & $\chi^{2}=6.899$ & $0.032^{*}$ \\
\hline & other & 89 & 8.58 & & & 64 & 9.96 & & & 25 & 7.87 & & & & \\
\hline \multirow[t]{5}{*}{ marital status: } & single & 748 & 72.13 & & & 575 & 82.97 & & & 173 & 50.44 & & & & \\
\hline & married & 129 & 12.44 & & & 48 & 6.93 & & & 81 & 23.32 & & & & \\
\hline & divorced & 124 & 11.96 & & & 57 & 8.23 & & & 67 & 19.53 & & & $\begin{array}{r}\chi^{2}= \\
121.516\end{array}$ & $0.001^{<}$ \\
\hline & widowed & 13 & 1.25 & & & 3 & 0.43 & & & 10 & 2.92 & & & & \\
\hline & unknown & 23 & 2.22 & & & 10 & 1.44 & & & 13 & 3.79 & & & & \\
\hline \multirow[t]{9}{*}{ living situation: } & alone & 292 & 28.16 & & & 201 & 29.00 & & & 91 & 26.45 & & & & \\
\hline & alone with children & 17 & 1.64 & & & 0 & 0 & & & 17 & 4.94 & & & & \\
\hline & with partner ( \pm children) & 137 & 13.20 & & & 50 & 7.22 & & & 87 & 25.29 & & & & \\
\hline & With parents & 157 & 15.14 & & & 121 & 17.46 & & & 36 & 10.47 & & & & \\
\hline & $\begin{array}{l}\text { with others (family members, } \\
\text { friends) }\end{array}$ & 71 & 6.85 & & & 53 & 7.65 & & & 18 & 5.23 & & & $\begin{array}{r}\chi^{2}= \\
116.823\end{array}$ & $0.001^{<}$ \\
\hline & sheltered home & 282 & 27.19 & & & 212 & 30.59 & & & 70 & 20.35 & & & & \\
\hline & forensic hospital & 54 & 5.21 & & & 43 & 6.20 & & & 11 & 3.20 & & & & \\
\hline & homeless & 4 & 0.39 & & & 4 & 0.58 & & & 0 & 0 & & & & \\
\hline & unknown & 23 & 2.22 & & & 9 & 1.30 & & & 14 & 4.07 & & & & \\
\hline \multicolumn{16}{|l|}{ clinical picture } \\
\hline diagnosis: & $\begin{array}{l}\text { classical schizophrenias } \\
\text { schizoaffective disorders }\end{array}$ & $\begin{array}{l}852 \\
185\end{array}$ & $\begin{array}{l}82.16 \\
17.84\end{array}$ & & & $\begin{array}{r}615 \\
78\end{array}$ & $\begin{array}{l}88.74 \\
11.26\end{array}$ & & & $\begin{array}{l}237 \\
107\end{array}$ & $\begin{array}{l}68.90 \\
31.10\end{array}$ & & & $\begin{array}{r}\chi^{2}= \\
61.794\end{array}$ & $0.001^{<}$ \\
\hline $\begin{array}{l}\text { age of onset of first psychotic } \\
\text { episode }\end{array}$ & & & & $25.75(8.81)$ & 23.00 & & & $24.49(7.71)$ & 22.00 & & & $\begin{array}{r}28.28 \\
(10.23)\end{array}$ & 26.00 & $Z=-5.705$ & $0.001^{<}$ \\
\hline duration of disease (in years) & & & & $\begin{array}{r}13.23 \\
(10.71)\end{array}$ & 10.87 & & & $\begin{array}{r}12.57 \\
(10.38)\end{array}$ & 10.16 & & & $\begin{array}{r}14.54 \\
(11.24)\end{array}$ & 13.02 & $Z=-2.600$ & $0.009^{*}$ \\
\hline $\begin{array}{l}\text { hospitalization (number of } \\
\text { inpatient stays) }\end{array}$ & & & & $8.60(9.76)$ & 6.00 & & & $8.49(9.95)$ & 5.00 & & & $8.83(9.38)$ & 6.00 & $Z=-0.727$ & 0.467 \\
\hline
\end{tabular}


Table 2: GRAS sample description (Continued)

\begin{tabular}{|c|c|c|c|c|c|c|c|c|c|}
\hline chlorpromazine equivalents & & $\begin{array}{r}687.36 \\
(696.85)\end{array}$ & 499.98 & $\begin{array}{r}706.67 \\
(668.43)\end{array}$ & 520.00 & $\begin{array}{r}648.35 \\
(750.50)\end{array}$ & 450.00 & $Z=-2.428$ & $0.015^{*}$ \\
\hline \multirow[t]{4}{*}{ PANSS ${ }^{\mathrm{a}}$ : } & positive symptoms & $13.76(6.32)$ & 12.00 & $13.94(6.16)$ & 12.00 & $13.92(6.64)$ & 12.00 & $Z=-0.130$ & 0.990 \\
\hline & negative symptoms & $18.23(7.85)$ & 17.00 & $18.14(7.57)$ & 17.00 & $18.11(8.44)$ & 17.00 & 0.886 & 0.376 \\
\hline & general psychiatric symptoms & $\begin{array}{r}33.73 \\
(11.83)\end{array}$ & 32.00 & $\begin{array}{r}33.37 \\
(11.31)\end{array}$ & 32.00 & $\begin{array}{r}34.50 \\
(12.81)\end{array}$ & 33.00 & -0.886 & 0.376 \\
\hline & total score & $\begin{array}{r}65.64 \\
(23.40)\end{array}$ & 63.00 & $\begin{array}{r}65.32 \\
(22.41)\end{array}$ & 63.00 & $\begin{array}{r}66.31 \\
(25.37)\end{array}$ & 62.00 & -0.025 & 0.980 \\
\hline Clinical Global Impression scale & & 5.57 & 6.00 & $5.57(1.03)$ & 6.00 & $5.57(1.18)$ & 6.00 & $Z=-0.121$ & 0.894 \\
\hline Global Assessment of Functioning ${ }^{c}$ & & $45.76(0.68)$ & 45.00 & $\begin{array}{r}45.60 \\
(16.30)\end{array}$ & 45.00 & $\begin{array}{r}46.09 \\
(19.11)\end{array}$ & 45.00 & $Z=-0.323$ & 0.747 \\
\hline global quality of life ${ }^{d}$ & & $5.41(2.37)$ & 5.00 & $5.43(2.31)$ & 5.00 & $5.38(2.49)$ & 5.00 & $Z=-0.378$ & 0.705 \\
\hline Brief Symptom Inventory e: & general severity index & $0.88(0.68)$ & 0.71 & $0.87(0.66)$ & 0.71 & $0.92(0.72)$ & 0.71 & $Z=-0.687$ & 0.492 \\
\hline \multirow[t]{2}{*}{ State-Trait-Anxiety Inventory ${ }^{f}$ : } & state anxiety & $\begin{array}{r}43.54 \\
(10.89)\end{array}$ & 43.00 & $\begin{array}{r}43.48 \\
(10.45)\end{array}$ & 43.00 & $\begin{array}{r}43.65 \\
(11.79)\end{array}$ & 43.00 & $Z=-0.121$ & 0.904 \\
\hline & trait anxiety & $\begin{array}{r}44.96 \\
(11.34)\end{array}$ & 45.00 & $\begin{array}{r}44.67 \\
(11.09)\end{array}$ & 45.00 & $\begin{array}{r}45.56 \\
(11.82)\end{array}$ & 46.00 & -0.983 & 0.326 \\
\hline Toronto Alexithymia Scale ${ }^{\mathrm{g}}$ & & $2.59(0.56)$ & 2.61 & $2.58(0.54)$ & 2.55 & $2.60(0.60)$ & 2.66 & $Z=-0.607$ & 0.544 \\
\hline
\end{tabular}

aKay SR, Fiszbein A, Opler LA: The positive and negative syndrome scale (PANSS) for schizophrenia. Schizophr Bull1987,13(2):261-276. 'buy W: Clinical Global Impressions (CGI). In ECDEU Assessment manual for psychopharmacology, revised Nationallinstitue of Mental Health. Rockville, MD; 1976. 'AmericanPsychiatricAssociation: Diagnostic and statistical manual of mental disorders, 4th edition (DSM-IV). Washington, DC:
American Psychiatric Press; 1994. ${ }^{d}$ Based on a visual analogue scale (Krampe H, Bartels C, Victorson D, Enders CK, Beaumont J, Cella D, Ehrenreich H: The influence of personality factors on disease progression and

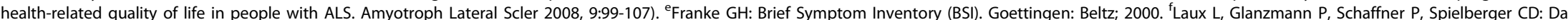

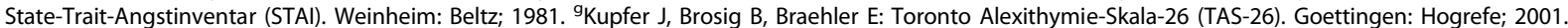




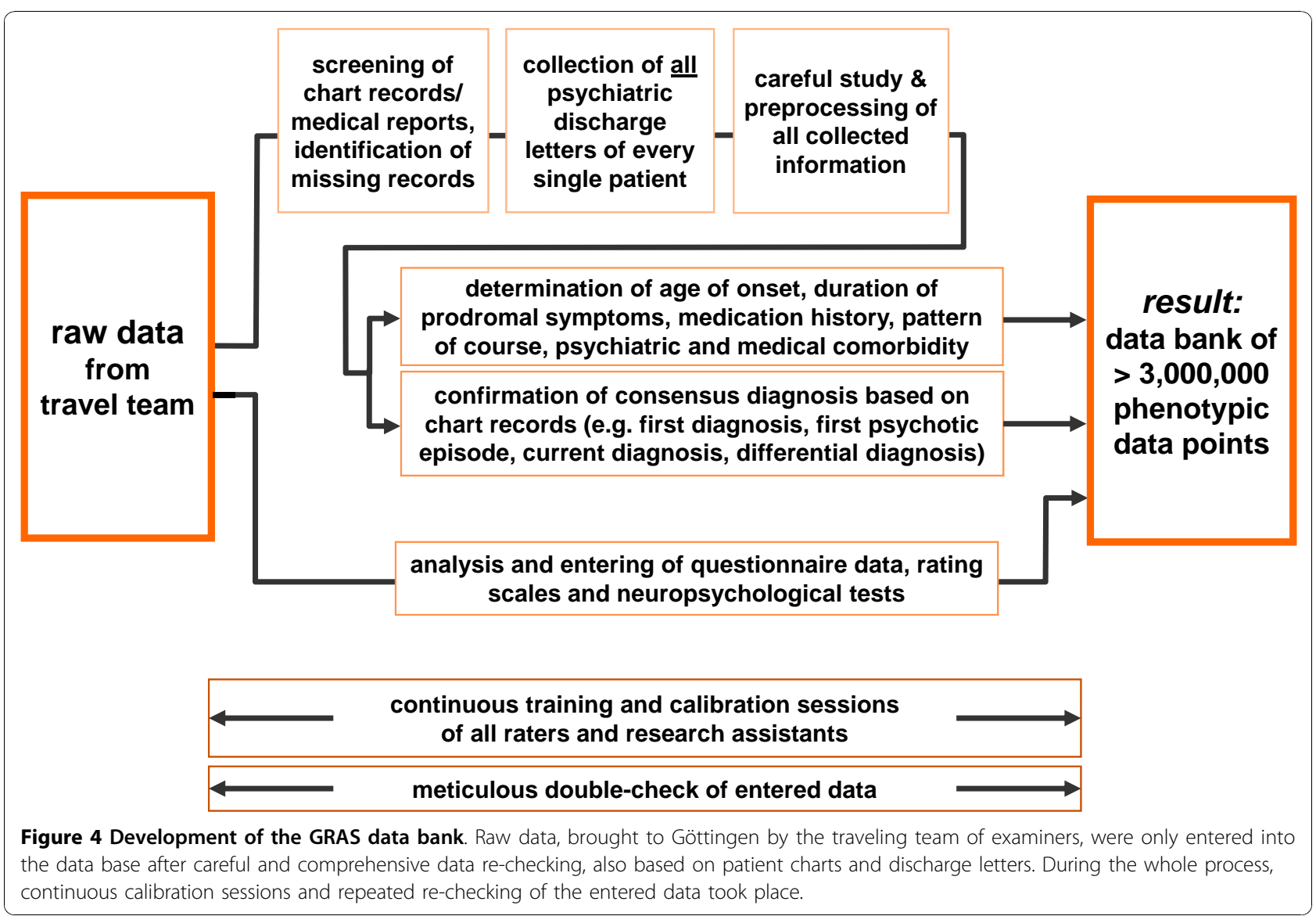

Comparisons of men and women in terms of cognitive performance were assessed by analyses of covariance, using age, duration of disease, years of education and chlorpromazine equivalents as covariates. For all intercorrelation patterns, correlations of the particular target variables were assessed using Pearson product-moment correlation. Cronbach's alpha coefficient was determined for estimation of internal consistency of the target variables within a defined intercorrelation pattern. Multiple regression analyses using the enter method were conducted to evaluate the contribution of selected disease related variables (duration of disease, positive symptoms, negative symptoms, catatonic signs and chlorpromazine equivalents) to 3 dependent variables: basic cognition/ fine motor functions, cognitive functions and global functioning (GAF) [2]. The dependent variables basic cognition/fine motor functions and cognitive functions are both composite score variables. The basic cognition/ fine motor function score comprises alertness (TAP), dotting and tapping (Cronbach's alpha $=.801)[39,46]$ and the cognition score consists of reasoning (LPS3), 2 processing speed measures (TMT-A and digit-symbol test, ZST), executive functions (TMT-B), working memory (BZT), verbal learning \& memory (VLMT) and divided attention (TAP) $[37,38,41,44-46]$ (Cronbach's alpha $=.869$ ). For both scores, a Cronbach's alpha $>.80$ indicates a high internal consistency as prerequisite for integrating several distinct items into one score. Multiple regression analyses were conducted for the total sample and separated for men and women.

\section{Results}

Biographic and clinical data

The GRAS data collection comprises presently (as of August 2010) 1037 patients with confirmed diagnosis of schizophrenia (82.2\%) or schizoaffective disorder (17.8\%). A total of 693 men and 344 women fulfilled the respective diagnostic requirements of DSM-IV. Table 2 provides a sample description, both total and separated for male and female patients, with respect to sociodemographic data and clinical picture. There are some differences between genders in the GRAS sample: Women are older, less single, have more years of education, more diagnoses of schizoaffective disorders, longer duration of disease, later age of onset of first psychotic episode and lower doses of antipsychotics. However, regarding determinants of the clinical picture, e.g. PANSS scores [30], genders do not differ significantly. 
neuropsychology / cognition: speed of processing, attention / vigilance, working memory, verbal learning, reasoning / problem solving (executive functioning), motor function, crystalline / fluid intelligence...

medication history: type, combination, dose of antipsychotic medication during disease course, side effects...

hospitalization: number and duration of psychiatric inpatient stays and forensic stays...

birth complications: prolonged birth, asphyxia, premature birth...

\section{physical examination:}

minor abnormalities, comorbidity..

neuro- and psychotrauma: cerebral contusion, loss of consciousness, abuse during childhood, migration... neurological examination: neurological standard exam, soft signs, odor testing, saccadic eye movements...

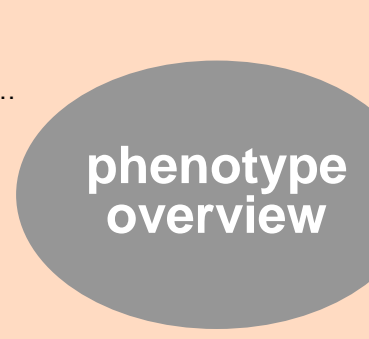

disease history: age of onset, duration of prodromal symptoms, first diagnosis, first psychotic episode...

\footnotetext{
psychopathology: psychiatric ratings, subjective symptoms, course, diagnostic categories, hallucination and delusion phenomena...
}

psychiatric comorbidity: anxiety, depression, mania substance abuse, e.g. alcohol, cannabis..

Figure 5 Phenotype overview. Various different domains covered by the GRAS data collection are displayed. These domains will also deliver the basis for further sophistication of phenotypical readouts.

An intercorrelation pattern of selected clinical readouts, obtained by (1) clinical ratings and (2) self-ratings of the patients and complemented by (3) 'objective data', in this case medication and hospitalization, is presented in Figure 6. The Cronbach's alpha of .753 suggests that items derived from the 3 different perspectives harmonize well. Whereas patient ratings of quality of life and state anxiety (STAI) [32] are only weakly correlated with professional clinical ratings and objective data, the patients' self-estimated symptom burden as measured with the BSI [33] shows moderate to good correlation.

\section{Cognition}

For the ongoing/planned genetic analyses, not only the clinical picture with its schizophrenia-typical positive and negative symptoms, but particularly cognition plays an important role. The cognitive tests applied in the GRAS data collection show an intercorrelation pattern that further underlines quality and internal consistency of the data obtained by the invariable team of investigators (Figure 7). Table 3 represents the cognitive performance data of the complete GRAS sample in the respective domains. In addition, the performance level of men and women is given as well as - for comparison - available normative data of healthy individuals. Since for dotting and tapping [39], no normative data were available in the literature, the values shown in Table 3 were obtained from the healthy GRAS control population for cognitive measures $(n=103$; see patients and methods).

Comparing cognitive performance of schizophrenic men and women, analyses of covariance have been conducted, with age, duration of disease, years of education and chlorpromazine equivalents as covariates, which revealed significant gender differences in discrete cognitive domains. Men performed better in reasoning $(\mathrm{F}=$ 17.62, $\mathrm{p}<.001)$, alertness $(\mathrm{F}=28.30, \mathrm{p}<.001$ for reaction time and $\mathrm{F}=10.39, \mathrm{p}=.001$ for lapses), and divided attention $(\mathrm{F}=14.07 \mathrm{p}<.001$ for reaction time and $\mathrm{F}=$ $22.12, \mathrm{p}<.001$ for lapses). In contrast, female schizophrenic patients were superior in verbal memory tasks $(\mathrm{F}=12.38, \mathrm{p}<.001)$ and digit symbol test $(\mathrm{F}=19.24, \mathrm{p}$ $<.001)$. With respect to normative data obtained from healthy controls, cognitive data of all schizophrenic patients are in the lower normal range (percentile rank $=16$ for digit symbol test) or even below (percentile 


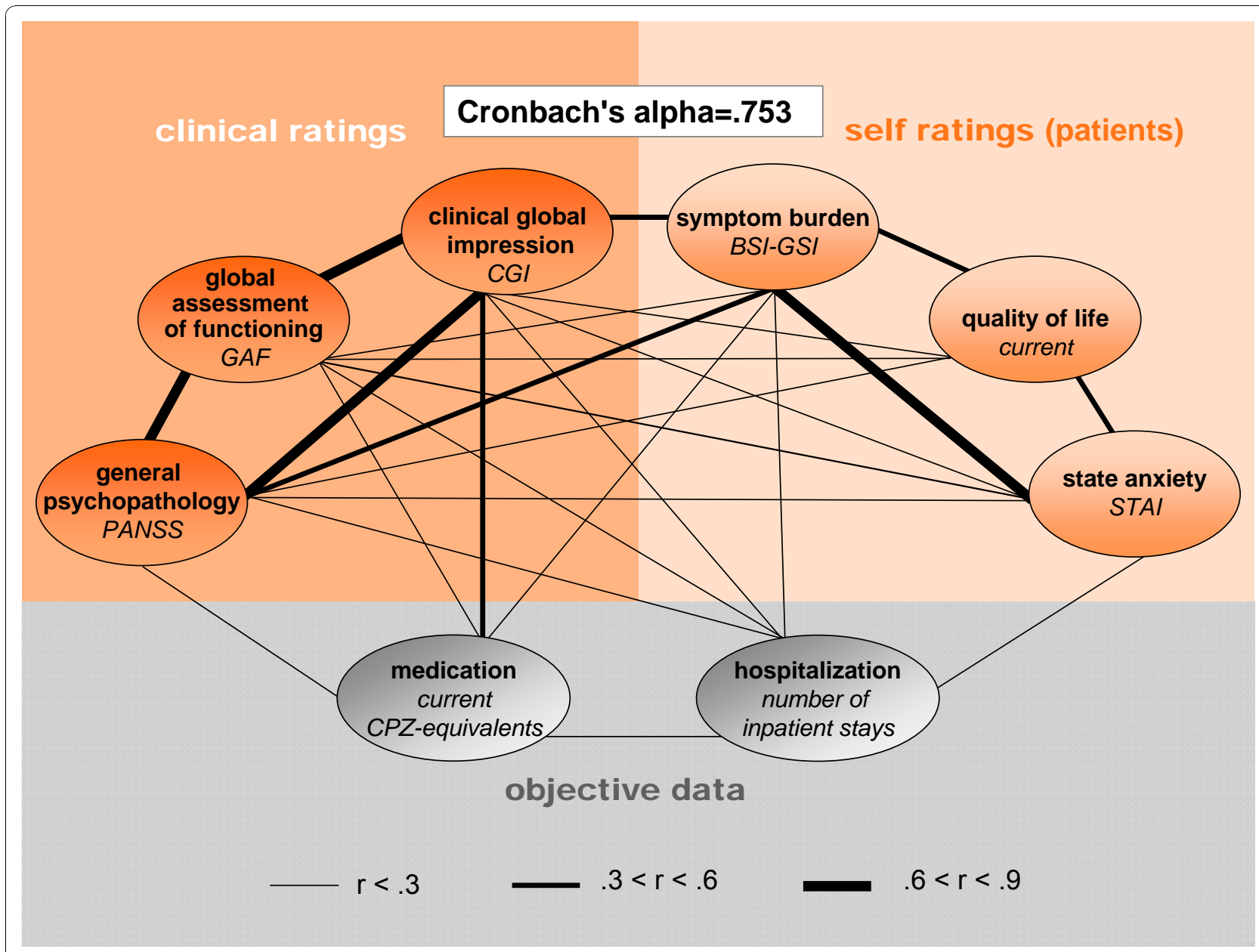

Figure 6 Clinical intercorrelation pattern. Correlations between measures of the clinical picture derived from different approaches: Patient self-ratings, clinical rater judgement and 'objective data'. Thickness of the lines represents the strength of correlation between two measures; only significant correlations are displayed. Note the strong internal consistency expressed by a Cronbach's alpha of .753.

ranks 10 for verbal memory, TMT-A, TMT-B, alertness and divided attention). Only for reasoning (LPS) [37] and premorbid intelligence (MWT-B) [36], schizophrenic subjects lie in the average range (percentile ranks of 31 and 43.5 respectively).

\section{Antipsychotic medication and side effects}

Another important feature of schizophrenic patients that may influence their every-day functioning and performance, and result in a considerable number of side effects, is their antipsychotic medication. The GRAS data collection contains information on type, dose, duration of medication and drugs prescribed over the years. The mean dose of present antipsychotic medication of the whole GRAS population, expressed as chlorpromazine equivalents [56] amounts to 687.36 ( \pm 696.85$)$. Chlorpromazine equivalents in male are significantly higher as compared to female patients (Table 2). An overview of self-reported side effects of current antipsychotic medication in the
GRAS sample, again sorted by gender, is given in Table 4. Of the 1037 patients with confirmed diagnosis of schizophrenia/schizoaffective disorder, 24 were presently not on antipsychotic drugs, whilst for 1 patient the current medication was unknown. Of the remaining 1012 patients who currently receive antipsychotic medication (16.5\% first generation antipsychotics, $54.1 \%$ second generation antipsychotics and $29.4 \%$ mixed) and were all explicitly interviewed regarding medication side effects, only 423 reported any. The discrepancy between side effects measured versus side effects based on patients' reports becomes obvious when considering for instance the number of patients with clear extrapyramidal symptoms: A total of 335 subjects measured by Simpson-Angus Scale (mean score >.3) [50] contrasts only 117 patients selfreporting extrapyramidal complaints. External rating of extrapyramidal side effects in the GRAS population was comprehensively performed, utilizing a number of respective instruments which all showed significant 


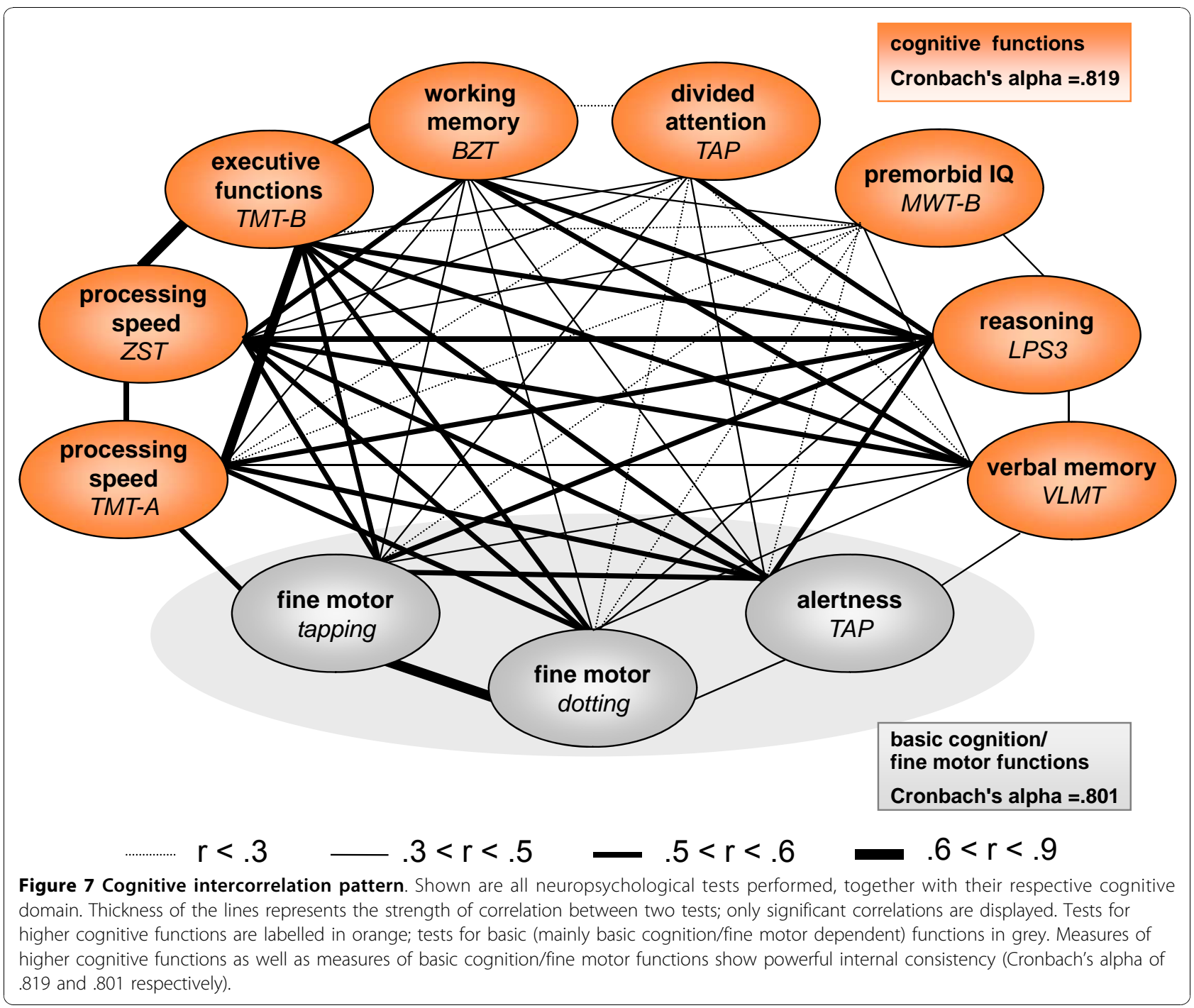

intercorrelation (Figure 8) [47,49-52,57]. A composite score of the 6 Blom transformed scales, used for testing potential gender effects, yielded no significant differences in extrapyramidal symptoms in men versus women $(\mathrm{Z}=$ -0.022, $\mathrm{p}=0.982$ ).

\section{Neurological symptoms}

Similar to cognitive readouts, evaluation of inherent neurological symptoms in the schizophrenic patient population are of tremendous interest, not only for understanding the contribution of particular genes/ genetic markers and/or environmental factors to the schizophrenic phenotype but also for estimating the impact of potential neurological comorbidities. Table 5 provides an overview of neurological symptoms based on the Cambridge Neurological Inventory (CNI) [47]. Only in the subscale 'Failure to suppress inappropriate response', significant differences between men and women $(\mathrm{Z}=-3.175, \mathrm{p}=0.001)$ became evident. Women were less able to hold respective responses back, e.g. to blink with one eye, leaving the other eye open, or to perform saccadic eye movements without moving the head.

\section{Prediction of functioning}

In order to delineate the influence of disease on functioning in the GRAS sample, multiple regression analyses have been employed. These procedures assessed the contribution of 5 disease-related variables, i.e. duration of disease, PANSS positive and negative scores [30], catatonic signs [47], and dose of antipsychotic medication, to 3 dependent performance variables: (a) basic cognition/fine motor functions, (b) cognitive performance and (c) global functioning (Table 6). Regarding basic cognition/fine motor function, multiple regression analysis revealed a significant model accounting for 
Table 3 Cognitive performance of GRAS patients. For comparison, normative data are presented wherever available2.

\begin{tabular}{|c|c|c|c|c|c|c|c|c|c|c|c|c|c|c|}
\hline & \multicolumn{3}{|c|}{ men } & \multicolumn{3}{|c|}{ women } & \multicolumn{2}{|c|}{ ANCOVA } & \multicolumn{3}{|c|}{ total } & \multicolumn{3}{|c|}{$\begin{array}{c}\text { normative data (PR) or } \\
\text { mean } \\
\text { sample } \\
\text { values of healthy } \\
\text { controls }\end{array}$} \\
\hline & $\mathbf{N}$ & $\begin{array}{l}\text { mean } \\
\text { (sd) }\end{array}$ & median & $\mathrm{N}$ & $\begin{array}{l}\text { mean } \\
\text { (sd) }\end{array}$ & median & $\mathbf{F}$ & $\mathbf{p}$ & $\mathrm{N}$ & $\begin{array}{l}\text { mean } \\
\text { (sd) }\end{array}$ & median & $\mathrm{N}$ & $\begin{array}{l}\text { PR } \\
\text { (Percentile } \\
\text { Rank) }\end{array}$ & $\begin{array}{l}\text { mean } \\
\text { (sd) }\end{array}$ \\
\hline reasoning (LPS) & 663 & $\begin{array}{l}21.26 \\
(6.70)\end{array}$ & 22.00 & 324 & $\begin{array}{l}18.79 \\
(6.31)\end{array}$ & 18.00 & 17.62 & $\stackrel{<}{.001^{*}}$ & 987 & $\begin{array}{l}20.45 \\
(6.67)\end{array}$ & 21.00 & $1556^{a}$ & 31 & - \\
\hline working memory (BZT) & 627 & $\begin{array}{l}13.24 \\
(3.79)\end{array}$ & 14.00 & 312 & $\begin{array}{l}12.62 \\
(3.91)\end{array}$ & 13.00 & 1.20 & .274 & 939 & $\begin{array}{l}13.03 \\
(3.84)\end{array}$ & 13.00 & $30^{b}$ & - & $\begin{array}{l}15.70 \\
(2.6)\end{array}$ \\
\hline $\begin{array}{l}\text { executive functions } \\
\left({ }^{(T M T-B)^{\circ}}\right.\end{array}$ & 631 & $\begin{array}{l}131.42 \\
(104.21)\end{array}$ & 99.00 & 307 & $\begin{array}{l}147.65 \\
(121.09)\end{array}$ & 108.00 & 0.00 & .956 & 938 & $\begin{array}{l}136.73 \\
(110.22)\end{array}$ & 100.00 & $24^{c}$ & 10 & $\begin{array}{l}71.5 \\
(31.07)\end{array}$ \\
\hline $\begin{array}{l}\text { verbal memory } \\
\text { (VLMT) }\end{array}$ & 602 & $\begin{array}{l}41.15 \\
(12.63)\end{array}$ & 41.00 & 302 & $\begin{array}{l}42.68 \\
(13.02)\end{array}$ & 42.00 & 12.38 & $\begin{array}{l}< \\
.001^{*}\end{array}$ & 904 & $\begin{array}{l}41.66 \\
(12.78)\end{array}$ & 42.00 & $89^{d}$ & 10 & $\begin{array}{l}52.39 \\
(7.87)\end{array}$ \\
\hline premorbid IQ ${ }^{1)}(\mathrm{MWT}-\mathrm{B})$ & 613 & $\begin{array}{l}25.96 \\
(6.22)\end{array}$ & 27.00 & 311 & $\begin{array}{l}26.21 \\
(6.13)\end{array}$ & 27.00 & 0.69 & .405 & 924 & $\begin{array}{l}26.04 \\
(6.19)\end{array}$ & 27.00 & $1952^{e}$ & 43.5 & - \\
\hline \multicolumn{15}{|l|}{$\begin{array}{l}\text { divided attention } \\
\left(\text { TAP }^{\circ}\right.\end{array}$} \\
\hline reaction time & 651 & $\begin{array}{l}759.67 \\
(114.25)\end{array}$ & 743.43 & 308 & $\begin{array}{l}805.16 \\
(150.99)\end{array}$ & 780.04 & 14.07 & $\begin{array}{l}< \\
.001^{*}\end{array}$ & 959 & $\begin{array}{l}774.28 \\
(128.89)\end{array}$ & 755.05 & $200^{f}$ & 8 & - \\
\hline lapses & & $\begin{array}{l}3.35 \\
(7.15)\end{array}$ & 1.00 & & $\begin{array}{l}6.41 \\
(13.18)\end{array}$ & 2.00 & 22.12 & $\begin{array}{l}< \\
.001^{*}\end{array}$ & & $\begin{array}{l}4.33 \\
(9.62)\end{array}$ & 1.00 & & & \\
\hline \multicolumn{15}{|l|}{ processing speed } \\
\hline $\begin{array}{l}\text { trail making test } A \\
(\text { TMT-A })^{\circ}\end{array}$ & 676 & $\begin{array}{l}49.18 \\
(35.22)\end{array}$ & 40.00 & 332 & $\begin{array}{l}55.32 \\
(42.22)\end{array}$ & 43.00 & 0.17 & .683 & 1008 & $\begin{array}{l}51.20 \\
(37.76)\end{array}$ & 41.00 & $24^{c}$ & $<5$ & $\begin{array}{l}33.04 \\
(7.89)\end{array}$ \\
\hline $\begin{array}{l}\text { digit-symbol test } \\
\text { (ZST) }\end{array}$ & 674 & $\begin{array}{l}37.46 \\
(12.58)\end{array}$ & 37.00 & 329 & $\begin{array}{l}38.58 \\
(14.14)\end{array}$ & 39.00 & 19.24 & $\dot{<}_{.001 *}$ & 1003 & $\begin{array}{l}37.83 \\
(13.12)\end{array}$ & 38.00 & $200^{9}$ & 16 & - \\
\hline \multicolumn{15}{|l|}{$\begin{array}{l}\text { basic cognition/fine } \\
\text { motor function }\end{array}$} \\
\hline \multicolumn{15}{|l|}{ alertness $(\mathrm{TAP})^{\circ}$} \\
\hline reaction time & 665 & $\begin{array}{l}319.62 \\
(116.13)\end{array}$ & 284.08 & 326 & $\begin{array}{l}379.11 \\
(161.80)\end{array}$ & 328.04 & 28.30 & $\begin{array}{l}< \\
.001^{*}\end{array}$ & 991 & $\begin{array}{l}339.19 \\
(135.73)\end{array}$ & 298.41 & $200^{f}$ & 10 & - \\
\hline lapses & & $\begin{array}{l}0.52 \\
(2.04)\end{array}$ & 0.00 & & $\begin{array}{l}1.18 \\
(3.57)\end{array}$ & 0.00 & 10.39 & $.001^{*}$ & & $\begin{array}{l}0.73 \\
(2.66)\end{array}$ & 0.00 & & & \\
\hline dotting & 673 & $\begin{array}{l}46.10 \\
(13.08)\end{array}$ & 46.00 & 320 & $\begin{array}{l}45.36 \\
(14.96)\end{array}$ & 46.00 & 1.62 & .203 & 993 & $\begin{array}{l}45.86 \\
(13.71)\end{array}$ & 46.00 & $103^{h}$ & - & $\begin{array}{l}63.24 \\
(11.03)\end{array}$ \\
\hline tapping & 671 & $\begin{array}{l}29.01 \\
(8.57)\end{array}$ & 29.00 & 319 & $\begin{array}{l}27.58 \\
(9.00)\end{array}$ & 27.00 & 0.76 & .783 & 990 & $\begin{array}{l}28.55 \\
(8.73)\end{array}$ & 28.00 & $103^{h}$ & - & $\begin{array}{l}37.63 \\
(7.04)\end{array}$ \\
\hline
\end{tabular}

- Higher scores reflect better performance, except for TMT-A, TMT-B, Alertness and Divided Attention (TAP)

* For statistical comparison (ANCOVA) between men and women values are corrected for age, duration of disease, chlorpromazine equivalents and years of education (except MWT-B).

1) Non-native and non-bilingual German speaking patients are excluded ( $n=89$ ).

2) Percentile ranks $(P R)<15$ indicate that the mean or the median of the total sample is below average in comparison to a normative sample.

${ }^{a}$ Horn W: Leistungsprüfsystem (LPS). 2 edition. Goettingen: Hogrefe; 1983. ${ }^{b}$ Gold JM, Carpenter C, Randolph C, Goldberg TE, Weinberger DR: Auditory working memory and Wisconsin Card Sorting Test performance in schizophrenia. Arch Gen Psychiatry 1997, 54(2):159-165. 'Perianez JA, Rios-Lago M, Rodriguez-Sanchez JM, Adrover-Roig D, Sanchez-Cubillo I, Crespo-Facorro B, Quemada Jl, Barcelo F: Trail Making Test in traumatic brain injury, schizophrenia, and normal ageing: sample comparisons and normative data. Arch Clin Neuropsychol 2007, 22(4):433-447. dHelmstaedter C, Lendt M, Lux S: Verbaler Lern- und Merkfähigkeitstest (VLMT). Goettingen: Beltz; 2001. e'Lehrl S: Mehrfach-Wortschatz-Intelligenztest MWT-B. Balingen: Spitta Verlag; 1999. ${ }^{\mathrm{f}}$ Zimmermann P, Fimm B: Testbatterie zur Aufmerksamkeitsprüfung (TAP). Version 1.02c. Herzogenrath: PSYTEST; 1993. ${ }^{9}$ Tewes U: Hamburg-Wechsler Intelligenztest fuer Erwachsene (HAWIE-R). Bern: Huber; 1991. hHealthy controls recruited for selected cognitive and olfactory testing (unpublished data).

$32.4 \%$ of variance in the total sample. In fact, duration of disease, negative symptoms, catatonic signs, and medication (chlorpromazine equivalents) contributed significantly to basic cognition/fine motor function, whereas positive symptoms did not $(\beta=-.006, \mathrm{p}=.856)$. According to the standardized regression coefficients, duration of disease and negative symptoms are the best predictors of basic cognition/fine motor function $(\beta=$ $-.346, \mathrm{p}<.001$ and $\beta=-.334, \mathrm{p}<.001)$. For higher cognitive functions, the set of disease-related variables explained $33 \%$ of variance in the total sample. Again, duration of disease and negative symptoms are the best predictors of higher cognitive functions $(\beta=-.335, \mathrm{p}<$ .001 and $\beta=-.351, \mathrm{p}<.001)$. Positive symptoms did not 
Table 4 Self-reported medication side effects of patients $(N=423$ )* according to treatment type

\begin{tabular}{|c|c|c|c|c|}
\hline & \multicolumn{2}{|c|}{$\mathrm{FGA}^{1}$} & \multicolumn{2}{|c|}{$\mathrm{SGA}^{2}$} \\
\hline & men & women & men & women \\
\hline Parkinson symptoms & $17 \%$ & $15.6 \%$ & $3.8 \%$ & $11.6 \%$ \\
\hline dyskinetic/dystonic symptoms & $35.8 \%$ & $31.3 \%$ & $9.4 \%$ & $9.7 \%$ \\
\hline akathisia & $22.6 \%$ & $12.5 \%$ & $6 \%$ & $6.8 \%$ \\
\hline hyperprolactinaemia & - & - & - & $1.9 \%$ \\
\hline hormonal dysfunctions (gynecomastia, absence/changes of menorrhea) & - & $9.4 \%$ & - & $5.8 \%$ \\
\hline sexual dysfunction & $7.5 \%$ & - & $10.3 \%$ & - \\
\hline vertigo (incl. hypotonia) & $5.7 \%$ & $12.5 \%$ & $5.1 \%$ & $8.7 \%$ \\
\hline weight gain & $9.4 \%$ & $18.7 \%$ & $38.3 \%$ & $39.8 \%$ \\
\hline diabetes mellitus & - & - & $0.4 \%$ & - \\
\hline sialorrhea ('drooling') & - & - & $20.4 \%$ & $6.8 \%$ \\
\hline skin abnormalities, loss of hair & $1.9 \%$ & - & $1.7 \%$ & $5.8 \%$ \\
\hline gastrointestinal symptoms & $1.9 \%$ & $6.3 \%$ & $5.9 \%$ & $7.8 \%$ \\
\hline hyperhidrosis & - & - & $2.6 \%$ & - \\
\hline psychological symptoms (loss of concentration, no drive, tiredness) & $33.9 \%$ & $28.1 \%$ & $44.2 \%$ & $31.1 \%$ \\
\hline cardiovascular symptoms (tachycardia, hypertension) & - & - & $1.3 \%$ & $1.9 \%$ \\
\hline impaired vision & - & - & $1.7 \%$ & $3.9 \%$ \\
\hline dry mouth & $5.7 \%$ & $9.4 \%$ & $5.1 \%$ & $4.9 \%$ \\
\hline urinary retention & - & $3.1 \%$ & $1.3 \%$ & - \\
\hline number of patients who reported side effects & 53 & 32 & 235 & 103 \\
\hline
\end{tabular}

reach significance $(\beta=-.015, \mathrm{p}=.658)$. With respect to global functioning, all chosen disease-related factors accounted for $59.6 \%$ of variance in the total sample. Only duration of disease per se did not reach significance ( $\beta=-.028, p=.198)$. Positive and negative symptoms were the strongest predictors of global functioning $(\beta=-.441, \mathrm{p}<.001$ and $\beta=-.380, \mathrm{p}<.001)$.

\section{Discussion}

The present paper provides an overview of the GRAS data collection, including (1) study logistics and procedures, (2) sample description regarding sociodemographic data, disease-related variables, cognitive performance and neurological symptoms, paying particular attention to gender differences, and (3) a first presentation of intercorrelation patterns for selected areas of interest to phenotype studies. (4) In addition, diseaserelated factors influencing important criteria of daily functioning are evaluated in the $>1000$ GRAS patients. Overall, the GRAS sample represents a typical schizophrenic population in contact with the health system and is - last not least due to its homogeneous data acquisition - ideally suited for the ongoing and planned phenotype-based genetic association studies (PGAS) (e.g. [[11], and Grube et al: Calcium-activated potassium channels as regulators of cognitive performance in schizophrenia, submitted]).
The GRAS data collection has several remarkable advantages, two of which are of major importance for its ultimate goal, PGAS: (i) Different from other studies dealing with the establishment of a schizophrenia data base, all data for GRAS were collected by one and the same traveling team of examiners, who frequently performed calibrating sessions and rater trainings. This effort has clearly paid off in terms of reliability and quality of the data, considering the internal consistencies of the GRAS phenotypes, as exemplified in the displayed correlation patterns. (ii) Even though the GRAS study has been implemented as a cross-sectional investigation, the GRAS data collection also includes solid longitudinal information derived from the almost complete psychiatric chart records/discharge letters of all schizophrenic patients. This longitudinal set of data has been essential to e.g. reliably estimate prodrome versus disease onset, i. e. occurrence of the first psychotic episode.

Comparable to other schizophrenia samples, the GRAS sample comprises around two thirds of male and one third of female patients $[17,58]$. Assuming that the gender ratio in schizophrenia were $1: 1$ as claimed in text books, but recently also questioned $[59,60]$, then two principal reasons may account for the gender distribution observed here: (1) Schizophrenic women generally seem to have less contact with the health system due to being better socially settled (later age of onset of 


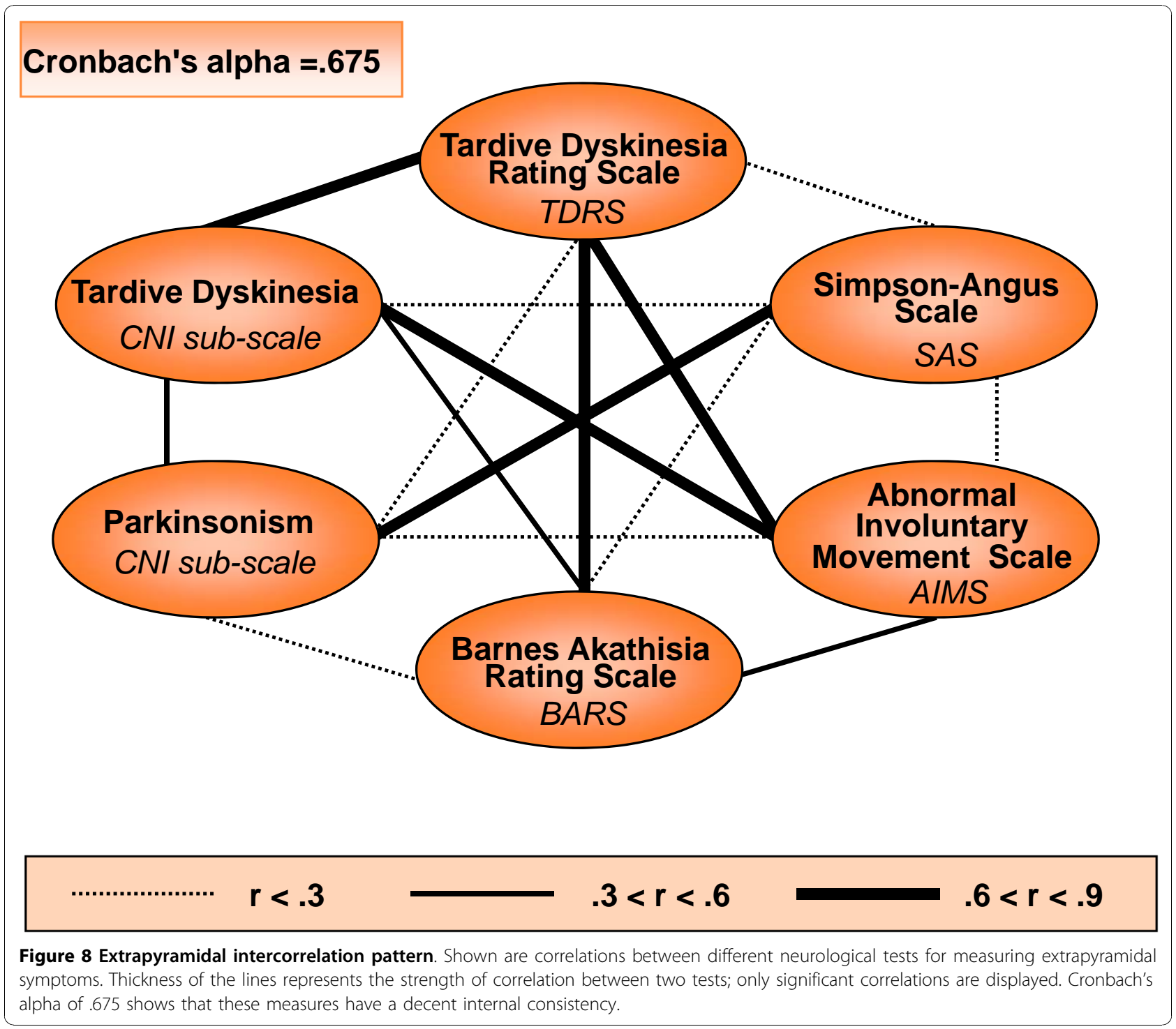

disease) and protected within their families [61]; (2) A certain (smaller) recruitment bias may be explained by the fact that the traveling team of examiners visited some institutions with an overrepresentation of males, e. g. specialized forensic units or a hospital for psychotic patients with co-morbid substance use disorders.

With the purposeful strategy to visit several different facilities of psychiatric health care covering inpatients, outpatients, residents of sheltered homes and forensic patients, the GRAS approach tried to avoid biases inherent to pure inpatient samples [58]. Nevertheless, patients who are not in contact with the health care system are unlikely to be integrated in any comparable data bases. For instance, only 4 of the 1085 examined patients are currently homeless, whereas among homeless people a considerable proportion suffers from schizophrenia [62]. To reach them as well, different and more cost intensive recruitment strategies would be required [13]. On the other hand, the schizophrenic phenotype required for the GRAS-PGAS studies pursued here, might be veiled in this severely affected subsample of patients that is additionally characterized by other specific problems, e. g. a highly elevated incidence of multiple substance use disorders and severe downstream medical comorbidities $[63,64]$.

Gender differences in schizophrenia as obvious from the present data collection have been known for a long time [65]. In agreement with the literature, men and women in the GRAS sample differ by diagnosis, with women having a higher rate of schizoaffective disorders $[66,67]$. With respect to age of onset, education, indicators of social integration (e.g. marital status, living situation) and medication, the present results are also in perfect agreement with previous findings: Male patients 
Table 5 Cambridge Neurological Inventory (CNI)a subscale sum scores ( $\mathbf{N}=\mathbf{8 9 3 - 9 4 2 )}$

\begin{tabular}{|c|c|c|c|c|c|c|c|c|}
\hline \multirow[b]{2}{*}{ sub scales } & \multicolumn{2}{|c|}{ total } & \multicolumn{2}{|c|}{ men } & \multicolumn{2}{|c|}{ women } & \multicolumn{2}{|c|}{ statistics } \\
\hline & $\begin{array}{c}\text { Mean } \\
(\text { sd) }\end{array}$ & $\begin{array}{l}\text { Median } \\
\text { (range) }\end{array}$ & $\begin{array}{c}\text { Mean } \\
(s d)\end{array}$ & $\begin{array}{l}\text { Median } \\
\text { (range) }\end{array}$ & $\begin{array}{c}\text { Mean } \\
(\text { sd) }\end{array}$ & $\begin{array}{l}\text { Median } \\
\text { (range) }\end{array}$ & $\mathbf{Z}$ & $\mathbf{p}$ \\
\hline \multicolumn{9}{|l|}{ Hard neurological signs } \\
\hline $\begin{array}{l}\text { plantar reflexes (le/ri*), power in upper and lower limb (le/ri), and reflexes } \\
\text { (hyper- and hyporeflexia) in upper and lower limb (le/ri) }\end{array}$ & $\begin{array}{c}1.12 \\
(1.70)\end{array}$ & $\begin{array}{l}0.0(0- \\
10)\end{array}$ & $\begin{array}{l}1.07 \\
(1.66)\end{array}$ & $0.0(0-8)$ & $\begin{array}{c}1.22 \\
(1.78)\end{array}$ & $\begin{array}{l}0.0(0- \\
10)\end{array}$ & -1.467 & n.s \\
\hline \multicolumn{9}{|l|}{ Motor coordination } \\
\hline $\begin{array}{l}\text { finger-nose test (le/ri), finger-thumb tapping (le/ri), finger-thumb opposition } \\
\text { (le/ri), pronation-supination (le/ri); fist-edge-palm test (le/ri), Oseretsky test }\end{array}$ & $\begin{array}{l}4.11 \\
(4.27)\end{array}$ & $\begin{array}{l}3.0(0- \\
20)\end{array}$ & $\begin{array}{l}3.95 \\
(4.17)\end{array}$ & $\begin{array}{l}2.0(0- \\
20)\end{array}$ & $\begin{array}{c}4.44 \\
(4.45)\end{array}$ & $\begin{array}{l}3.0(0- \\
20)\end{array}$ & -1.629 & n.s \\
\hline \multicolumn{9}{|l|}{ Sensory integration } \\
\hline $\begin{array}{l}\text { extinction, finger agnosia (le/ri), stereoagnosia (le/ri), agraphesthesia (le/ri), } \\
\text { left-right disorientation }\end{array}$ & $\begin{array}{c}3.66 \\
(3.32)\end{array}$ & $\begin{array}{l}3.0(0- \\
15)\end{array}$ & $\begin{array}{c}3.63 \\
(3.32)\end{array}$ & $\begin{array}{l}3.0(0- \\
15)\end{array}$ & $\begin{array}{l}3.73 \\
(3.31)\end{array}$ & $\begin{array}{l}3.0(0- \\
14)\end{array}$ & -0.521 & n.s \\
\hline \multicolumn{9}{|l|}{ Primitive reflexes } \\
\hline snout reflex, grasp reflex, palmo-mental reflex (le/ri) & $\begin{array}{c}0.84 \\
(1.14)\end{array}$ & $0.0(0-5)$ & $\begin{array}{c}0.80 \\
(1.11)\end{array}$ & $0.0(0-5)$ & $\begin{array}{c}0.91 \\
(1.19)\end{array}$ & $0.0(0-5)$ & -1.363 & n.s \\
\hline \multicolumn{9}{|l|}{ Tardive dyskinesia } \\
\hline $\begin{array}{l}\text { dyskinetic, sustained or manneristic face and head movement, simple or } \\
\text { complex abnormal posture, dyskinetic, dystonic or manneristic trunk/limb } \\
\text { movement }\end{array}$ & $\begin{array}{c}0.55 \\
(1.17)\end{array}$ & $0.0(0-9)$ & $\begin{array}{c}0.58 \\
(1.25)\end{array}$ & $0.0(0-9)$ & $\begin{array}{c}0.49 \\
(0.98)\end{array}$ & $0.0(0-7)$ & -0.132 & n.s \\
\hline \multicolumn{9}{|l|}{ Catatonic signs } \\
\hline $\begin{array}{l}\text { gait mannerism, gegenhalten, mitgehen, imposed posture, exaggerated or } \\
\text { iterative movement, automatic obedience, echopraxia }\end{array}$ & $\begin{array}{l}0.43 \\
(0.96)\end{array}$ & $0.0(0-8)$ & $\begin{array}{l}0.45 \\
(0.98)\end{array}$ & $0.0(0-8)$ & $\begin{array}{l}0.38 \\
(0.91)\end{array}$ & $0.0(0-7)$ & -1.717 & n.s \\
\hline \multicolumn{9}{|l|}{ Parkinsonism } \\
\hline $\begin{array}{l}\text { increased tone in upper and lower limb (le/ri), decreased associated } \\
\text { movements in walking, shuffling gait, arm dropping, tremor postural or } \\
\text { resting, rigidity in neck }\end{array}$ & $\begin{array}{l}1.76 \\
(2.90)\end{array}$ & $\begin{array}{l}0.0(0- \\
15)\end{array}$ & $\begin{array}{l}1.70 \\
(2.85)\end{array}$ & $\begin{array}{l}0.0(0- \\
15)\end{array}$ & $\begin{array}{c}1.89 \\
(3.02)\end{array}$ & $\begin{array}{l}0.5(0- \\
15)\end{array}$ & -1.172 & n.s \\
\hline \multicolumn{9}{|l|}{ Failure to suppress inappropriate response } \\
\hline $\begin{array}{l}\text { blinking or head movement in saccadic eye movement, winking with one } \\
\text { eye }\end{array}$ & $\begin{array}{c}1.23 \\
(1.49)\end{array}$ & $1.0(0-6)$ & $\begin{array}{c}1.12 \\
(1.42)\end{array}$ & $1.0(0-6)$ & $\begin{array}{c}1.48 \\
(1.62)\end{array}$ & $1.0(0-6)$ & -3.175 & $.001^{*}$ \\
\hline
\end{tabular}

*le/ri - left and right

${ }^{a}$ Chen EY, Shapleske J, Luque R, McKenna PJ, Hodges JR, Calloway SP, Hymas NF, Dening TR, Berrios GE: The Cambridge Neurological Inventory: a clinical instrument for assessment of soft neurological signs in psychiatric patients. Psychiatry Res 1995, 56(2):183-204.

are younger when the first psychotic episode occurs, are more frequently single, more often dependent on supported living conditions (e.g. residential homes) and show lower educational status $[61,67,68]$. Among the explanations for the observed gender differences in schizophrenia are the protective role of female hormones [69] and social aspects like earlier marriage of young women leading to a more protected environment at disease onset [13]. In line with these considerations is the work of Häfner and colleagues [12]. In a prospective design he could show that 'the social course (of schizophrenia) is determined by individual stage at illness onset and by early illness course' [70].

With respect to psychopathology and premorbid functioning, the GRAS sample may be slightly different from other schizophrenia samples reported in the literature [67]. Several studies published in this area show that men exhibit more negative symptoms, even in a geriatric sample [71,72], and that females have poorer premorbid cognitive functioning than males [73]. In the GRAS patients, there are no gender differences regarding psychopathology and premorbid cognition. Importantly, clear support for a comparable severity of psychopathology in men and women of the GRAS sample is provided by the lack of gender differences in numbers of hospitalizations, clinical severity ratings, including global functioning (CGI, GAF $[2,31])$, and self-ratings of symptom severity and anxiety. One potential explanation for the discrepancies between the GRAS sample and other studies regarding psychopathology may be that patient numbers in some of the other studies have been too low to give conclusive results. In the assessment of premorbid cognitive functioning of the GRAS sample, a methodological limitation could be the retrospective determination of premorbid intelligence using a so-called 'hold' measure, i.e. a multiple choice vocabulary test [35]. Even though this is an accepted and valid instrument to retrospectively estimate premorbid intelligence [74], a prospective procedure might be more accurate. In fact, Weiser and colleagues had the opportunity to base their assessments on cognitive testing performed on adolescents before starting their military service [73], potentially explaining the deviating results.

Gender differences regarding current cognitive performance are similar within the GRAS sample (even though 
Table 6 Multiple regression analyses predicting a) basic cognition/fine motor functions, b) cognitive performance, c) global functioning

\begin{tabular}{|c|c|c|c|c|c|c|c|c|c|}
\hline & \multicolumn{3}{|c|}{ total } & \multicolumn{3}{|c|}{ male } & \multicolumn{3}{|c|}{ female } \\
\hline & $\beta$ & $\mathbf{t}$ & $\mathrm{p}$ & $\beta$ & $\mathrm{t}$ & $\mathbf{p}$ & $\beta$ & $\mathbf{t}$ & $\mathbf{p}$ \\
\hline \multicolumn{10}{|c|}{ a) basic cognition/fine motor functions ${ }^{1}$} \\
\hline duration of disease (years) & -.346 & -11.92 & $<.001$ & -.353 & -9.68 & $<.001$ & -.318 & -6.59 & $<.001$ \\
\hline positive symptoms (PANSS) & -.006 & -0.18 & .856 & -.028 & -0.69 & .489 & .065 & 1.08 & .283 \\
\hline negative symptoms (PANSS) & -.334 & -10.05 & $<.001$ & -.293 & -7.32 & $<.001$ & -.415 & -7.01 & $<.001$ \\
\hline catatonic signs $(C N)$ & -.126 & -4.26 & $<.001$ & -.128 & -3.45 & .001 & -.161 & -3.27 & .001 \\
\hline medication (CPZ-equivalents) & -.080 & -2.70 & .007 & -.066 & -1.83 & .068 & -.147 & -2.84 & .005 \\
\hline regression model & & $\begin{array}{l}r^{2}=.324 \\
p<.001\end{array}$ & & & $\begin{array}{l}r^{2}=.306 \\
p<.001\end{array}$ & & & $\begin{array}{l}r^{2}=.383 \\
p<.001\end{array}$ & \\
\hline \multicolumn{10}{|l|}{ b) cognitive performance ${ }^{2}$} \\
\hline duration of disease (years) & -.335 & -11.54 & $<.001$ & -.356 & -9.72 & $<.001$ & -.294 & -6.12 & $<.001$ \\
\hline positive symptoms (PANSS) & -.015 & -0.44 & .658 & -.033 & -0.80 & .427 & .023 & 0.38 & .704 \\
\hline negative symptoms (PANSS) & -.351 & -10.47 & $<.001$ & -.320 & -7.92 & $<.001$ & -.396 & -6.56 & $<.001$ \\
\hline catatonic signs (CNI) & -.132 & -4.46 & $<.001$ & -.103 & -2.76 & .006 & -.204 & -4.16 & $<.001$ \\
\hline medication (CPZ-equivalents) & -.082 & -2.74 & .006 & -.060 & -1.62 & .105 & -.140 & -2.70 & .007 \\
\hline regression model & & $\begin{array}{l}r^{2}=.330 \\
p<.001\end{array}$ & & & $\begin{array}{l}r^{2}=.305 \\
p<.001\end{array}$ & & & $\begin{array}{l}r^{2}=.394 \\
p<.001\end{array}$ & \\
\hline \multicolumn{10}{|l|}{ c) global functioning ${ }^{3}$} \\
\hline duration of disease (years) & -.028 & -1.29 & .198 & -.008 & -0.28 & .780 & -.062 & -1.78 & .076 \\
\hline positive symptoms (PANSS) & -.441 & -17.33 & $<.001$ & -.458 & -14.45 & $<.001$ & -.415 & -9.60 & $<.001$ \\
\hline negative symptoms (PANSS) & -.380 & -15.02 & $<.001$ & -.345 & -10.97 & $<.001$ & -.430 & -10.0 & $<.001$ \\
\hline catatonic signs $(C N)$ & -.060 & -2.67 & .008 & -.050 & -1.71 & .088 & -.093 & -2.58 & .011 \\
\hline medication (CPZ-equivalents) & -.106 & -4.71 & $<.001$ & -.122 & -4.29 & $<.001$ & -.078 & -2.07 & .040 \\
\hline regression model & & $\begin{array}{l}r^{2}=.596 \\
p<.001\end{array}$ & & & $\begin{array}{l}r^{2}=.559 \\
p<.001\end{array}$ & & & $\begin{array}{l}r^{2}=.662 \\
p<.001\end{array}$ & \\
\hline
\end{tabular}

${ }^{1} \mathrm{~A}$ basic cognition/fine motor composite score was used as a dependent variable comprising alertness (TAP), tapping, and dotting (Chronbachs alpha $=.801$ ). ${ }^{2} \mathrm{~A}$ cognitive composite score was used as a dependent variable consisting of reasoning (LPS3), 2 processing speed measures (TMT -A and digit-symbol test, ZST), executive functions (TMT-B), working memory (BZT), verbal memory (VLMT) and divided attention (TAP) (Chronbachs alpha $=.869$ ).

${ }^{3} \mathrm{Global}$ assessment of functioning (GAF) was used as a dependent variable.

at a lower functioning level [75]) compared to healthy controls [76] after considering age of onset, duration of disease, education and medication as covariates. Men perform better in reasoning, alertness and divided attention but worse in verbal memory, confirming reports on first-episode as well as chronically ill schizophrenic patients [77].

Women in the GRAS study receive significantly lower doses of chlorpromazine equivalents, confirming that they require less medication to achieve a reasonable treatment effect [78]. Importantly, regarding medication side effects, there were no gender differences in extrapyramidal symptoms. There were also no differences in the overall proportion of men and women who selfreported side effects, but the pattern of complaints was slightly different. For instance, women mentioned more often hormonal dysfunction and vertigo (or related symptoms like hypotonia), whilst men complained mainly about sexual dysfunction. Altogether, it is worth pointing out that the percentage of patients self-reporting side effects is low when compared to that with objectively measured side effects, e.g. extrapyramidal symptoms (11.3\% versus $32.3 \%)$.

Explicit studies on gender differences in antipsychotic medication side effects found a somewhat different distribution of complaints, e.g. more weight gain, diabetes and specific cardiovascular diseases in females [78,79]. Here, one reason is certainly the still preliminary data set of the GRAS collection evaluated, based at this point exclusively on cross-sectional patient reports. For a more appropriate coverage of medication side effects, all charts/discharge letters of every GRAS patient (also of those patients who did/could not report them), will have to be screened and entered into the data base. Comprehensive information on antipsychotic (and other) drugs and their side effects in the GRAS sample has been collected and is waiting for analyses to support e.g. future pharmacogenomic approaches, perhaps also in collaboration with industry partners.

In line with the findings of a recent meta-analysis [80], positive symptoms of the GRAS patients do not influence higher cognitive function or basic cognition/fine 
motor performance, whilst negative symptoms, catatonic signs, duration of disease and antipsychotic medication have a significant effect on both. The clinical ratings of global functioning, however, strongly rely on positive as well as negative symptoms, medication and catatonic signs [81-83].

\section{Conclusion}

GRAS enables a novel phenotype-based approach to understand the molecular-genetic architecture of schizophrenia. The GRAS data collection encompasses a large sample of comprehensively phenotyped, moderately to severely affected schizophrenic patients. Proof-of-principle for the suitability of the GRAS data collection for PGAS has already been demonstrated [[11], and Grube et al: Calcium-activated potassium channels as regulators of cognitive performance in schizophrenia, submitted]. Further extensive analyses of the accumulated information on every single patient are ongoing.

\section{Abbreviations}

GRAS: Göttingen Research Association for Schizophrenia; GWAS: genomewide association study; PGAS: phenotype-based genetic association study; CATIE: clinical antipsychotic trials of intervention effectiveness; CNI: Cambridge Neurological Inventory; ASRB: Australian Schizophrenia Research Bank; FGA: first generation antipsychotics; SGA: second generation antipsychotics; CPZ: chlorpromazine.

\section{Acknowledgements}

This study was supported by the Max Planck Society and the DFG-Research Center for Molecular Physiology of the Brain (CMBP). We are indebted to all patients for their participation in the GRAS (Göttingen Research Association for Schizophrenia) study and to all colleagues in the collaborating centers who contributed to the GRAS data collection.

\section{Author details}

${ }^{1}$ Division of Clinical Neuroscience, Max Planck Institute of Experimental Medicine, Göttingen, Germany. ${ }^{2}$ Department of Psychiatry and Psychotherapy, Ecumenical Hospital Hainich, Germany. ${ }^{3}$ Hospital of Psychiatry and Psychotherapy, Center for Integrative Psychiatry, Kiel, Germany. ${ }^{4}$ Karl-Jaspers-Hospital, Psychiatric Federation Oldenburger Land, Bad Zwischenahn, Germany. ${ }^{5}$ Department of Psychiatry II, UIm University, District Hospital Günzburg, Germany. ${ }^{6}$ Department of Psychiatry and Psychotherapy, Hospital Fulda, Germany. ${ }^{7}$ Department of Psychiatry and Psychotherapy, Isar-Amper-Hospital, Taufkirchen (Vils), Germany. ${ }^{8}$ Department of Psychiatry and Psychotherapy, Reinhard-Nieter Hospital, Wilhelmshaven, Germany. 'Vitos Hospital of Forensic Psychiatry Eltville, Eltville, Germany. ${ }^{10}$ Vitos Hospital of Psychiatry and Psychotherapy Merxhausen, Kassel, Germany. " Department of Psychiatry and Psychotherapy, University of Rostock, Germany. ${ }^{12} \mathrm{Hospital}$ of Forensic Psychiatry, Moringen, Germany. ${ }^{13}$ Hospital of Psychiatry and Psychotherapy Langenhagen, Regional Hospitals Hannover, Germany. ${ }^{14}$ Vitos Hospital of Psychiatry and Psychotherapy, Bad Emstal-Merxhausen, Germany. ${ }^{15}$ Addiction Hospital "Am Waldsee", Rieden, Germany. ${ }^{16}$ Department of Psychiatry and Psychotherapy, University Medical Center of Bonn, Germany. ${ }^{17}$ Vitos Hospital of Psychiatry and Psychotherapy Merxhausen, Hofgeismar, Germany. ${ }^{18}$ Vitos Haina Forensic Psychiatric Hospital, Haina, Germany. ${ }^{19}$ Department of Psychiatry and Psychotherapy, Regional Hospitals Hannover, Wunstorf, Germany. ${ }^{20}$ Dr. K. Fontheim's Hospital for Mental Health, Liebenburg, Germany. ${ }^{21}$ Department of Psychiatry and Psychotherapy, Hospital Ingolstadt, Germany. ${ }^{22}$ Department of Psychiatry and Psychotherapy, Hospital Lübbecke, Germany. ${ }^{23}$ Hospital of Psychiatry and Psychotherapy, Rickling, Germany. ${ }^{24}$ Georg-Elias-Müller-Institute for Psychology, University of Göttingen, Germany. ${ }^{25}$ Department of Psychiatry and Psychotherapy, University Medical Center of Göttingen, Germany.
${ }^{26}$ Biomedical NMR Research GmbH, Max Planck Institute of Biophysical Chemistry, Göttingen, Germany. ${ }^{27}$ Department of Molecular Biology of Neuronal Signals, Max Planck Institute of Experimental Medicine, Göttingen, Germany. ${ }^{28}$ Department of Molecular Neurobiology, Max Planck Institute of Experimental Medicine, Göttingen, Germany. ${ }^{29}$ Department of Neurogenetics, Max Planck Institute of Experimental Medicine, Göttingen, Germany. ${ }^{30}$ DFG Research Center for Molecular Physiology of the Brain (CMPB), Germany.

${ }^{31}$ Founders of the GRAS Initiative.

\section{Authors' contributions}

$\mathrm{MB}$ coordinated and supervised the traveling team of investigators and had a considerable impact on design and establishment of the data collection. $\mathrm{KR}$ and HFr were part of the traveling team of investigators, conducted statistical analyses of the clinical data, assisted in manuscript writing, and supervised data entry, substantially performed by SG, SP, AK, MFG, VA, ATa, ATr, and MF. Of the collaborating centers, $L A, J B A, M B E, T B, A C, M D, H F o, R F$, RG, SH, DH, GK, HK, MFr, FL, WM, AM, RMI, CO, FGP, TP, US, HJS and UHR enabled the work of the traveling team of examiners, by pre-selecting and preparing patients and organizing respective facilities and working conditions. HE, KAN, NB, PF, WS, and JF developed the concept of GRAS (Göttingen Research Association for Schizophrenia, founded in 2004), and guided the project, data analysis, and paper writing, hereby supported by $\mathrm{BKH}$. All authors discussed the results, commented on the paper draft and approved the final version of the manuscript.

\section{Competing interests}

The authors declare that they have no competing interests.

Received: 24 August 2010 Accepted: 10 November 2010 Published: 10 November 2010

\section{References}

1. Jablensky A, Sartorius N, Ernberg G, Anker M, Korten A, Cooper JE, Day R, Bertelsen A: Schizophrenia: manifestations, incidence and course in different cultures. A World Health Organization ten-country study. Psychol Med Monogr Suppl 1992, 20:1-97.

2. American Psychiatric Association: Diagnostic and statistical manual of mental disorders, (DSM-IV). 4 edition. Washington, DC: American Psychiatric Press; 1994.

3. World Health Organization: The ICD-10 classification of mental and behavioural disorders: Clinical descriptions and diagnostic guidelines Geneva: WHO; 1992

4. Cardno AG, Gottesman II: Twin studies of schizophrenia: from bow-andarrow concordances to starwars $\mathrm{Mx}$ and functional genomics. Am J Med Genet 2000, 97(1):12-17.

5. Lichtenstein P, Yip BH, Björk C, Pawitan Y, Cannon TD, Sullivan PF, Hultman CM: Common genetic determinants of schizophrenia and bipolar disorder in Swedish families: a population-based study. The Lancet 2009, 373(9659):234-239.

6. Franzek E, Beckmann H: Different Genetic Background of Schizophrenia Spectrum Psychoses: A Twin Study. Am J Psychiatry 1998, 155(1):76-83.

7. Cardno AG, Marshall EJ, Coid B, Macdonald AM, Ribchester TR, Davies NJ, Venturi P, Jones LA, Lewis SW, Sham PC, Gottesman II, Farmer AE, McGuffin P, Reveley AM, Murray RM: Heritability Estimates for Psychotic Disorders: The Maudsley Twin Psychosis Series. Arch Gen Psychiatry 1999, 56(2):162-168.

8. Gottesman I I, Shields J: A polygenic theory of schizophrenia. Proc Natl Acad Sci USA 1967, 58(1):199-205.

9. Riley B, Asherson PJ, McGuffin P: Genetics and schizophrenia. In Schizophrenia. Edited by: Hirsch SR, Weinberger DR. Massachusetts: Blackwell Publishing; 2003:

10. Duan J, Sanders AR, Gejman PV: Genome-wide approaches to schizophrenia. Brain Research Bulletin

11. Begemann M, Grube S, Papiol S, Malzahn D, Krampe H, Ribbe K, Friedrichs H, Radyushkin KA, El-Kordi A, Benseler F, Hannke K, Sperling S, Schwerdtfeger D, Thanhauser I, Gerchen MF, Ghorbani M, Gutwinski S, Hilmes C, Leppert R, Ronnenberg A, Sowislo J, Stawicki S, Stodtke M, Szuszies C, Reim K, Riggert J, Eckstein F, Falkai P, Bickeboller H, Nave KA, et al: Modification of cognitive performance in schizophrenia by complexin 2 gene polymorphisms. Arch Gen Psychiatry 2010, 67(9):879-888. 
12. Häfner $H$, Maurer $K$, Löffler W, an der Heiden W, Munk-Jørgensen $P$, Hambrecht M, Riecher-Rössler A: The ABC schizophrenia study: a preliminary overview of the results. Social Psychiatry and Psychiatric Epidemiology 1998, 33(8):380-386.

13. Jablensky A, McGrath J, Herrman H, Castle D, Gureje O, Evans M, Carr V, Morgan V, Korten A, Harvey C: Psychotic disorders in urban areas: an overview of the Study on Low Prevalence Disorders. Aust N Z J Psychiatry 2000, 34(2):221-236.

14. Haro JM, Edgell ET, Jones PB, Alonso J, Gavart S, Gregor KJ, Wright P, Knapp M: The European Schizophrenia Outpatient Health Outcomes (SOHO) study: rationale, methods and recruitment. Acta Psychiatr Scand 2003, 107(3):222-232.

15. Karayiorgou M, Torrington M, Abecasis GR, Pretorius $H$, Robertson $B$, Kaliski S, Lay S, Sobin C, Möller N, Lundy SL, Blundell ML, Gogos JA, Roos JL: Phenotypic characterization and genealogical tracing in an Afrikaner schizophrenia database. American Journal of Medical Genetics Part B: Neuropsychiatric Genetics 2004, 124B(1):20-28.

16. Fleischhacker WW, Keet IP, Kahn RS: The European First Episode Schizophrenia Trial (EUFEST): rationale and de sign of the trial. Schizophr Res 2005, 78(2-3):147-156.

17. Lieberman JA, Stroup TS, McEvoy JP, Swartz MS, Rosenheck RA, Perkins DO, Keefe RS, Davis SM, Davis CE, Lebowitz BD, Severe J, Hsiao JK: Effectiveness of antipsychotic drugs in patients with chronic schizophrenia. N Engl J Med 2005, 353(12):1209-1223

18. Price GW, Michie PT, Johnston J, Innes-Brown H, Kent A, Clissa P, Jablensky AV: A Multivariate Electrophy siological Endophenotype, from a Unitary Cohort, Shows Greater Research Utility than Any Single Feature in the Western Australian Family Study of Schizophrenia. Biological Psychiatry 2006, 60(1):1-10.

19. Calkins ME, Dobie DJ, Cadenhead KS, Olincy A, Freedman R, Green MF, Greenwood TA, Gur RE, Gur RC, Light GA, Mintz J, Nuechterlein KH, Radant AD, Schork NJ, Seidman LJ, Siever LJ, Silverman JM, Stone WS, Swerdlow NR, Tsuang DW, Tsuang MT, Turetsky BI, Braff DL: The Consortium on the Genetics of Endophenotypes in Schizophrenia: Model Recruitment, Assessment, and Endophenotyping Methods for a Multisite Collaboration. Schizophr Bull 2007, 33(1):33-48.

20. Allen NC, Bagade S, McQueen MB, loannidis JPA, Kavvoura FK, Khoury MJ, Tanzi RE, Bertram L: Systematic meta-analy ses and field synopsis of genetic association studies in schizophrenia: the SzGene database. Nat Genet 2008, 40(7):827-834.

21. Stroup TS, McEvoy JP, Swartz MS, Byerly MJ, Glick ID, Canive JM, McGee MF, Simpson GM, Stevens MC, Lieberman JA: The National Institute of Mental Health Clinical Antipsychotic Trials of Intervention Effectiveness (CATIE) project: schizophrenia trial design and protocol development. Schizophr Bull 2003, 29(1):15-31.

22. Poyastro Pinheiro A, Keefe RSE, Skelly T, Olarte M, Leviel $K$, Lange LA, Lange EM, Scott Stroup T, Lieberman J, Sullivan PF: AKT1 and neurocognition in schizophrenia. Australian and New Zealand Journal of Psychiatry 2009, 41(2):169-177.

23. Sullivan PF, Keefe RSE, Lange LA, Lange EM, Stroup TS, Lieberman J, Maness PF: NCAM1 and Neurocognition in Schizophrenia. Biological Psychiatry 2007, 61(7):902-910.

24. Crowley JJ, Keefe RSE, Perkins DO, Stroup TS, Lieberman JA, Sullivan PF: The neuregulin 1 promoter polymorphism rs6994992 is not associated with chronic schizophrenia or neurocognition. American Journal of Medical Genetics Part B: Neuropsychiatric Genetics 2008, 147B(7):1298-1300.

25. Sullivan PF, Lin D, Tzeng JY, van den Oord E, Perkins D, Stroup TS, Wagner M, Lee S, Wright FA, Zou F, Liu W, Downing AM, Lieberman J, Close SL: Genomewide association for schizophrenia in the CATIE study: results of stage 1. Mol Psychiatry 2008, 13(6):570-584.

26. Australian Schizophrenia Research Bank. [http://www schizophreniaresearch.org.au/bank/index.php].

27. Loughland CM, Carr VJ, Lewin TJ: The NISAD Schizophrenia Research Register: why do we need a database of schizophrenia volunteers? Aust N Z J Psychiatry 2001, 35(5):660-667.

28. Loughland C, Draganic D, McCabe K, Richards J, Nasir A, Allen J, Catts S, Jablensky A, Henskens F, Michie P, Mowry B, Pantelis C, Schall U, Rodney S, Tooney P, Carr V: The Australian Schizophrenia Research Bank: A database of comprehensive clinical, endophenotypic and genetic data for etiological studies of schizophrenia. Australian and New Zealand Journal of Psychiatry.
29. Wittchen H-U, Zaudig M, Fydrich T: SKID-I (Strukturiertes Klinisches Interview für DSM-IV; Achse I: Psychische Störungen) Göttingen: Hogrefe; 1997.

30. Kay SR, Fiszbein A, Opler LA: The positive and negative syndrome scale (PANSS) for schizophrenia. Schizophr Bull 1987, 13(2):261-276.

31. Guy W: Clinical Global Impression (CGI). ECDEU Assessment manual for psychopharmacology, revised National Institue of Mental Health. Rockville, MD 1976.

32. Laux L, Glanzmann P, Schaffner P, Spielberger CD: Das State-TraitAngstinventar (STAI) Weinheim: Beltz; 1981

33. Franke GH: Brief Symptom Inventory (BSI) Goettingen: Beltz; 2000.

34. Kupfer J, Brosig B, Braehler E: Toronto Alexithymie-Skala-26 (TAS-26) Goettingen: Hogrefe; 2001.

35. Lehrl S, Triebig G, Fischer B: Multiple choice vocabulary test MWT as a valid and short test to estimate premorbid intelligence. Acta Neurol scand 1995, 91(5):335-345.

36. Lehrl S: Mehrfach-Wortschatz-Intelligenztest MWT-B Balingen: Spitta Verlag; 1999.

37. Horn W: Leistungsprüfsystem (LPS). 2 edition. Goettingen: Hogrefe; 1983.

38. Gold JM, Carpenter C, Randolph C, Goldberg TE, Weinberger DR: Auditory working memory and Wisconsin Card Sorting Test performance in schizophrenia. Arch Gen Psychiatry 1997, 54(2):159-165.

39. Chapman RL: The MacQuarrie test for mechanical ability. Psychometrika 1948, 13(3):175-179.

40. War-Department: Army Individual Test Battery. Manual of directions and scoring Washington, D.C.: War Department, Adjutant General's Office; 1944.

41. Perianez JA, Rios-Lago M, Rodriguez-Sanchez JM, Adrover-Roig D, SanchezCubillo I, Crespo-Facorro B, Quemada Jl, Barcelo F: Trail Making Test in traumatic brain injury, schizophrenia, and normal ageing: sample comparisons and normative data. Arch Clin Neuropsychol 2007, 22(4):433-447.

42. Kessler J, Denzler P, Markowitsch HJ: Demenz-Test (DT) Göttingen: Hogrefe; 1999.

43. Aschenbrenner S, Tucha O, Lange KW: Der Regensburger Wortflüssigkeits-Test (RWT) Göttingen: Hogrefe; 2000.

44. Tewes U: Hamburg-Wechsler Intelligenztest fuer Erwachsene (HAWIE-R) Bern: Huber; 1991.

45. Helmstaedter C, Lendt M, Lux S: Verbaler Lern- und Merkfähigkeitstest (VLMT) Goettingen: Beltz; 2001

46. Zimmermann P, Fimm B: Testbatterie zur Aufmerksamkeitsprüfung (TAP) Version 1.02C Herzogenrath: PSYTEST; 1993.

47. Chen EY, Shapleske J, Luque R, McKenna PJ, Hodges JR, Calloway SP, Hymas NF, Dening TR, Berrios GE: The Cambridge Neurological Inventory: a clinical instrument for assessment of soft neurological signs in psychiatric patients. Psychiatry Res 1995, 56(2):183-204.

48. Bartels C, Mertens N, Hofer S, Merboldt KD, Dietrich J, Frahm J, Ehrenreich $\mathrm{H}$ : Callosal dysfunction in amyotrophic lateral sclerosis correlates with diffusion tensor imaging of the central motor system. Neuromuscul Disord 2008, 18(5):398-407.

49. Barnes TR: The Barnes Akathisia Rating Scale - revisited. J Psychopharmacol 2003, 17(4):365-370.

50. Simpson GM, Angus JW: A rating scale for extrapyramidal side effects. Acta Psychiatr Scand Suppl 1970, 212:11-19.

51. Simpson GM, Lee $J H$, Zoubok B, Gardos G: A rating scale for tardive dyskinesia. Psychopharmacology (Berl) 1979, 64(2):171-179.

52. Guy W: Abnormal involuntary movement scale (AIMS). ECDEU Assessment manual for psychopharmacology, revised National Institute of Mental Health. Rockville, MD 1976.

53. Krampe H, Bartels C, Victorson D, Enders CK, Beaumont J, Cella D, Ehrenreich $\mathrm{H}$ : The influence of personality factors on disease progression and health-related quality of life in people with ALS. Amyotroph Lateral Scler 2008, 9(2):99-107.

54. SPSS Inc. [http://www.spss.com/].

55. Blom G: Statistical Estimates and Transformed Beta Variables New York: John Wiley and Sons, Inc; 1958.

56. Davis JM: Comparative doses and costs of antipsychotic medication. Arch Gen Psychiatry 1976, 33(7):858-861.

57. Barnes TR: A rating scale for drug-induced akathisia. Br J Psychiatry 1989 $154: 672-676$

58. Loughland CM, Carr VJ, Lewin TJ, Barnard RE, Chapman JL, Walton JM: Potential sampling and recruitment source impacts in schizophrenia research. Psychiatry Res 2004, 125(2):117-127. 
59. Aleman A, Kahn RS, Selten J-P: Sex differences in the risk of schizophrenia: evidence from meta-analysis. Arch Gen Psychiatry 2003, 60(6):565-571.

60. McGrath J, Saha S, Chant D, Welham J: Schizophrenia: a concise overview of incidence, prevalence, and mortality. Epidemiol Rev 2008, 30:67-76.

61. Riecher-Rossler A, Hafner $\mathrm{H}$ : Gender aspects in schizophrenia: bridging the border between social and biological psychiatry. Acta Psychiatr Scand Suppl 2000, , 407: 58-62.

62. Henry J-M, Boyer L, Belzeaux R, Baumstarck-Barrau K, Samuelian J-C: Mental Disorders Among Homeless People Admitted to a French Psychiatric Emergency Service. Psychiatr Serv 2010, 61(3):264-271.

63. Toro PA, Bellavia CW, Daeschler CV, Owens BJ, Wall DD, Passero JM, Thomas DM: Distinguishing homelessness from poverty: A comparative study. Journal of Consulting and Clinical Psychology 1995, 63(2):280-289.

64. Israel N, Toro P, Ouellette N: Changes in the Composition of the Homeless Population: 1992-2002. American Journal of Community Psychology 2010, 46(1-2):49-59.

65. Kraepelin E: Psychiatrie. Ein Lehrbuch für Studierende und Ärzte. Sechste, vollständig umgearbeitete Auflage Leipzig: Johann Ambrosius Barth; 1899.

66. Castle DJ, Sham PC, Wessely S, Murray RM: The subtyping of schizophrenia in men and women: a latent class analysis. Psychol Med 1994, 24(1):41-51.

67. Leung $A$, Chue P: Sex differences in schizophrenia, a review of the literature. Acta Psychiatr Scand Suppl 2000, 401:3-38.

68. Larsen TK, McGlashan TH, Johannessen JO, Vibe-Hansen L: First-episode schizophrenia: II. Premorbid patterns by gender. Schizophr Bull 1996, 22(2):257-269.

69. Seeman MV, Lang M: The role of estrogens in schizophrenia gender differences. Schizophr Bull 1990, 16(2):185-194.

70. Häfner H: Onset and early course as determinants of the further course of schizophrenia. Acta Psychiatr Scand Suppl 2000, , 407: 44-48.

71. Moriarty PJ, Lieber D, Bennett A, White L, Parrella M, Harvey PD, Davis KL: Gender differences in poor outcome patients with lifelong schizophrenia. Schizophr Bull 2001, 27(1):103-113.

72. Thorup A, Petersen $L$, Jeppesen P, Ohlenschlaeger J, Christensen $T$, Krarup $G$, Jorgensen $P$, Nordentoft M: Gender differences in young adults with first-episode schizophrenia spectrum disorders at baseline in the Danish OPUS study. Nerv Ment Dis 2007, 195(5):396-405.

73. Weiser M, Reichenberg A, Rabinowitz J, Kaplan Z, Mark M, Nahon D, Davidson M: Gender differences in premorbid cognitive performance in a national cohort of schizophrenic patients. Schizophr Res 2000, 45(3):185-190.

74. Green RE, Melo B, Christensen B, Ngo LA, Monette G, Bradbury C: Measuring premorbid IQ in traumatic brain injury: an examination of the validity of the Wechsler Test of Adult Reading (WTAR). J Clin Exp Neuropsychol 2008, 30(2):163-172.

75. Aylward E, Walker $E$, Bettes $B$ : Intelligence in schizophrenia: meta-analysis of the research. Schizophr Bull 1984, 10(3):430-459.

76. Kern RS, Nuechterlein KH, Green MF, Baade LE, Fenton WS, Gold JM, Keefe RS, Mesholam-Gately R, Mintz J, Seidman LJ, Stover E, Marder SR: The MATRICS Consensus Cognitive Battery, part 2: co-norming and standardization. Am J Psychiatry 2008, 165(2):214-220.

77. Hoff AL, Wieneke M, Faustman WO, Horon R, Sakuma M, Blankfeld H, Espinoza S, DeLisi LE: Sex differences in neurops ychological functioning of first-episode and chronically ill schizophrenic patients. Am J Psychiatry 1998, 155(10):1437-1439.

78. Seeman MV: Gender differences in the prescribing of antipsychotic drugs. Am J Psychiatry 2004, 161(8):1324-1333.

79. Seeman MV: Secondary effects of antipsychotics: women at greater risk than men. Schizophr Bull 2009, 35(5):937-948.

80. Dominguez Mde G, Viechtbauer W, Simons CJ, van Os J, Krabbendam L: Are psychotic psychopathology and neurocognition orthogonal? A systematic review of their associations. Psychol Bull 2009, 135(1):157-171.

81. Puig O, Penades R, Gasto C, Catalan R, Torres A, Salamero M: Verbal memory, negative symptomatology and prediction psychosocial functioning in schizophrenia. Psychiatry Res 2008, 158(1):11-17.

82. Schennach-Wolff $R$, Jager M, Seemuller F, Obermeier M, Messer $T$, Laux G, Pfeiffer H, Naber D, Schmidt LG, Gaebel W, Huff W, Heuser I, Maier W, Lemke MR, Ruther E, Buchkremer G, Gastpar M, Moller HJ, Riedel M: Defining and predicting functional outcome in schizophrenia and schizophrenia spectrum disorders. Schizophr Res 2009, 113(2-3):210-217.
83. Gaite L, Vazquez-Barquero JL, Herran A, Thornicroft G, Becker T, SierraBiddle D, Ruggeri M, Schene A, Knapp M, Vazquez-Bourgon J: Main determinants of Global Assessment of Functioning score in schizophrenia: a European multicenter study. Compr Psychiatry 2005, 46(6):440-446.

84. Ehrenreich $\mathrm{H}$, Siren AL: Neuroprotection-what does it mean?-What means do w e have? Eur Arch Psychiatry Clin Neurosci 2001, 251(4):149-151.

\section{Pre-publication history}

The pre-publication history for this paper can be accessed here: http://www.biomedcentral.com/1471-244X/10/91/prepub

doi:10.1186/1471-244X-10-91

Cite this article as: Ribbe et al:: The cross-sectional GRAS sample: $A$ comprehensive phenotypical data collection of schizophrenic patients. BMC Psychiatry 2010 10:91.

\section{Submit your next manuscript to BioMed Central and take full advantage of:}

- Convenient online submission

- Thorough peer review

- No space constraints or color figure charges

- Immediate publication on acceptance

- Inclusion in PubMed, CAS, Scopus and Google Scholar

- Research which is freely available for redistribution 
Sergi Papiol, ${ }^{1,2,3}$ Martin Begemann, ${ }^{1}$ Albert Rosenberger, ${ }^{4}$ Heidi Friedrichs, ${ }^{1}$ Katja Ribbe,${ }^{1}$ Sabrina Grube, ${ }^{1,3}$ Markus H. Schwab, ${ }^{2,3}$ Henriette Jahn, ${ }^{5}$ Stefan Gunkel, ${ }^{6}$ Fritz Benseler, Klaus-Armin Nave, ${ }^{2,3 *}$ and Hannelore Ehrenreich ${ }^{1,3 *}$

\author{
${ }^{1}$ Division of Clinical Neuroscience, Max Planck Institute of Experimental Medicine, Göttingen, Germany \\ ${ }^{2}$ Department of Neurogenetics, Max Planck Institute of Experimental Medicine, Göttingen, Germany \\ ${ }^{3}$ DFG Research Center for Molecular Physiology of the Brain (CMPB), Göttingen, Germany \\ ${ }^{4}$ Department of Genetic Epidemiology, Georg-August University, Medical School, Göttingen, Germany \\ ${ }^{5}$ Department of Psychiatry II, Ulm University, District Hospital Günzburg, Günzburg, Germany \\ ${ }^{6}$ Hospital of Psychiatry \& Psychotherapy, Langenhagen, Germany \\ 'Department of Molecular Neurobiology, Max Planck Institute of Experimental Medicine, Göttingen, Germany
}

Received 8 October 2010; Accepted ? December 2010

By pure endpoint diagnosis of the disease, the risk of developing schizophrenia has been repeatedly associated with specific variants of the neuregulin1 (NRG1) gene. However, the role of NRG1 in the etiology of schizophrenia has remained unclear. Since Nrg1 serves vital functions in early brain development of mice, we hypothesized that human NRG1 alleles codetermine developmentally influenced readouts of the disease: age of onset and positive symptom severity. We analyzed 1,071 comprehensively phenotyped schizophrenic/schizoaffective patients, diagnosed according to DSM-IV-TR, from the GRAS (Göttingen Research Association for Schizophrenia) Data Collection for genetic variability in the Icelandic region of risk in the NRG1 gene. For the case-control analysis part of the study, we included 1,056 healthy individuals with comparable ethnicity. The phenotype-based genetic association study (PGAS) was performed on the GRAS sample. Instead of a risk constellation, we detected that several haplotypic variants of NRG1 were, unexpectedly, less frequent in the schizophrenic than in the control sample (mean $O R=0.78$, range between 0.68 and 0.85 ). In the PGAS we found that these "protective" NRG1 variants are specifically underrepresented in subgroups of schizophrenic subjects with early age of onset and high positive symptom load. The GRAS Data Collection as a prerequisite for PGAS has enabled us to associate protective NRG1 genotypes with later onset and milder course of schizophrenia.

๑) 2011 Wiley-Liss, Inc.

Key words: NRG1; PGAS; GWAS; protective genotypes; PANSS; data base
How to Cite this Article:

Papiol S, Begemann M, Rosenberger A, Friedrichs H, Ribbe K, Grube S, Schwab MH, Jahn H, Gunkel S, Benseler F, Nave K-A, Ehrenreich H. 2011. A Phenotype-Based Genetic Association Study Reveals the Contribution of Neuregulin1 Gene Variants to Age of Onset and Positive Symptom Severity in Schizophrenia.

Am J Med Genet Part B 9999:1-6.

This Article was published online on 13 Jan 2011. An error was subsequently identified with the Figure Legend text of Figure 1. This notice is included in the online version to indicate that that it has been corrected 27 Jan 2011. The publisher regrets the error.

Additional Supporting Information may be found in the online version of this article.

${ }^{\star}$ Correspondence to:

Klaus-Armin Nave or Hannelore Ehrenreich, Max Planck Institute of Experimental Medicine, Hermann-Rein-Str.3, 37075 Göttingen, Germany. E-mail: nave@em.mpg.de or ehrenreich@em.mpg.de Published online in Wiley Online Library

(wileyonlinelibrary.com)

DOI 10.1002/ajmg.b.31168 


\section{INTRODUCTION}

Schizophrenia is the collective term for a heterogeneous group of severely disabling, chronic mental disorders. They affect approximately $1 \%$ of the population and are characterized by hallucinations, delusions, disordered thought, cognitive impairment, blunted emotions, and subtle motor abnormalities.

The etiology of schizophrenia has remained elusive, even though twin studies have reported heritability estimates between $64 \%$ and $88 \%$, clearly underlining its genetic roots [Cardno and Gottesman, 2000; Lichtenstein et al., 2009]. Following the landmark study of Stefansson et al. [2002], the neuregulin1 gene (NRG1) on chromosome $8 \mathrm{p} 12$ has been consistently associated with the disease in several human populations [Harrison and Law, 2006; Li et al., 2006]. With odds ratios amounting up to 2.2 [Stefansson et al., 2002], NRG1 is among the most prominent risk genes detected so far [Harrison and Law, 2006; Li et al., 2006], and has also been associated in healthy individuals or in relatively small groups of schizophrenic patients with some specific features of the disease, such as white matter anomalies [McIntosh et al., 2008; Sprooten et al., 2009], reduced sensorimotor gating as measured by prepulse inhibition [Hong et al., 2008] or abnormal cortical activation in fronto-temporal areas [Hall et al., 2006].

The NRG1 gene is unusually large $(1.4 \mathrm{Mb})$, comprising $\sim 30$ exons and a complex pattern of differential promoter usage and alternative RNA splicing [Falls, 2003; Steinthorsdottir et al., 2004]. Multiple transcripts encode at least 16 different NRG1 isoforms, including membrane-bound and soluble neuronal growth factors that share the EGF-like signaling domain. Six major NRG1 subtypes can be defined by the presence or absence of transmembrane domains and extracellular matrix attachment sites [Esper et al., 2006]. All NRG1 isoforms signal to ErbB receptor tyrosine kinases expressed by target cells.

In the developing nervous system, NRG1/ErbB4 signaling displays numerous critical functions, including neurogenesis [Ghashghaei et al., 2006], tangential migration of interneurons [Flames et al., 2004; Wen et al., 2010], axon guidance [LopezBendito et al., 2006], synapse formation [Bjarnadottir et al., 2004] and myelination [Brinkmann et al., 2008; Mei and Xiong, 2008]. All of these developmental processes, when quantitatively impaired, have the potential to impact on higher cortical function and therefore make NRG1 a biologically plausible schizophrenia susceptibility gene [Corfas et al., 2004; Mei and Xiong, 2008].

According to previous association studies, the core region conferring the risk to schizophrenia spans about $200 \mathrm{~kb}$ and includes several single nucleotide polymorphisms (SNPs) and microsatellites. Contained in this area is the 5 ' regulatory region upstream of exon 1 and exon 2, which define NRG1 isoforms IV and II, respectively [Harrison and Law, 2006; Munafo et al., 2006].

We have hypothesized that the early developmental functions of $N R G 1$ may explain the specific association of polymorphic markers of NRG1 with developmental features of the disease. To this end, a "phenotype-based genetic association study" (PGAS) was carried out employing the Göttingen Research Association for Schizophrenia (GRAS) Data Collection [Begemann et al., 2010; Ribbe et al., 2010]. This database provides a comprehensive and standardized phenotype characterization of as yet unprecedented detail of
$>1,000$ schizophrenic patients. For exploring the phenotypical consequences of NRG1 genotypes, age of onset of the disease was selected as primary indicator of the degree of developmental disturbance. Further rationales were that an earlier age of onset has been associated (a) with a higher familial/genetic load, independent of any known genetic variants [Kumra and Charles Schulz, 2008], and (b) with greater clinical and psychopathological severity [Rutter et al., 2006], particularly positive symptoms in some studies [Luoma et al., 2008].

Based on the analysis of four single nucleotide polymorphisms (SNPs) and four microsatellites, covering the NRG1 core region of risk, we first tested whether NRG1 is a risk gene in the German GRAS population, when compared to healthy controls. Instead of the expected risk constellation, we detected "protective" NRG1 genotypes that we found underrepresented in patients with early age of onset and high positive symptom severity. These results should be considered in the context of neurodevelopmental functions of this gene.

\section{MATERIALS AND METHODS}

For a more comprehensive version of methods see Supplementary Material. The Göttingen Research Association for Schizophrenia (GRAS) study has been approved by the Ethics Committee of the Georg-August-University of Göttingen, Germany (master committee), and the local internal review boards of all participating centers, and complies with the Helsinki declaration. The GRAS Data Collection, set up as prerequisite for phenotype-based genetic association studies (PGAS), was accumulated between 2005 and 2008 by an invariable team of traveling investigators, and contains presently $>3,000$ data points per subject, including biographic and family information, disease history, environmental risk factors, comorbidities, and treatments. Moreover, it includes the results of cross-sectional psychopathological, neuropsychological, and neurological examinations [Begemann et al., 2010; Ribbe et al., 2010].

\section{Subjects}

Patients. The GRAS project comprises at present 1,071 patients of 23 German psychiatric hospitals (Supplementary Table S1), diagnosed according to DSM-IV-TR [Wittchen et al., 1997; Association, 2000] with schizophrenia (78.0\%; $\mathrm{N}=835)$, schizoaffective disorder $(17.1 \% ; \mathrm{N}=183)$ and other psychotic disorders/yet to be confirmed $(4.9 \% ; \mathrm{N}=53)$. Patients were included regardless of the stage of the disease (acute, chronic, residual, or remitted). Subjects had to be at least 18 years old; there was no upper age limit. All study participants and, if applicable, their legal representatives gave written informed consent [for more detailed information on the GRAS sample see Begemann et al., 2010; Ribbe et al., 2010]. Almost all of them were of European Caucasian descent (Caucasian 95.3\%; other ethnicities 1.6\%; unknown $3.1 \%$ ). The average age was $39.62 \pm 12.76$ years, with a range from 18 to 83 years. Men $(n=714 ; 66.7 \%)$ were $37.57 \pm 12.00$, women $(\mathrm{n}=357 ; 33.3 \%) 43.74 \pm 13.26$ years old.

Control subjects $(\mathrm{N}=1,056)$ were voluntary blood donors, recruited by the Department of Transfusion Medicine at the Georg-August-University of Göttingen, according to national guidelines for blood donation. As such, they widely fulfill health criteria, ensured by a broad pre-donation screening process con- 
taining standardized questionnaires, interviews, hemoglobin and other blood parameters, blood pressure, pulse, and body temperature determinations [Begemann et al., 2010]. Of the 1,056 subjects, $58.4 \%$ were male $(\mathrm{N}=617)$ and $41.6 \%$ female $(\mathrm{N}=439)$. The average age was $34.72 \pm 12.33$ years, with a range from 18 to 69 years. Comparable to the patient population, almost all control subjects were of European Caucasian descent (Caucasian 97.8\%; other ethnicities $2 \%$; unknown $0.2 \%$ ). All donors gave written informed consent.

\section{Selected Phenotypes}

Of all the variables available for each subject, age of onset and psychopathology were exclusively selected as the phenotypes of interest. Age of onset was defined as the age when the first psychotic episode took place. Severity of psychopathology was ascertained by means of Positive and Negative Syndrome Scale (PANSS) [Kay et al., 1987]. To further validate the results of this hypothesis-driven study, 2 control variables were introduced. As disease-related and disease-unrelated control variables, basic cognition/fine motor function (MacQuarrie Tapping [Chapman, 1948]) and body length were used, respectively.

\section{Genetic Analyses}

To cover the previously described Icelandic haplotype of risk locus [Stefansson et al., 2002] in the neuregulin1 gene (NRG1; Unigene Hs.453951), four SNPs (SNP8NRG221132, SNP8NRG221533, SNP8NRG241930, and SNP8NRG243177) and four microsatellites (MS487-2, MS478B14-848, MS420M9-1395, and D8S1810), spanning a region of $\sim 220 \mathrm{~kb}$ were selected for the analysis. SNP genotyping was performed with either HybProbes or SimpleProbes (TIB Molbiol, Berlin, Germany) on the Light Cycler480 (Roche, Mannheim, Germany). Multiplex microsatellite genotyping was carried out on a 3730XL DNA Analyzer (Applied Biosystems, Foster City, CA).

\section{Statistical Analyses}

Statistical analyses of the effect of genetic variability on selected readouts were performed using SPSS for Windows version 17.0 (https://www.spss.com/de) and SAS v9.2 (http://www.sas.com/offices/europe/germany/index.html). Age of onset underwent a logarithmic transformation to achieve approximate normally distributed values. Haplotype association analyses of binary categorical variables and estimation of linkage disequilibrium $D^{\prime}$ were performed with UNPHASED (v3.0.13). UNPHASED is an application for performing genetic association analysis in nuclear families and unrelated subjects. It implements maximum-likelihood inference on haplotype and genotype effects while allowing for uncertain phase and missing genotypes [Dudbridge, 2008]. For consistency/simplification, the four microsatellites were transformed into "pseudo-SNPs" by classifying each allele in short or long (length shorter or longer than the mean repeat length; see Supplementary Table S2) for some of the analyses. Genotypic association of SNPs and "pseudo-SNPs" in the case-control study was assessed by two-sided Cochran-Armitage trend tests, while the full range of genotypes observed in microsatellites was analyzed by two-sided nonparametric two-sample Kolmogórov-Smirnov tests. The association to ordinal scaled disease phenotypes (age of onset and PANSS positive score) was assessed by one-sided JonckheereTerpstra trend test and Wilcoxon rank sum test. The same procedures were employed to further check the specificity of the statistical results by evaluating disease-related (basic cognition/fine motor function) and disease-unrelated (body length) control variables. These control variables were grouped into quartiles for analysis.

\section{RESULTS}

All single markers fulfilled the Hardy-Weinberg equilibrium criteria using a $P<0.01$ threshold (Supplementary Table S3). The markers showed a strong to moderate degree of linkage disequilibrium between them (Supplementary Table S4). The single-marker case-control study did not yield any association with the disease, nor did it reveal gender differences (Supplementary Table S5). Likewise, haplotypic analysis failed to replicate in the GRAS patients the NRG1 at-risk haplotype finding previously described in the Icelandic population.

However, the same analysis revealed a "protective" constellation of NRG1 alleles cutting across several different haplotypic combinations (mean $\mathrm{OR}=0.78$, range between 0.68 and 0.85) (Fig. 1 and Supplementary Fig. S1), and partly opposite to the allelic risk variants (in three of the four analyzed SNPs) described by Stefansson et al. [2002]. For the analyzed microsatellites, the protective variants were essentially also opposite to those contained in the Icelandic risk haplotype.

Following our hypothesis that genetic variants of NRG1 are associated with the developmental profile of schizophrenia, we analyzed the frequency distribution of these markers in subsets of patients, defined by age of onset. This PGAS approach revealed that several protective genetic variants in homozygosity were underrepresented in the group of patients showing early age of onset ( $\leq 20$ years) and also - to a lesser degree - in the group of schizophrenic individuals with most severe positive symptomatology (PANSS positive score higher than 18) (Fig. 1 and Supplementary Tables S6, S7a,b). The control variables basic cognition/fine motor function and body length did not show any association with NRG1 genotypes (all $P$ values $>0.05$, Supplementary Table S8).

\section{DISCUSSION}

The present study has identified a "protective" role of NRG1 gene variants specifically influencing age of onset and severity of positive symptoms in the GRAS sample of schizophrenic patients. Even though previous work also described a protective constellation in Swedish and Korean populations based on case-control comparison [Kim et al., 2006; Alaerts et al., 2009], the protective effect itself had not been associated with any phenotypical consequences.

By meta-analyses, NRG1 has been confirmed as a susceptibility gene for schizophrenia in some but not all populations [ $\mathrm{Li}$ et al., 2006; Munafo et al., 2006]. The negative finding with respect to the risk role of NRG1 presented here using the GRAS cohort may be explained by population differences, by sample size, or both. In contrast, PGAS has been successfully used in our cohort of $>1,000$ 


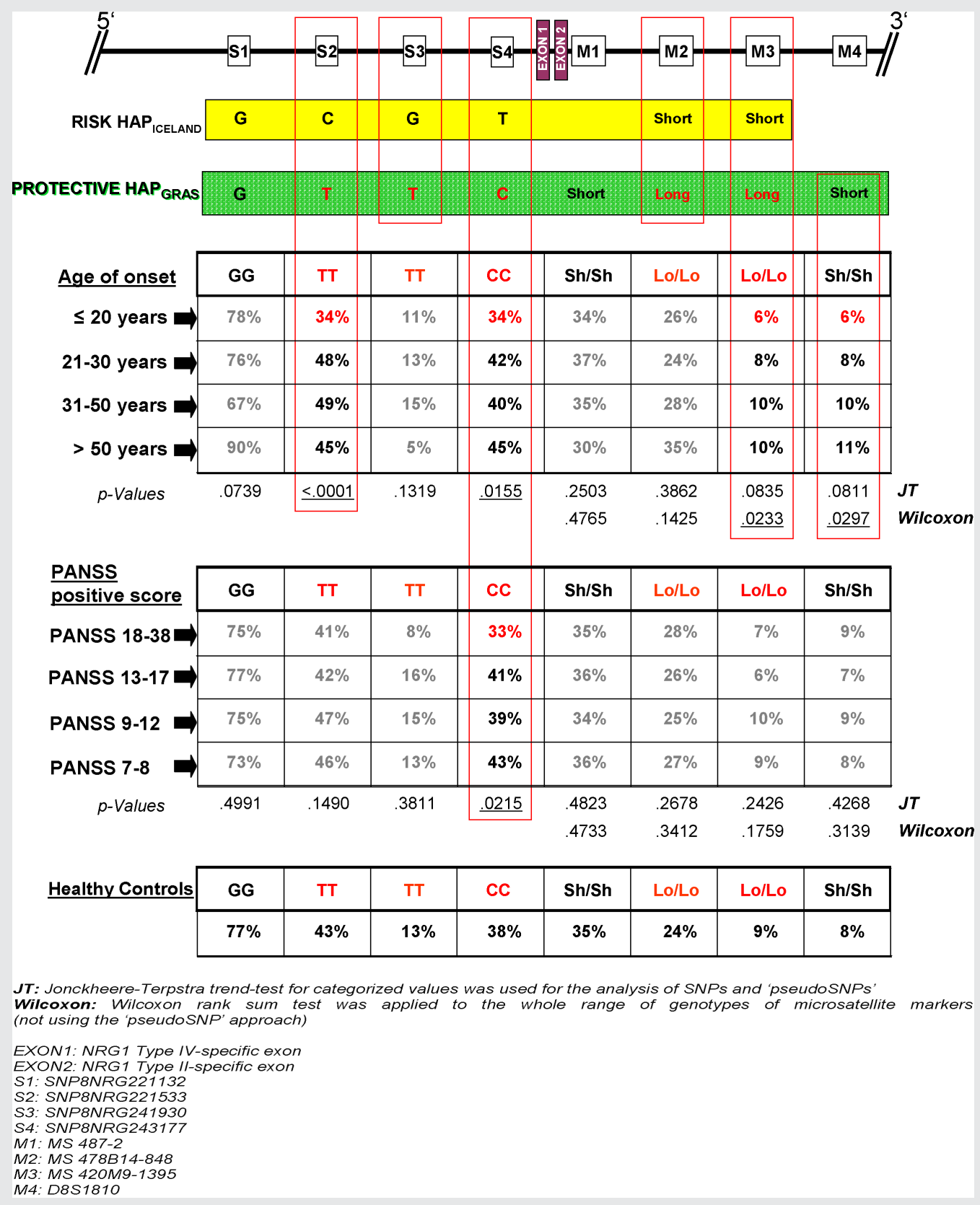

FIG. 1. Schematic view of the $220 \mathrm{~kb}$ region of interest at the $5^{\prime}$ region of the NRG1 gene. The Icelandic risk haplotype and its associated risk alleles are given. Underneath, the German [GRAS] protective haplotype is presented. Several haplotypic combinations which contain the opposite alleles of the risk constellation (see vertical squares] in markers S2, S3, S4, M2, and M3 are more frequent in controls than in the GRAS patients [see Supplementary Fig. S1). At least for some markers, the protective genotypes are underrepresented in early onset cases and in patients with most severe positive symptomatology. $P$-values refer to comparisons of patients with an early onset of the disease $[\leq \mathbf{2 0}$ years] to the remaining patients. Likewise, patients with a severe positive psychopathology (score $\geq 18$ ) are compared with the other patients. Frequency of protective genotypes in the total healthy German [GRAS) control population is shown at the bottom table.

patients to reveal that some NRG1 gene variants have a "protective" feature, as suggested by homozygous carriers being underrepresented among patients with early onset and severe positive symptoms. Interestingly, these markers are opposed to or overlap, but are not identical with those that provide a higher risk for schizophrenia by simple endpoint diagnosis in other studies. We note that the Icelandic haplotype could not be associated with the age of onset in a much smaller sample of patients [Voineskos et al., 2009].

Interestingly, marker S4 (SNP8NRG243177), influencing both age of onset and severity of positive symptoms in our cohort, regulates the expression of the brain-specific NRG1 isoform type IV, as shown by genotype-dependent mRNA expression in transfected cells (luciferase assays) [Tan et al., 2007] as well as in human 
post mortem samples [Law et al., 2006]. This would suggest that altered (decreased) NRG1 expression might be protective in the here reported context. However, RNA steady state levels in autopsy material obtained from adult patients [Hahn et al., 2006; Law et al., 2006] cannot predict the impact of NRG1 gene expression levels in all neurons or at all stages of brain development.

We have proposed that unfavorable fine-tuning of NRG1 expression and/or function during development due to genetic variability is specifically connected with earlier onset of schizophrenia and severity of psychopathology, which has in principle been confirmed by our findings. At first glance, the model of a "protective" NRG1 allele, that is, involving a gene that is considered as a "risk factor," may appear paradoxical. However, as mentioned above, two other studies, comparing cases and controls, have also reported "protective" haplotypes in NRG1 [Kim et al., 2006; Alaerts et al., 2009].

We suggest that the PGAS approach, in combination with preclinical data on Nrgl function in mouse models, can shed light onto this puzzle, and may also help explaining the difficulties of confirming the original association. NRG1/ErbB signaling has distinct roles, both in brain development and in adult cognitive functions, all of which are relevant to schizophrenia, but differently perturbed by loss- and gain-of-function effects. For example, NRG1/ErbB4 signaling has been directly implicated in the intracortical migration of GABAergic interneurons and the refinement of inhibitory synapses [Flames et al., 2004; Fazzari et al., 2010]. Altered expression of NRG1 isoforms, the proposed consequence of the NRG1 "at risk" or "protective" alleles, might therefore affect the number and function of inhibitory cortical interneurons. This could plausibly accelerate/delay the onset of a disease, in which cortical inhibition fails [Lewis et al., 2005]. On the other hand, NRG1/ErbB4 signaling attenuates glutamatergic neurotransmission in the mature cortex [Mei and Xiong, 2008; Buonanno, 2010], and it is equally plausible that quantitatively changed NRG1 ultimately enhances cognitive dysfunction and other symptoms that collectively result in the diagnosis of schizophrenia.

To conclude, our work associates for the first time in a large population of $>1,000$ schizophrenic patients clinically relevant phenotypes with NRG1 gene variants. In fact, distinct NRG1 variants are underrepresented in early onset and severe cases of schizophrenia, delivering a phenotypical readout for the protective constellation as derived from our case-control analysis. Replication of these results in independent samples would be desirable as well as analysis of genetic/haplotypic variants in regions of the gene that have not been considered in the present study. Moreover, further studies-also involving more mechanism-targeted animal models-are needed in order to understand the molecular basis of the dual risk/protective role of NRG1.

\section{ACKNOWLEDGMENTS}

None of the authors has a direct or indirect conflict of interest to declare. This study has been supported by the Max Planck Society and the DFG Research Center for Molecular Physiology of the Brain (CMPB). Sergi Papiol was supported by a short-term fellowship from the Alicia Koplowitz Foundation and a postdoctoral fellowship of the Spanish MEC (2007-0280). The authors thank all patients for their participation in this study, and all collaborating centers for their support. Dr. Andrés Ingason is acknowledged for providing information on markers 478B14-848 and 420M9-1395 in an Icelandic population.

\section{REFERENCES}

Alaerts M, Ceulemans S, Forero D, Moens LN, De Zutter S, Heyrman L, Lenaerts AS, Norrback KF, De Rijk P, Nilsson LG, et al. 2009. Support for NRG1 as a susceptibility factor for schizophrenia in a northern Swedish isolated population. Arch Gen Psychiatry 66(8):828-837.

Association AP. 2000. Diagnostic and statistical manual of mental disorders: DSM-IV-TR. Washington: American Psychiatric Association.

Begemann M, Grube S, Papiol S, Malzahn D, Krampe H, Ribbe K, Friedrichs H, Radyushkin KA, El-Kordi A, Benseler F, et al. 2010. Modification of cognitive performance in schizophrenia by complexin 2 gene polymorphisms. Arch Gen Psychiatry 67(9):879-888.

Bjarnadottir TK, Fredriksson R, Hoglund PJ, Gloriam DE, Lagerstrom MC, Schioth HB. 2004. The human and mouse repertoire of the adhesion family of G-protein-coupled receptors. Genomics 84(1):23-33.

Brinkmann BG, Agarwal A, Sereda MW, Garratt AN, Muller T, Wende H, Stassart RM, Nawaz S, Humml C, Velanac V, et al. 2008. Neuregulin-1/ ErbB signaling serves distinct functions in myelination of the peripheral and central nervous system. Neuron 59(4):581-595.

Buonanno A. 2010. The neuregulin signaling pathway and schizophrenia: From genes to synapses and neural circuits. Brain Res Bull 83(3-4): $122-131$.

Cardno AG, Gottesman II. 2000. Twin studies of schizophrenia: From bowand-arrow concordances to star wars $\mathrm{Mx}$ and functional genomics. Am J Med Genet 97(1):12-17.

Chapman RL. 1948. The MacQuarrie test for mechanical ability. Psychometrika 13(3):175-179.

Corfas G, Roy K, Buxbaum JD. 2004. Neuregulin 1-erbB signaling and the molecular/cellular basis of schizophrenia. Nat Neurosci 7(6): 575-580.

Dudbridge F. 2008. Likelihood-based association analysis for nuclear families and unrelated subjects with missing genotype data. Hum Hered 66(2):87-98.

Esper RM, Pankonin MS, Loeb JA. 2006. Neuregulins: Versatile growth and differentiation factors in nervous system development and human disease. Brain Res Rev 51(2):161-175.

Falls DL. 2003. Neuregulins: Functions, forms, and signaling strategies. Exp Cell Res 284(1):14-30.

Fazzari P, Paternain AV, Valiente M, Pla R, Lujan R, Lloyd K, Lerma J, Marin O, Rico B. 2010. Control of cortical GABA circuitry development by Nrg1 and ErbB4 signalling. Nature 464(7293):1376-1380.

Flames N, Long JE, Garratt AN, Fischer TM, Gassmann M, Birchmeier C, Lai C, Rubenstein JL, Marin O. 2004. Short- and long-range attraction of cortical GABAergic interneurons by neuregulin-1. Neuron 44(2): 251-261.

Ghashghaei HT, Weber J, Pevny L, Schmid R, Schwab MH, Lloyd KC, Eisenstat DD, Lai C, Anton ES. 2006. The role of neuregulin-ErbB4 interactions on the proliferation and organization of cells in the subventricular zone. Proc Natl Acad Sci USA 103(6):1930-1935.

Hahn CG, Wang HY, Cho DS, Talbot K, Gur RE, Berrettini WH, Bakshi K, Kamins J, Borgmann-Winter KE, Siegel SJ, et al. 2006. Altered neuregulin 1-erbB4 signaling contributes to NMDA receptor hypofunction in schizophrenia. Nat Med 12(7):824-828. 
Hall J, Whalley HC, Job DE, Baig BJ, McIntosh AM, Evans KL, Thomson PA, Porteous DJ, Cunningham-Owens DG, Johnstone EC, et al. 2006. A neuregulin 1 variant associated with abnormal cortical function and psychotic symptoms. Nat Neurosci 9(12):1477-1478.

Harrison PJ, Law AJ. 2006. Neuregulin 1 and schizophrenia: Genetics, gene expression, and neurobiology. Biol Psychiatry 60(2):132-140.

Hong LE, Wonodi I, Stine OC, Mitchell BD, Thaker GK. 2008. Evidence of missense mutations on the neuregulin 1 gene affecting function of prepulse inhibition. Biol Psychiatry 63(1):17-23.

Kay SR, Fiszbein A, Opler LA. 1987. The positive and negative syndrome scale (PANSS) for schizophrenia. Schizophr Bull 13(2):261-276.

Kim JW, Lee YS, Cho EY, Jang YL, Park DY, Choi KS, Jeun HO, Cho SH, Jang SY, Hong KS. 2006. Linkage and association of schizophrenia with genetic variations in the locus of neuregulin 1 in Korean population. Am J Med Genet Part B 141B(3):281-286.

Kumra S, Charles Schulz S. 2008. Editorial: Research progress in early-onset schizophrenia. Schizophr Bull 34(1):15-17.

Law AJ, Lipska BK, Weickert CS, Hyde TM, Straub RE, Hashimoto R, Harrison PJ, Kleinman JE, Weinberger DR. 2006. Neuregulin 1 transcripts are differentially expressed in schizophrenia and regulated by $5^{\prime}$ SNPs associated with the disease. Proc Natl Acad Sci USA 103(17): 6747-6752.

Lewis DA, Hashimoto T, Volk DW. 2005. Cortical inhibitory neurons and schizophrenia. Nat Rev Neurosci 6(4):312-324.

Li D, Collier DA, He L. 2006. Meta-analysis shows strong positive association of the neuregulin 1 (NRG1) gene with schizophrenia. Hum Mol Genet 15(12):1995-2002.

Lichtenstein P, Yip BH, Bjork C, Pawitan Y, Cannon TD, Sullivan PF, Hultman CM. 2009. Common genetic determinants of schizophrenia and bipolar disorder in Swedish families: A population-based study. Lancet 373(9659):234-239.

Lopez-Bendito G, Cautinat A, Sanchez JA, Bielle F, Flames N, Garratt AN, Talmage DA, Role LW, Charnay P, Marin O, et al. 2006. Tangential neuronal migration controls axon guidance: A role for neuregulin-1 in thalamocortical axon navigation. Cell 125(1):127-142.

Luoma S, Hakko H, Ollinen T, Jarvelin MR, Lindeman S. 2008. Association between age at onset and clinical features of schizophrenia: The Northern Finland 1966 birth cohort study. Eur Psychiatry 23(5): 331-335.

McIntosh AM, Moorhead TW, Job D, Lymer GK, Munoz Maniega S, McKirdy J, Sussmann JE, Baig BJ, Bastin ME, Porteous D, et al. 2008. The effects of a neuregulin 1 variant on white matter density and integrity. Mol Psychiatry 13(11):1054-1059.

Mei L, Xiong WC. 2008. Neuregulin 1 in neural development, synaptic plasticity and schizophrenia. Nat Rev Neurosci 9(6):437-452.

Munafo MR, Thiselton DL, Clark TG, Flint J. 2006. Association of the NRG1 gene and schizophrenia: A meta-analysis. Mol Psychiatry 11(6):539-546.

Ribbe K, Friedrichs H, Begemann M, Grube S, Papiol S, Kastner A, Gerchen MF, Ackermann V, Tarami A, Treitz A, et al. 2010. The cross-sectional GRAS sample: A comprehensive phenotypical data collection of schizophrenic patients. BMC Psychiatry 10(1):91.

Rutter M, Kim-Cohen J, Maughan B. 2006. Continuities and discontinuities in psychopathology between childhood and adult life. J Child Psychol Psychiatry 47(3-4):276-295.

Sprooten E, Lymer GK, Munoz Maniega S, McKirdy J, Clayden JD, Bastin ME, Porteous D, Johnstone EC, Lawrie SM, Hall J, et al. 2009. The relationship of anterior thalamic radiation integrity to psychosis risk associated neuregulin-1 variants. Mol Psychiatry 14(3):237-238, 233.

Stefansson H, Sigurdsson E, Steinthorsdottir V, Bjornsdottir S, Sigmundsson T, Ghosh S, Brynjolfsson J, Gunnarsdottir S, Ivarsson O, Chou TT, et al. 2002. Neuregulin 1 and susceptibility to schizophrenia. Am J Hum Genet 71(4):877-892.

Steinthorsdottir V, Stefansson H, Ghosh S, Birgisdottir B, Bjornsdottir S, Fasquel AC, Olafsson O, Stefansson K, Gulcher JR. 2004. Multiple novel transcription initiation sites for NRG1. Gene 342(1):97-105.

Tan W, Wang Y, Gold B, Chen J, Dean M, Harrison PJ, Weinberger DR, Law AJ. 2007. Molecular cloning of a brain-specific, developmentally regulated neuregulin 1 (NRG1) isoform and identification of a functional promoter variant associated with schizophrenia. J Biol Chem 282(33): 2434324351.

Voineskos D, De Luca V, Macgregor S, Likhodi O, Miller L, Voineskos AN, Kennedy JL. 2009. Neuregulin 1 and age of onset in the major psychoses. J Neural Transm 116(4):479-486.

Wen L, Lu YS, Zhu XH, Li XM, Woo RS, Chen YJ, Yin DM, Lai C, Terry AV, Jr., Vazdarjanova A, et al. 2010. Neuregulin 1 regulates pyramidal neuron activity via ErbB4 in parvalbumin-positive interneurons. Proc Natl Acad Sci USA 107(3):1211-1216.

Wittchen H-U, Zaudig M, Fydrich T. 1997. SKID-I (Strukturiertes Klinisches Interview für DSM-IV; Achse I: Psychische Störungen). Göttingen: Hogrefe. 


\title{
Supplementary Material
}

\begin{abstract}
A phenotype-based genetic association study reveals the contribution of neuregulin1 gene variants to age of onset and positive symptom severity in schizophrenia
\end{abstract}

Sergi Papiol PhD ${ }^{1,2,7}$; Martin Begemann MD¹; Albert Rosenberger MA ${ }^{3}$;

Heidi Friedrichs $\mathrm{MSc}^{1}$; Katja Ribbe $\mathrm{MSc}^{1}$; Sabrina Grube $\mathrm{MSc}^{1,7}$; Markus H Schwab $\mathrm{PhD}^{2,7}$; Henriette Jahn $\mathrm{MD}^{4}$; Stefan Gunkel $\mathrm{PhD}^{5}$; Fritz Benseler $\mathrm{BSc}^{6}$; Klaus-Armin Nave $\mathrm{PhD}^{2,7 \S}$ and Hannelore Ehrenreich MD, DVM ${ }^{1,7} \S$

\section{Outline:}

Supplementary Tables

Supplementary Figure

Supplementary Methods

Supplementary Material - References 


\section{Supplementary Tables}

Table S1.

Collaborating Centers

\begin{tabular}{|c|c|c|c|}
\hline & Center & Institution & Head \\
\hline 1 & $\begin{array}{l}\text { Bad Emstal - } \\
\text { Merxhausen }\end{array}$ & $\begin{array}{l}\text { Vitos Hospital of Psychiatry and } \\
\text { Psychotherapy, Bad Emstal-Merxhausen }\end{array}$ & $\begin{array}{l}\text { Heinrich Kunze } \\
\text { Michael Franz }\end{array}$ \\
\hline 2 & $\begin{array}{l}\text { Bad } \\
\text { Zwischenahn }\end{array}$ & $\begin{array}{l}\text { Karl-Jaspers-Hospital, } \\
\text { Psychiatric Federation Oldenburger Land }\end{array}$ & $\begin{array}{l}\text { Marianne Becker-Emner } \\
\text { Dunja Hinze-Selch }\end{array}$ \\
\hline 3 & Bonn & $\begin{array}{l}\text { Department of Psychiatry and Psychotherapy, } \\
\text { University Medical Center of Bonn }\end{array}$ & Wolfgang Maier \\
\hline 4 & Eltville-Eichberg & Vitos Hospital of Forensic Psychiatry Eltville & Roland Freese \\
\hline 5 & Fulda & $\begin{array}{l}\text { Department of Psychiatry and Psychotherapy, } \\
\text { Hospital Fulda }\end{array}$ & $\begin{array}{l}\text { Adelheid Czernik } \\
\text { Georg Wiedemann }\end{array}$ \\
\hline 6 & Giessen & $\begin{array}{l}\text { Vitos Haina Forensic Psychiatry Hospital, } \\
\text { Haina, Giessen }\end{array}$ & $\begin{array}{l}\text { Rüdiger } \\
\text { Müller-Isberner }\end{array}$ \\
\hline $7 a$ & Göttingen & Department of Transfusion Medicine & Joachim Riggert \\
\hline $7 b$ & Göttingen & $\begin{array}{l}\text { Department of Psychiatry and Psychotherapy, } \\
\text { University Medical Center of Göttingen }\end{array}$ & $\begin{array}{l}\text { Peter Falkai } \\
\text { Eckart Rüther }\end{array}$ \\
\hline 8 & Günzburg & $\begin{array}{l}\text { Department of Psychiatry II, Ulm University, } \\
\text { District Hospital Günzburg }\end{array}$ & Thomas Becker \\
\hline 9 & Hofgeismar & $\begin{array}{l}\text { Vitos Hospital of Psychiatry and } \\
\text { Psychotherapy Merxhausen, Hofgeismar }\end{array}$ & Andreas Mielke \\
\hline 10 & Ingolstadt & $\begin{array}{l}\text { Department of Psychiatry and Psychotherapy, } \\
\text { Hospital Ingolstadt }\end{array}$ & Thomas Pollmächer \\
\hline 11 & Kassel & $\begin{array}{l}\text { Vitos Hospital of Psychiatry and } \\
\text { Psychotherapy Merxhausen, Kassel }\end{array}$ & Rolf Günther \\
\hline 12 & Kiel & $\begin{array}{l}\text { Hospital of Psychiatry and Psychotherapy, } \\
\text { Center for Integrative Psychiatry }\end{array}$ & Josef B. Aldenhoff \\
\hline 13 & Langenhagen & $\begin{array}{l}\text { Hospital of Psychiatry and Psychotherapy } \\
\text { Langenhagen, Regional Hospitals Hanover }\end{array}$ & Gunther Kruse \\
\hline 14 & Liebenburg & Dr. K. Fontheim's Hospital for Mental Health & Frank-Gerald Pajonk \\
\hline 15 & Lübbecke & $\begin{array}{l}\text { Department of Psychiatry and Psychotherapy, } \\
\text { Hospital Lübbecke }\end{array}$ & Udo Schneider \\
\hline 16 & Moringen & Hospital of Forensic Psychiatry & $\begin{array}{l}\text { Martin Schott } \\
\text { Dirk Hesse }\end{array}$ \\
\hline 17 & Mühlhausen & $\begin{array}{l}\text { Department of Psychiatry and Psychotherapy, } \\
\text { Ecumenical Hospital Hainich }\end{array}$ & Lothar Adler \\
\hline 18 & Rickling & Hospital of Psychiatry and Psychotherapy & $\begin{array}{l}\text { Hans-J.Schwarz } \\
\text { Wolfram Schreiber }\end{array}$ \\
\hline 19 & Rieden & Addiction Hospital "Am Waldsee" & Frank Löhrer \\
\hline 20 & Rostock & $\begin{array}{l}\text { Department of Psychiatry and Psychotherapy, } \\
\text { University of Rostock }\end{array}$ & Sabine Herpertz \\
\hline 21 & Taufkirchen & $\begin{array}{l}\text { Department of Psychiatry and Psychotherapy, } \\
\text { Isar-Amper-Hospital, Taufkirchen (Vils) }\end{array}$ & Matthias Dose \\
\hline 22 & Wilhelmshaven & $\begin{array}{l}\text { Department of Psychiatry and Psychotherapy, } \\
\text { Reinhard-Nieter Hospital }\end{array}$ & Here Folkerts \\
\hline 23 & Wunstorf & $\begin{array}{l}\text { Department of Psychiatry and Psychotherapy, } \\
\text { Regional Hospitals Hanover }\end{array}$ & $\begin{array}{l}\text { Andreas Spengler } \\
\text { Cornelia Oestereich }\end{array}$ \\
\hline
\end{tabular}




\section{Table S2.}

Mean allelic repeat marker lengths for transforming microsatellites into 'pseudoSNPs' defined by 'Short' or 'Long' alleles

\begin{tabular}{|c|c|c|c|c|}
\hline & \multirow[b]{2}{*}{ Marker } & \multirow{2}{*}{$\begin{array}{l}\text { Mean allelic } \\
\text { repeat marker } \\
\text { length: definition } \\
\text { of Short versus } \\
\text { Long alleles }\end{array}$} & \multicolumn{2}{|c|}{ Range of repeat lengths } \\
\hline & & & Min & Max \\
\hline M1 & MS 487-2 & Short $<9.11<$ Long & 2 & 16 \\
\hline M2 & MS 478B14-848 & Short $<5.78<$ Long & 2 & 16 \\
\hline M3 & MS 420M9-1395 & Short< $8.29<$ Long & 2 & 20 \\
\hline M4 & D8S1810 & Short $<15.16<$ Long & 2 & 30 \\
\hline
\end{tabular}




\section{Table S3.}

Hardy-Weinberg equilibrium (HWE) ascertainment in the 4 SNPs and the 4 microsatellites transformed into 'pseudoSNPs' (Table S2) in the GRAS sample and healthy controls

\begin{tabular}{|c|c|c|c|c|c|c|c|c|}
\hline \multirow{2}{*}{\multicolumn{2}{|c|}{ Marker }} & \multicolumn{2}{|c|}{ Alleles } & \multirow{2}{*}{$\boldsymbol{N}$} & \multicolumn{3}{|c|}{ Genotype frequency } & \multirow{2}{*}{$\begin{array}{c}\text { HWE } \\
p \text {-Value }\end{array}$} \\
\hline & & Min* & $M a j^{*}$ & & $M a j^{*} / M^{*}{ }^{*}$ & Maj*/Min* & $\operatorname{Min} * / \operatorname{Min}^{*}$ & \\
\hline \multicolumn{9}{|c|}{ Healthy controls } \\
\hline s1 & SNP8NRG221132 & A & G & 1050 & $813(77 \%)$ & $221(21 \%)$ & $16(2 \%)$ & .9758 \\
\hline S2 & SNP8NRG221533 & C & $\mathrm{T}$ & 1053 & $455(43 \%)$ & $474(45 \%)$ & $124(12 \%)$ & .9994 \\
\hline S3 & SNP8NRG241930 & $\mathrm{T}$ & G & 1055 & $429(41 \%)$ & $490(46 \%)$ & $136(13 \%)$ & .9776 \\
\hline S4 & SNP8NRG243177 & $\mathrm{T}$ & C & 1054 & $404(38 \%)$ & $496(47 \%)$ & $154(15 \%)$ & .9961 \\
\hline M1 & SNP_MS 487-2 & Long & Short & 1055 & $378(36 \%)$ & $499(47 \%)$ & $178(17 \%)$ & .8304 \\
\hline M2 & SNP_MS 478B14-848 & Short & Long & 1055 & $253(24 \%)$ & $568(54 \%)$ & $234(22 \%)$ & .0433 \\
\hline M3 & SNP_MS 420M9-1395 & Long & Short & 1053 & $525(50 \%)$ & $429(41 \%)$ & $99(9 \%)$ & .7057 \\
\hline M4 & SNP_D8S1810 & Short & Long & 1050 & $572(54 \%)$ & $397(38 \%)$ & $81(8 \%)$ & .5806 \\
\hline \multicolumn{9}{|c|}{ Schizophrenic patients } \\
\hline S1 & SNP8NRG221132 & A & G & 1051 & $790(75 \%)$ & $247(24 \%)$ & $14(1 \%)$ & .5568 \\
\hline S2 & SNP8NRG221533 & C & $\mathrm{T}$ & 1051 & $464(44 \%)$ & $468(45 \%)$ & $119(12 \%)$ & .9981 \\
\hline S3 & SNP8NRG241930 & $\mathrm{T}$ & G & 1053 & $434(41 \%)$ & $486(46 \%)$ & $133(13 \%)$ & .9857 \\
\hline S4 & SNP8NRG243177 & $\mathrm{T}$ & C & 1050 & $408(39 \%)$ & $490(47 \%)$ & $152(14 \%)$ & .9695 \\
\hline M1 & $\mathrm{SNP}_{-} \mathrm{MS} 487-2$ & Long & Short & 1049 & $372(35 \%)$ & $517(49 \%)$ & $160(15 \%)$ & .6691 \\
\hline M2 & $\mathrm{SNP}_{-}$MS 478B14-848 & Long & Short & 1048 & $274(26 \%)$ & $513(49 \%)$ & $261(25 \%)$ & .7964 \\
\hline M3 & SNP_MS 420M9-1395 & Long & Short & 1047 & $535(51 \%)$ & $426(41 \%)$ & $86(8 \%)$ & .9957 \\
\hline M4 & SNP_D8S1810 & Short & Long & 1018 & $567(56 \%)$ & $368(36 \%)$ & $83(8 \%)$ & 1099 \\
\hline
\end{tabular}

Min $^{*}=$ Minor allele; Maj*$^{*}=$ Major allele 


\section{Table S4.}

Linkage disequilibrium measure (D') in the healthy control sample with respect to the genetic markers analyzed in this study

\begin{tabular}{|c|c|c|c|c|c|c|c|c|}
\hline Marker & 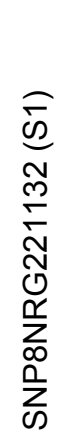 & 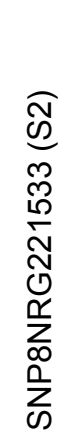 & 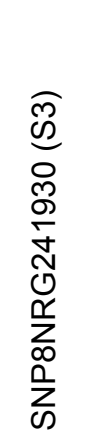 & 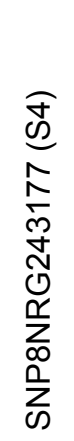 & 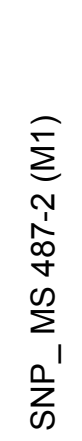 & 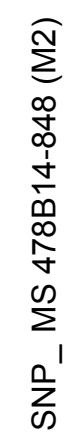 & 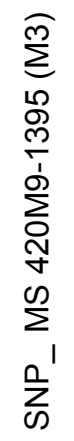 & $\begin{array}{l}\widehat{\Im} \\
\sum \\
0 \\
\infty \\
\infty \\
\infty \\
0 \\
0 \\
\sum_{\infty}^{\prime}\end{array}$ \\
\hline SNP8NRG221132 (S1) & - & 1 & 1 & .94 & .53 & .44 & .47 & .49 \\
\hline SNP8NRG221533 (S2) & & - & .86 & .88 & .37 & .25 & .26 & .26 \\
\hline SNP8NRG241930 (S3) & & & - & .98 & .34 & .40 & .27 & .27 \\
\hline SNP8NRG243177 (S4) & & & & - & .31 & .25 & .25 & .24 \\
\hline SNP_ MS 487-2 (M1) & & & & & - & .67 & .82 & .64 \\
\hline SNP_MS 478B14-848 (M2) & & & & & & - & .76 & .69 \\
\hline SNP_MS 420M9-1395 (M3) & & & & & & & - & .70 \\
\hline SNP_D8S1810 (M4) & & & & & & & & - \\
\hline
\end{tabular}




\section{Table S5.}

Single-marker case-control genotypic association study and sex differences study

\begin{tabular}{|c|c|c|c|}
\hline & \multirow[b]{2}{*}{ Marker } & \multicolumn{2}{|c|}{$p$-Value } \\
\hline & & C-A Trend & K-S \\
\hline \multicolumn{4}{|c|}{ Case-control study } \\
\hline S1 & SNP8NRG221132 & .3625 & \\
\hline S2 & SNP8NRG221533 & .7289 & \\
\hline S3 & SNP8NRG241930 & .9783 & \\
\hline S4 & SNP8NRG243177 & .9597 & \\
\hline M1 & $\mathrm{SNP}_{-} \mathrm{MS} 487-2$ & .7323 & 1.0000 \\
\hline M2 & SNP_ MS 478B14-848 & .3414 & .9594 \\
\hline M3 & $\mathrm{SNP}_{-}$MS 420M9-1395 & .3977 & .9866 \\
\hline M4 & SNP_D8S1810 & .9897 & .6688 \\
\hline \multicolumn{4}{|c|}{ Sex differences study } \\
\hline S1 & SNP8NRG221132 & .5819 & \\
\hline S2 & SNP8NRG221533 & .9714 & \\
\hline S3 & SNP8NRG241930 & .3054 & \\
\hline S4 & SNP8NRG243177 & .9703 & \\
\hline M1 & $\mathrm{SNP}_{-} \mathrm{MS} 487-2$ & .4316 & .9652 \\
\hline M2 & SNP_ MS 478B14-848 & .6116 & 1.0 \\
\hline M3 & SNP_ MS 420M9-1395 & .7165 & .8258 \\
\hline M4 & SNP_D8S1810 & .3564 & .3418 \\
\hline
\end{tabular}

C-A Trend: Cochran-Armitage trend test was used for analysis of SNPs and 'pseudoSNPs';

K-S: Kolmogórov-Smirnov non-parametric test was applied to the whole range of genotypes of microsatellites (not using the 'pseudoSNP' approach). 


\section{Table S6.}

Association of age of onset and PANSS positive score with NRG1 genetic variability. Patients with an early onset of the disease ( $\leq 20$ years) were compared to the remaining data set. Likewise, patients with the severest positive psychopathology (PANSS positive score $>18$ ) were compared to the other patients. Results are shown for the global genotypic comparison or by grouping according to the homozygous carriers of the protective variants against the others.

\begin{tabular}{|c|c|c|c|c|c|c|c|c|c|}
\hline & \multirow{3}{*}{ Marker } & \multicolumn{4}{|c|}{ p-Value genotypic comparison } & \multicolumn{4}{|c|}{$\begin{array}{c}\text { p-Value protective genotype versus all } \\
\text { other genotypes }\end{array}$} \\
\hline & & \multicolumn{2}{|c|}{$\begin{array}{c}\text { Age of onset } \\
\text { test }\end{array}$} & \multicolumn{2}{|c|}{$\begin{array}{l}\text { PANSS positive } \\
\text { test }\end{array}$} & \multicolumn{2}{|c|}{$\begin{array}{c}\text { Age of onset } \\
\text { test }\end{array}$} & \multicolumn{2}{|c|}{$\begin{array}{l}\text { PANSS positive } \\
\text { test }\end{array}$} \\
\hline & & JT & Wilcoxon & $J T$ & Wilcoxon & JT & Wilcoxon & JT & Wilcoxon \\
\hline S1 & SNP8NRG221132 & 1581 & & .9952 & & .0739 & & .4991 & \\
\hline S2 & SNP8NRG221533 & .0015 & & .4722 & & $<.0001$ & & .1490 & \\
\hline s3 & SNP8NRG241930 & .2155 & & .2140 & & 1319 & & .3811 & \\
\hline S4 & SNP8NRG243177 & .0589 & & .1644 & & .0155 & & .0215 & \\
\hline M1 & SNP_MS 487-2 & .4682 & 0537 & .9265 & .2462 & 2503 & .4765 & .4823 & .4733 \\
\hline M2 & SNP_ MS 478B14-848 & .4102 & .0651 & .7613 & .8979 & .3862 & .1425 & .2678 & .3412 \\
\hline M3 & SNP_ MS 420M9-1395 & .4376 & .5713 & .6518 & .7118 & .0835 & .0233 & .2426 & 1759 \\
\hline M4 & SNP_D8S1810 & .2182 & .1566 & .2214 & 1982 & .0811 & .0297 & .4268 & 3139 \\
\hline
\end{tabular}

JT: Jonckheere-Terpstra trend-test for categorized values was used for the analysis of SNPs and 'pseudoSNPs'

Wilcoxon: Wilcoxon rank sum test was applied to the whole range of genotypes of microsatellite markers (not using the 'pseudoSNP' approach) 
Table S7a.

Genotype distribution in patients grouped by age of onset

\begin{tabular}{|c|c|c|c|c|c|c|c|c|}
\hline Age of onset & \multicolumn{2}{|c|}{$\leq 20$} & \multicolumn{2}{|c|}{$21-30$} & \multicolumn{2}{|c|}{$31-50$} & \multicolumn{2}{|c|}{$>50$} \\
\hline \multicolumn{9}{|l|}{ SNP8NRG221132 (S1) } \\
\hline GG & 202 & $78 \%$ & 343 & $76 \%$ & 164 & $67 \%$ & 18 & $90 \%$ \\
\hline$A G$ & 52 & $20 \%$ & 97 & $22 \%$ & 78 & $32 \%$ & 2 & $10 \%$ \\
\hline$A A$ & 4 & $2 \%$ & 9 & $2 \%$ & 1 & $<1 \%$ & - & $0 \%$ \\
\hline \multicolumn{9}{|l|}{ SNP8NRG221533 (S2) } \\
\hline$T T$ & 88 & $34 \%$ & 214 & $48 \%$ & 120 & $49 \%$ & 9 & $45 \%$ \\
\hline$C T$ & 142 & $55 \%$ & 188 & $42 \%$ & 89 & $37 \%$ & 10 & $50 \%$ \\
\hline CC & 28 & $11 \%$ & 47 & $10 \%$ & 34 & $14 \%$ & 1 & $5 \%$ \\
\hline \multicolumn{9}{|l|}{ SNP8NRG241930 (S3) } \\
\hline GG & 113 & $44 \%$ & 185 & $41 \%$ & 89 & $37 \%$ & 9 & $45 \%$ \\
\hline$T G$ & 116 & $45 \%$ & 206 & $46 \%$ & 117 & $48 \%$ & 10 & $50 \%$ \\
\hline$T T$ & 29 & $11 \%$ & 57 & $13 \%$ & 37 & $15 \%$ & 1 & $5 \%$ \\
\hline \multicolumn{9}{|l|}{ SNP8NRG243177 (S4) } \\
\hline$C C$ & 87 & $34 \%$ & 186 & $42 \%$ & 98 & $40 \%$ & 9 & $45 \%$ \\
\hline$C T$ & 132 & $51 \%$ & 196 & $44 \%$ & 111 & $46 \%$ & 9 & $45 \%$ \\
\hline$T T$ & 39 & $15 \%$ & 64 & $14 \%$ & 33 & $14 \%$ & 2 & $10 \%$ \\
\hline \multicolumn{9}{|l|}{ SNP_MS 487-2 (M1) } \\
\hline Short/Short & 87 & $34 \%$ & 167 & $37 \%$ & 85 & $35 \%$ & 6 & $30 \%$ \\
\hline Long/Short & 128 & $50 \%$ & 218 & $49 \%$ & 118 & $49 \%$ & 11 & $55 \%$ \\
\hline Long/Long & 41 & $16 \%$ & 63 & $14 \%$ & 39 & $16 \%$ & 3 & $15 \%$ \\
\hline \multicolumn{9}{|c|}{ SNP_MS 478B14-848 (M2) } \\
\hline Short/Short & 61 & $24 \%$ & 120 & $27 \%$ & 69 & $29 \%$ & 5 & $25 \%$ \\
\hline Long/Short & 127 & $50 \%$ & 222 & $50 \%$ & 106 & $44 \%$ & 8 & $40 \%$ \\
\hline Long/Long & 67 & $26 \%$ & 106 & $24 \%$ & 67 & $28 \%$ & 7 & $35 \%$ \\
\hline \multicolumn{9}{|c|}{ SNP_MS 420M9-1395 (M3) } \\
\hline Short/Short & 135 & $53 \%$ & 234 & $52 \%$ & 121 & $50 \%$ & 8 & $40 \%$ \\
\hline Long/Short & 105 & $41 \%$ & 180 & $40 \%$ & 95 & $40 \%$ & 10 & $50 \%$ \\
\hline Long/Long & 15 & $6 \%$ & 35 & $8 \%$ & 24 & $10 \%$ & 2 & $10 \%$ \\
\hline \multicolumn{9}{|l|}{ SNP_D8S1810 (M4) } \\
\hline Short/Short & 15 & $6 \%$ & 35 & $8 \%$ & 24 & $10 \%$ & 2 & $11 \%$ \\
\hline Long/Short & 87 & $35 \%$ & 155 & $36 \%$ & 86 & $37 \%$ & 6 & $32 \%$ \\
\hline Long/Long & 146 & $59 \%$ & 245 & $56 \%$ & 123 & $53 \%$ & 11 & $58 \%$ \\
\hline
\end{tabular}


Table S7b.

Genotype distribution in patients grouped by PANSS positive score

\begin{tabular}{|c|c|c|c|c|c|c|c|c|}
\hline PANSS positive score & \multicolumn{2}{|c|}{ I: 7-8 } & \multicolumn{2}{|c|}{ II: $9-12$} & \multicolumn{2}{|c|}{ III: 13-17 } & \multicolumn{2}{|c|}{ IV: 18-38 } \\
\hline$G G$ & 189 & $73 \%$ & 209 & $75 \%$ & 188 & $77 \%$ & 173 & $75 \%$ \\
\hline$A G$ & 65 & $25 \%$ & 64 & $23 \%$ & 53 & $22 \%$ & 54 & $23 \%$ \\
\hline$A A$ & 4 & $2 \%$ & 5 & $2 \%$ & 2 & $1 \%$ & 3 & $1 \%$ \\
\hline \multicolumn{9}{|l|}{ SNP8NRG221533 (S2) } \\
\hline$T T$ & 119 & $46 \%$ & 130 & $47 \%$ & 103 & $42 \%$ & 95 & $41 \%$ \\
\hline CT & 112 & $43 \%$ & 118 & $42 \%$ & 104 & $43 \%$ & 110 & $48 \%$ \\
\hline$C C$ & 27 & $10 \%$ & 30 & $11 \%$ & 36 & $15 \%$ & 25 & $11 \%$ \\
\hline \multicolumn{9}{|l|}{ SNP8NRG241930 (S3) } \\
\hline GG & 96 & $37 \%$ & 121 & $44 \%$ & 102 & $42 \%$ & 97 & $42 \%$ \\
\hline$T G$ & 129 & $50 \%$ & 113 & $41 \%$ & 103 & $42 \%$ & 115 & $50 \%$ \\
\hline$T T$ & 33 & $13 \%$ & 42 & $15 \%$ & 38 & $16 \%$ & 18 & $8 \%$ \\
\hline \multicolumn{9}{|l|}{ SNP8NRG243177 (S4) } \\
\hline$C C$ & 111 & $43 \%$ & 108 & $39 \%$ & 98 & $41 \%$ & 77 & $33 \%$ \\
\hline CT & 112 & $44 \%$ & 126 & $45 \%$ & 105 & $44 \%$ & 122 & $53 \%$ \\
\hline$T T$ & 34 & $13 \%$ & 43 & $16 \%$ & 38 & $16 \%$ & 31 & $13 \%$ \\
\hline \multicolumn{9}{|l|}{ SNP_MS 487-2 (M1) } \\
\hline Short/Short & 91 & $36 \%$ & 94 & $34 \%$ & 86 & $36 \%$ & 81 & $35 \%$ \\
\hline Long/Short & 118 & $46 \%$ & 145 & $53 \%$ & 122 & $51 \%$ & 115 & $50 \%$ \\
\hline Long/Long & 47 & $18 \%$ & 37 & $13 \%$ & 33 & $14 \%$ & 34 & $15 \%$ \\
\hline \multicolumn{9}{|l|}{ SNP_MS 478B14-848 (M2) } \\
\hline Short/Short & 61 & $24 \%$ & 65 & $24 \%$ & 60 & $25 \%$ & 64 & $28 \%$ \\
\hline Long/Short & 126 & $49 \%$ & 142 & $51 \%$ & 119 & $49 \%$ & 102 & $44 \%$ \\
\hline Long/Long & 68 & $27 \%$ & 69 & $25 \%$ & 62 & $26 \%$ & 64 & $28 \%$ \\
\hline \multicolumn{9}{|l|}{ SNP_MS 420M9-1395 (M3) } \\
\hline Short/Short & 131 & $52 \%$ & 144 & $52 \%$ & 124 & $51 \%$ & 121 & $53 \%$ \\
\hline Long/Short & 101 & $40 \%$ & 104 & $38 \%$ & 103 & $43 \%$ & 92 & $40 \%$ \\
\hline Long/Long & 22 & $9 \%$ & 29 & $10 \%$ & 14 & $6 \%$ & 16 & $7 \%$ \\
\hline \multicolumn{9}{|l|}{ SNP_D8S1810 (M4) } \\
\hline Short/Short & 19 & $8 \%$ & 25 & $9 \%$ & 17 & $7 \%$ & 19 & $9 \%$ \\
\hline Long/Short & 90 & $37 \%$ & 94 & $35 \%$ & 98 & $42 \%$ & 71 & $32 \%$ \\
\hline Long/Long & 137 & $56 \%$ & 150 & $56 \%$ & 120 & $51 \%$ & 133 & $60 \%$ \\
\hline
\end{tabular}




\section{Table S8.}

Association study of disease-related (basic cognition/fine motor function as measured by the tapping test) and disease-unrelated (body length) control variables with NRG1 genotypes. Results are shown after grouping of subjects into quartiles according to the respective variables. P-Values refer to the comparison of carriers of the protective variants against the others.

\begin{tabular}{|c|c|c|c|c|c|}
\hline & \multirow{3}{*}{ Marker } & \multicolumn{4}{|c|}{$\begin{array}{c}\text { p-Value protective genotype versus } \\
\text { other genotypes }\end{array}$} \\
\hline & & \multicolumn{2}{|c|}{$\begin{array}{c}\text { Tapping } \\
\text { test }\end{array}$} & \multicolumn{2}{|c|}{$\begin{array}{c}\text { Body length } \\
\text { test }\end{array}$} \\
\hline & & JT & Wilcoxon & JT & Wilcoxon \\
\hline S1 & SNP8NRG221132 & .3752 & & .1829 & \\
\hline S2 & SNP8NRG221533 & .1066 & & .3933 & \\
\hline S3 & SNP8NRG241930 & .3858 & & .4130 & \\
\hline S4 & SNP8NRG243177 & .1378 & & .4963 & \\
\hline M1 & SNP_MS 487-2 & .3498 & .3499 & .2957 & .2958 \\
\hline M2 & $\mathrm{SNP}_{-}$MS 478B14-848 & .2338 & .2338 & .3461 & .3461 \\
\hline M3 & $\mathrm{SNP}_{-} \mathrm{MS} 420 \mathrm{M9}-1395$ & .1953 & .1953 & .1704 & .1705 \\
\hline M4 & SNP_D8S1810 & .2808 & .2809 & .3560 & .3561 \\
\hline
\end{tabular}

JT: Jonckheere-Terpstra trend-test for categorized values was used for analysis of SNPS and 'pseudoSNPS'

Wilcoxon: Wilcoxon rank sum test was applied to the whole range of genotypes of microsatellites (not using the 'pseudoSNP' approach) 


\section{Supplementary Figure}

\section{Figure S1.}

Summary of statistically significant protective haplotypes observed in the haplotypic analysis of the NRG1 region of interest with UNPHASED (blue rectangles). All p-values shown are nominal. \%Patients and \%Controls refer to the frequency of each haplotypic combination in GRAS patients and controls, respectively. Odds ratio (OR) and 95\% Cornfield confidence interval $(95 \% \mathrm{cl})$ for each protective haplotypic combination is also shown. Please note the atrisk-haplotype region described in the Icelandic population (Stefansson et al., 2002) at the bottom (yellow-filled rectangle) as well as the red-framed upright rectangles focusing on those markers where opposite alleles exert opposite effects (protection/risk).

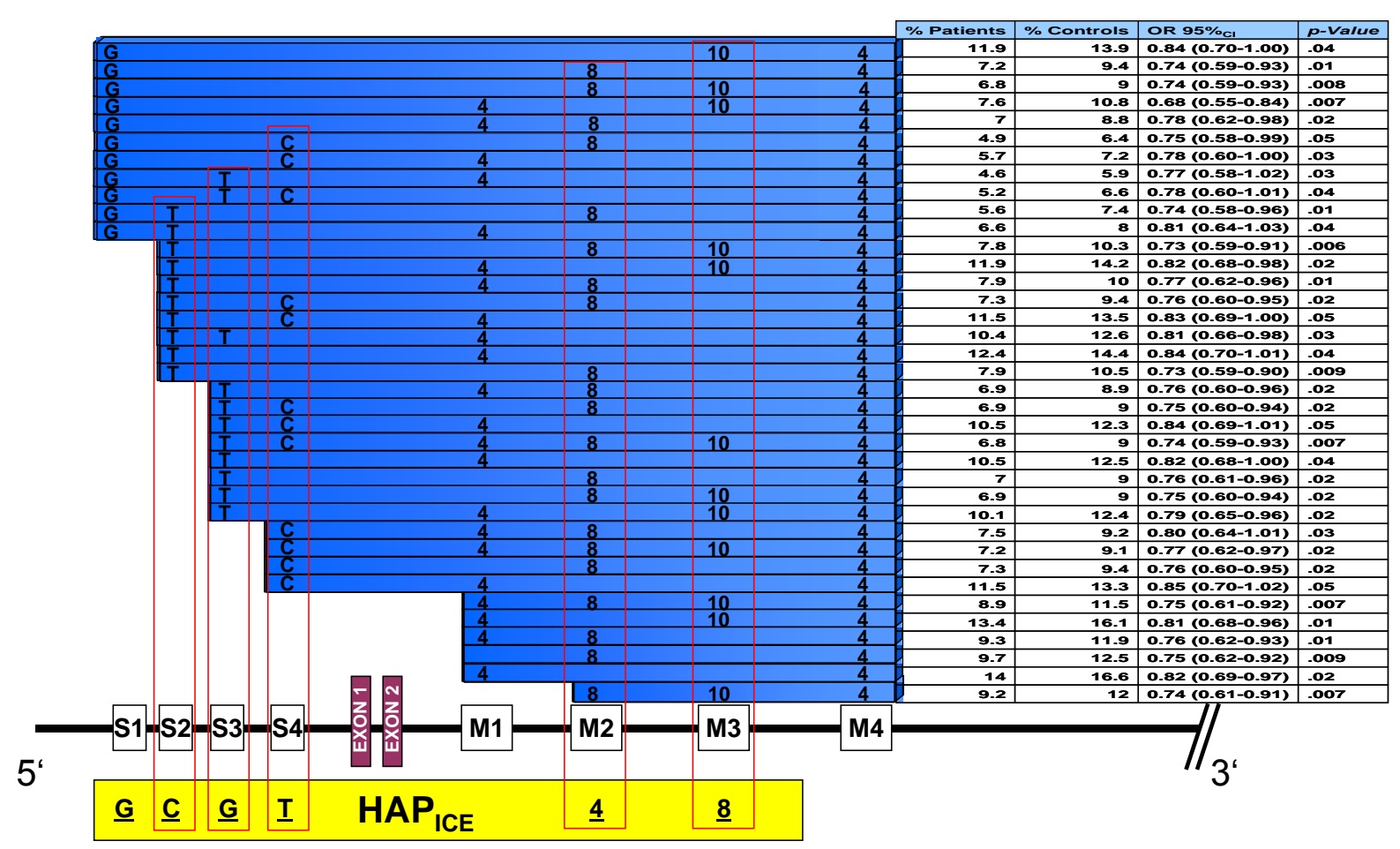

EXON1: NRG1 Type IV-specific exon

EXON2: NRG1 Type II-specific exon

S1: SNP8NRG221132

S2: SNP8NRG221533

S3: SNP8NRG241930

S4: SNP8NRG243177

M1: MS 487-2

M2: MS 478B14-848

M3: MS 420M9-1395

M4: D8S1810 


\section{Supplementary Methods}

\section{Genetic Analysis}

\section{DNA Extraction and Normalization}

Genomic DNA was purified from whole blood using JETQUICK Blood \& Cell Culture DNA Spin Kit (Genomed GmbH, Löhne, Germany) according to the manufacturer's protocol. Resulting DNA samples were aliquoted and stored at $-80^{\circ} \mathrm{C}$. For further analysis, DNA was normalized to $50 \mathrm{ng} / \mu \mathrm{l}$ with an automated robotic platform (Microlab Star, Hamilton, Bonaduz, Switzerland). For quality control, each sample was analyzed with a $0.8 \%$ agarose gel.

\section{Single Nucleotide Polymorphism Analysis}

The SNPs (S2: SNP8NRG221533 \& S3: SNP8NRG241930) were analyzed using Simple Probes (TIB Molbiol, Berlin, Germany) and called using the LightCycler 840 Genotyping Software implemented in the LightCycler® 480 system (Roche, Mannheim, Germany). The reaction mixture $(10 \mu \mathrm{l})$ was prepared with $40 \mathrm{ng}$ of DNA in 384 well plates according to standard protocols (Roche). The concentration of $\mathrm{MgCl}_{2}$ and the Genotyping Master Mix were adapted for each assay. The cycle conditions were as follows: denaturation of the template DNA with $95^{\circ} \mathrm{C}$ for $10 \mathrm{~min}$, amplification of the target DNA for 45 cycles of (1) $95^{\circ} \mathrm{C}$ for $10 \mathrm{~s}$, (2) $60^{\circ} \mathrm{C}$ for $10 \mathrm{~s}$ and (3) $72^{\circ} \mathrm{C}$ for $15 \mathrm{~s}$ (temperature ramp rate $4.6^{\circ} \mathrm{C} / \mathrm{s}$ in steps (1) and (3) and $2.4^{\circ} \mathrm{C}$ in (2)). Melting curve analysis was performed with $30 \mathrm{~s}$ of $95^{\circ} \mathrm{C}, 40^{\circ} \mathrm{C}$ for $2 \mathrm{~min}$ (ramp rate $2.0^{\circ} \mathrm{C} / \mathrm{s}$ ) and then a continuous ramping to $75^{\circ} \mathrm{C}$ with 3 acquisitions per ${ }^{\circ} \mathrm{C}$. In each run, 8 positive controls (hgDNA, Bioline, Luckenwalde, Germany) and negative water blanks were included for quality and internal control purposes.

The SNPs (1: SNP8NRG221132 \& S4: SNP8NRG243177) were analyzed using HybProbes (TIB Molbiol, Berlin, Germany) in the same LightCycler® 480 system (Roche). The reaction mixture $(5 \mu \mathrm{l})$ was prepared with $40 \mathrm{ng}$ of DNA in 384 well plates according to standard protocols (Roche). Genotyping Master Mix was used in a $0.5 X$ concentration.

\section{S1: SNP8NRG221132}

ARIA F: 5' CTAATTTCTCCTGAACTCTGTATAAC 3'

ARIA R: 5' GAGGCAGTAACAAAATAGACC 3'

Sensor mut: 5' ACATTACTACCTTACATTGATATATGCATG-FL 3' 
Anchor: 5' 640-CTTATACATTGTACATTCTGCTTAC p 3'

\section{S4: SNP8NRG243177}

NRG1 F: 5' CAAAACAAGGTTCCATTCTCTCAAA 3'

NRG1 R: 5' CAGTCCAAATTGCCAACTTGC 3'

Sensor A: 5' CCAGTATACATTCACTTGAACCCT-FL 3'

Anchor: 5' 640-CATGGTGCTTCTAGCGATTTACTGAAA p 3'

Cycling conditions: denaturation of the template DNA with $95^{\circ} \mathrm{C}$ for $10 \mathrm{~min}$, amplification of the target DNA for 45 cycles of (1) $95^{\circ} \mathrm{C}$ for $10 \mathrm{~s}$, (2) $45^{\circ} \mathrm{C}$ (S1) or $52^{\circ} \mathrm{C}$ (S4) for $15 \mathrm{~s}$ and $(3) 72^{\circ} \mathrm{C}$ for $10 \mathrm{~s}$ (S1) or $18 \mathrm{~s}$ (S4) (temperature ramp rate $4.8^{\circ} \mathrm{C} / \mathrm{s}$ in steps (1) and (3) and $2.5^{\circ} \mathrm{C}$ in (2)). Melting curve analysis was performed with $1 \mathrm{~min}$ of $95^{\circ} \mathrm{C}, 40^{\circ} \mathrm{C}$ for $2 \mathrm{~min}$ (ramp rate $2.0^{\circ} \mathrm{C} / \mathrm{s}$ ) and then a continuous ramping to $75^{\circ} \mathrm{C}$ with 5 acquisitions per ${ }^{\circ} \mathrm{C}$. In each run, 8 positive controls (hgDNA, Bioline) and negative water blanks were included for quality and internal control purposes.

\section{Microsatellite Analysis}

Four polymorphic repeats in Intron 2 of NRG1 were amplified from genomic DNA by PCR. Primers for tetranucleotide repeat 487-2 and dinucleotide repeats 478B14-848 and 420M9-1395 were chosen according to Steffanson et al. 2002 (Stefansson et al., 2002) (deCODE Genetics http://www.decode.com/nrg1/markers/). Alleles "0" of microsatellites $478 \mathrm{~B} 14-848$ and $420 \mathrm{M} 9-1395$ as described in (Stefansson et al., 2002) correspond to alleles " 4 " and " 8 ", respectively, in our data analysis.

\section{M1: MS 487-2 (tetranucleotide repeat)}

Forward: 5' AGTGAGTAGGGCTGGCTGCT 3' (VIC-labeled)

Reverse: 5' GCTGCTAATATGGCCCCTTC 3'

M2: MS 478B14-848 (dinucleotide repeat)

Forward: 5' CCACATGTCCAACTGAAGAGG 3' (FAM-labeled)

Reverse: 5' TCTCCATGTGTAAAACAATACATATCA 3'

M3: MS 420M9-1395 (dinucleotide repeat)

Forward:: 5' CTTTTAATCATGAAAGAATAGCAAAAA 3' (FAM-labeled)

Reverse: 5' TGTTGTTGTATATTTCAGAATTTCCTT 3' 
Primers for dinucleotide repeat DS1810 (Accession number: GDB:613185) were chosen according to the sequences described in GenBank, (http://www.ncbi.n/m.nih.gov/Genbank/)):

\section{M4: D8S1810 (dinucleotide repeat)}

Forward: 5' ATGATGCTGAGTCCCCA 3' (FAM-labeled)

Reverse: 5' CAGAGGGCTGATTTTATGC 3'

For each sample, the reaction mixture $(20 \mu \mathrm{L})$ was prepared in 384 well plates, each

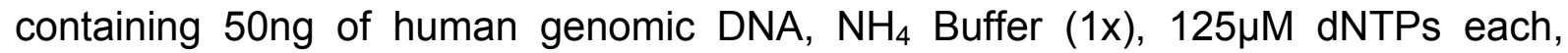
$2.5 \mathrm{mM} \mathrm{MgCl}_{2}$, 200nM FAM-labeled forward and reverse primers, and $1 \mathrm{U}$ Diamond polymerase (Bioline). The cycling program was carried out after a preheating step at $94^{\circ} \mathrm{C}$ for $5 \mathrm{~min}$ and included 30 cycles of (1) denaturation at $94^{\circ} \mathrm{C}$ for $30 \mathrm{~s}$, (2) annealing at $65^{\circ} \mathrm{C}$ for 30 s and (3) extension at $72^{\circ} \mathrm{C}$ for $60 \mathrm{~s}$ in a DNA Thermal Cycler (PTC-200 MJ Research, BioRad, Munich, Germany).

The amplicons were separated using size electrophoresis on the ABI 3730 XL DNA Analyzer. Samples were diluted 1:50 with $0.3 \mathrm{mM}$ EDTA and $4 \mu \mathrm{l}$ were mixed with $6 \mu \mathrm{l}$ LIZ-500 Size Standard (Applied Biosystems, Foster City, USA). Raw data were processed using the Gene Mapper Software 4.0 (Applied Biosystems).

Overall, successfully genotyped markers amounted to $97.2-99.1 \%$.

\section{Supplementary Material - References}

Stefansson H, Sigurdsson E, Steinthorsdottir V, Bjornsdottir S, Sigmundsson T, Ghosh S, Brynjolfsson J, Gunnarsdottir S, Ivarsson O, Chou TT et al. . 2002. Neuregulin 1 and susceptibility to schizophrenia. Am J Hum Genet 71(4):87792. 